\title{
Bayesian persuasion
}

Citation for published version (APA):

Kerman, T. T. (2021). Bayesian persuasion: Elections and informativeness of beliefs. [Doctoral Thesis, Maastricht University]. Maastricht University. https://doi.org/10.26481/dis.20210621tk

Document status and date:

Published: 01/01/2021

DOI:

10.26481/dis.20210621tk

Document Version:

Publisher's PDF, also known as Version of record

\section{Please check the document version of this publication:}

- A submitted manuscript is the version of the article upon submission and before peer-review. There can be important differences between the submitted version and the official published version of record.

People interested in the research are advised to contact the author for the final version of the publication, or visit the DOI to the publisher's website.

- The final author version and the galley proof are versions of the publication after peer review.

- The final published version features the final layout of the paper including the volume, issue and page numbers.

Link to publication

\footnotetext{
General rights rights.

- You may freely distribute the URL identifying the publication in the public portal. please follow below link for the End User Agreement:

www.umlib.nl/taverne-license

Take down policy

If you believe that this document breaches copyright please contact us at:

repository@maastrichtuniversity.nl

providing details and we will investigate your claim.
}

Copyright and moral rights for the publications made accessible in the public portal are retained by the authors and/or other copyright owners and it is a condition of accessing publications that users recognise and abide by the legal requirements associated with these

- Users may download and print one copy of any publication from the public portal for the purpose of private study or research.

- You may not further distribute the material or use it for any profit-making activity or commercial gain

If the publication is distributed under the terms of Article $25 \mathrm{fa}$ of the Dutch Copyright Act, indicated by the "Taverne" license above, 


\title{
Bayesian Persuasion: Elections and Informativeness of Beliefs
}

\author{
Toygar T. Kerman
}

2021

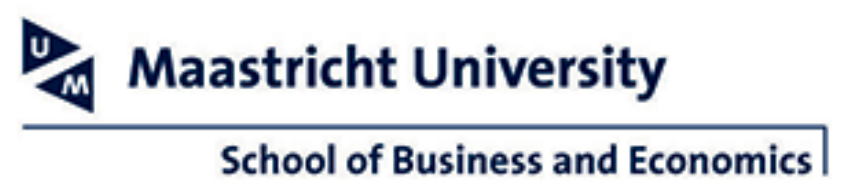


(C) Toygar T. Kerman, Maastricht 2021

All rights reserved. No part of this publication may be reproduced, stored in an automated data system, or transmitted in any form or by any means, electronic, mechanical, photocopying, recording or otherwise, without prior permission of the author.

This book was typeset by the author using $\mathrm{AT}_{\mathrm{E} X} \mathrm{X}$. The cover art is the work of Nazlı Tola and Firat Doğu Gül. 


\title{
Bayesian Persuasion: Elections and Informativeness of Beliefs
}

\author{
Dissertation
}

To obtain the degree of Doctor at Maastricht University on the authority of the Rector Magnificus Prof. Dr. Rianne M. Letschert, in accordance with the decision of the Board of Deans, to be defended in public on on Monday June 21, 2021 at 13.00 hours. 
Supervisor:

Co Supervisor:
Prof. Dr. Jean-Jacques Herings

Prof. Dr. Dominik Karos (University of Bielefeld, Bielefeld)

Assessment Committee: Prof. Dr. Rudolf Müller (Chair)

Dr. Elias Tsakas

Prof. Dr. Friederike Mengel (University of Essex, Essex)

Prof. Dr. Berno Büchel (University of Fribourg, Fribourg) 

"Nothing is so unbelievable that oratory cannot make it acceptable."

- Cicero 



\title{
Acknowledgements
}

\author{
"For long you live and high you fly \\ And smiles you'll give and tears you'll cry \\ And all you touch and all you see \\ Is all your life will ever be"
}

- Pink Floyd, 1973, Breathe/Dark Side of the Moon

These amazing lyrics by Pink Floyd make a very accurate (and equally touching) summary of a person's life; one part of it is simply "all you touch and all you see". In this sense, and in any other sense for that matter, Maastricht was a very important episode in my life, with all its ups and downs. I am glad to have this opportunity to thank everyone who helped me throughout this turbulent journey.

First and foremost, I thank my advisors P. Jean-Jacques Herings and Dominik Karos for their immense support. It is needless to say that this journey of mine would come to an abrupt end without their guidance. Whenever I hit a roadblock or felt unsure about myself, they were there to pick the pieces up. I thank Jean-Jacques for all his guidance in seeing the big picture and showing me how to connect the (sometimes invisible) dots in research; I can only hope to become as insightful as him one day. I thank Dominik for always encouraging me, not just in research but in all aspects of academic life (I would most likely not meet even half of the seminar speakers that were invited to our university if it was not for him). There were many times I faced a lock that wouldn't open and in each of those times, he provided a lockpick. I will miss the countless hours I spent in his office trying to solve a problem, as well as the hours we spent in Jean-Jacques' office trying to improve the papers.

I thank my advisor at Bilkent University, Semih Koray, for all his guidance throughout my time at Bilkent and for introducing me to the beauties of economic theory. I thank one of my earlier mentors, Emin Karagözoğlu (who also did his PhD at Maastricht University), for directing me to Maastricht and opening the door for a great $\mathrm{PhD}$ experience. It is without a doubt that my path would not cross with Maastricht if I hadn't met him. I also want to thank all the other professors at Bilkent University who inspired me to further my studies in economics; Nuh Aygün Dalkıran, Refet Gürkaynak, Tarık Kara, Sang Seok Lee, Cavit Pakel, Çă̆rı Sağlam, and Kemal Yıldız. 
I thank my friend and coauthor Anastas P. Tenev for all the time we spent together, whether it was in conferences, working on a project, or simply having a conversation. Besides being a pleasure to work with, he was always available for blowing off some steam about the difficulties of various aspects of academic life. One thing that is certain is that coauthoring the last paper of our dissertations eased the last stage of my $\mathrm{PhD}$ immensely. I will even miss working on the project during Covid-19, in the seemingly abandoned building of our department.

I am glad to have shared an office (at least for the most part of my PhD) with Hyemi Jin, Lina Maria Lozano, and Eveline Vandewal, and I thank them for always providing a nice and calm environment to work in. Their presence made the (sometimes excruciating) experience of obtaining a PhD much easier. I will surely miss complaining about PhD life (and gossiping) with them. I thank Lina also for the great parties she had at her place (and the guacamole that came with them), those were always something to look forward to. I also thank my office neighbours Marcia Diaz Claudio and Jacqueline Kurz, it was great to know that my breaks from work would always be enjoyable. I thank Marcia also for all the fun times we spent together just drinking and chatting, for teaching me some card and board games that I didn't even know existed, and for introducing me to some vegan dishes that I would definitely not try on my own.

I thank everyone in the Microeconomics and Public Economics (a.k.a. AE1) department for providing a friendly and constructive environment to work in. I thank the professors whom I had the pleasure of being a tutor for their course; Christian Kerckhoffs, Karsten Mau, Mark Sanders, Stefan Terstiege, Elias Tsakas, Alexander Vostroknutov, Peter Werner, and Matthias Wibral. I have learned plenty about structuring courses, devising homeworks, and preparing exams from them. I also thank Andrew Mackenzie for his help during the job market, and Burak Can for his support and for the fun conversations we had during our smoke breaks. I thank Elke Lucas for always being available to help with virtually anything and for always having a smile. I thank Sylvia Beenen for being flexible with the chaotic scheduling issues each period and always trying to look for Pareto improvements. I would also like to thank the members of the assessment committee, Berno Büchel, Friederike Mengel, Rudolf Müller, and Elias Tsakas for taking the time to review this dissertation and for their helpful suggestions.

There were quite a few Turkish PhD students in Maastricht when I arrived who helped me adapt to life in Maastricht. I thank Emre Ergin and Kutay Cingiz for showing me the initial ropes and for the conversations we had. I also thank Hande Erkut, Seher Fazlıoğlu, Mehmet Ismail, and Merve Özer for their moral support.

I am thankful to all my fellow PhDs at Maastricht University who made this experience a much better one than it could've been. I thank Henrik Zaunbrecher for always being so fun to have a conversation with, as well as for rekindling my interest in Scotch by having those Scotch nights following brutal days of teaching. I additionally thank Aditya Aradhye, Péter 
Bayer, Nickolas Gagnon, Laura Kasper, Jasmine Maes, Niels Mourmans, Marcello Negrini, Riccardo Saulle, Frauke Stehr, and Niloofar Yousefimanesh for all the memories we shared at parties, conferences, and drinks. I also thank my long-time friend Firat Doğu Gül and Nazlı Tola or their help in the process of designing the cover art for the dissertation.

As they say, "last but not the least", I thank my family for not just their endless support during my PhD, but throughout my whole life. I thank my parents for always encouraging me and believing in me, even at times when I didn't believe in myself. I don't think it is possible to put in words how much of a comfort was to know that they were always there. Neither can I find the words that do justice to my gratitude for my spouse, Gülşen; I thank her for her support through both my best and worst of times. She was always there to share my joy and my sorrow, I am indebted to her for her help during this episode and for everything prior to it. I am thankful to our cats Piki and Periş for (knowingly or unknowingly) contributing to my mental wellbeing during this time. Finally, I am thankful to our dog back in Turkey, Tarçın (who passed away while this dissertation was being written), for always providing me much needed moral support even from afar, and for all the beautiful memories we shared. 


\section{Contents}

$\begin{array}{ll}\text { Acknowledgements } & \text { ix }\end{array}$

1 Introduction $\quad 1$

2 Bayesian Persuasion in Elections with Sincere Voting $\quad 7$

2.1 Introduction . . . . . . . . . . . . . . . . . . . . . . . 8

2.1.1 Overview of Results . . . . . . . . . . . . . . . . . . . 9 9

2.1.2 An Illustrative Example . . . . . . . . . . . . . . . . . . 10

2.1.3 Related Literature . . . . . . . . . . . . . . . . . . . . . . . 12

2.2 Preliminaries and Notation . . . . . . . . . . . . . . . . . . . . 14

2.2.1 Communication Strategies and Beliefs . . . . . . . . . . . . . . . 14

2.2.2 Voting Problems . . . . . . . . . . . . . . . . . . . 15

2.2.3 Persuasion . . . . . . . . . . . . . . . . . . 16

2.3 Optimal Communication . . . . . . . . . . . . . . . . 17

2.3.1 Straightforward Communication Strategies . . . . . . . . . . . . . 17

2.3.2 Optimal Public Communication . . . . . . . . . . . . . . . . . . . 18

2.3.3 Optimal Private Communication . . . . . . . . . . . . . . . . . 19

2.4 Swing Voter's Curse . . . . . . . . . . . . . . . . . . 22

2.5 Communication with Equilibrium Constraints . . . . . . . . . . . . 25

2.6 Conclusion . . . . . . . . . . . . . . . . . . . . . . . . . 29

2.7 Appendix . . . . . . . . . . . . . . . . . . . . . . . . . . . . . . 29

2.7.1 Straightforwardness . . . . . . . . . . . . . . . . 29

2.7 .2 Proofs . . . . . . . . . . . . . . . . . . . 31

3 Bayesian Persuasion in Communication Networks 38

3.1 Introduction . . . . . . . . . . . . . . . . . . . . . . 39

3.1 .1 An Illustrative Example . . . . . . . . . . . . . . . . . . 39

3.1 .2 Overview of Results . . . . . . . . . . . . . . . . . 41

3.1.3 Related Literature . . . . . . . . . . . . . . . . . . . . . . . . 42

3.2 Notation . . . . . . . . . . . . . . . . . . . . . 44 
3.2.1 Communication Strategy . . . . . . . . . . . . . . . . . 44

3.2.2 Networks . . . . . . . . . . . . . . . . . . . . . . 45

3.2 .3 Voting . . . . . . . . . . . . . . . . . . 46

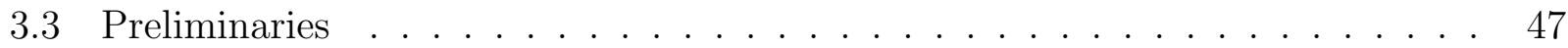

3.3.1 Optimal Public Communication on the Empty Network . . . . . . . . . 47

3.3.2 The Effects of the Network . . . . . . . . . . . . . . . . . . . . . . 48

3.3.3 Straightforwardness . . . . . . . . . . . . . . . . . . 50

3.3.4 Truth-Telling in the Sender's Preferred State . . . . . . . . . . . . . 52

3.4 General Observations . . . . . . . . . . . . . . . . . . . . . 53

3.4.1 The Network Does not Benefit the Sender . . . . . . . . . . . . . . 53

3.4 .2 Networks with Isolated Nodes . . . . . . . . . . . . . . . . . . . . 54

3.4.3 Networks Consisting of Complete Components . . . . . . . . . . . . . . 55

3.5 Optimality in Specific Network Structures . . . . . . . . . . . . . . . . . 57

3.5.1 Star Networks . . . . . . . . . . . . . . . . . . 57

3.5.2 Regular Networks . . . . . . . . . . . . . . . . . . . 58

3.5.3 Irregular Networks . . . . . . . . . . . . . . . . . . . . . . . . 61

3.6 Adding Links . . . . . . . . . . . . . . . . . . . . . . . . 63

3.7 Discussion and Conclusion . . . . . . . . . . . . . . . . 65

3.8 Appendix . . . . . . . . . . . . . . . . . . . . . . . 67

$\begin{array}{lll}4 & \text { Belief Inducibility and Informativeness } & 77\end{array}$

4.1 Introduction . . . . . . . . . . . . . . . . . . . . . . 78

4.2 Related Literature . . . . . . . . . . . . . . . . . . . . . . . 81

4.3 Preliminaries and Notation . . . . . . . . . . . . . . . . . . . . . 82

4.4 Implementing Belief Vectors . . . . . . . . . . . . . . . . . . . . . . 84

4.5 Minimal and Direct Communication Strategies . . . . . . . . . . . . . . 85

4.6 Language Independent Communication Strategies . . . . . . . . . . . . . . 87

4.7 Inducible Distributions . . . . . . . . . . . . . . . . . . . . . . . . . . . . 90

4.8 The Information and Posterior Correspondences . . . . . . . . . . . . . . . . . 93

4.9 Informativeness of Communication Strategies . . . . . . . . . . . . . . . . 98

4.10 Conclusion . . . . . . . . . . . . . . . . . . . . . . . . 101

4.11 Appendix . . . . . . . . . . . . . . . . . . . 101

$\begin{array}{ll}\text { Bibliography } & 108\end{array}$

$\begin{array}{ll}\text { General Discussion } & 115\end{array}$

$\begin{array}{ll}\text { Summary } & 119\end{array}$ 
Impact

122

Biography

126 


\section{Chapter 1}

\section{Introduction}

"Persuasion is clearly a sort of demonstration, since we are most fully persuaded when we consider a thing to have been demonstrated."

- Aristotle

Persuasion can be defined as the act of changing someone's belief about a subject by sharing with them information they do not possess. Following Aristotle's interpretation of persuasion, sharing information is then a sort of demonstration and it could take many forms; providing statistics to your opponent to win an argument if you are a debater, supplying evidence to a judge to convict a defendant if you are a prosecutor, or even threatening someone by show of force to make them pay their debts if you are a mob boss. Consequently, since one of the most important factors that influence behavior is information, specifying information allocation is an important area of research in economics, which is called information design.

Before information design, a similar theory emerged in the 60 's that was first devised by Leonid Hurwicz. In this theory named mechanism design, one tries to design economic mechanisms (or incentives) to reach a desired outcome in a setting where agents act strategically and rationally. ${ }^{1}$ Here, the designer takes the information of agents as given and tries to design a mechanism to implement an outcome. One of the well known examples to mechanism design is an auction, where a seller is trying to sell a good to multiple buyers. In reality, we observe that the seller's choice of mechanism is usually the English auction, where the highest bidder gets the good and pays their bid as the price.

In contrast to mechanism design, an information designer takes the mechanism as given and allocates information among agents in order to implement an outcome. ${ }^{2}$ In other words, given a mechanism, the information designer provides (some) information to the agent(s)

\footnotetext{
${ }^{1}$ The 2007 Nobel Memorial Prize in Economic Sciences was awarded to Leonid Hurwicz, Eric Maskin, and Roger Myerson "for having laid the foundations of mechanism design theory".

${ }^{2}$ Bergemann and Morris (2016b) and Taneva (2019) provide a comparison of mechanism design and information design.
} 
with the goal of persuading them to take a certain action. Agents react to the information they receive in a rational way, that is, by updating their beliefs via Bayes rule. Hence, this process is called Bayesian persuasion, which is also the title of the seminal paper Kamenica and Gentzkow (2011). ${ }^{3}$

While Bayesian persuasion is similar to other communication models (for instance with cheap talk (Crawford and Sobel, 1982)), one major difference is the commitment assumption. That is, the sender chooses a communication protocol (a distribution of messages) that provides a realization, where the sender truthfully reports the realization to the receiver(s). The commitment assumption is usually in line with real-world problems; for instance, a drug company seeking approval of the FDA is required to report the results of the tests truthfully. However, the company might try to "cheat" in the tests and report a false outcome to the FDA. It is also possible to analyze these types of situations where there is no commitment or partial commitment by the sender; Best and Quigley (2016), Min (2017), Fréchette et al. (2019), and Lipnowski et al. (2019) all consider weakening of the commitment assumption in various ways. ${ }^{4}$

To illustrate how Bayesian persuasion works, let us consider a simple example in the spirit of Kamenica and Gentzkow (2011). ${ }^{5}$ Suppose that two people, Alex and Bob, want to go to a classical music concert. The final decision will be made by Bob, since it's his birthday. There are two concerts available: one of music of Bach and the other of Stravinsky. However, only one of the orchestras that are going to perform is high quality. While Alex wants to go to a Bach concert and does not care about the quality of the orchestra, Bob wants to go to a concert where the orchestra is high quality. Alex and Bob both have a prior belief $\operatorname{Pr}($ high $)=1 / 3$ that the orchestra performing Bach is high quality.

Suppose that Alex is not the best of friends and is trying to persuade Bob to go to a Bach concert. Bob agrees to go to a Bach concert if he believes with at least $50 \%$ probability that the orchestra is high quality. Alex tells Bob that he will do some research on the orchestra that will perform Bach. The choice of research, which is observed by Bob, could be thought of as involving decisions on which websites to check for information, which performances of the two orchestras to compare, and so on. This research can be formalized by distributions $\pi(\cdot \mid h i g h)$ and $\pi(\cdot \mid l o w)$ on some set of realizations, where high and low represent the two states of the world in which the orchestra is high and low quality, respectively. We call the distribution $\pi$

\footnotetext{
${ }^{3}$ Following one of the founders of information design, Emir Kamenica, I will use the terms information design and Bayesian persuasion interchangeably, even though the former usually refers to models with multiple receivers and the latter to models with a single receiver.

${ }^{4}$ Tsakas et al. (2021) provide a model where the receiver has some commitment power in the sense of committing to not choosing certain actions.

${ }^{5}$ We adapt a well known example in game theory for coordination games, Battle of the Sexes (aka Bach or Stravinsky), to a Bayesian persuasion context.
} 
(that is contingent on the true state of the world) a communication strategy. ${ }^{6}$ Alex chooses $\pi$ and truthfully reports the realization from $\pi$ to Bob.

If the research is totally uninformative, then Bob's belief does not change and he will want to go to a Stravinsky concert (since he believes that orchestra is the high quality one with a higher probability). This totally uninformative research could be represented by $\pi^{0}$ as follows.

\begin{tabular}{c|cc}
$\pi^{0}$ & high & low \\
\hline$h$ & $\frac{1}{2}$ & $\frac{1}{2}$ \\
$\ell$ & $\frac{1}{2}$ & $\frac{1}{2}$
\end{tabular}

Here, $h$ and $\ell$ are realizations of $\pi^{0}$, which can be thought of as getting a signal that the orchestra is high and low quality, respectively. Here, no matter what the true state of the world is, Bob observes a signal that the orchestra is high quality with probability $1 / 2$, which means Bob learns nothing from $\pi^{0}$.

In contrast, if Alex does a fully informative research, then Bob chooses to go to a Bach concert a third of the time. This communication strategy $\pi^{1}$ can be represented as follows.

\begin{tabular}{c|cc}
$\pi^{1}$ & high & low \\
\hline$h$ & 1 & 0 \\
$\ell$ & 0 & 1
\end{tabular}

In other words, Bob observes $h$ with probability 1 if the orchestra is high quality and $\ell$ with probability 1 if the orchestra is low quality; Bob knows the quality of the orchestra after observing a message. Alex, however, can improve his chances of going to a Bach concert by garbling information when the true state is low and using the following communication strategy.

\begin{tabular}{c|cc}
$\pi$ & high & low \\
\hline$h$ & 1 & $\frac{1}{2}$ \\
$\ell$ & 0 & $\frac{1}{2}$
\end{tabular}

By communicating via $\pi$, Alex is restricting some information to Bob. While if the orchestra is high quality Bob observes $h$ with probability 1 , he observes $\ell$ with only probability $1 / 2$ if the orchestra is low quality. Bob, knowing that some information is not being provided to him, is still persuaded to go to a Bach concert upon observing realization $h$ (since he has a posterior belief of $1 / 2$ ). The probability of Bob observing $h$ under $\pi$ is $1 / 3 \cdot 1+2 / 3 \cdot 1 / 2=2 / 3$.

\footnotetext{
${ }^{6}$ The distribution $\pi$ has various names in the literature such as signal, experiment, and communication protocol. We chose to call $\pi$ a communication strategy and an element of the set of realizations of $\pi$ a signal.
} 
Thus, even though Bob initially believes that the orchestra is high quality with probability $1 / 3$, he agrees to go to a Bach concert two thirds of the time.

When there is a single receiver as in the example above, the unique optimal communication strategy is given by $\pi$. The ease of extending these results to multiple receivers depends on several factors. The following passage from Kamenica and Gentzkow (2011) highlights these factors:

"There are two classes of multiple-receiver models where our results can be extended quite easily. The first is one where the sender sends separate (possible correlated) messages to each receiver, the sender's utility is separable across receivers' actions, and each receiver cares only about her own action. In this case, we can simply apply our approach separately to the sender's problem vis-à-vis each receiver. The second class is where the sender can persuade only by revealing public information."

The focus of this dissertation is mainly on voting applications of Bayesian persuasion (along with an analysis on feasible distributions and informativeness of communication strategies). This means that the models involve collective decision making, which implies that each receiver does not only care about his own action, but about the outcome of the vote. Furthermore, the sender's utility is not separable across receivers' actions as the sender only wants the proposal to pass. Thus, our extension does not fall into the first class of multiple receiver models mentioned in Kamenica and Gentzkow (2011). Neither does it fall under the second class that is mentioned, since we are interested in private communication. Consequently, it is not a trivial problem to consider collective decision making under private communication.

CHAPter 2 focuses on targeted private communication in elections, where a sender (e.g. an incumbent party) is trying to persuade an electorate (identical receivers) to pass a proposal (i.e. there are two states of the world, the proposal being "good" or "bad" for society). There are two main approaches in modelling voter behavior, sincere and strategic voting; we assume that voters vote sincerely, that is, taking into account only their own beliefs. This chapter is primarily motivated by the fact that under an optimal communication strategy, sincere voting does not constitute a Bayesian Nash equilibrium (BNE). More precisely, we observe the swing voter's curse, that is, receivers are better off voting against their beliefs, or in other words, they are better off not following the sender's recommendation. Hence, as opposed to the standard BNE approach, we employ sincere voting as the equilibrium refinement and characterize an optimal communication strategy that leads to an equilibrium under sincere voting. We show that the sender can still do better than public communication when she implements sincere voting as a BNE, but the probability of success is lower than the case where we take sincere voting as given. 
Chapter 3 considers a natural extension to the set-up of CHAPTER 2. It is often assumed in Bayesian persuasion models that receivers do not communicate with each other prior to taking their action. In reality, however, we know that this is often not the case; voters deliberate with each other before voting, customers ask opinions of each other about a product before purchasing, and so on. Taking communicating voters into account, what would the sender's optimal communication strategy look like? Would the sender always prefer to have voters who do not communicate? Or are there cases where the sender's ability to manipulate the voters increases with more communication? These are some of the questions we tackle in Chapter 3. We incorporate communicating receivers by assuming that they are in a commonly known communication network, in which they can observe their neighbors' private messages (i.e. we have perfect exchange of information among neighbors). While the network structure complicates the problem (in the sense that our ability to simplify the problem is hindered), we can identify optimal communication for different types of networks (e.g. line, star, circle). The sender does, in fact, prefer voters who do not communicate (i.e. the empty network), however, there exist network types under which the sender can do as well as the case of non-communicating voters. Furthermore, perhaps surprisingly, in some non-empty networks, it is possible for the sender to fare better if links are added. That is, more communication in the network is not always necessarily bad for the sender.

ChApter 4 refrains from a voting application and focuses on feasibility and informativeness of beliefs. In Bayesian persuasion problems, the sender's payoff is often determined only by the beliefs of the receivers. Hence, this makes determining the feasibility (or inducibility) of distributions of beliefs an important problem in information design. While inducible beliefs are characterized by only one condition (Bayes plausibility) when there is a single receiver, this condition is insufficient when there are multiple receivers. We consider a multiple-receiver model and determine the additional conditions required for inducibility, i.e., we provide a characterization of inducible beliefs (for a large and finite state space). Given an inducible distribution of beliefs, we explore different classes of communication strategies that induce this distribution, and introduce a new order on the set of communication strategies to compare their informativeness. In other words, we are interested in the informativeness of communication strategies that induce the same first-order beliefs, but differ in the higher order information they provide. This notion of informativeness becomes particularly useful if a sender wishes to restrict the information the receivers have about each others' beliefs about the true state (e.g. a spy trying to create disagreement between two political parties). We then provide a characterization for the least informative communication strategy that induces a given distribution of beliefs. 



\section{Chapter 2}

\section{Bayesian Persuasion in Elections with Sincere Voting}

"I'd rather vote for something I want and not get it than vote for something I don't want, and get it." 
In this chapter, we consider a sender who wants to persuade multiple receivers to vote in favor of a proposal. Prior to the vote the sender commits to a communication strategy which sends private, potentially correlated, signals to the receivers that are contingent on the true state of the world. While the sender benefits from using private messages rather than public communication if the receivers vote sincerely, under the optimal private communication strategy, sincere voting is not in any receiver's best interest. We characterize the optimal private communication strategy conditional on sincere voting being a Bayes Nash equilibrium and show that the sender still benefits from private communication.

\subsection{Introduction}

Suppose a country is holding a referendum in order to decide whether or not to adopt a certain policy. Then, typically, government has a strict preference for either the proposal or the status quo. For instance, in the run up to the Brexit referendum, a majority of the cabinet campaigned against leaving the European Union (that is, in favor of the status quo), ${ }^{1}$ whereas in the 2017 Turkish constitutional referendum government campaigned for replacing the existing parliamentary system with a presidential system (that is, in favor of the proposal). Either way, the aim of a political campaign run by government is to increase the probability that their preferred outcome obtains a majority of the votes, that is, to persuade the electorate.

Both the Brexit referendum and the Turkish constitutional referendum are examples of a voting game. In voting games, a myriad of trivial (and unappealing) equilibria emerge due to coordination failures. For instance, in a simple majority game all voters voting for their least preferred option is an equilibrium as no voter can affect the outcome by changing their vote. In the literature (Osborne and Slivinski, 1996; Banks and Duggan, 2000; Levy, 2004) typical solutions to such coordination failures assume sincere voting, that is, each Receiver votes for the alternative which would give the highest utility if implemented, or use an equilibrium refinement such as trembling hand perfection. In models where receivers are symmetrically informed, both approaches result in the same equilibrium selection, but in general they may not. In this paper, we focus on sincere voting because of its tractability and empirical relevance. Poole and Rosenthal (2000) and Groseclose and Milyo (2010) show that strategic voting is rare in Congress, Felsenthal and Brichta (1985) and Degan and Merlo (2007) argue that sincere voting is consistent with voter behavior in larger elections, where numerous papers provide explanations for such behavior (Denzau et al., 1985; Austen-Smith, 1992; Kleiner and Moldovanu, 2017).

\footnotetext{
${ }^{1}$ https://www.bbc.com/news/uk-politics-eu-referendum-35616946
} 


\subsubsection{Overview of Results}

We consider a sender who wishes to persuade multiple Receivers to vote in favor of a proposal. Receivers' utility is determined by the unknown state of the world and the outcome that is being implemented. The sender commits to a communication strategy and reveals information about the true state, after which each Receiver sincerely votes either in favor or against the proposal. The proposal is implemented if it is approved by at least $k$ Receivers.

We first consider public communication, that is, all Receivers observe the same message under the sender's communication strategy. We show that in this case the sender's optimal communication strategy follows from Kamenica and Gentzkow (2011). The sender, however, can implement the proposal with a higher probability under private communication, where Receivers know the joint signal distribution, but only observe their own private message. ${ }^{2}$ The sender's optimal private communication strategy prescribes sending a recommendation to vote in favor of the proposal to all Receivers if the proposal is indeed beneficial (henceforth state $X$ ). If the proposal is harmful (henceforth state $Y$ ), then the recommendation to vote in favor of the proposal is sent either only to $k$ agents (in other words to a minimal winning coalition) or to none.

The structure of the optimal communication strategy implies that a Receiver is pivotal only when he has observed the recommendation to vote in favor of the proposal and when the state is $Y$. It follows that a Receiver has an incentive to vote against his recommendation; sincere voting is not a Bayes Nash equilibrium (BNE). In particular, we observe the swing voter's curse (Feddersen and Pesendorfer, 1996), which refers to situations in which a partially informed voter is pivotal only when he should vote against his favorite outcome. ${ }^{3}$

The swing voter's curse implies that a Receiver is pivotal only if the state is $Y$. Thus, starting from the optimal communication strategy, there are two ways to make sincere voting a BNE and avoid the swing voter's curse: $(i)$ by increasing the probability of being pivotal in $X$ or $(i i)$ by decreasing the probability of being pivotal in $Y$. It turns out that the optimal communication strategy which makes sincere voting a BNE follows the second route. In particular, by sending a recommendation to vote in favor of the proposal to $k+1$ Receivers or none in state $Y$, for each Receiver, the probability of being pivotal is reduced to zero. As a single Receiver cannot change the outcome of the vote, he has no incentive to deviate from his recommendation and thus, sincere voting is a BNE.

The rest of the paper is organized as follows. In Section 2.1.2 we provide an illustrative example and in Section 2.1.3 we give a review of related literature. In Section 2.2 we introduce

\footnotetext{
${ }^{2}$ Note that public communication is a special case of private communication where all private messages are the same.

${ }^{3}$ There are experimental studies on the swing voter's curse such as Battaglini et al. (2010) and Grosser and Seebauer (2016). For a both theoretical and experimental approach on the swing voter's curse in the context of communication with experts, see Buechel and Mechtenberg (2019).
} 
the necessary notation, define the voting game, and formulate the sender's problem. In Section 2.3 we first show that the sender can restrict attention to straightforward communication strategies and then find the optimal communication strategy. In Section 2.4 we define BayesNash equilibrium and in Section 2.5, we find the optimal communication strategy which makes sincere voting a BNE. Finally, we conclude in Section 2.6. Appendix 2.7.1 presents two useful lemmas related to straightforwardness. All proofs can be found in Appendix 2.7.2.

\subsubsection{An Illustrative Example}

Consider a government (the sender) who proposes to ban cars on certain days of the year. For simplicity, assume that changing the status quo requires the approval of three out of five committee members (the receivers). Committee members are not certain whether the proposal will benefit $(X)$ or harm $(Y)$ society and vote in favor of the proposal if they think that the chance of $X$ is at least $50 \%$. Government wants the proposal to pass independently of its benefit or harm and, hence, wants to persuade voters to approve the proposal. Government and committee members have a common prior belief that the ban will be beneficial with $30 \%$ probability. If committee members vote sincerely, then, without intervention, no committee member will vote in favor of the ban and the ban will not be implemented.

Fully informative research Suppose that prior to the vote, government attempts to influence the committee by conducting research and truthfully sharing the results with the committee members. Government can, for example, ask political scientists and economists to report their findings on the possible implications of implementing the ban. Given the common prior, there is a 30\% chance that this research will prove the ban on cars to be beneficial. Hence, by conducting this research, government can increase the ex ante probability of implementing the ban to $30 \%$.

Persuasion with public signals Government can, however, improve upon this by conducting a partially informative research whose results will be shared publicly. This could be done, for example, by hiring a partisan economist whose research only points out the benefits of implementing the ban. Note that since all members' observations are the same, either all members are persuaded or none of them is; the optimal public communication strategy follows from the single receiver case in Kamenica and Gentzkow (2011): all committee members receive message $x$ with probability 1 in state $X$ and with probability $3 / 7$ in $Y$. The optimal communication strategy in this case increases the acceptance probability of the proposal to $60 \%$. 
Persuasion with private correlated signals Capabilities of government do not end here; it can do better by using correlated private messages. Let government's research be formalized by distributions $\pi^{*}(\cdot \mid X)$ and $\pi^{*}(\cdot \mid Y)$ on some set of signals. Suppose that government hires two economists, one being honest and one partisan. The honest economist conducts a fully informative study and reports to two randomly selected committee members. The partisan conducts a biased study that will send $x$ if $X$ is the true state and send $x$ with probability $5 / 7$ if $Y$ is the true state. He reports his results to the remaining three committee members. The following table captures the conditional probabilities for each of the twelve possible signal vectors being sent.

\begin{tabular}{c|cc}
$\pi^{*}$ & $\omega=X$ & $\omega=Y$ \\
\hline$(x, x, x, x, x)$ & 1 & 0 \\
$(x, x, x, y, y)$ & 0 & $\frac{1}{14}$ \\
$(x, x, y, y, x)$ & 0 & $\frac{1}{14}$ \\
$(x, y, y, x, x)$ & 0 & $\frac{1}{14}$ \\
$(y, y, x, x, x)$ & 0 & $\frac{1}{14}$ \\
$(y, x, y, x, x)$ & 0 & $\frac{1}{14}$ \\
$(y, x, x, y, x)$ & 0 & $\frac{1}{14}$ \\
$(y, x, x, x, y)$ & 0 & $\frac{1}{14}$ \\
$(x, y, x, y, x)$ & 0 & $\frac{1}{14}$ \\
$(x, x, y, x, y)$ & 0 & $\frac{1}{14}$ \\
$(x, y, x, x, y)$ & 0 & $\frac{1}{14}$ \\
$(y, y, y, y, y)$ & 0 & $\frac{2}{7}$
\end{tabular}

With $\pi^{*}$, government can increase the probability of implementing its preferred outcome to $80 \%$. We will show later on that $\pi^{*}$ is indeed optimal.

The swing voter's curse and the BNE constraint In the previous scenario committee members know that the ban will be harmful to society upon receiving $y$, but regard the states equally likely upon receiving $x$. Given communication strategy $\pi^{*}$, if all voters are sincere, each one becomes pivotal if and only if two conditions are satisfied: ( $i$ ) they have received $x$ and (ii) the ban is harmful. Thus, upon observing $x$ choosing $y$ is optimal if all other voters vote sincerely. This means that sincere voting is not a BNE. It is, then, natural to ask: what can the sender achieve under the additional constraint that sincere voting be a BNE?

The optimal private communication strategy under which sincere voting is a BNE (denoted $\pi^{* \mathrm{e}}$ ) reduces the probability of being pivotal to zero by sending $x$ to four out of five committee members, as opposed to sending it to a minimal winning coalition. It follows that a single committee member cannot change the outcome of the vote, in other words, sincere voting 
is optimal. The following table captures the conditional probabilities for each of the seven possible signal vectors being sent.

\begin{tabular}{c|cc}
$\pi^{* \mathrm{e}}$ & $\omega=X$ & $\omega=Y$ \\
\hline$(x, x, x, x, x)$ & 1 & 0 \\
$(x, x, x, x, y)$ & 0 & $\frac{3}{28}$ \\
$(x, x, x, y, x)$ & 0 & $\frac{3}{28}$ \\
$(x, x, y, x, x)$ & 0 & $\frac{3}{28}$ \\
$(x, y, x, x, x)$ & 0 & $\frac{3}{28}$ \\
$(y, x, x, x, x)$ & 0 & $\frac{3}{28}$ \\
$(y, y, y, y, y)$ & 0 & $\frac{13}{28}$
\end{tabular}

With $\pi^{* e}$, government can implement its preferred outcome with $67.5 \%$ probability. It follows that having committee members vote sincerely in equilibrium is not without a cost: with the additional constraint that sincere voting be a BNE, the probability of implementing $x$ in state $Y$ under the optimal communication strategy is $15 / 28$, while this probability is $5 / 7$ under $\pi^{*}$. However, as this is still higher than the probability under the optimal public communication strategy, government benefits from private communication.

\subsubsection{Related Literature}

There are several papers in the Bayesian persuasion literature which extend Kamenica and Gentzkow (2011) by considering private communication between a sender and multiple receivers. In a closely related paper, Chan, Gupta, Li, and Wang (2019) consider a setting with costly voting and show that the optimal communication strategy targets minimal winning coalitions; in particular, the receivers who are easiest to persuade. It follows that in equilibrium all receivers are pivotal with probability 1 . This is in stark contrast with our communication strategy under which sincere voting is a BNE: while receivers are pivotal in equilibrium when voting is costly, no receiver is ever pivotal in equilibrium when there is no cost of voting. The optimal communication strategy without the BNE constraint, on the other hand, resembles the optimal communication strategy of Chan et al. (2019) in the sense that receivers are pivotal with positive probability in the "bad" state. However, under our communication strategy receivers can be pivotal only in the bad state and they are pivotal with a probability less than 1 .

Our paper also relates to the literature on voting games and public communication. Schnakenberg (2015) considers an "expert" who can conceal information after she is privately informed about the state. He shows that the removal of the commitment assumption leads to stricter conditions on persuasion and that experts can manipulate information such 
that ex ante expected utilities of the voters are reduced. Alonso and Câmara (2016) consider a symmetric information voting model. They show that the optimal communication strategy exploits the heterogeneity of preferences of voters by targeting minimal winning coalitions.

As we extend public communication by allowing for private messages, our paper relates to papers which consider private communication under collective decision making. Wang (2013) studies both public and private communication with general voting rules. Under private communication, she focuses on perfectly uncorrelated signals and shows that the sender is weakly worse off under private persuasion. In contrast, we allow for correlated signals and show that the sender can improve upon public communication. Heese and Lauermann (2017) consider private communication in large elections and allow for exogenous private signals for receivers. They show that any state-contingent outcome can be implemented in some BNE. Bardhi and Guo (2018) consider voters with correlated payoff states and focus on unanimity voting, while we allow for more general voting rules.

A paper which does not consider a voting game but is still related to ours is Arieli and Babichenko (2019). While they also consider private communication with multiple receivers, the sender in their model wishes to maximize the number of adoptions of a product, whereas the sender in our model tries to influence a collective decision. In particular, they characterize the optimal communication strategy for different utility functions of the sender. For the case where we do not impose the BNE constraint on the communication strategy, we can relate our optimal communication strategy to theirs.

Our paper is also linked to a literature on information design in more general games. Bergemann and Morris (2016a) study a game of incomplete information with multiple receivers and show that the set of outcomes that can arise in BNE corresponds to Bayes correlated equilibrium outcomes. Bergemann and Morris (2016a) allow for receivers to observe additional private signals, whereas in a related paper Taneva (2019) focuses on a sender who has full control over the information gathered by the receivers. While we consider a more specific setup, namely voting games, we also characterize the optimal communication strategy under BNE. Mathevet, Perego, and Taneva (2020) consider an epistemic approach, incorporating higher order beliefs into persuasion, whereas we consider receivers who behave according to their first order beliefs and are interested in situations where it is optimal for them to do so.

We assume neither the sender nor the receivers to have any exogenous private information. In single receiver information design problems, this extension has been explored by several authors. Hedlund (2017) considers a privately informed sender and characterizes equilibria which are either separating or fully disclosing. In a closely related paper Kosenko (2018) considers a simpler model which explores how particular assumptions affect the sender's expected utility. In contrast, Tsakas and Tsakas (2017) consider a model with a privately informed receiver, where there is noise due to exogenous information distortions and show that more noise is often beneficial for the sender. Kolotilin, Mylovanov, Zapechelnyuk, and Li (2017) 
consider a sender who communicates with a privately informed receiver and who discloses information either by choosing a persuasion mechanism (which depends on Receiver type) or an experiment (which is independent of Receiver type) and establish the equivalence of the two communication types. While we believe that similar extensions would be a fruitful direction for future research in our set-up, such considerations are outside the scope of this paper.

\subsection{Preliminaries and Notation}

\subsubsection{Communication Strategies and Beliefs}

Let $N=\{1, \ldots, n\}$ be the set of Receivers and let $\Omega$ be a finite set of states of the world. Let $S_{i}$ be a finite set of messages the sender can send to receiver $i \in N$ and let $S=\prod_{i \in N} S_{i}$ be the set of signals. A communication strategy is a function $\pi: \Omega \rightarrow \Delta(S)$ which maps each state of the world to a joint probability distribution over signal realizations. Denote the set of all communication strategies by $\Pi$. For each signal $s \in S$, let $s_{i} \in S_{i}$ denote the message for Receiver $i$. For each $s_{i}^{\prime} \in S_{i}$ and $\omega \in \Omega$, let

$$
\pi_{i}\left(s_{i}^{\prime} \mid \omega\right)=\sum_{s \in S: s_{i}=s_{i}^{\prime}} \pi(s \mid \omega)
$$

which is the probability that Receiver $i$ observes $s_{i}$ given that the true state is $\omega$. Note that $\pi_{i}\left(s_{i}^{\prime} \mid \omega\right)$ is a marginal probability and is obtained by summing the probabilities of signals in which $i$ observes $s_{i}$. For any $\pi \in \Pi$ define $S^{\pi}=\{s \in S \mid \exists \omega \in \Omega: \pi(s \mid \omega)>0\}$. That is, $S^{\pi}$ consists of signals in $S$ which are sent with positive probability by $\pi$. Similarly, for each $\pi \in \Pi$ and $i \in N$, define $S_{i}^{\pi}=\left\{s_{i} \in S_{i} \mid \exists \omega \in \Omega: \pi_{i}\left(s_{i} \mid \omega\right)>0\right\}$ to be the set of messages Receiver $i$ observes with positive probability under $\pi$.

For any set $X$ denote by $\Delta(X)$ the set of probability distributions over $X$ with finite support. Throughout the paper we assume that the sender and the receivers share a common prior belief $\lambda^{0} \in \Delta^{\circ}(\Omega)$ about the true state of the world, where $\Delta^{\circ}(\Omega)$ denotes the set of strictly positive probability distributions on $\Omega$. Given $\lambda^{0} \in \Delta^{\circ}(\Omega)$ and $\pi \in \Pi$, a signal $s \in S^{\pi}$ generates the posterior belief vector $\lambda^{s} \in \Delta(\Omega)^{n}$ defined by

$$
\lambda_{i}^{s}(\omega)=\frac{\pi_{i}\left(s_{i} \mid \omega\right) \lambda^{0}(\omega)}{\sum_{\omega^{\prime} \in \Omega} \pi_{i}\left(s_{i} \mid \omega^{\prime}\right) \lambda^{0}\left(\omega^{\prime}\right)}, \quad i \in N, \omega \in \Omega .
$$

That is, $\lambda_{i}^{s}(\omega)$ denotes $i$ 's updated belief upon receiving $s_{i}$ that the true state is $\omega$. Let $\sigma \in \Delta\left(\Delta(\Omega)^{n}\right)$ be a distribution over belief vectors. A communication strategy $\pi \in \Pi$ induces $\sigma$ if for all $\lambda \in \operatorname{supp}(\sigma)$ it holds that

$$
\sigma(\lambda)=\sum_{s \in S^{\pi}: \lambda^{s}=\lambda} \sum_{\omega \in \Omega} \pi(s \mid \omega) \lambda^{0}(\omega)
$$


In words, $\sigma(\lambda)$ is the probability of posterior vector $\lambda .{ }^{4}$ We say that $\sigma$ is inducible if there exists a communication strategy which induces it. As $S$ is finite, each inducible $\sigma$ is a finite distribution.

\subsubsection{Voting Problems}

A voting problem is a tuple $\left(N, \Omega, Z,\left(A_{i}\right)_{i \in N}, k, v,\left(u_{i}\right)_{i \in N}, \lambda^{0}\right)$ of the set of Receivers $N$, the set of states $\Omega$, the set of outcomes $Z$, Receivers' action sets $A_{i}$ that determine the outcome together with integer quota $k \geq 1$, the sender's utility function $v: Z \times \Omega \rightarrow \mathbb{R}$, Receivers' utility functions $u_{i}: Z \times \Omega \rightarrow \mathbb{R}$, and a common prior $\lambda^{0}$.

From here we shall focus on a binary set of states, that is, $\Omega=\{X, Y\}$. We assume that there are two voting outcomes which we represent by $Z=\{x, y\}$ and that there are two possible actions for each Receiver. That is, for each $i \in N$ we assume $A_{i}=\{x, y\}$. Action $x$ means casting a vote in favor of $x$ and action $y$ a vote in favor of $y$.

Let $A=\prod_{i \in N} A_{i}$ be the space of action profiles. Let $z^{k}: A \rightarrow Z$ be the voting rule such that for each $a \in A$, we have

$$
z^{k}(a)= \begin{cases}x & \text { if }\left|i \in N: a_{i}=x\right| \geq k \\ y & \text { otherwise }\end{cases}
$$

So, $z^{k}(a)$ is the outcome of the vote when the action profile is $a$ and the quota is $k .{ }^{5}$ If $k=n$, then $z^{k}$ is the unanimity rule.

We assume that the sender's utility is independent of the state and only depends on the outcome. For any $z \in Z$, the sender's utility function is defined as

$$
v(z)= \begin{cases}1 & \text { if } z=x \\ 0 & \text { otherwise }\end{cases}
$$

That is, the sender wants to implement $x$ and does not care about the true state.

For each $i \in N$, let $u_{i}: Z \times \Omega \rightarrow\{0,1\}$ be the utility function of Receiver $i$, where $u_{i}(z, \omega)$ is the utility of $i$ when the implemented outcome is $z$ and the true state is $\omega$. We assume that

$$
u_{i}(z, \omega)= \begin{cases}1 & \text { if }[z=x \text { and } \omega=X] \text { or }[z=y \text { and } \omega=Y] \\ 0 & \text { otherwise }\end{cases}
$$

That is, Receivers want the implemented outcome to match the true state of the world.

\footnotetext{
${ }^{4}$ Note that if $\lambda$ does not belong to $\operatorname{supp}(\sigma)$, then the equality in (2.1) remains valid as then the right-hand side is equal to 0 .

${ }^{5}$ Note that any anonymous and monotonic voting rule is of this form.
} 


\subsubsection{Persuasion}

As pointed out in the literature review, assuming that voters vote sincerely is a common way to avoid an unappealing multiplicity of equilibria. We follow this approach in our paper as well. Let a communication strategy $\pi \in \Pi$ be given. For each $i \in N$, let $\alpha_{i}^{\pi}: S_{i}^{\pi} \rightarrow A_{i}$ be the sincere action function of Receiver $i$. That is, $\alpha_{i}^{\pi}$ assigns the action that a sincere voter $i$ would choose upon observing $s_{i}$. More precisely, let

$$
\alpha_{i}^{\pi}\left(s_{i}\right)= \begin{cases}x & \text { if } \lambda_{i}^{s}(X) \geq \frac{1}{2} \\ y & \text { otherwise }\end{cases}
$$

Note that $\alpha_{i}^{\pi}$ is the "Sender-preferred" sincere action function: receivers vote in favor of the sender's preferred outcome in case they are indifferent. In particular, if $\lambda^{0}(X) \geq 1 / 2$, then interests of the sender and the receivers are aligned and there is no need for communication.

Let $\pi \in \Pi$ be a communication strategy and for any $i \in N$ let

$$
\begin{aligned}
& M_{i}^{x}(\pi)=\left\{m \in S_{i}^{\pi} \mid \alpha_{i}^{\pi}(m)=x\right\}, \\
& M_{i}^{y}(\pi)=\left\{m \in S_{i}^{\pi} \mid \alpha_{i}^{\pi}(m)=y\right\},
\end{aligned}
$$

which are the sets of messages in $S_{i}^{\pi}$ which make $i$ choose $x$ and $y$, respectively.

We say that Receiver $i$ is pivotal in $s \in S^{\pi}$ if for any $a_{i} \in A_{i}, z^{k}\left(a_{i}, \alpha_{-i}^{\pi}\left(s_{-i}\right)\right)=a_{i}$. That is, $i$ is pivotal in the voting game following $s$ if $i$ 's vote determines the voting outcome given that all $j \neq i$ vote sincerely. Define

$$
Z^{x}(\pi)=\left\{s \in S^{\pi} \mid z^{k}\left(\alpha^{\pi}(s)\right)=x\right\}
$$

as the set of signals in $S^{\pi}$ that lead to $x$ being implemented under sincere voting. The sender's expected utility given $\lambda^{0} \in \Delta^{\circ}(\Omega)$ and $\pi \in \Pi$ is the value of $\pi$ at $\lambda^{0}$ and denoted by $V^{\pi}\left(\lambda^{0}\right)$. The value $V^{\pi}\left(\lambda^{0}\right)$ equals the probability that $x$ is being implemented given $\lambda^{0}$ and $\pi$. That is,

$$
V^{\pi}\left(\lambda^{0}\right)=\mathbb{E}_{\lambda^{0}}\left[\mathbb{E}_{\pi}\left[v\left(z^{k}\left(\alpha^{\pi}(s)\right)\right)\right]\right]=\lambda^{0}(X) \sum_{s \in Z^{x}(\pi)} \pi(s \mid X)+\lambda^{0}(Y) \sum_{s \in Z^{x}(\pi)} \pi(s \mid Y) .
$$

Let $\Pi^{\prime} \subseteq \Pi$. The optimal value at $\lambda^{0}$ in $\Pi^{\prime}$ is

$$
\sup _{\pi \in \Pi^{\prime}} V^{\pi}\left(\lambda^{0}\right)
$$

We say that $\pi^{*} \in \Pi^{\prime}$ is optimal in $\Pi^{\prime}$ at $\lambda^{0} \in \Delta^{\circ}(\Omega)$ if $V^{\pi^{*}}\left(\lambda^{0}\right)=\max _{\pi \in \Pi^{\prime}} V^{\pi}\left(\lambda^{0}\right)$. Note that for general $\Pi^{\prime}$ such $\pi^{*}$ might not exist. Nevertheless, the various classes of communication strategies that we shall consider later all have an optimal element. 


\subsection{Optimal Communication}

We start by giving a very simple example of how private communication improves upon public communication under the assumption that Receivers vote sincerely.

Example 2.1. Let $N=\{1,2\}, k=1$, and $\lambda^{0}(X)=1 / 3$. For each $i \in N$, let $S_{i}=\{x, y\}$ be the set of messages. It follows from the analysis in Kamenica and Gentzkow (2011) that the optimal public communication strategy $\pi^{* \mathrm{p}}$ is given by

\begin{tabular}{c|cc}
$\pi^{* \mathrm{p}}$ & $\omega=X$ & $\omega=Y$ \\
\hline$(x, x)$ & 1 & $\frac{1}{2}$ \\
$(y, y)$ & 0 & $\frac{1}{2}$
\end{tabular}.

Under $\pi^{* \mathrm{p}}$, persuading only one of the Receivers is not possible, in other words either both Receivers are persuaded or none is. The value in this case is $V^{\pi^{* \mathrm{p}}}\left(\lambda^{0}\right)=1 / 3 \cdot 1+2 / 3 \cdot 1 / 2=2 / 3$. The optimal private communication strategy $\pi^{*}$, however, allows for persuading a single agent which improves the probability of success.

\begin{tabular}{c|cc}
$\pi^{*}$ & $\omega=X$ & $\omega=Y$ \\
\hline$(x, x)$ & 1 & 0 \\
$(x, y)$ & 0 & $\frac{1}{2}$ \\
$(y, x)$ & 0 & $\frac{1}{2}$
\end{tabular}.

Note that $x$ is implemented after any signal realization. Thus, $V^{\pi^{*}}\left(\lambda^{0}\right)=1$.

Both in Example 2.1 and in our motivating example of a government wanting to ban cars on certain days of the year, the messages Receivers observe are recommendations to either vote in favor of or against the proposal, that is, for each $i \in N, S_{i}^{\pi} \subseteq A_{i}$. However, in the case of the motivating example, it could also be that messages are statistics about the pollution caused by excess car usage on which Receivers base their decision, rather than a recommended action, so that $S_{i}^{\pi}$ would not be a subset of $A_{i}$. In Section 3.1 we show that we can without loss of generality assume $S_{i}^{\pi} \subseteq A_{i}$ for all $i \in N$ when searching for the optimal communication strategy of the sender.

\subsubsection{Straightforward Communication Strategies}

Definition 2.2. A communication strategy $\pi \in \Pi$ is straightforward if for all $i \in N$

(i) $S_{i}^{\pi} \subseteq A_{i}$,

(ii) $\alpha_{i}^{\pi}\left(a_{i}\right)=a_{i}$ for all $a_{i} \in A_{i}$. 
Denote the set of all straightforward communication strategies by $\Pi^{\mathrm{s}}$. Under a straightforward communication strategy, for each $i \in N$, the set of messages $i$ can observe with positive probability is a subset of his action set; thus, the communication strategy sends a "recommended" action to each Receiver. Condition (ii) requires that these recommendations be convincing in the sense that sincere voters follow them.

By definition, a communication strategy $\pi \in \Pi$ with $S^{\pi} \subseteq\{x, y\}^{n}$ is straightforward if for any $i \in N$ and $s, t \in S^{\pi}$ with $s_{i}=x$ and $t_{i}=y$, we have $\lambda_{i}^{s}(X) \geq 1 / 2$ and $\lambda_{i}^{t}(Y)>1 / 2$. Hence, $\pi$ is straightforward if and only if for all $i \in N$

$$
\begin{aligned}
& \lambda^{0}(X) \pi_{i}(x \mid X) \geq \lambda^{0}(Y) \pi_{i}(x \mid Y), \\
& \lambda^{0}(X) \pi_{i}(y \mid X)<\lambda^{0}(Y) \pi_{i}(y \mid Y) .
\end{aligned}
$$

Note that if $\lambda^{0}(X)<1 / 2$, Equation (2.4) implies Equation (2.5), so that we don't need to pay special attention to the latter. We give a formal proof of this fact in Lemma 2.22 in Appendix A.

Although focusing on straightforward communication strategies simplifies the analysis, one important defect of doing so is the shrinkage in the set of inducible posteriors: it is not in general true that any inducible posterior can be induced by a straightforward communication strategy. In spite of this limitation of focusing on straightforward communication strategies, we will show that for the classes of communication strategies we are interested in, it is without loss of generality to do so when solving the optimization problem in (2.3). In Lemma 2.23 in Appendix 2.7.1 we show that although there might not always exist a straightforward communication strategy which induces the same distribution as any given $\hat{\pi} \in \Pi$, there always exists $\pi \in \Pi^{\mathrm{s}}$ which has at least the same value as $\hat{\pi}$.

\subsubsection{Optimal Public Communication}

We say that a communication strategy $\pi \in \Pi$ is public if for all $s \in S^{\pi}$ and all $i, j \in N, s_{i}=s_{j}$, that is, if all Receivers observe the same message under $\pi$. Since agents have homogeneous preferences, a public communication strategy either persuades all Receivers or none. Thus, the analysis of a multiple Receiver model with public communication strategies is very similar to the well-known analysis of the single Receiver model in Kamenica and Gentzkow (2011). Having that said, we proceed with a brief discussion of the former in order to allow for later references.

Let $\Pi^{\mathrm{p}}$ be the set of all public communication strategies. The most that the sender can achieve using public signals is

$$
V^{* \mathrm{p}}\left(\lambda^{0}\right)=\sup _{\pi \in \Pi^{\mathrm{p}}} V^{\pi}\left(\lambda^{0}\right)
$$


As before, it is of no of loss of generality to consider only public straightforward communication strategies, that is, those $\pi \in \Pi$ with $S^{\pi} \subseteq\{\mathbf{x}, \mathbf{y}\} .^{6}$

Proposition 2.3. Let $\lambda^{0} \in \Delta^{\circ}(\Omega)$. Let $\pi^{* \mathrm{p}}$ be given by

$$
\pi^{* \mathrm{p}}(s \mid \omega)= \begin{cases}1 & \text { if } s=\mathbf{x} \text { and } \omega=X, \\ \min \left\{\frac{\lambda^{0}(X)}{\lambda^{0}(Y)}, 1\right\} & \text { if } s=\mathbf{x} \text { and } \omega=Y, \\ \max \left\{1-\frac{\lambda^{0}(X)}{\lambda^{0}(Y)}, 0\right\} & \text { if } s=\mathbf{y} \text { and } \omega=Y .\end{cases}
$$

Then $\pi^{* \mathrm{p}}$ is optimal in $\Pi^{\mathrm{p}}$. In particular, $V^{* \mathrm{p}}\left(\lambda^{0}\right)=\min \left\{2 \lambda^{0}(X), 1\right\}$.

The proof of Proposition 2.3 follows easily from Kamenica and Gentzkow (2011). Via public communication the sender can achieve the value $\min \left\{2 \lambda^{0}(X), 1\right\}$. In the following sections we show how the sender can improve upon this by communicating privately with Receivers.

\subsubsection{Optimal Private Communication}

Our aim is to find a communication strategy which maximizes the sender's expected utility if the sender can use arbitrary private signals. In this case, the highest value she can achieve at any $\lambda^{0} \in \Delta^{\circ}(\Omega)$ is

$$
V^{*}\left(\lambda^{0}\right)=\sup _{\pi \in \Pi} V^{\pi}\left(\lambda^{0}\right)
$$

For the single Receiver case, Kamenica and Gentzkow (2011) show that it is optimal for the sender to send $x$ to the Receiver with probability 1 if the state is $X$. Lemma 2.4 shows that this result extends to the multiple Receiver case.

Lemma 2.4. Let $\lambda^{0} \in \Delta^{\circ}(\Omega)$ and $\hat{\pi} \in \Pi$. Then there is $\pi \in \Pi^{\mathrm{s}}$ such that for all $i \in N$ it holds that $\pi_{i}(x \mid X)=1$ and $V^{\pi}\left(\lambda^{0}\right) \geq V^{\hat{\pi}}\left(\lambda^{0}\right)$.

Note that Lemma 2.4 can also be derived from Lemma 1 of Arieli and Babichenko (2019): the proofs follow different approaches, nevertheless, the optimal communication strategy in both prescribes sending the "good" recommendation with probability 1 when the state is good.

Up until now, we showed that focusing on straightforward communication strategies and in particular, ones that send $x$ in state $X$ with probability 1 is without loss of generality. Furthermore, note that the voting rule $z^{k}$ is anonymous: only the total number of votes in favor of each outcome matters, not the identities of the voters who voted for each alternative. Hence, we might expect that communication strategies need not discriminate between Receivers either. In order to make this claim formal, let $B$ denote the set of all permutations on

\footnotetext{
${ }^{6}$ We denote by $\mathbf{x}, \mathbf{y} \in A$ the vectors $\left(x_{i}\right)_{i \in N}$ and $\left(y_{i}\right)_{i \in N}$, respectively.
} 
$N .^{7}$ For each $s \in S^{\pi}$ and each $b \in B$, denote by $s^{b}$ the unique signal vector with $s_{i}^{b}=s_{b(i)}$ for all $i \in N$. A communication strategy $\pi \in \Pi$ is called anonymous if $\pi(s \mid \omega)=\pi\left(s^{b} \mid \omega\right)$ for all bijections $b \in B$ and all $\omega \in \Omega$. The set of all straightforward and anonymous communication strategies is denoted by $\Pi^{\mathrm{sa}}$. In the next lemma we show that the sender can indeed restrict her search for the optimal communication strategy to $\Pi^{\text {sa }}$.

Lemma 2.5. Let $\lambda^{0} \in \Delta^{\circ}(\Omega)$ and $\hat{\pi} \in \Pi$. Then there is $\pi \in \Pi^{\mathrm{sa}}$ with $V^{\pi}\left(\lambda^{0}\right) \geq V^{\hat{\pi}}\left(\lambda^{0}\right)$.

Under an anonymous communication strategy, it does not matter who observes a message in a signal, only how many Receivers observe that message.

In the following, define for $\ell=0, \ldots, n$,

$$
S_{\ell}^{x}=\left\{s \in\{x, y\}^{n}:\left|\left\{j \in N: s_{j}=x\right\}\right|=\ell\right\} .
$$

That is, $S_{\ell}^{x}$ is the set of signals in which exactly $\ell$ Receivers observe $x$. An anonymous communication strategy $\pi$ can be represented by weights $q=\left(q_{0}, \ldots, q_{n}\right) \geq 0$ and $r=$ $\left(r_{0}, \ldots, r_{n}\right) \geq 0$ with $\sum_{\ell=0}^{n} q_{\ell}=\sum_{\ell=0}^{n} r_{\ell}=1$ such that for all $l=0, \ldots, n$ and all $s \in S_{l}^{x}$

$$
\pi(s \mid X)=\left(\begin{array}{l}
n \\
\ell
\end{array}\right)^{-1} q_{\ell} \quad \pi(s \mid Y)=\left(\begin{array}{c}
n \\
\ell
\end{array}\right)^{-1} r_{\ell} .
$$

That is, $q_{\ell}$ is the probability that $x$ is sent to exactly $\ell$ Receivers if the state is $X$ and $r_{\ell}$ is the probability that $x$ is sent to exactly $\ell$ Receivers if the state is $Y$. Thus, the probabilities that an agent observes $x$ given that the state is $X$ and $Y$, respectively, are

$$
\begin{aligned}
& \pi_{i}(x \mid X)=\sum_{\ell=0}^{n} \sum_{s \in S_{\ell}^{x}: s_{i}=x}\left(\begin{array}{l}
n \\
\ell
\end{array}\right)^{-1} q_{\ell}=\sum_{\ell=0}^{n}\left(\begin{array}{c}
n-1 \\
\ell-1
\end{array}\right)\left(\begin{array}{l}
n \\
\ell
\end{array}\right)^{-1} q_{\ell}=\sum_{\ell=0}^{n} \frac{\ell}{n} q_{\ell} \\
& \pi_{i}(x \mid Y)=\sum_{\ell=0}^{n} \sum_{s \in S_{\ell}^{x}: s_{i}=x}\left(\begin{array}{l}
n \\
\ell
\end{array}\right)^{-1} r_{\ell}=\sum_{\ell=0}^{n}\left(\begin{array}{c}
n-1 \\
\ell-1
\end{array}\right)\left(\begin{array}{l}
n \\
\ell
\end{array}\right)^{-1} r_{\ell}=\sum_{\ell=0}^{n} \frac{\ell}{n} r_{\ell} .
\end{aligned}
$$

We demonstrate the weights $q$ and $r$ in the next example.

Example 2.6. Let $n=3, k=2$, and $\lambda^{0}(X)=1 / 3$. Let $\pi^{*} \in \Pi$ be defined by weights $q_{3}^{*}=1$, $r_{0}^{*}=1 / 4$, and $r_{2}^{*}=3 / 4$. Alternatively, $\pi^{*}$ is given by

\begin{tabular}{c|cc}
$\pi^{*}$ & $\omega=X$ & $\omega=Y$ \\
\hline$(x, x, x)$ & 1 & 0 \\
$(x, x, y)$ & 0 & $\frac{1}{4}$ \\
$(x, y, x)$ & 0 & $\frac{1}{4}$ \\
$(y, x, x)$ & 0 & $\frac{1}{4}$ \\
$(y, y, y)$ & 0 & $\frac{1}{4}$
\end{tabular}

\footnotetext{
${ }^{7}$ A permutation on $N$ is a bijection $b: N \rightarrow N$.
} 
Recall that by Lemma 2.4, it is optimal to send $x$ to everyone with probability 1 if the state is $X$, that is, there is an optimal anonymous communication strategy with representation $(q, r)$ satisfying $q_{n}=1$. So, what remains is finding the optimal values for $r$. In the next proposition, we express $V^{*}\left(\lambda^{0}\right)$ as the solution to a simple linear optimization problem.

Proposition 2.7. Let $\lambda^{0} \in \Delta^{\circ}(\Omega)$. Then $V^{*}\left(\lambda^{0}\right)$ is the solution to

$$
\begin{aligned}
\max _{r_{0}, r_{k}} \lambda^{0}(X)+\lambda^{0}(Y) r_{k} & \\
\text { s.t. } r_{0} & \geq 0, \\
r_{k} & \geq 0, \\
r_{0}+r_{k} & =1, \\
\lambda^{0}(X)-\lambda^{0}(Y) \frac{k}{n} r_{k} & \geq 0 .
\end{aligned}
$$

The intuition behind Proposition 2.7 is as follows. Suppose the true state is $Y$ and message $x$ is sent to exactly $\ell$ Receivers with positive probability, where $\ell<k$. Under a straightforward communication strategy such a signal will not implement $x$ and can be replaced by $\mathbf{y}$. Suppose the true state is $Y$ and message $x$ is sent to exactly $\ell$ Receivers with positive probability, where $\ell>k$, so $x$ is implemented under a straightforward communication strategy. Sending $x$ to exactly $k$ Receivers would have the same effect without violating the straightforwardness constraint. Hence, choosing $r$ such that $r_{0}+r_{k}=1$ is without loss of generality.

By Lemma 2.5 and Proposition 2.7, for any $\lambda^{0} \in \Delta^{\circ}(\Omega)$, it holds that

$$
V^{*}\left(\lambda^{0}\right)=\sup _{\pi \in \Pi} V^{\pi}\left(\lambda^{0}\right)=\max _{\pi \in \Pi^{\mathrm{sa}}} V^{\pi}\left(\lambda^{0}\right)
$$

i.e., there is an anonymous and straightforward communication strategy that achieves $V^{*}\left(\lambda^{0}\right)$. Such an optimal communication strategy is given in Theorem 2.8.

Theorem 2.8. Let $\pi^{*} \in \Pi^{\mathrm{sa}}$ with representation $\left(q^{*}, r^{*}\right)$ be given by

$$
\left(q_{n}^{*} ; r_{0}^{*}, r_{k}^{*}\right)= \begin{cases}(1 ; 0,1) & \text { if } \lambda^{0}(X) \geq \frac{k}{n+k} \\ \left(1 ; 1-\frac{\lambda^{0}(X)}{\lambda^{0}(Y)} \frac{n}{k}, \frac{\lambda^{0}(X)}{\lambda^{0}(Y)} \frac{n}{k}\right) & \text { if } \lambda^{0}(X)<\frac{k}{n+k}\end{cases}
$$

Then $\pi^{*}$ is optimal at $\lambda^{0}$. In particular, $V^{*}$ is given by

$$
V^{*}\left(\lambda^{0}\right)=\min \left\{\frac{n+k}{k} \lambda^{0}(X), 1\right\} .
$$

The communication strategy $\pi^{*}$ differs from $\pi^{* \mathrm{p}}$ only if the state is $Y$. By sending $x$ to $k$ rather than $n$ receivers, the sender can increase the probability that at least $k$ voters observe $x$ without 
affecting each individual Receiver's probability of observing $x$, so the straightforwardness constraint remains satisfied. Since either $k$ voters observe $x$ or none does in state $Y$, Receivers are pivotal upon observing $x$.

If $|N|=3, k=2$, and $\lambda^{0}(X)=1 / 3$, then by Theorem 2.8 the optimal private communication strategy is given by $q_{3}^{*}=1, r_{0}^{*}=1 / 4$, and $r_{2}^{*}=3 / 4$, which is the communication strategy we considered in Example 2.6. Moreover, the sender is better off employing $\pi^{*}$ rather than $\pi^{* \mathrm{p}}$ as $V^{\pi^{*}}(1 / 3)=5 / 6>2 / 3=V^{\pi^{* \mathrm{p}}}(1 / 3)$.

We mentioned before that the optimal communication strategy resembles Chan et al. (2019) in the sense that it targets minimal winning coalitions. However, there is an important difference: while under their strategy Receivers are pivotal whenever they observe $x$, under ours Receivers are pivotal upon observing $x$ only when the state is $Y$.

\subsection{Swing Voter's Curse}

Recall the sincere voters from Example 2.6 and consider some fixed Receiver $i$. If the true state is $X$, all Receivers observe $x$ and, as they act sincerely, Receiver $i$ is not pivotal. If, on the other hand, the state is $Y$, he is pivotal upon observing $x$ as his vote will determine the outcome. Therefore, $i$ is better off choosing action $y$ upon observing $x$ : either the true state is indeed $X$ and his vote does not matter, or the state is $Y$ in which case voting for $y$ is strictly beneficial. This phenomenon has been coined the swing voter's curse: even though an agent believes that the true state is $X$, his rational action is to vote in favor of $y$. Note that under a public communication strategy there is no information asymmetry among voters and the swing voter's curse does not occur.

The following example illustrates the swing voter's curse by calculating Receiver's expected utility from voting $x$ after having observed $x$.

Example 2.9. Recall the communication strategy $\pi^{*}$ in Example 2.6. The expected utility of Receiver $i$ from voting for $x$ conditional on having observed message $x$ and provided that all other Receivers vote sincerely is given by

$$
\begin{aligned}
& \lambda_{i}^{\mathbf{x}}(X) \sum_{s \in S^{\pi}: s_{i}=x} \frac{\pi\left(\left(x, s_{-i}\right) \mid X\right)}{\pi_{i}(x \mid X)} u_{i}(x, X)+\lambda_{i}^{\mathbf{x}}(Y) \sum_{s \in S^{\pi}: s_{i}=x} \frac{\pi\left(\left(x, s_{-i}\right) \mid Y\right)}{\pi_{i}(x \mid Y)} u_{i}(x, Y) \\
& =\frac{1}{2} \cdot 1 \cdot 1+0=\frac{1}{2} .
\end{aligned}
$$

On the other hand, his expected utility from voting for $y$ conditional on having observing 
message $x$ and provided that all other Receivers vote sincerely is given by

$$
\begin{gathered}
\lambda_{i}^{\mathbf{x}}(X) \sum_{s \in S^{\pi}: s_{i}=x} \frac{\pi\left(\left(x, s_{-i}\right) \mid X\right)}{\pi_{i}(x \mid X)} u_{i}(x, X)+\lambda_{i}^{\mathbf{x}}(Y) \sum_{s \in S^{\pi}: s_{i}=x} \frac{\pi\left(\left(x, s_{-i}\right) \mid Y\right)}{\pi_{i}(x \mid Y)} u_{i}(y, Y) \\
=\frac{1}{2} \cdot 1 \cdot 1+\frac{1}{2} \cdot\left(\frac{1 / 4}{1 / 2} \cdot 1+\frac{1 / 4}{1 / 2} \cdot 1\right)=1 .
\end{gathered}
$$

Hence, choosing action y upon observing message $x$ is optimal for Receiver $i$.

Requiring that sincere voting indeed be in the voters' best interest poses a new constraint on the sender's optimization problem. This constraint is formalized in the following definition.

Definition 2.10. For any $\pi \in \Pi$, we say that $\alpha^{\pi}=\left(\alpha_{i}^{\pi}\right)_{i \in N}$ constitutes a Bayes-Nash equilibrium if for all $i \in N, s^{\prime} \in S^{\pi}$, and $a_{i} \in A_{i}$, it holds that

$$
\begin{aligned}
\sum_{\omega \in \Omega} \lambda_{i}^{s^{\prime}}(\omega) & \sum_{s \in S^{\pi}: s_{i}=s_{i}^{\prime}} \frac{\pi\left(\left(s_{i}^{\prime}, s_{-i}\right) \mid \omega\right)}{\pi_{i}\left(s_{i}^{\prime} \mid \omega\right)} u_{i}\left(z^{k}\left(\alpha_{i}^{\pi}\left(s_{i}^{\prime}\right), \alpha_{-i}^{\pi}\left(s_{-i}\right)\right), \omega\right) \\
& \geq \sum_{\omega \in \Omega} \lambda_{i}^{s^{\prime}}(\omega) \sum_{s \in S^{\pi}: s_{i}=s_{i}^{\prime}} \frac{\pi\left(\left(s_{i}^{\prime}, s_{-i}\right) \mid \omega\right)}{\pi_{i}\left(s_{i}^{\prime} \mid \omega\right)} u_{i}\left(z^{k}\left(a_{i}, \alpha_{-i}^{\pi}\left(s_{-i}\right)\right), \omega\right) .
\end{aligned}
$$

Given a signal realization and other Receivers' sincere actions, (2.13) requires that a Receiver not be able to profitably deviate from $\alpha_{i}^{\pi}\left(s_{i}\right)$. One can easily observe that if a signal $s$ is sent under which no agents are pivotal, there is no difference in the expected utility from voting for $x$ and $y$ for any Receiver. Thus, an investigation of the BNE constraint (2.13) targets signals in which some agent is pivotal. We therefore define, for each $i \in N, \pi \in \Pi$, and $m \in S_{i}^{\pi}$ the set

$$
P^{i, m}(\pi)=\left\{s \in S^{\pi}: s_{i}=m \text { and } i \text { is pivotal }\right\}
$$

We start by simplifying the BNE constraint (2.13) by means of these sets.

Lemma 2.11. Let $\lambda^{0} \in \Delta^{\circ}(\Omega)$ and $\pi \in \Pi$. Then $\alpha^{\pi}=\left(\alpha_{i}^{\pi}\right)_{i \in N}$ is a BNE if and only if for all $i \in N, m \in M_{i}^{x}(\pi)$, and $m^{\prime} \in M_{i}^{y}(\pi)$ it holds that

$$
\begin{aligned}
& \lambda^{0}(X) \sum_{s \in P^{i, m}(\pi)} \pi(s \mid X) \geq \lambda^{0}(Y) \sum_{s \in P^{i, m}(\pi)} \pi(s \mid Y), \\
& \lambda^{0}(Y) \sum_{s \in P^{i, m^{\prime}}(\pi)} \pi(s \mid Y) \geq \lambda^{0}(X) \sum_{s \in P^{i, m^{\prime}}(\pi)} \pi(s \mid X) .
\end{aligned}
$$

Because of the swing voter's curse, sincere voting cannot be an equilibrium under the optimal private communication strategy $\pi^{*}$ if $k \leq n-1$ : upon observing $x$, Receivers are pivotal with probability 1 if the state is $Y$ and not pivotal if the state is $X$. Hence, voting for $y$ is optimal. However, sincere voting is a BNE under $\pi^{*}$ if $z^{k}$ is the unanimity voting rule, i.e., if $k=n$. 


\begin{tabular}{c|cc}
$\pi^{*}$ & $\omega=X$ & $\omega=Y$ \\
\hline$(x, x, x, x, x)$ & 1 & 0 \\
$(x, x, x, y, y)$ & 0 & $\frac{1}{12}$ \\
$(x, x, y, y, x)$ & 0 & $\frac{1}{12}$ \\
$(x, y, y, x, x)$ & 0 & $\frac{1}{12}$ \\
$(y, y, x, x, x)$ & 0 & $\frac{1}{12}$ \\
$(y, x, y, x, x)$ & 0 & $\frac{1}{12}$ \\
$(y, x, x, y, x)$ & 0 & $\frac{1}{12}$ \\
$(y, x, x, x, y)$ & 0 & $\frac{1}{12}$ \\
$(x, y, x, y, x)$ & 0 & $\frac{1}{12}$ \\
$(x, x, y, x, y)$ & 0 & $\frac{1}{12}$ \\
$(x, y, x, x, y)$ & 0 & $\frac{1}{12}$ \\
$(y, y, y, y, y)$ & 0 & $\frac{1}{6}$
\end{tabular}

Table 2.1: Communication strategy $\pi^{*}$.

\begin{tabular}{c|cc}
$\pi$ & $\omega=X$ & $\omega=Y$ \\
\hline$(x, x, x, x, x)$ & 0 & 0 \\
$(x, x, x, y, y)$ & $\frac{1}{10}$ & $\frac{1}{20}$ \\
$(x, x, y, y, x)$ & $\frac{1}{10}$ & $\frac{1}{20}$ \\
$(x, y, y, x, x)$ & $\frac{1}{10}$ & $\frac{1}{20}$ \\
$(y, y, x, x, x)$ & $\frac{1}{10}$ & $\frac{1}{20}$ \\
$(y, x, y, x, x)$ & $\frac{1}{10}$ & $\frac{1}{20}$ \\
$(y, x, x, y, x)$ & $\frac{1}{10}$ & $\frac{1}{20}$ \\
$(y, x, x, x, y)$ & $\frac{1}{10}$ & $\frac{1}{20}$ \\
$(x, y, x, y, x)$ & $\frac{1}{10}$ & $\frac{1}{20}$ \\
$(x, x, y, x, y)$ & $\frac{1}{10}$ & $\frac{1}{20}$ \\
$(x, y, x, x, y)$ & $\frac{1}{10}$ & $\frac{1}{20}$ \\
$(y, y, y, y, y)$ & 0 & $\frac{1}{2}$
\end{tabular}

Table 2.2: Communication strategy $\pi$.

Proposition 2.12. Let $\lambda^{0} \in \Delta^{\circ}(\Omega)$ and let $\pi^{*} \in \Pi$ be the optimal private communication strategy in Theorem 2.8. Then $\alpha^{\pi^{*}}$ is a BNE if and only if $k=n$.

Any public communication strategy implements sincere voting as BNE: in this case no agent is ever pivotal and the two constraints in Lemma 2.11 are trivially satisfied. Proposition 2.12 is based on the fact that $\pi^{*}$ is a public communication strategy if and only if $k=n$.

Suppose $\pi^{*}$ from Theorem 2.8 is being implemented and Receiver $i$ observes $x$. Then it is in his best interest to vote $y$ as we have seen in Example 2.9. Given the constraints in Lemma 2.11, there are two intuitive ways to adjust $\pi^{*}$ which ensure sincere voting be an equilibrium: following the observation $x$ one either needs that the probability of $i$ being pivotal is higher if the state is $X$ (at $\pi^{*}$ this probability is 0 ) or lower if the state is $Y$ (at $\pi^{*}$ this probability is 1). We illustrate both approaches in the following example.

Example 2.13. Let $n=5, k=3$, and $\lambda^{0}(X)=1 / 3$. The optimal private communication strategy $\pi^{*}$ is given in Table 2.1 and a communication strategy $\pi$ with higher probability of being pivotal if the state is $X$ is given in Table 2.2. Note that $\pi$ is chosen such that $r_{3}$ is as high as possible, subject to the constraints imposed by straightforwardness (2.4) and BNE constraints (2.14) and (2.15). For any $i \in N$, we have $\lambda_{i}^{\mathbf{x}}(X)=1 / 2$, so that $\alpha_{i}^{\pi}(x)=x$. A simple calculation shows that upon receiving $x$ Receivers are indifferent between following their recommendation and deviating. That is, (2.14) holds with equality. Moreover, agents are never pivotal upon observing $y$, so (2.15) is trivially satisfied. Therefore, $\alpha^{\pi}$ is a BNE. 


\begin{tabular}{c|cc}
$\pi^{\prime}$ & $\omega=X$ & $\omega=Y$ \\
\hline$(x, x, x, x, x)$ & 1 & 0 \\
$(x, x, x, x, y)$ & 0 & $\frac{1}{8}$ \\
$(x, x, x, y, x)$ & 0 & $\frac{1}{8}$ \\
$(x, x, y, x, y)$ & 0 & $\frac{1}{8}$ \\
$(x, y, x, x, y)$ & 0 & $\frac{1}{8}$ \\
$(y, x, x, x, x)$ & 0 & $\frac{1}{8}$ \\
$(y, y, y, y, y)$ & 0 & $\frac{3}{8}$
\end{tabular}

Table 2.3: Communication strategy $\pi^{\prime}$.

However, $\pi$ does not improve upon $\pi^{* \mathrm{p}}$ :

$$
V^{\pi}\left(\frac{1}{3}\right)=\frac{1}{3} \sum_{s \in Z^{x}(\pi)} \pi(s \mid X)+\frac{2}{3} \sum_{s \in Z^{x}(\pi)} \pi(s \mid Y)=\frac{1}{3} \cdot 1+\frac{2}{3} \cdot \frac{1}{2}=\frac{2}{3}=V^{* \mathrm{p}}\left(\frac{1}{3}\right) .
$$

Now consider communication strategy $\pi^{\prime}$ in Table 2.3 where $x$ is sent to $k+1=4$ agents rather than a minimal winning coalition consisting of $k=3$ agents if the state is $Y$. Clearly, $P^{i, x}(\pi)=P^{i, y}(\pi)=\emptyset$, that is, an agent's probability of being pivotal is always 0. Thus, (2.14) and (2.15) hold with equality, so that $\alpha^{\pi^{\prime}}$ is a BNE. For all $i \in N$, we have $\lambda_{i}^{\mathbf{x}}(X)=1 / 2$ and $\lambda_{i}^{\mathbf{y}}(X)=0$. So, $\pi^{\prime}$ is straightforward as well. Moreover,

$$
V^{\pi^{\prime}}\left(\frac{1}{3}\right)=\frac{1}{3} \sum_{s \in Z^{x}\left(\pi^{\prime}\right)} \pi^{\prime}(s \mid X)+\frac{2}{3} \sum_{s \in Z^{x}\left(\pi^{\prime}\right)} \pi^{\prime}(s \mid Y)=\frac{1}{3} \cdot 1+\frac{2}{3} \cdot \frac{5}{8}=\frac{3}{4}>\frac{2}{3}=V^{* \mathrm{p}}\left(\frac{1}{3}\right),
$$

that is, $\pi^{\prime}$ improves upon $\pi^{* \mathrm{p}}$.

\subsection{Communication with Equilibrium Constraints}

We are not interested in just any BNE, but in a BNE under which Receivers vote sincerely. In Proposition 2.12 we have characterized an optimal communication strategy that implements sincere voting as BNE for the case $k=n$. In this section we will, therefore, focus on $k \leq n-1$. Let $\Pi^{\mathrm{e}}$ be the set of all communication strategies under which sincere voting constitutes a BNE, i.e., $\Pi^{\mathrm{e}}=\left\{\pi \in \Pi \mid \alpha^{\pi}\right.$ is a BNE $\}$. The sender's problem is to find $\pi \in \Pi^{\mathrm{e}}$ which maximizes her expected utility. That is,

$$
V^{* \mathrm{e}}\left(\lambda^{0}\right)=\sup _{\pi \in \Pi^{\mathrm{e}}} V^{\pi}\left(\lambda^{0}\right)
$$

We first show that even under the additional BNE constraints (2.14) and (2.15) we can still restrict attention to straightforward and anonymous communication strategies. Let $\Pi^{\text {sae }}$ denote the set of communication strategies in $\Pi^{\mathrm{e}}$ that are straightforward and anonymous. 
Lemma 2.14. Let $\lambda^{0} \in \Delta^{\circ}(\Omega)$ and $\hat{\pi} \in \Pi^{\mathrm{e}}$. Then there is $\pi \in \Pi^{\text {sae }}$ with $V^{\pi}\left(\lambda^{0}\right) \geq V^{\hat{\pi}}\left(\lambda^{0}\right)$.

Note that even though we now include the BNE requirement in showing straightforwardness, it is not equivalent to the straightforwardness result in Chan et al. (2019). In particular, we restrict attention to anonymous communication strategies as well as straightforward ones.

Lemma 2.14 allows us to express the BNE constraints of Lemma 2.11 by means of the parameters $q$ and $r$. In particular, they are equivalent to

$$
\begin{gathered}
\lambda^{0}(X) q_{k} \geq \lambda^{0}(Y) r_{k}, \\
\lambda^{0}(Y) r_{k-1} \geq \lambda^{0}(X) q_{k-1} .
\end{gathered}
$$

A trivial way to satisfy these constraints is to choose $q_{k-1}=q_{k}=r_{k}=0$. The next lemma shows that this can be done without loss of generality.

Lemma 2.15. Let $\lambda^{0} \in \Delta^{\circ}(\Omega), \hat{\pi} \in \Pi^{\mathrm{sae}}$, and $k \leq n-1$. Then there exists $\pi \in \Pi^{\mathrm{sae}}$ with representation $(q, r)$ such that $q_{k-1}=q_{k}=r_{k}=0$ and $V^{\pi}\left(\lambda^{0}\right) \geq V^{\hat{\pi}}\left(\lambda^{0}\right)$.

Lemma 2.15 is in stark contrast to Theorem 2.8: when no BNE constraint is present, it is in the sender's best interest to make Receivers pivotal upon observing $x$ if the state is $Y$, i.e., $r_{k}>0 .^{8}$ We show next how the problem can be further simplified.

Lemma 2.16. Let $\lambda^{0} \in \Delta^{\circ}(\Omega), \hat{\pi} \in \Pi^{\text {sae }}$, and $k \leq n-1$. Then there exists $\pi \in \Pi^{\text {sae }}$ with representation $(q, r)$ such that $q_{n}=1, r_{\ell}=0$ for all $\ell \neq 0, k+1$, and $V^{\pi}\left(\lambda^{0}\right) \geq V^{\hat{\pi}}\left(\lambda^{0}\right)$.

When choosing $q$ and $r$ as suggested in Lemma 2.16, constraints (2.16) and (2.17) are clearly satisfied. Thus, the optimization problem that the sender faces under the additional BNE constraint is very similar to the one in Proposition 2.7.

Proposition 2.17. Let $\lambda^{0} \in \Delta^{\circ}(\Omega)$ and $k \leq n-1$. Then $V^{* \mathrm{e}}\left(\lambda^{0}\right)$ is the solution to

$$
\begin{aligned}
\max _{r_{0}, r_{k+1}} \lambda^{0}(X)+\lambda^{0}(Y) r_{k+1} & \\
\text { s.t. } r_{0} & \geq 0, \\
r_{k+1} & \geq 0, \\
r_{0}+r_{k+1} & =1, \\
\lambda^{0}(X)-\lambda^{0}(Y) \frac{k+1}{n} r_{k+1} & \geq 0 .
\end{aligned}
$$

Interestingly, Proposition 2.17 differs from Proposition 2.7 only in that the BNE constraint shifts weight from $r_{k}$ to $r_{k+1}$. As the objective function is continuous and all constraints are weak inequalities, and because of Lemma 2.14, for any $\lambda^{0} \in \Delta^{\circ}(\Omega)$

$$
V^{* \mathrm{e}}\left(\lambda^{0}\right)=\max _{\pi \in \Pi^{\mathrm{sae}}} V^{\pi}\left(\lambda^{0}\right)
$$

\footnotetext{
${ }^{8}$ We should mention that Lemma 2.15 is not true for $k=n$, as in that case it is optimal for the sender to make all Receivers observe $x$ if the state is $X$, i.e., $q_{k}=q_{n}=1$.
} 
so there is $\pi \in \Pi^{\text {sae }}$ that achieves the optimal value. Such an optimal communication strategy is given in Theorem 2.18.

Theorem 2.18. Let $\lambda^{0} \in \Delta^{\circ}(\Omega)$. If $k \leq n-1$, then the communication strategy $\pi^{* \mathrm{e}} \in \Pi^{\text {sae }}$ with representation $\left(q^{* \mathrm{e}}, r^{* \mathrm{e}}\right)$ given by

$$
\left(q_{n}^{* \mathrm{e}} ; r_{0}^{* \mathrm{e}}, r_{k+1}^{* \mathrm{e}}\right)= \begin{cases}(1 ; 0,1) & \text { if } \lambda^{0}(X) \geq \frac{k+1}{n+k+1} \\ \left(1 ; 1-\frac{\lambda^{0}(X)}{\lambda^{0}(Y)} \frac{n}{k+1}, \frac{\lambda^{0}(X)}{\lambda^{0}(Y)} \frac{n}{k+1}\right) & \text { if } \lambda^{0}(X)<\frac{k+1}{n+k+1}\end{cases}
$$

is optimal at $\lambda^{0}$ in $\Pi^{\mathrm{e}}$. In particular, the optimal value function is given by

$$
V^{* \mathrm{e}}\left(\lambda^{0}\right)=\min \left\{\frac{n+k+1}{k+1} \lambda^{0}(X), 1\right\} .
$$

Theorem 2.18 covers the case that voting is not unanimous. Lemma 2.16 establishes that there is an optimal communication strategy $\pi^{* \mathrm{e}}$ such that $q_{n}^{* \mathrm{e}}=1$. If $\lambda^{0}(X) \geq(k+1) /(n+k+1)$, then it is optimal to send $x$ with probability 1 to a coalition of $k+1$ Receivers if the state is $Y$. So, Receivers are not pivotal in either state and $x$ is implemented with probability 1 . If $\lambda^{0}(X)<(k+1) /(n+k+1)$, then $r_{0}^{* \mathrm{e}}>0$. As there is a positive probability that $x$ is not implemented, the value of the optimal communication strategy is strictly less than 1 .

If $k \geq n-1$, Proposition 2.12 and Theorem 2.18 together imply that a public signal is optimal. Yet, if $k=n-1$, there is another, very different, optimal communication strategy: in Example 2.13 we presented $\pi$ which had the property that agents were pivotal upon observing $x$ in either state, and we showed that this strategy obtains the same value as the optimal public communication strategy $\pi^{* \mathrm{p}}$. The fact that $\pi^{* \mathrm{p}}$ is optimal under the BNE constraint if $k=n-1$ motivates the following corollary.

Corollary 2.19. Let $k=n-1$ and $\lambda^{0} \in \Delta^{\circ}(\Omega)$. The communication strategy represented by $q_{k}=1, r_{k}=\min \left\{\lambda^{0}(X) / \lambda^{0}(Y), 1\right\}$, and $r_{0}=1-r_{k}$ is optimal.

In order to prove the corollary, one only needs to verify that the straightforwardness constraints 2.4 and 2.5 and the BNE constraints 2.16 and 2.17 are satisfied and that this communication strategy performs as well as $\pi^{* \mathrm{p}}$. The corollary highlights a fundamentally different way to achieve the optimal value in this setting.

If $k \leq n-1$, designing the communication strategy so that sincere behavior constitutes a BNE is not without a cost for the sender, namely losing some probability of implementing her preferred outcome.

Corollary 2.20. Let $\lambda^{0} \in \Delta^{\circ}(\Omega)$.

(i) If $k \leq n-1$ then $V^{* \mathrm{p}}\left(\lambda^{0}\right) \leq V^{* \mathrm{e}}\left(\lambda^{0}\right) \leq V^{*}\left(\lambda^{0}\right) \leq \frac{k+1}{k} \frac{n+k}{n+k+1} V^{* \mathrm{e}}\left(\lambda^{0}\right)$.

(ii) If $k=n$ then $V^{*}\left(\lambda^{0}\right)=V^{* \mathrm{p}}\left(\lambda^{0}\right)=V^{* \mathrm{e}}\left(\lambda^{0}\right)$. 


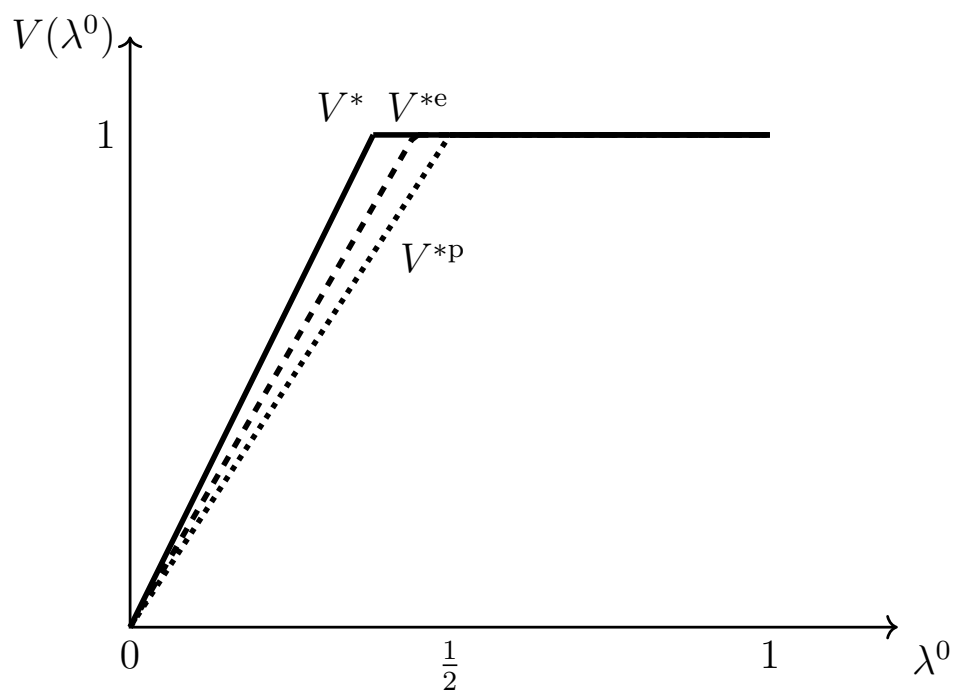

Figure 2.1: Values of $\pi^{*}, \pi^{* \mathrm{e}}$ and $\pi^{* \mathrm{p}}$ for $n=5$ and $k=3$.

In Figure 2.1, we plot the value functions $V^{*}, V^{* \mathrm{e}}$ and $V^{* \mathrm{p}}$ for $n=5$ and $k=3$. The sender's expected utility is highest when sincere voting can be taken for granted, which is given by the straight line in Figure 2.1. Under the BNE constraint she cannot prevent a decrease in the probability of implementing her preferred outcome and ends up with a lower expected utility, i.e., $V^{*}\left(\lambda^{0}\right)>V^{* e}\left(\lambda^{0}\right)$ for $\lambda^{0}(X)<(k+1) /(n+k+1)$.

It is easy to see that the value of an optimal communication strategy which implements sincere voting as BNE is decreasing in the quota.

Corollary 2.21. Let $\lambda^{0} \in \Delta^{\circ}(\Omega)$. The optimal value $V^{* \mathrm{e}}\left(\lambda^{0}\right)$ is weakly decreasing in $k$.

This result is intuitive, since persuasion becomes more difficult for the sender as the number of receivers that have to be convinced increases. In particular, the value of the optimal communication strategy which implements sincere voting as BNE decreases with $k$ and equals the value of the optimal public communication strategy if $k \geq n-1$. In contrast, if we fix the quota, then the sender can implement $x$ with probability 1 when the number of Receivers tends to infinity, i.e., $\lim _{n \rightarrow \infty} V^{* \mathrm{e}}\left(\lambda^{0}\right)=1$. Finally, if the quota is a fixed ratio of the number of voters as in case of majority voting, Theorem 2.18 reveals that the cost of implementing sincere voting as BNE decreases as the population increases. In particular, if $k=\lceil p n\rceil$ for some $p \in(0,1]$, one has, for all $\lambda^{0} \in \Delta^{\circ}(\Omega)$,

$$
\lim _{n \rightarrow \infty} V^{* \mathrm{e}}\left(\lambda^{0}\right)=\lim _{n \rightarrow \infty} V^{*}\left(\lambda^{0}\right)= \begin{cases}1 & \text { if } \lambda^{0}(X) \geq \frac{p}{1+p} \\ \frac{1+p}{p} \lambda^{0}(X) & \text { if } \lambda^{0}(X)<\frac{p}{1+p}\end{cases}
$$




\subsection{Conclusion}

This paper investigates Bayesian persuasion where prior to a vote, a sender attempts to persuade the receivers to vote for her favorite outcome. Motivated by social media that allow for targeted communication with individual receivers, we allow the sender to use private messages that may be correlated. While it is indeed beneficial for the sender to employ a private communication strategy with correlated messages rather than a public communication strategy, this might lead to the swing voter's curse, that is, situations in which sincere voting is not an equilibrium. We show that the most efficient way for the sender to ensure that sincere voting be in the best interest of each Receiver is to send signals after which nobody is pivotal. That is, instead of persuading minimal winning coalitions, the sender should target slightly larger coalitions. This comes at a cost in terms of success probability, but this cost decreases as the population increases.

\subsection{Appendix}

\subsubsection{Straightforwardness}

We provide the formal versions and the proofs of the results we mention in Section 2.3.1.

Lemma 2.22. Let $\lambda^{0}(X) \in(0,1 / 2)$. Then $\pi \in \Pi^{\mathrm{s}}$ if and only if $\pi$ satisfies (2.4).

Proof. It is sufficient to show that (2.4) implies (2.5). So, let (2.4) be satisfied. Then

$$
\begin{aligned}
\lambda^{0}(X) \pi_{i}(y \mid X) & =\lambda^{0}(X)\left(1-\pi_{i}(x \mid X)\right) \\
& \leq \lambda^{0}(X)-\lambda^{0}(Y) \pi_{i}(x \mid Y) \\
& =\lambda^{0}(X)-\lambda^{0}(Y)+\lambda^{0}(Y) \pi_{i}(y \mid Y) \\
& <\lambda^{0}(Y) \pi_{i}(y \mid Y),
\end{aligned}
$$

where the first inequality follows from (2.4) and the last inequality holds since $\lambda^{0}(X)<1 / 2<$ $\lambda^{0}(Y)$. So, $\pi$ satisfies (2.5). Hence, $\pi$ is straightforward.

Lemma 2.23. Let $\lambda^{0} \in \Delta^{\circ}(\Omega)$ and $\hat{\pi} \in \Pi$. Then there is $\pi \in \Pi^{\mathrm{s}}$ with $V^{\pi}\left(\lambda^{0}\right) \geq V^{\hat{\pi}}\left(\lambda^{0}\right)$.

Proof. If $\lambda^{0}(X) \geq 1 / 2$, let $\pi \in \Pi^{\mathrm{s}}$ be defined as $\pi(x \mid \omega)=1$ for all $\omega \in \Omega$ and observe that $V^{\pi}\left(\lambda^{0}\right)=1 \geq V^{\hat{\pi}}\left(\lambda^{0}\right)$. Let $\lambda^{0}(X) \in(0,1 / 2)$. For each action profile $a \in A$, define $S^{a}(\hat{\pi})=\left\{s \in S^{\hat{\pi}} \mid \alpha^{\hat{\pi}}(s)=a\right\}$, which is the set of signals in $S^{\hat{\pi}}$ that lead to action profile $a$ under sincere voting. Note that for $a \neq b$, we have $S^{a}(\hat{\pi}) \cap S^{b}(\hat{\pi})=\emptyset$. Define a communication strategy $\pi \in \Pi$ by $\pi(a \mid \omega)=\sum_{s \in S^{a}(\hat{\pi})} \hat{\pi}(s \mid \omega)$ for each $\omega \in \Omega$ and $a \in A$, and note that $S_{i}^{\pi} \subseteq A_{i}$ for all $i \in N$. Fix $i \in N$. Then, for any $\omega \in \Omega$ and $a_{i} \in S_{i}^{\pi}$,

$$
\pi_{i}\left(a_{i} \mid \omega\right)=\sum_{t \in S^{\pi}: t_{i}=a_{i}} \pi(t \mid \omega)=\sum_{t \in S^{\pi}: t_{i}=a_{i}} \sum_{s \in S^{t}(\hat{\pi})} \hat{\pi}(s \mid \omega) .
$$


Recall that for each $i \in N$ we have $M_{i}^{x}(\hat{\pi})=\left\{m \in S_{i}^{\hat{\pi}} \mid \alpha_{i}^{\hat{\pi}}(m)=x\right\}$, which is the set of messages after which $i$ chooses action $x$ under sincere voting. Since $\lambda^{0}(X) \in(0,1 / 2)$, by Lemma 2.22 it is sufficient to show that $\pi$ satisfies (2.4).

Claim: It holds that

$$
\bigcup_{t \in S^{\pi}: t_{i}=x} S^{t}(\hat{\pi})=\bigcup_{m \in M_{i}^{x}(\hat{\pi})}\left\{t^{\prime} \in S^{\hat{\pi}}: t_{i}^{\prime}=m\right\}
$$

and both unions are over disjoint sets.

Proof. Let $s \in \bigcup_{t \in S^{\pi}: t_{i}=x} S^{t}(\hat{\pi})$. Then, there exists $t \in S^{\pi}$ with $t_{i}=x$ such that $s \in$ $S^{t}(\hat{\pi})$. Thus, by the definition of $S^{t}(\pi), \alpha_{i}^{\hat{\pi}}\left(s_{i}\right)=x$, so that $s_{i} \in M_{i}^{x}(\hat{\pi})$. In particular, $s \in\left\{t^{\prime} \in S^{\hat{\pi}}: t_{i}^{\prime}=s_{i}\right\} \subseteq \bigcup_{m \in M_{i}^{x}(\hat{\pi})}\left\{t^{\prime} \in S^{\hat{\pi}}: t_{i}^{\prime}=m\right\}$. For the converse, suppose $s \in$ $\bigcup_{m \in M_{i}^{x}(\hat{\pi})}\left\{t^{\prime} \in S^{\hat{\pi}}: t_{i}^{\prime}=m\right\}$. Then there exists $m \in M_{i}^{x}(\hat{\pi})$ with $s_{i}=m$. Let $t=\alpha^{\hat{\pi}}(s) \in A$ and observe that $t_{i}=\alpha_{i}^{\hat{\pi}}(m)=x$. Since by construction $s \in S^{t}(\hat{\pi})$ and $t \in S^{\pi}$, it holds that $s \in \bigcup_{t \in S^{\pi}: t_{i}=x} S^{t}(\hat{\pi})$.

As noted before, for any $a, b \in S^{\pi}$ with $a \neq b$ we have $S^{a}(\hat{\pi}) \cap S^{b}(\hat{\pi})=\emptyset$. Moreover, for any $m, m^{\prime} \in M_{i}^{x}(\hat{\pi})$ with $m \neq m^{\prime}$, we have $\left\{t^{\prime} \in S^{\hat{\pi}}: t_{i}^{\prime}=m\right\} \cap\left\{t^{\prime} \in S^{\hat{\pi}}: t_{i}^{\prime}=m^{\prime}\right\}=\emptyset$. Thus, both unions are over disjoint sets. This proves the claim.

Let $M_{i}^{x}(\hat{\pi})=\left\{m_{1}, \ldots, m_{L}\right\}$ for some $L \geq 1$. For each $\ell \in\{1, \ldots, L\}, m_{\ell} \in M_{i}^{x}(\hat{\pi})$, and $s \in S^{\hat{\pi}}$ with $s_{i}=m_{\ell}$, let

$$
\begin{aligned}
c_{\ell} & =\sum_{t \in S^{\hat{\pi}}: t_{i}=m_{\ell}} \hat{\pi}(t \mid X) \lambda^{0}(X), \\
d_{\ell} & =\sum_{t \in S^{\hat{\pi}}: t_{i}=m_{\ell}} \hat{\pi}(t \mid X) \lambda^{0}(X)+\sum_{t \in S^{\hat{\pi}}: t_{i}=m_{\ell}} \hat{\pi}(t \mid Y) \lambda^{0}(Y),
\end{aligned}
$$

and note that $c_{\ell} / d_{\ell}=\lambda_{i}^{s}(X) \geq 1 / 2$ since $\alpha_{i}^{\hat{\pi}}\left(m_{\ell}\right)=x$. Thus, it holds that $d_{\ell} \leq 2 c_{\ell}$. Let $t \in S^{\pi}$ be such that $t_{i}=x$. We have that

$$
\begin{aligned}
\lambda_{i}^{t}(X) & =\frac{\pi_{i}(x \mid X) \lambda^{0}(X)}{\pi_{i}(x \mid X) \lambda^{0}(X)+\pi_{i}(x \mid Y) \lambda^{0}(Y)} \\
& =\frac{\sum_{t \in S^{\pi}: t_{i}=x} \sum_{s \in S^{t}(\hat{\pi})} \hat{\pi}(s \mid X) \lambda^{0}(X)}{\sum_{t \in S^{\pi}: t_{i}=x} \sum_{s \in S^{t}(\hat{\pi})} \hat{\pi}(s \mid X) \lambda^{0}(X)+\sum_{t \in S^{\pi}: t_{i}=x} \sum_{s \in S^{t}(\hat{\pi})} \hat{\pi}(s \mid Y) \lambda^{0}(Y)} \\
& \stackrel{\sum_{m \in M_{i}^{x}(\hat{\pi})} \sum_{t \in S^{\hat{\pi}}: t_{i}=m} \hat{\pi}(t \mid X) \lambda^{0}(X)}{=} \frac{\sum_{m \in M_{i}^{x}(\hat{\pi})} \sum_{t \in S^{\hat{\pi}}: t_{i}=m} \hat{\pi}(t \mid X) \lambda^{0}(X)+\sum_{m \in M_{i}^{x}(\hat{\pi})} \sum_{t \in S^{\hat{\pi}}: t_{i}=m} \hat{\pi}(t \mid Y) \lambda^{0}(Y)}{\sum_{L}+\cdots+c_{L}} \\
& =\frac{c_{1}+\cdots+c_{L}}{d_{1}+\cdots+d_{L}} \geq \frac{c_{1}+\cdots}{2\left(c_{1}+\cdots+c_{L}\right)}=\frac{1}{2} .
\end{aligned}
$$

Thus, for any $m \in M_{i}^{x}(\hat{\pi})$, we have $\alpha_{i}^{\pi}(x)=\alpha_{i}^{\hat{\pi}}(m)=x$. Hence, $\pi$ satisfies (2.4).

What remains to be shown is that $\pi$ has at least the same value as $\hat{\pi}$. By definition of $Z^{x}(\pi)$ it holds that

$$
\sum_{a \in Z^{x}(\pi)} \pi(a \mid \omega)=\sum_{a \in Z^{x}(\pi)} \sum_{s \in S^{a}(\hat{\pi})} \hat{\pi}(s \mid \omega)=\sum_{s \in Z^{x}(\hat{\pi})} \hat{\pi}(s \mid \omega) .
$$


Thus, we have

$$
\begin{aligned}
V^{\pi}\left(\lambda^{0}\right) & =\lambda^{0}(X) \sum_{s \in Z^{x}(\pi)} \pi(s \mid X)+\lambda^{0}(Y) \sum_{s \in Z^{x}(\pi)} \pi(s \mid Y) \\
& =\lambda^{0}(X) \sum_{s \in Z^{x}(\hat{\pi})} \hat{\pi}(s \mid X)+\lambda^{0}(Y) \sum_{s \in Z^{x}(\hat{\pi})} \hat{\pi}(s \mid Y) \\
& =V^{\hat{\pi}}\left(\lambda^{0}\right)
\end{aligned}
$$

as required.

\subsubsection{Proofs}

Proof of Lemma 2.4. If $\lambda^{0}(X) \geq 1 / 2$, then let $\pi \in \Pi^{\mathrm{s}}$ be defined as $\pi(x \mid \omega)=1$ for all $\omega \in$ $\Omega$ and observe that $V^{\pi}\left(\lambda^{0}\right)=1 \geq V^{\hat{\pi}}\left(\lambda^{0}\right)$. Let $\lambda^{0}(X)<1 / 2$. By Lemma 2.23, we can assume without loss of generality that $\hat{\pi} \in \Pi^{\mathrm{s}}$. Thus, by Lemma $2.22, \lambda^{0}(X) \hat{\pi}_{i}(x \mid X) \geq \lambda^{0}(Y) \hat{\pi}_{i}(x \mid Y)$ for all $i \in N$. Let $\pi \in \Pi^{\mathrm{s}}$ be the communication strategy such that for all $i \in N$ and $s_{i} \in S_{i}$, $\pi_{i}\left(s_{i} \mid Y\right)=\hat{\pi}_{i}\left(s_{i} \mid Y\right)$ and $\pi_{i}(x \mid X)=1$. That is, signal probabilities in state $Y$ remain the same, but $\pi$ sends $x$ to every Receiver with probability 1 if the state is $X$. Then

$$
\lambda^{0}(X) \pi_{i}(x \mid X) \geq \lambda^{0}(X) \hat{\pi}_{i}(x \mid X) \geq \lambda^{0}(Y) \hat{\pi}_{i}(x \mid Y)=\lambda^{0}(Y) \pi_{i}(x \mid Y),
$$

where the second inequality holds since $\hat{\pi} \in \Pi^{\mathrm{s}}$. By Lemma 2.22, $\pi$ is straightforward. Finally,

$$
\begin{aligned}
V^{\pi}\left(\lambda^{0}\right) & =\lambda^{0}(X)+\lambda^{0}(Y) \sum_{s \in Z^{x}(\pi)} \pi(s \mid Y) \geq \lambda^{0}(X) \sum_{s \in Z^{x}(\hat{\pi})} \hat{\pi}(s \mid X)+\lambda^{0}(Y) \sum_{s \in Z^{x}(\hat{\pi})} \hat{\pi}(s \mid Y) \\
& =V^{\hat{\pi}}\left(\lambda^{0}\right)
\end{aligned}
$$

which completes the proof.

Proof of Lemma 2.5. If $\lambda^{0}(X) \geq 1 / 2$, then let $\pi \in \Pi^{\text {sa }}$ be defined as $\pi(x \mid \omega)=1$ for all $\omega \in \Omega$ and observe that $V^{\pi}\left(\lambda^{0}\right)=1 \geq V^{\hat{\pi}}\left(\lambda^{0}\right)$. Let $\lambda^{0}(X)<1 / 2$. By Lemma 2.23, assume without loss of generality that $\hat{\pi}$ is straightforward. For each $\omega \in \Omega, s \in S$, and $b \in B$ define $\pi^{b}$ by $\pi^{b}(s \mid \omega)=\hat{\pi}\left(s^{b} \mid \omega\right)$, so that $S^{\pi^{b}}=\left\{s^{b^{-1}} \mid s \in S^{\hat{\pi}}\right\}$. Let $\pi \in \Pi$ be defined by

$$
\pi(s \mid \omega)=\frac{1}{n !} \sum_{b \in B} \pi^{b}(s \mid \omega), \quad \omega \in \Omega, s \in S .
$$

Clearly, $\pi$ is anonymous. We next show that $\pi$ is straightforward. Fix $i \in N$. Then

$$
\begin{aligned}
\pi_{i}(x \mid X) & =\sum_{s \in S^{\pi}: s_{i}=x} \pi(s \mid X)=\sum_{s \in S^{\pi}: s_{i}=x} \frac{1}{n !} \sum_{b \in B} \pi^{b}(s \mid X) \\
& =\frac{1}{n !} \sum_{b \in B} \sum_{s \in S^{\pi}: s_{i}=x} \hat{\pi}\left(s^{b} \mid X\right)=\frac{1}{n !} \sum_{b \in B} \sum_{s \in S^{\pi}: s_{b(i)}=x} \hat{\pi}(s \mid X) \\
& =\frac{1}{n !} \sum_{b \in B} \hat{\pi}_{b(i)}(x \mid X) .
\end{aligned}
$$


By Lemma 2.22, one has $\lambda^{0}(X) \hat{\pi}_{b(i)}(x \mid X) \geq \lambda^{0}(Y) \hat{\pi}_{b(i)}(x \mid Y)$ for all $b \in B$. Thus,

$$
\lambda^{0}(X) \pi_{i}(x \mid X)=\lambda^{0}(X) \frac{1}{n !} \sum_{b \in B} \hat{\pi}_{b(i)}(x \mid X) \geq \lambda^{0}(Y) \frac{1}{n !} \sum_{b \in B} \hat{\pi}_{b(i)}(x \mid Y)=\lambda^{0}(Y) \pi_{i}(x \mid Y) .
$$

Hence, $\pi$ satisfies (2.4) and, by Lemma 2.22, is straightforward. Finally,

$$
\begin{aligned}
V^{\pi}\left(\lambda^{0}\right) & =\lambda^{0}(X) \sum_{s \in Z^{x}(\pi)} \pi(s \mid X)+\lambda^{0}(Y) \sum_{s \in Z^{x}(\pi)} \pi(s \mid Y) \\
& =\lambda^{0}(X) \sum_{s \in Z^{x}(\pi)} \frac{1}{n !} \sum_{b \in B} \pi^{b}(s \mid X)+\lambda^{0}(Y) \sum_{s \in Z^{x}(\pi)} \frac{1}{n !} \sum_{b \in B} \pi^{b}(s \mid Y) \\
& =\lambda^{0}(X) \frac{1}{n !} \sum_{b \in B} \sum_{s \in Z^{x}\left(\pi^{b}\right)} \pi^{b}(s \mid X)+\lambda^{0}(Y) \frac{1}{n !} \sum_{b \in B} \sum_{s \in Z^{x}\left(\pi^{b}\right)} \pi^{b}(s \mid Y) \\
& =\lambda^{0}(X) \frac{1}{n !} \sum_{b \in B} \sum_{s \in Z^{x}(\hat{\pi})} \hat{\pi}(s \mid X)+\lambda^{0}(Y) \frac{1}{n !} \sum_{b \in B} \sum_{s \in Z^{x}(\hat{\pi})} \hat{\pi}(s \mid Y) \\
& =\lambda^{0}(X) \sum_{s \in Z^{x}(\hat{\pi})} \hat{\pi}(s \mid X)+\lambda^{0}(Y) \sum_{s \in Z^{x}(\hat{\pi})} \hat{\pi}(s \mid Y) \\
& =V^{\hat{\pi}}\left(\lambda^{0}\right) .
\end{aligned}
$$

where the fourth equation holds because $Z^{x}\left(\pi^{b}\right)=\left\{s^{b^{-1}} \mid s \in Z^{x}(\hat{\pi})\right\}$.

Proof of Proposition 2.7. If $\lambda^{0}(X) \geq 1 / 2$, then the solution to the linear optimization problem in Proposition 2.7 is given by $\left(r_{0}^{*}, r_{k}^{*}\right)=(0,1)$, leading to $V^{*}\left(\lambda^{0}\right)=1$ as desired. Let $\lambda^{0}(X)<1 / 2$. First note that the communication strategy $\pi$ as constructed in the proof of Lemma 2.4 satisfies anonymity if $\hat{\pi}$ is anonymous. Hence, $V^{*}\left(\lambda^{0}\right)$ can be obtained by an anonymous and straightforward communication strategy with $q_{n}=1$. We first show that it is no loss of generality to have $r_{\ell}=0$ for all $\ell \neq 0, k$. For this purpose let $\hat{\pi} \in \Pi^{\text {sa }}$ be a communication strategy with representation $(\hat{q}, \hat{r})$. Let $\pi \in \Pi^{\mathrm{sa}}$ with representation $(q, r)$ be defined as $q_{n}=1, r_{0}=\hat{r}_{0}+\cdots+\hat{r}_{k-1}$ and $r_{k}=\hat{r}_{k}+\cdots+\hat{r}_{n}$. Then $\sum_{s \in Z^{x}(\pi)} \pi(s \mid X)=\sum_{s \in Z^{x}(\hat{\pi})} \hat{\pi}(s \mid X)$ and $\sum_{s \in Z^{x}(\pi)} \pi(s \mid Y)=\sum_{s \in Z^{x}(\hat{\pi})} \hat{\pi}(s \mid Y)$, so that $V^{\pi}\left(\lambda^{0}\right)=V^{\hat{\pi}}\left(\lambda^{0}\right)$. Therefore, the sender can restrict attention to weights $r_{0}$ and $r_{k}$ that satisfy the Constraints (2.9)-(2.11). By Lemma $2.22, \pi$ is straightforward if and only if

$$
0 \leq \lambda^{0}(X) \pi_{i}(x \mid X)-\lambda^{0}(Y) \pi_{i}(x \mid Y)=\lambda^{0}(X)-\lambda^{0}(Y) \frac{k}{n} r_{k},
$$

which is equivalent to (2.12). The objective function is, thus,

$$
\begin{aligned}
V^{\pi}\left(\lambda^{0}\right) & =\lambda^{0}(X) \sum_{s \in Z^{x}(\pi)} \pi(s \mid X)+\lambda^{0}(Y) \sum_{s \in Z^{x}(\pi)} \pi(s \mid Y)=\lambda^{0}(X) \cdot 1+\lambda^{0}(Y) r_{k} \\
& =\lambda^{0}(X)+\lambda^{0}(Y) r_{k}
\end{aligned}
$$

as required. 
Proof of Theorem 2.8. The sender's maximization problem is given in Proposition 2.7. As the objective function is increasing in $r_{k}$, Constraints (2.11) and (2.12) imply

$$
r_{k}^{*}=\min \left\{\frac{\lambda^{0}(X)}{\lambda^{0}(Y)} \frac{n}{k}, 1\right\}= \begin{cases}1 & \text { if } \lambda^{0}(X) \geq \frac{k}{n+k} \\ \frac{\lambda^{0}(X)}{\lambda^{0}(Y)} \frac{n}{k} & \text { if } \lambda^{0}(X)<\frac{k}{n+k}\end{cases}
$$

Thus, the optimal value for any $\lambda^{0} \in \Delta^{\circ}(\Omega)$ is

$$
V^{\pi^{*}}\left(\lambda^{0}\right)=\lambda^{0}(X)+\lambda^{0}(Y) r_{k}= \begin{cases}1 & \text { if } \lambda^{0}(X) \geq \frac{k}{n+k} \\ \frac{n+k}{k} \lambda^{0}(X) & \text { if } \lambda^{0}(X)<\frac{k}{n+k}\end{cases}
$$

as required.

Proof of Lemma 2.11. Let $i \in N$ be fixed. For any $s \in S^{\pi}$ it holds that

$$
\begin{gathered}
u_{i}\left(z^{k}\left(x, \alpha_{-i}^{\pi}\left(s_{-i}\right)\right), X\right)-u_{i}\left(z^{k}\left(y, \alpha_{-i}^{\pi}\left(s_{-i}\right)\right), X\right)= \begin{cases}1 & \text { if } i \text { is pivotal at } s, \\
0 & \text { if } i \text { is not pivotal at } s,\end{cases} \\
u_{i}\left(z^{k}\left(y, \alpha_{-i}^{\pi}\left(t_{-i}\right)\right), Y\right)-u_{i}\left(z^{k}\left(x, \alpha_{-i}^{\pi}\left(t_{-i}\right)\right), Y\right)= \begin{cases}1 & \text { if } i \text { is pivotal at } s, \\
0 & \text { if } i \text { is not pivotal at } s .\end{cases}
\end{gathered}
$$

For given $m \in M_{i}^{x}(\pi)$, let $s^{\prime} \in S^{\pi}$ be such that $s_{i}^{\prime}=m$ and let $c=\sum_{\omega \in \Omega} \lambda^{0}(\omega) \pi_{i}\left(s_{i}^{\prime} \mid \omega\right)$. Then

$$
\begin{aligned}
\lambda^{0}(X) & \sum_{s \in P^{i, m}(\pi)} \pi(s \mid X)-\lambda^{0}(Y) \sum_{s \in P^{i, m}(\pi)} \pi(s \mid Y) \\
= & c\left(\frac{\lambda^{0}(X) \pi_{i}\left(s_{i}^{\prime} \mid X\right)}{c} \sum_{s \in P^{i, m}(\pi)} \frac{\pi(s \mid X)}{\pi_{i}\left(s_{i}^{\prime} \mid X\right)}-\frac{\lambda^{0}(Y) \pi_{i}\left(s_{i}^{\prime} \mid Y\right)}{c} \sum_{s \in P^{i, m}(\pi)} \frac{\pi(s \mid Y)}{\pi_{i}\left(s_{i}^{\prime} \mid Y\right)}\right) \\
= & c\left(\lambda_{i}^{s^{\prime}}(X) \sum_{s \in P^{i, m}(\pi)} \frac{\pi(s \mid X)}{\pi_{i}\left(s_{i}^{\prime} \mid X\right)}\left(u_{i}\left(z^{k}\left(x, \alpha_{-i}^{\pi}\left(s_{-i}\right)\right), X\right)-u_{i}\left(z^{k}\left(y, \alpha_{-i}^{\pi}\left(s_{-i}\right)\right), X\right)\right)\right. \\
& \left.-\lambda_{i}^{s^{\prime}}(Y) \sum_{s \in P^{i, m}(\pi)} \frac{\pi(s \mid Y)}{\pi_{i}\left(s_{i}^{\prime} \mid Y\right)}\left(u_{i}\left(z^{k}\left(y, \alpha_{-i}^{\pi}\left(s_{-i}\right)\right), Y\right)\right)-u_{i}\left(z^{k}\left(x, \alpha_{-i}^{\pi}\left(s_{-i}\right)\right), Y\right)\right) \\
= & c \sum_{\omega \in \Omega} \lambda_{i}^{s^{\prime}}(\omega) \sum_{s \in S^{\pi}: s_{i}=s_{i}^{\prime}} \frac{\pi(s \mid \omega)}{\pi_{i}\left(s_{i}^{\prime} \mid \omega\right)}\left(u_{i}\left(z^{k}\left(x, \alpha_{-i}^{\pi}\left(s_{-i}\right)\right), \omega\right)-u_{i}\left(z^{k}\left(y, \alpha_{-i}^{\pi}\left(s_{-i}\right)\right), \omega\right)\right)
\end{aligned}
$$

and one shows similarly that for given $m^{\prime} \in M_{i}^{y}(\pi)$ and $t^{\prime} \in S^{\pi}$ with $t_{i}^{\prime}=m^{\prime}$

$$
\begin{aligned}
& \lambda^{0}(Y) \sum_{t \in P^{i, m^{\prime}}(\pi)} \pi(t \mid Y)-\lambda^{0}(X) \sum_{t \in P^{i, m^{\prime}}(\pi)} \pi(s \mid X) \\
& =c^{\prime} \sum_{\omega \in \Omega} \lambda_{i}^{t^{\prime}}(\omega) \sum_{t \in S^{\pi}: t_{i}=t_{i}^{\prime}} \frac{\pi(t \mid \omega)}{\pi_{i}\left(t_{i}^{\prime} \mid \omega\right)}\left(u_{i}\left(z^{k}\left(y, \alpha_{-i}^{\pi}\left(t_{-i}\right)\right), \omega\right)-u_{i}\left(z^{k}\left(x, \alpha_{-i}^{\pi}\left(t_{-i}\right)\right), \omega\right)\right),
\end{aligned}
$$

where $c^{\prime}=\sum_{\omega \in \Omega} \lambda^{0}(\omega) \pi_{i}\left(t_{i}^{\prime} \mid \omega\right)$. Thus, (2.13) holds if and only if (2.14) and (2.15) hold. 
Proof of Proposition 2.12. Let $i \in N$ and suppose first that $k \leq n-1$. Then upon receiving $x, i$ is pivotal if the state is $Y$ but not pivotal if the state is $X$. Thus, we have $\sum_{s \in P^{i, x}\left(\pi^{*}\right)} \pi^{*}(s \mid X)=0$ and $\sum_{s \in P^{i, x}\left(\pi^{*}\right)} \pi^{*}(s \mid Y)>0$. If $\alpha^{\pi^{*}}$ were a BNE at $\pi^{*},(2.14)$ would imply that: $0 \geq \lambda^{0}(Y) \sum_{s \in P^{i, x}\left(\pi^{*}\right)} \pi^{*}(s \mid Y)>0$, which is impossible.

Let $k=n$. Since $i$ is not pivotal upon observing $y,(2.15)$ is trivially satisfied, and we only need to check (2.14). By Theorem 2.8 we have $q_{n}^{*}=1$ and $r_{n}^{*}=\min \left\{\lambda^{0}(X) / \lambda^{0}(Y), 1\right\}$. Thus,

$$
\lambda^{0}(X) \sum_{s \in P^{i, x}\left(\pi^{*}\right)} \pi^{*}(s \mid X)=\lambda^{0}(X)=\lambda^{0}(Y) \frac{\lambda^{0}(X)}{\lambda^{0}(Y)} \geq \lambda^{0}(Y) r_{n}^{*}=\lambda^{0}(Y) \sum_{s \in P^{i, x}\left(\pi^{*}\right)} \pi^{*}(s \mid Y) .
$$

Hence, (2.14) is satisfied and $\alpha^{\pi^{*}}$ is a BNE.

Proof of Lemma 2.14. If $\lambda^{0}(X) \geq 1 / 2$, then consider $\pi \in \Pi^{\text {sae }}$ with representation $(q, r)$ such that $q_{n}=1$ and $r_{k+1}=1$. Observe that $V^{\pi}\left(\lambda^{0}\right)=1 \geq V^{\hat{\pi}}\left(\lambda^{0}\right)$. Let $\lambda^{0}(X)<1 / 2$. We first show that without loss of generality we can assume $\hat{\pi}$ to be anonymous. Also, without loss of generality, we assume that for all $i, j \in N$ with $i \neq j$, we have $S_{i}^{\hat{\pi}} \cap S_{j}^{\hat{\pi}}=\emptyset$, i.e., different Receivers observe different messages. Let $\pi^{\prime} \in \Pi$ be defined as

$$
\pi^{\prime}(s \mid \omega)=\frac{1}{n !} \sum_{b \in B} \hat{\pi}\left(s^{b} \mid \omega\right), \quad \omega \in \Omega, s \in S .
$$

We showed in the proof of Lemma 2.5 that $\pi^{\prime} \in \Pi^{\mathrm{a}}$ and has value greater than or equal to $\hat{\pi}$. It is therefore sufficient to show that $\alpha^{\pi^{\prime}}$ is a BNE. Let $i \in N$ and $m \in M_{i}^{x}\left(\pi^{\prime}\right)$. Let $j \in N$ be the unique Receiver such that $m \in S_{j}^{\hat{\pi}}$. Then

$$
\sum_{s \in P^{i, m}\left(\pi^{\prime}\right)} \sum_{b \in B} \hat{\pi}\left(s^{b} \mid X\right)=\sum_{s \in P^{i, m}\left(\pi^{\prime}\right)} \sum_{b \in B: b(i)=j} \hat{\pi}\left(s^{b} \mid X\right)=(n-1) ! \sum_{s \in P^{j, m}(\hat{\pi})} \hat{\pi}(s \mid X) .
$$

Therefore,

$$
\begin{aligned}
\lambda^{0}(X) \sum_{s \in P^{i, m}\left(\pi^{\prime}\right)} \pi^{\prime}(s \mid X) & =\lambda^{0}(X) \frac{1}{n !} \sum_{s \in P^{i, m}\left(\pi^{\prime}\right)} \sum_{b \in B} \hat{\pi}\left(s^{b} \mid X\right) \stackrel{(2.24)}{=} \lambda^{0}(X) \frac{1}{n} \sum_{s \in P^{j, m}(\hat{\pi})} \hat{\pi}(s \mid X) \\
& \geq \lambda^{0}(Y) \frac{1}{n} \sum_{s \in P^{j, m}(\hat{\pi})} \hat{\pi}(s \mid Y)=\lambda^{0}(Y) \sum_{s \in P^{i, m}\left(\pi^{\prime}\right)} \pi^{\prime}(s \mid Y),
\end{aligned}
$$

where the inequality comes from Lemma 2.11 and the fact that $\alpha^{\hat{\pi}}$ is a BNE. A similar argument holds for $m^{\prime} \in M_{i}^{y}\left(\pi^{\prime}\right)$. It follows that $\alpha^{\pi^{\prime}}$ is a BNE. Thus, we can assume without loss of generality that $\hat{\pi}$ is anonymous.

Recall that for each action profile $a \in A, S^{a}(\hat{\pi})=\left\{s \in S^{\hat{\pi}} \mid \alpha^{\hat{\pi}}(s)=a\right\}$. For each $a \in A$ and $\omega \in \Omega$, define $\pi(a \mid \omega)=\sum_{s \in S^{a}(\hat{\pi})} \hat{\pi}(s \mid \omega)$. We showed in the proof of Lemma 2.23 that $\pi$ is straightforward and $V^{\pi}\left(\lambda^{0}\right)=V^{\hat{\pi}}\left(\lambda^{0}\right)$. So, what remains to show is that $\alpha^{\pi}$ is a BNE.

Claim: For all $i \in N$ it holds that

$$
\bigcup_{a \in P^{i, x}(\pi)} S^{a}(\hat{\pi})=\bigcup_{m \in M_{i}^{x}(\hat{\pi})} P^{i, m}(\hat{\pi}) .
$$


and the unions on both sides of the equation are over disjoint sets.

Proof. Let $s \in \bigcup_{a \in P^{i, x}(\pi)} S^{a}(\hat{\pi})$. Then, there exists $a \in S^{\pi}$ with $a_{i}=x$ such that $\alpha^{\hat{\pi}}(s)=a$ and $i$ is pivotal in $s$. Since $a_{i}=x$, we have $\alpha_{i}^{\hat{\pi}}\left(s_{i}\right)=a_{i}=x$, hence $s_{i} \in M_{i}^{x}(\hat{\pi})$, so $s \in \bigcup_{m \in M_{i}^{x}(\hat{\pi})} P^{i, m}(\hat{\pi})$. For the converse, let $s \in \bigcup_{m \in M_{i}^{x}(\hat{\pi})} P^{i, m}(\hat{\pi})$. Then, $s_{i} \in M_{i}^{x}(\hat{\pi})$ and $i$ is pivotal in $s$. Let $a=\alpha^{\hat{\pi}}(s)$, so that $s \in S^{a}(\hat{\pi})$. Since $s_{i} \in M_{i}^{x}(\hat{\pi})$, we have $a_{i}=\alpha_{i}^{\hat{\pi}}\left(s_{i}\right)=x$, so that $a \in P^{i, x}(\hat{\pi})$, hence $s \in \bigcup_{a \in P^{i, x}(\pi)} S^{a}(\hat{\pi})$. For the same reasons as in the proof of Lemma 2.5 both unions are over disjoint sets.

Since the unions on both sides of (2.25) are disjoint, we have

$$
\begin{aligned}
\lambda^{0}(X) \sum_{a \in P^{i, x}(\pi)} \pi(a \mid X) & =\lambda^{0}(X) \sum_{a \in P^{i, x}(\pi)} \sum_{t \in S^{a}(\hat{\pi})} \hat{\pi}(t \mid X) \stackrel{(2.25)}{=} \lambda^{0}(X) \sum_{m \in M_{i}^{x}(\hat{\pi})} \sum_{s \in P^{i, m}(\hat{\pi})} \hat{\pi}(s \mid X) \\
& \geq \lambda^{0}(Y) \sum_{m \in M_{i}^{x}(\hat{\pi})} \sum_{s \in P^{i, m}(\hat{\pi})} \hat{\pi}(s \mid Y)=\lambda^{0}(Y) \sum_{a \in P^{i, x}(\pi)} \pi(a \mid Y),
\end{aligned}
$$

where the inequality holds since $\alpha^{\hat{\pi}}$ is a BNE. Showing that choosing action $y$ is optimal upon observing message $y$ is similar. It follows that $\pi \in \Pi^{\text {sae }}$.

Proof of Lemma 2.15. If $\lambda^{0}(X) \geq 1 / 2$, then consider $\pi \in \Pi^{\text {sae }}$ with representation $(q, r)$ such that $q_{n}=1$ and $r_{k+1}=1$. Observe that $V^{\pi}\left(\lambda^{0}\right)=1 \geq V^{\hat{\pi}}\left(\lambda^{0}\right)$. Let $\lambda^{0}(X)<1 / 2$. Let $\hat{\pi}$ have representation $(\hat{q}, \hat{r})$ and let $\pi \in \Pi^{\mathrm{a}}$ be defined such that $q_{k-1}=q_{k}=0, q_{k+1}=$ $\hat{q}_{k-1}+\hat{q}_{k}+\hat{q}_{k+1}, q_{\ell}=\hat{q}_{\ell}$ for all $\ell \neq k-1, k, k+1, r_{k}=0, r_{k+1}=\hat{r}_{k}+\hat{r}_{k+1}$, and $r_{\ell}=\hat{r}_{\ell}$ for all $\ell \neq k, k+1$. Clearly, $\pi$ satisfies (2.16) and (2.17), so $\pi \in \Pi^{\text {ae }}$. We next show that $\pi$ is straightforward. Since $\hat{\pi}$ is straightforward, it holds that $\lambda^{0}(Y) \sum_{\ell=0}^{n} \frac{\ell}{n} \hat{r}_{\ell} \leq \lambda^{0}(X) \sum_{\ell=0}^{n} \frac{\ell}{n} \hat{q}_{\ell}$. Moreover, as $\alpha^{\hat{\pi}}$ is a BNE, it holds that $\lambda^{0}(Y) \hat{r}_{k} \leq \lambda^{0}(X) \hat{q}_{k}$. Hence,

$$
\begin{aligned}
\lambda^{0}(Y) \sum_{\ell=0}^{n} \frac{\ell}{n} r_{\ell} & =\lambda^{0}(Y)\left(\sum_{\ell=0}^{n} \frac{\ell}{n} \hat{r}_{\ell}+\frac{1}{n} \hat{r}_{k}\right) \leq \lambda^{0}(X)\left(\sum_{\ell=0}^{n} \frac{\ell}{n} \hat{q}_{\ell}+\frac{1}{n} \hat{q}_{k}+\frac{2}{n} \hat{q}_{k-1}\right) \\
& =\lambda^{0}(X) \sum_{\ell=0}^{n} \frac{\ell}{n} q_{\ell},
\end{aligned}
$$

which proves that $\pi \in \Pi^{\text {sae }}$. Finally,

$$
V^{\pi}\left(\lambda^{0}\right)=\lambda^{0}(X) \sum_{\ell=k}^{n} q_{\ell}+\lambda^{0}(Y) \sum_{\ell=k}^{n} r_{\ell} \geq \lambda^{0}(X) \sum_{\ell=k}^{n} \hat{q}_{\ell}+\lambda^{0}(Y) \sum_{\ell=k}^{n} \hat{r}_{\ell}=V^{\hat{\pi}}\left(\lambda^{0}\right),
$$

which completes the proof.

Proof of Lemma 2.16. If $\lambda^{0}(X) \geq 1 / 2$, then consider $\pi \in \Pi^{\text {sae }}$ with representation $(q, r)$ such that $q_{n}=1$ and $r_{k+1}=1$. Observe that $V^{\pi}\left(\lambda^{0}\right)=1 \geq V^{\hat{\pi}}\left(\lambda^{0}\right)$. Let $\lambda^{0}(X)<1 / 2$. By Lemma 2.15, we can assume without loss of generality that $\hat{q}_{k}=\hat{r}_{k}=0$, where $(\hat{q}, \hat{r})$ is the 
representation of $\hat{\pi}$. Let $\pi \in \Pi^{\mathrm{a}}$ be defined by $q_{n}=1, q_{\ell}=0$ for all $\ell \neq n, r_{0}=\sum_{\ell=0}^{k-1} \hat{r}_{\ell}$, $r_{k+1}=\sum_{\ell=k+1}^{n} \hat{r}_{\ell}$ and $r_{\ell}=0$ for all $\ell \neq 0, k+1$. Then $\pi \in \Pi^{\text {sa }}$ since

$$
\begin{aligned}
\lambda^{0}(Y) \pi_{i}(x \mid Y) & =\lambda^{0}(Y) \sum_{\ell=0}^{n} \frac{\ell}{n} r_{\ell}=\lambda^{0}(Y) \frac{k+1}{n} r_{k+1}=\lambda^{0}(Y) \frac{k+1}{n} \sum_{\ell=k+1}^{n} \hat{r}_{\ell} \leq \lambda^{0}(Y) \sum_{\ell=0}^{n} \frac{\ell}{n} \hat{r}_{\ell} \\
& \leq \lambda^{0}(X) \sum_{\ell=0}^{n} \frac{\ell}{n} \hat{q}_{\ell} \leq \lambda^{0}(X)=\lambda^{0}(X) \pi_{i}(x \mid X),
\end{aligned}
$$

where the second inequality holds as $\hat{\pi} \in \Pi^{\text {sae }}$. Since $q_{k-1}=q_{k}=r_{k}=0,(2.16)$ and (2.17) are satisfied. So, $\pi \in \Pi^{\text {sae }}$. Finally,

$$
V^{\pi}\left(\lambda^{0}\right)=\lambda^{0}(X)+\lambda^{0}(Y) r_{k+1} \geq \lambda^{0}(X) \sum_{\ell=k}^{n} \hat{q}_{\ell}+\lambda^{0}(Y) \sum_{\ell=k+1}^{n} \hat{r}_{\ell}=V^{\hat{\pi}}\left(\lambda^{0}\right),
$$

where the last equality uses that $\hat{r}_{k}=0$.

Proof of Proposition 2.17. If $\lambda^{0}(X) \geq 1 / 2$, then the solution to the linear optimization problem in Proposition 2.17 is given by $\left(r_{0}^{*}, r_{k+1}^{*}\right)=(0,1)$, leading to $V^{* \mathrm{e}}\left(\lambda^{0}\right)=1$ as desired. Let $\lambda^{0}(X)<1 / 2$. By Lemmas 2.14, 2.15, and 2.16, if there is an optimal $\hat{\pi} \in \Pi^{\text {sae }}$ then there is $\pi \in \Pi^{\text {sae }}$ with representation $(q, r)$ such that $q_{k}=1, r_{\ell}=0$ for all $\ell \neq 0, k+1$, and $V^{*}\left(\lambda^{0}\right)=\lambda^{0}(X)+\lambda^{0}(Y) r_{k+1}$. Such $\pi$ is straightforward if and only if

$$
\lambda^{0}(X)=\lambda^{0}(X) \pi_{i}(x \mid X) \geq \lambda^{0}(Y) \pi_{i}(x \mid Y)=\lambda^{0}(Y) \frac{k+1}{n} r_{k+1},
$$

which is equivalent to $(2.21)$.

Proof of Theorem 2.18. By (2.21), it holds that

$$
\frac{\lambda^{0}(X)}{\lambda^{0}(Y)} \frac{n}{k+1} \geq r_{k+1}
$$

Since the objective function is increasing in $r_{k+1}$, the optimal value is obtained by choosing

$$
r_{k+1}^{*}= \begin{cases}1 & \text { if } \lambda^{0}(X) \geq \frac{k+1}{n+k+1} \\ \frac{\lambda^{0}(X)}{\lambda^{0}(Y)} \frac{n}{k+1} & \text { if } \lambda^{0}(X)<\frac{k+1}{n+k+1}\end{cases}
$$

so if $\lambda^{0}(X) \geq \frac{k+1}{n+k+1}$, then $V^{* e}\left(\lambda^{0}\right)=1$. Otherwise, it holds that

$$
V^{* \mathrm{e}}\left(\lambda^{0}\right)=\lambda^{0}(X)+\lambda^{0}(Y) r_{k+1}=\lambda^{0}(X)+\lambda^{0}(Y) \frac{\lambda^{0}(X)}{\lambda^{0}(Y)} \frac{n}{k+1}=\lambda^{0}(X) \frac{n+k+1}{k+1} .
$$

as required.

Proof of Corollary 2.20. 
(i) These inequalities follow immediately from the simple observation that, for all $k=$ $1, \ldots, n-1$,

$$
2 \leq \frac{n+k+1}{k+1} \leq \frac{n+k}{k}
$$

(ii) This follows immediately from Proposition 2.3 and Theorems 2.8 and 2.18.

Proof of Corollary 2.21. This follows immediately from the observation that $(n+k+$ $1) /(k+1)$ is decreasing in $k$ and bounded from below by 2 for all $k \leq n-1$. 


\section{Chapter 3}

\section{Bayesian Persuasion in \\ Communication Networks}

"And a new philosophy emerged called quantum physics, which suggest that the individual's function is to inform and be informed. You really exist only when you're in a field sharing and exchanging information."

- Timothy Leary 
In this chapter, we study a multiple-receiver Bayesian persuasion model, where a sender communicates with identical receivers. The sender wants to implement a proposal and commits to a communication strategy which sends private correlated messages to the receivers, who are in a fixed and commonly known network. Besides their own, receivers also observe their neighbors' private messages and vote sincerely. We find that such networks of shared information often do not restrict the sender's gain from persuasion with private communication compared to the empty network case. Surprisingly, the sender's value does not decrease monotonically as the network becomes denser.

\subsection{Introduction}

Multiple-receiver Bayesian persuasion models with private communication often assume that receivers do not exchange information with each other between receiving signals from the sender and taking their action. In reality, however, people usually deliberate before voting in favor of a political party or simply before buying a product, and might consult friends and acquaintances in search of additional opinions and information. We model such communication among receivers prior to making a decision with a simple setup: we assume that receivers are in a fixed network that is commonly known and that neighbors can observe each other's private messages. An application of such communication are social networks like Facebook, where parties can target political adverts at specific (potential) voter groups, and such use of social media has become a common feature of election campaigns in recent years. In this setting, if a person likes or shares an ad or a video, it is visible to all of their friends on the social network. In this paper we consider the limit case, where the sender sends perfectly custom-tailored messages to the receivers and the messages are observed by the receivers and their direct neighbors with probability one.

This setup significantly complicates the sender's problem of optimal persuasion as he must also take into account the intricacies of the information flow between receivers when deciding how to design his communication strategy. In the absence of a network structure, the sender can improve upon public communication by employing private messages. Hence, the immediate question arises whether private communication is still (strictly) beneficial for the sender when receivers communicate within a network. If so, are some networks more manipulable than others? Is the most manipulable network empty? Can the sender benefit from a denser network?

\subsubsection{An Illustrative Example}

Suppose that a company, which is either profitable $(\mathrm{P})$ or not profitable $(\mathrm{N})$, has two potential investors, $I_{1}$ and $I_{2}$, who initially believe it to be profitable with probability $1 / 3$. Investors 
will invest in the company only if they consider it is profitable with probability at least $1 / 2$. Both parties can potentially give the same amount, and the investment from only one of them is sufficient to cover the company's cash flow problems. Two financial reports are prepared regarding the company's finances, where each report is randomly assigned (with equal probability) to one of the investors. While one report provides true findings, the other one always favors investment.

First, assume that investors are not communicating, i.e. they are in the empty network. The communication strategy of the CEO can be formalized by distributions $\pi(\cdot \mid P)$ and $\pi(\cdot \mid N)$ on some set of signals. Let $(p, n)$ denote the signal in which $I_{1}$ receives a message to invest and $I_{2}$ a message not to invest. The CEO's communication strategy above can be represented as follows.

\begin{tabular}{c|cc}
$\pi$ & $P$ & $N$ \\
\hline$(p, p)$ & 1 & 0 \\
$(p, n)$ & 0 & $\frac{1}{2}$ \\
$(n, p)$ & 0 & $\frac{1}{2}$
\end{tabular}

After observing $p$, any investor's belief that the company is profitable is $1 / 2$. Hence, after any realization at least one of them invests. The CEO guarantees at least one investment with probability 1 and the communication strategy above is indeed optimal.

Now, assume that $I_{1}$ and $I_{2}$ know each other and exchange the information they got from the reports before making their decisions. This can be presented with the network:

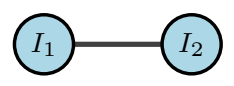

Since investors can observe each other's messages, the communication strategy above is no longer optimal: when the signal realization is $(p, n)$ or $(n, p)$, both investors know that the company is not profitable, i.e. the true state is $N$. In this case, the probability of obtaining an investment is determined by the probability of $(p, p)$, i.e. $1 / 3$. Since receivers can observe the signal realization (as the network is complete), the CEO cannot communicate privately and alternatively chooses the following communication strategy:

\begin{tabular}{c|cc}
$\pi^{\prime}$ & $P$ & $N$ \\
\hline$(p, p)$ & 1 & $\frac{1}{2}$ \\
$(n, n)$ & 0 & $\frac{1}{2}$
\end{tabular}

Note that since the communication strategy is public, either both investors invest or none of them does. The probability of obtaining an investment under $\pi^{\prime}$ is $1 / 3 \cdot 1+2 / 3 \cdot 1 / 2=2 / 3$. Since it is sufficient for the CEO to guarantee only one investment, the CEO is worse off when the investors are communicating. 
The example illustrates how the additional exchange of information affects the Sender's gain from persuasion in two extreme cases: the empty network when there is no link between $I_{1}$ and $I_{2}$, and the complete network in the latter case. In a complete network, it is clear that the sender's optimal communication strategy is public. This paper shows that in some networks that are neither empty nor complete (and for some voting quotas) the sender's optimal communication strategy is indeed public. Later we show that optimal communication can be public in some networks that are not complete, but in general the sender benefits from private communication.

\subsubsection{Overview of Results}

We consider an exogenous network that is common knowledge, a binary state space, and a sender who commits to a (private) communication strategy. The sender wishes to implement a certain outcome and does not care about the true state. Receivers know the joint distribution of signals (vectors of messages), but only observe their own and their neighbors' private messages from the signal realization. Taking all observations into account, receivers update their beliefs via Bayes rule. If the network is empty, our model reduces to the model in CHAPTER 2, which is used as a benchmark.

First, we show that for non-empty networks the "traditional" assumptions for multiplereceiver Bayesian persuasion models are no longer without loss of generality. The most common of these assumptions, straightforwardness, under which the sender sends "recommendations" to vote for an alternative and receivers follow the recommendations, does not hold since receivers take into account not only their own messages, but also their neighbors'. The shared information limits the possible message combinations the sender can use for optimal persuasion as the recommendations are no longer fully private.

Another common assumption that is not without loss of generality is "truth-telling" in the sender's preferred state. In other words, it is not always in the sender's best interest to generate a perfectly informative message whenever the true state is his preferred one, i.e. there exist networks (e.g. the line network) where the sender is better off garbling information in such cases. This is due to the fact that, in addition to different messages, the variability between the signals in the sender's preferred state is used to limit the information flow between neighbours.

Given any number of receivers and any quota, the sender cannot achieve a higher probability of success in a non-empty network than in the empty network of the same size. The reason for this is that given any non-empty network and any communication strategy $\pi$, there exists a communication strategy $\pi^{\prime}$ which reveals exactly the same information in the empty network as $\pi$ does in the non-empty network. In other words, any communication strategy in a non-empty network can be replicated in its corresponding empty network. In contrast, 
when the network is complete, the optimal communication strategy is public, as illustrated in the motivating example. For networks that lie in between the empty network and the complete network, we consider network structures which are often discussed in the literature (star, wheel, circle and other regular networks) and identify conditions under which the sender can achieve the probability of success as under the empty network. Interestingly, if there are sufficiently many isolated nodes in a network, this can be achieved regardless of how many or between which of the remaining receivers the links are.

Finally, perhaps surprisingly, while it seems intuitive that adding a link to a given network would (weakly) decrease the sender's gain from persuasion as receivers gain more information, in our model it is possible that the sender is better off in a denser network. The reason is that while any communication strategy in a non-empty network can be replicated in the empty network of the same size, it is not always the case that it can be replicated in a less dense network which is non-empty.

The rest of the paper is organized as follows. Subsection 3.1.3 discusses related literature. Section 3.2 provides notation and preliminary definitions. Section 3.3 discusses the benchmark case and preliminary results. Section 3.4 gives observations which hold for network structures in general. Section 3.5 focuses on optimality in specific networks. Section 3.6 shows that a denser network is not always worse for the sender and Section 3.7 concludes. All proofs can be found in Appendix 3.8.

\subsubsection{Related Literature}

The current model comes closest to and is an extension of Kerman et al. (2020), which builds upon Kamenica and Gentzkow (2011) and considers a sender communicating privately with multiple receivers who wishes to persuade a certain number of them with the greatest probability of success. The authors derive an optimal communication strategy under the assumption that receivers vote sincerely. While we also assume sincere voting, a crucial difference to the current setup is that in their model a receiver only has access to information revealed by the sender, whereas in our setup directly connected voters perfectly exchange information. Thus, their model is a special case of ours (the empty network). While they focus on finding an optimal communication strategy that yields an equilibrium under sincere voting, we determine optimal communication strategies for different networks.

Bayesian persuasion in networks is a relatively new area of research and up until now there have been several studies that involve a network structure. The closest study to ours in this regard is Galperti and Perego (2020), which considers a model of information spillovers in a network, whereby a designer targets certain agents with private signals about the state of the world, after which the information spreads from the targeted agents in the network. A difference with our model lies in the sender being able to target certain agents in the network, 
whereas we assume that the sender communicates directly with all agents in the network. Furthermore, they assume that information diffuses from the targeted agents to all agents in the network to which a path exists. In contrast, in our model information is only shared with direct neighbors, which calls for a different approach.

Another paper that studies Bayesian persuasion in networks is Egorov and Sonin (2019), where a sender communicates publicly with the receivers who are in a fixed network by choosing the level of propaganda. A receiver either gets the information from the sender for a cost, or relies on his neighbors for the information. In contrast, we consider private communication and costless access to information. Candogan and Drakopoulos (2020) consider a model of social network interactions in which agents choose whether or not to engage with some (possibly inaccurate) content on the social network and the agents' payoffs depend on the engagement of their neighbors. The platform designs a signalling mechanism which maximizes engagement or minimizes misinformation by sending recommendations to its users to engage or not. In another model with local strategic complementarities Candogan (2019) finds that when the degrees of some nodes in the network increase, this can reduce the information designer's payoff.

Our paper also shares some characteristics with Buechel and Mechtenberg (2019), where agents are in a network and experts can share with their direct neighbors a policy recommendation on the basis of private signals they receive. Unlike our setup, they have strategic receivers and agents decide whether or not to share information with their neighborhood, i.e. they can ignore connections. Another related paper that considers a sender-receiver model with information transmission in networks is Jiménez-Martínez (2015). While he studies how receivers' beliefs are influenced by each other, an important difference is that the network is weighted and directed; he shows that reaching consensus in the society depends on "prominent" agents in the network.

The current paper relates to the literature on public communication and collective decision making. Schnakenberg (2015) considers an expert who is privately informed about the state and can conceal information from the receivers. ${ }^{1}$ One important distinction from our paper is the absence of the commitment assumption, which in effect reduces the ex ante expected utilities of the voters. Alonso and Câmara (2016) study public communication where receivers have heterogeneous preferences which the sender can exploit by targeting minimal winning coalitions. Kosterina (2018) considers a model with a continuous state space in which the receiver's prior is unknown. She shows that even under this ambiguity, the optimal communication strategy always includes a signal that tells the truth with probability 1 , whereas in our setup this is not generally true.

\footnotetext{
${ }^{1}$ Another similar extension to the standard model involves a privately informed receiver (Kolotilin et al., 2017; Hu and Weng, 2020; Bizzotto and Vigier, 2020).
} 
Our paper also relates to the literature on voting games and private communication. Wang (2013) compares the outcomes from public and private communication under strategic voting and concludes that the sender is weakly worse off under private communication when messages are uncorrelated. In contrast, we consider private correlated messages and show that this often improves upon public communication. Bardhi and Guo (2018) also focus on collective decision making and, in particular, the unanimity voting rule, whereas we allow for general monotonic and anonymous voting rules. Chan, Gupta, Li, and Wang (2019) consider private communication when voting is costly. In their model the optimal communication strategy targets the receivers with the lowest costs who are easiest to persuade. While Arieli and Babichenko (2019) do not consider collective decision making, they focus on private communication and an advertiser who wishes to maximize the number of adoptions of a product. In particular, they characterize optimal communication for different utility functions of the sender.

This paper is also related to the literature on more general games in information design. Bergemann and Morris (2016a) consider a game of incomplete information, characterize Bayesian Nash equilibria, and demonstrate that this corresponds to Bayes correlated equilibria. They show that in equilibrium receivers are "obedient"; they follow the recommendation by the sender. Taneva (2019) derives the optimal information structure in finite environments and characterizes it in a symmetric binary setting. She shows that a related notion dubbed "directness" (which corresponds to straightforwardness à la Kamenica and Gentzkow (2011)) can be used without loss of generality. One important difference of our paper to these is that our setting does not generally allow for straightforwardness.

\subsection{Notation}

\subsubsection{Communication Strategy}

Let $N=\{1, \ldots, n\}$ be the set of receivers and $\Omega=\{X, Y\}$ the set of states of the world. For any set $X$ denote by $\Delta(X)$ the set of probability distributions over $X$ with finite support. Sender and receivers share a common prior belief $\lambda^{0} \in \Delta^{\circ}(\Omega)$ about the true state of the world, where $\Delta^{\circ}(\Omega)$ denotes the set of strictly positive probability distributions on $\Omega$.

Let $S_{i}$ be a finite set of messages the sender can send to receiver $i$, and let $S=\prod_{i \in N} S_{i}$, where the elements of $S$ are called signals. A communication strategy is a function $\pi$ : $\Omega \rightarrow \Delta(S)$ which maps each state of the world to a joint probability distribution over signal realizations. Denote the set of all communication strategies by $\Pi$.

For each signal $s \in S$, let $s_{i} \in S_{i}$ denote the message for receiver $i$. For each $s_{i} \in S_{i}$ and $\omega \in \Omega$, let $\pi_{i}\left(s_{i} \mid \omega\right)=\sum_{t \in S: t_{i}=s_{i}} \pi(t \mid \omega)$, which is the probability that receiver $i$ observes $s_{i}$ given $\omega$. Define $S^{\pi}=\{s \in S \mid \exists \omega \in \Omega: \pi(s \mid \omega)>0\}$. That is, $S^{\pi}$ consists of signals in $S$ which are sent with positive probability by $\pi$. Similarly, for each $\pi$ and $i \in N$, define 
$S_{i}^{\pi}=\left\{s_{i} \in S_{i} \mid \exists \omega \in \Omega: \pi_{i}\left(s_{i} \mid \omega\right)>0\right\}$, which is the set of messages receiver $i$ observes with positive probability under $\pi$.

\subsubsection{Networks}

An undirected network is a map $g: N \times N \rightarrow\{0,1\}$ with $g_{i j}=g(i, j)$ and $g_{i j}=g_{j i}$. Given a set of receivers $N$, let $G(N)$ be the set of all such networks. We assume that receivers are in a fixed network, which is common knowledge among the sender and receivers. Each receiver in the network can observe his neighbors' message realizations. Thus, in a non-empty network a receiver gathers more information about the true state than he would from the same communication strategy under the empty network.

A network $g \in G(N)$ is complete if for all $i, j \in N$ with $i \neq j$ it holds that $g_{i j}=1$, i.e. every two nodes have a link. In this case each receiver knows the signal realization, so all communication strategies are public on the complete network. For any network $g \in G(N)$, we denote the empty network with the same number of receivers by $g_{0}$. Observe that an empty network corresponds to a standard multiple-receiver Bayesian persuasion model.

Let $N_{i}(g)=\left\{j \in N \mid g_{i j}=1\right\}$ be the neighborhood of receiver $i$ in $g$ and let $\delta_{i}^{g}=\left|N_{i}(g)\right|$ denote the degree of $i$ in $g$. Let $\bar{N}_{i}(g)=N_{i}(g) \cup\{i\}$. For any $\pi \in \Pi, s \in S^{\pi}, i \in N$, and $j \in N_{i}(g)$, let $s_{i j}$ be the message $i$ observes from $j$ in $s$, that is $s_{i j}=s_{j}$. Let $s_{i}(g)=$ $\left(s_{i j}\right)_{j \in \bar{N}_{i}(g)}$ be the information neigborhood of receiver $i$ in $s$, that is, $s_{i}(g)$ is the vector of messages (with length $\delta_{i}^{g}+1$ ) receiver $i$ observes upon signal realization $s$. Let $A_{i}^{\pi}(g, s)=$ $\left\{t \in S^{\pi} \mid t_{i}(g)=s_{i}(g)\right\}$ be the set of signals $i$ considers possible upon signal realization $s$, or in other words, the set of signals $i$ associates with $s$. Given $s, t \in S^{\pi}$, we say that $t$ is associated with $s$ if there exists an agent $i \in N$ such that $t \in A_{i}^{\pi}(g, s)$. Let $A^{\pi}(g, s)=\cup_{i \in N} A_{i}^{\pi}(g, s)$ be the set of all signals associated with $s$.

For any $g \in G(N), \pi \in \Pi$, and $s \in S^{\pi}$, the posterior belief vector $\lambda^{s, g} \in \Delta(\Omega)^{n}$ is defined by:

$$
\lambda_{i}^{s, g}(\omega)=\frac{\sum_{t \in A_{i}^{\pi}(g, s)} \pi(t \mid \omega) \lambda^{0}(\omega)}{\sum_{\omega^{\prime} \in \Omega} \sum_{t \in A_{i}^{\pi}(g, s)} \pi\left(t \mid \omega^{\prime}\right) \lambda^{0}\left(\omega^{\prime}\right)}, \quad i \in N, \omega \in \Omega
$$

That is, $\lambda_{i}^{s, g}(\omega)$ is receiver $i$ 's posterior belief that the state is $\omega$ upon observing $s_{i}(g)$.

A communication strategy $\pi \in \Pi$ induces $\sigma^{g} \in \Delta\left(\Delta(\Omega)^{n}\right)$ under network $g$ if for all $\lambda \in \Delta(\Omega)^{n}$ it holds that

$$
\sigma^{g}(\lambda)=\sum_{s \in S^{\pi}: \lambda^{s, g}=\lambda} \sum_{\omega \in \Omega} \pi(s \mid \omega) \lambda^{0}(\omega)
$$

In words, $\sigma^{g}(\lambda)$ is the probability of posterior vector $\lambda$ under network $g .^{2}$

\footnotetext{
${ }^{2}$ If $\lambda \notin \operatorname{supp}\left(\sigma^{g}\right)$, then the right hand side of (3.2) is 0 . Moreover, it is well-defined since $S^{\pi}$ is finite.
} 


\subsubsection{Voting}

For each $i \in N$, let $B_{i}=\{x, y\}$ be the set of actions of receiver $i$. Let $B=\prod_{i \in N} B_{i}$ denote the space of action profiles and let $Z=\{x, y\}$ be the set of voting outcomes. Following the signal realization, each receiver chooses an action according to his posterior belief.

Let $z^{k}: B \rightarrow Z$ be a map, where $z^{k}(a)$ is the outcome of the vote when the action profile is $a$ and is defined by:

$$
z^{k}(a)= \begin{cases}x & \text { if }\left|\left\{i \in N: a_{i}=x\right\}\right| \geq k \\ y & \text { otherwise }\end{cases}
$$

We assume that the sender's utility function $v: Z \rightarrow\{0,1\}$ has value 1 if $x$ is implemented and 0 otherwise. For each $i \in N$, let $u_{i}: Z \times \Omega \rightarrow\{0,1\}$ be the utility function of receiver $i$ such that $u_{i}(x, X)=u_{i}(y, Y)=1$ and $u_{i}(x, Y)=u_{i}(y, X)=0$.

To keep the model simple and to focus more on the effects of a network on persuasion, we assume that the receivers vote sincerely. $^{3}$ For any $g \in G(N), \pi \in \Pi$, and $i \in N$, let $S_{i}^{\pi}(g)=\prod_{j \in \bar{N}_{i}(g)} S_{j}^{\pi}$ be the space of vectors of length $\delta_{i}^{g}+1$ that $i$ can observe under $g$ and $\pi$. Let $\alpha_{i}^{\pi, g}: S_{i}^{\pi}(g) \rightarrow B_{i}$ be agent $i$ 's sincere action function, such that for any realization $s \in S^{\pi}$ it holds that:

$$
\alpha_{i}^{\pi, g}\left(s_{i}(g)\right)= \begin{cases}x & \text { if } \lambda_{i}^{s, g}(X) \geq \frac{1}{2}, \\ y & \text { otherwise. }\end{cases}
$$

That is, a receiver chooses action $x$ if she believes the true state is $X$ with at least probability $1 / 2$. Throughout the paper we assume that $\lambda^{0}(X)<\lambda^{0}(Y)$, since otherwise there is no need for persuasion. Define $Z_{x}^{g}(\pi)=\left\{s \in S^{\pi} \mid z^{k}\left(\alpha^{\pi, g}(s)\right)=x\right\}$. That is, $Z_{x}^{g}(\pi)$ is the set of signals which implement $x$ in $g$ under $\pi$ and sincere voting.

Receiver $i$ is pivotal in $s \in S^{\pi}$ if for any $a_{i} \in B_{i}, z^{k}\left(a_{i}, \alpha_{-i}^{\pi, g}\left(s_{-i}(g)\right)=a_{i}\right.$. That is, $i$ is pivotal in the voting following $s$ if $i$ 's vote determines the voting outcome given that all $j \neq i$ vote sincerely.

Let $a \in B$ be an action profile and $z=z^{k}(a)$ be a voting outcome. The value of a communication strategy $\pi \in \Pi$ for quota $k$ is defined as the sender's expected utility under distribution $\sigma^{g}$ induced by $\pi$ in network $g$. As we fix $\lambda^{0}$ and $\alpha_{i}^{\pi, g}$ throughout the paper, we write $V_{k}^{\pi}(g)=V_{k}^{\pi}\left(\lambda^{0}, g, \alpha^{\pi, g}\right)$. The value of the sender is:

$$
V_{k}^{\pi}(g)=\mathbb{E}_{\lambda^{0}}\left[\mathbb{E}_{\pi}\left[v\left(z^{k}\left(\alpha^{\pi, g}(s)\right)\right]\right]=\lambda^{0}(X) \sum_{s \in Z_{x}^{g}(\pi)} \pi(s \mid X)+\lambda^{0}(Y) \sum_{s \in Z_{x}^{g}(\pi)} \pi(s \mid Y) .\right.
$$

Thus, the value of a communication strategy is equal to the probability of $x$ being implemented under $\pi$ and $g$ and quota $k$, given that receivers vote sincerely.

\footnotetext{
${ }^{3}$ While this assumption makes the model much more tractable, it can also be supported by empirical papers which show that voters behave sincerely under certain conditions (Felsenthal and Brichta, 1985; Degan and Merlo, 2007; Groseclose and Milyo, 2010).
} 
A communication strategy $\pi^{*}$ is optimal in $\Pi$ under $g$ for quota $k$ if $V_{k}^{\pi^{*}}(g)=\sup _{\pi \in \Pi} V_{k}^{\pi}(g)$. The value of an optimal communication strategy on the empty network with $n$ nodes and quota $k$ is denoted by $V_{k}^{n}$.

\subsection{Preliminaries}

This section demonstrates how the information-sharing feature of our model produces a nontrivial change in the overall setup of multiple-receiver Bayesian persuasion as many of the common assumptions in standard models cease to hold in general. First, we briefly consider the model in Kerman et al. (2020), which corresponds to the empty network case in our framework and is used as a benchmark. Second, we demonstrate that the network structure can affect the agents' posteriors and the (optimal) value. Third, we show that the notion of straightforwardness as defined in Kamenica and Gentzkow (2011) does not hold for optimal strategies in our model. Finally, sending a recommendation with probability 1 in the sender's preferred state, an assumption that is made without loss of generality for optimal strategies in many Bayesian persuasion models, is also no longer optimal.

\subsubsection{Optimal Public Communication on the Empty Network}

In CHAPter 2, it is without loss of generality to restrict attention to straightforward (in the sense of Kamenica and Gentzkow (2011)) and anonymous communication strategies. Given a binary state space $\Omega=\{X, Y\}$, it is optimal for the sender to send "recommendations", $x$ or $y$, as messages. In this case, the probability that $x$ is sent to exactly $\ell$ receivers if the state is $X$ and the probability that $x$ is sent to exactly $\ell$ receivers if the state is $Y$ can be represented by $q_{\ell}$ and $r_{\ell}$, respectively, where each signal in which the same number of receivers observe $x$ has the same probability. An optimal communication strategy (in the empty network) is then given by Theorem 2.8 in CHAPTER 2. That is, adapting to this chapter's notation, we have $V_{k}^{n}=\min \left\{\frac{n+k}{k} \lambda^{0}(X), 1\right\}$.

Note that the optimal public communication strategy's value is always independent of the network and the quota since all agents observe the signal realization. In this case, since receivers have homogenous prior beliefs, the situation is the same as persuading a single receiver. Thus, as in Kamenica and Gentzkow (2011), $x$ is implemented with probability 1 in state $X$ and with probability $\lambda^{0}(X) / \lambda^{0}(Y)$ in state $Y$. The value of the optimal public communication strategy is then given by $V^{p}=\lambda^{0}(X) \cdot 1+\lambda^{0}(Y) \cdot \lambda^{0}(X) / \lambda^{0}(Y)=2 \lambda^{0}(X)$. 


\subsubsection{The Effects of the Network}

One important observation in our framework is that the set of posteriors which can be induced differs from the one under a model without a network structure. Given a communication strategy $\pi \in \Pi, s \in S^{\pi}$ and $g, g^{\prime} \in G(N)$, let $\lambda^{s, g}$ and $\lambda^{s, g^{\prime}}$ be the posterior vectors under $g$ and $g^{\prime}$, respectively. It is easy to see that for $g \neq g^{\prime}$, it is possible that $\lambda^{s, g} \neq \lambda^{s, g^{\prime}}$. If $g \subseteq g^{\prime}$, an agent gathers more information upon a signal realization under $g^{\prime}$ than under $g$ by also observing the messages of his new neighbors, which leads to different posterior beliefs in the two models. This is shown in Example 3.1.

Example 3.1. Let $|N|=3, \lambda^{0}(X)=1 / 3$, and $k=2$. The optimal communication strategy $\pi$ prescribed by Theorem 2.8 for the empty network $g_{0}$ is given by $q_{3}^{*}=1, r_{2}^{*}=3 / 4$, and $r_{0}^{*}=1 / 4$, since $\lambda^{0}(X)<k /(n+k)$. This can be represented by:

\begin{tabular}{cc|cc} 
& $\pi$ & $\omega=X$ & $\omega=Y$ \\
\cline { 2 - 3 }$(3, x, x)$ & 1 & 0 \\
$(3, x, y)$ & 0 & $\frac{1}{4}$ \\
& $(x, y, x)$ & 0 & $\frac{1}{4}$ \\
Network $g_{0}$ & $(y, x, x)$ & 0 & $\frac{1}{4}$ \\
& $(y, y, y)$ & 0 & $\frac{1}{4}$
\end{tabular}

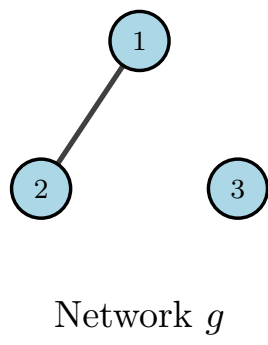

The support of the distribution that $\pi$ induces under the empty network $g_{0}$ is given by:

$$
\operatorname{supp}\left(\sigma^{g_{0}}\right)=\left\{\left(\frac{1}{2}, \frac{1}{2}, \frac{1}{2}\right),\left(\frac{1}{2}, \frac{1}{2}, 0\right),\left(\frac{1}{2}, 0, \frac{1}{2}\right),\left(0, \frac{1}{2}, \frac{1}{2}\right),(0,0,0)\right\},
$$

and the value is $V_{2}^{3}=V_{2}^{\pi}\left(g_{0}\right)=5 / 6$.

Now consider network $g$ on the right-hand side, which has one added link compared to its counterpart $g_{0}$, so that receivers 1 and 2 can observe each other's messages. The set of posteriors induced by $\pi$ under $g$ is:

$$
\operatorname{supp}\left(\sigma^{g}\right)=\left\{\left(\frac{2}{3}, \frac{2}{3}, \frac{1}{2}\right),\left(\frac{2}{3}, \frac{2}{3}, 0\right),\left(0,0, \frac{1}{2}\right),(0,0,0)\right\} .
$$

The value is $V_{2}^{\pi}(g)=1 / 2<5 / 6=V_{2}^{\pi}\left(g_{0}\right)$. There are fewer posterior vectors under $g$ than under the empty network $g_{0}$. Since receivers 1 and 2 can observe each other's messages, different signals lead to the same posterior vector. Note that 1 and 2 have the same posterior belief in any communication strategy. Since they have the same information neighborhood, they always update their beliefs in the same way. It follows that the sender can send the same message to 1 and 2 without loss of generality. ${ }^{4}$ Thus, since $k=2$ the problem is equivalent to persuading either receivers 1 and 2 together, or persuading all receivers. However,

\footnotetext{
${ }^{4} \mathrm{~A}$ generalized version of this statement is proved later on in Proposition 3.11.
} 
this is tantamount to employing a public communication strategy in which case the network does not have an effect since all agents observe the same message. Therefore, the optimal communication strategy in $g$ is public and given by:

\begin{tabular}{c|cc}
$\pi^{\prime}$ & $\omega=X$ & $\omega=Y$ \\
\hline$(x, x, x)$ & 1 & $\frac{1}{2}$ \\
$(y, y, y)$ & 0 & $\frac{1}{2}$
\end{tabular}

Thus, the value is $V^{\pi^{\prime}}(g)=1 / 3 \cdot 1+2 / 3 \cdot 1 / 2=2 / 3=V^{p}$. So, if $|N|=3$ and $k=2$, having even one link between agents reduces the optimal value from the one under the empty network, $V_{2}^{3}$, to the value of the optimal public communication strategy, $V^{p}$.

An intuitive observation in Example 3.1 is that adding a link to the network while keeping $\pi$ (which is optimal on $g_{0}$ ) fixed decreases the value of $\pi$. However, it is also possible that the sender benefits from a denser network under a fixed $\pi$. We show this next.

Example 3.2. Let $|N|=5, \lambda^{0}(X)=1 / 3$ and $k=3$. Consider the following network $g$ and communication strategy $\pi$ :

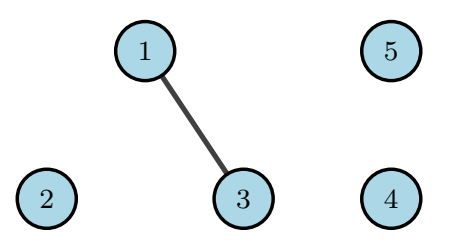

\begin{tabular}{c|cc}
$\pi$ & $\omega=X$ & $\omega=Y$ \\
\hline$(x, y, x, x, y)$ & $\frac{1}{3}$ & $\frac{1}{6}$ \\
$(x, y, x, y, x)$ & $\frac{1}{3}$ & $\frac{1}{6}$ \\
$(y, y, y, x, x)$ & $\frac{1}{6}$ & $\frac{1}{6}$ \\
$(y, y, y, y, y)$ & $\frac{1}{6}$ & $\frac{1}{2}$
\end{tabular}

The support of $\sigma^{g}$ is given by:

$$
\operatorname{supp}\left(\sigma^{g}\right)=\left\{\left(\frac{1}{2}, \frac{1}{3}, \frac{1}{2}, \frac{3}{7}, \frac{3}{11}\right),\left(\frac{1}{2}, \frac{1}{3}, \frac{1}{2}, \frac{3}{11}, \frac{3}{7}\right),\left(\frac{1}{5}, \frac{1}{3}, \frac{1}{5}, \frac{3}{7}, \frac{3}{7}\right),\left(\frac{1}{5}, \frac{1}{3}, \frac{1}{5}, \frac{3}{11}, \frac{3}{11}\right)\right\} .
$$

Thus, $x$ is not implemented after any realization, i.e. $V_{3}^{\pi}(g)=0$.

Now, consider the following network $g^{\prime}$.

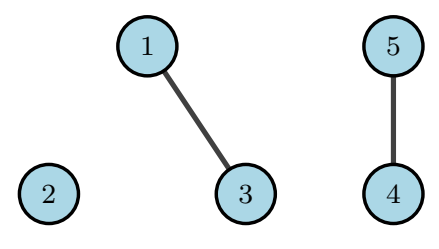

The beliefs of receivers 1, 2 and 3 are the same under $g^{\prime}$ as under $g$ but receivers 4 and 5 have different beliefs. The support under the same communication strategy $\pi$ is:

$$
\operatorname{supp}\left(\sigma^{g^{\prime}}\right)=\left\{\left(\frac{1}{2}, \frac{1}{3}, \frac{1}{2}, \frac{1}{2}, \frac{1}{2}\right),\left(\frac{1}{5}, \frac{1}{3}, \frac{1}{5}, \frac{1}{3}, \frac{1}{3}\right),\left(\frac{1}{5}, \frac{1}{3}, \frac{1}{5}, \frac{1}{7}, \frac{1}{7}\right)\right\} .
$$


Thus, $x$ is implemented after realizations $(x, y, x, x, y)$ and $(x, y, x, y, x)$ as both lead to the posterior vector $\left(\frac{1}{2}, \frac{1}{3}, \frac{1}{2}, \frac{1}{2}, \frac{1}{2}\right)$. Hence:

$$
V_{3}^{\pi}\left(g^{\prime}\right)=\frac{1}{3} \cdot \frac{2}{3}+\frac{2}{3} \cdot \frac{1}{3}=\frac{4}{9}>0=V_{3}^{\pi}(g),
$$

so, the sender benefits from the same communication strategy in the denser network $g^{\prime}$.

While the sender benefits from the additional connection provided in $g^{\prime}$, a notable aspect of Example 3.2 is that $\pi$ is not optimal on $g$. This leaves the question how adding a link generally affects the value of the optimal strategy. In Example 3.1, the optimal communication strategy $\pi$ under $g_{0}$ has a strictly higher value than the optimal communication strategy $\pi^{\prime}$ under the denser network $g$. One might expect this to be a general observation. However, Section 3.6 shows that this is not always true.

\subsubsection{Straightforwardness}

Translating the straightforwardness definition of Kamenica and Gentzkow (2011) to multiple receivers, a communication strategy $\pi \in \Pi$ is straightforward if $S_{i}^{\pi} \subseteq B_{i}$ and if for the sincere action function $\alpha_{i}^{\pi}: S_{i}^{\pi} \rightarrow B_{i}$ and $a_{i} \in S_{i}^{\pi}$ it holds that $\alpha_{i}^{\pi}\left(a_{i}\right)=a_{i}$. First, observe that under the sincere action function $\alpha_{i}^{\pi}$ receivers vote only according to their own message, whereas in our model the sincere action function $\alpha_{i}^{\pi, g}$ also takes into account their neighbors' messages. Hence, using the standard definition of straightforwardness is not without loss of generality. This observation is illustrated in Example 3.5 below. However, first we need to introduce a definition and a lemma.

Upon a signal realization, a receiver votes for $x$ if the probability of observing their specific message vector is sufficiently higher in state $X$ than in state $Y$, given their prior belief and a specified communication strategy. In order for this to hold, there must exist at least one signal which includes this message vector and has higher probability in state $X$. Such signals are instrumental for increasing the probability of implementing $x$ and will be referred to as "anchors", because the $x$ votes are dependent on them.

Definition 3.3. For any $\pi \in \Pi$ and $s \in S^{\pi}$, the signal $s$ is an anchor if $\pi(s \mid X) \lambda^{0}(X) \geq$ $\pi(s \mid Y) \lambda^{0}(Y)$. The set of all anchors is denoted by $A n(\pi)$.

Given $\pi \in \Pi, s \in A n(\pi)$ and $i \in N$, if for every $t \in S^{\pi}$ with $t \neq s$ it holds that $s_{i}(g) \neq t_{i}(g)$, then $\alpha_{i}^{\pi, g}\left(s_{i}(g)\right)=x$. That is, if a receiver $i$ can uniquely identify the signal realisation as an anchor, he votes for $x$.

Lemma 3.4. Let $g \in G(N)$. For any $\pi \in \Pi$ with $V_{k}^{\pi}(g)>0$ it holds that $A n(\pi) \neq \emptyset$. 
Lemma 3.4 shows that every communication strategy with a non-zero value has at least one anchor. Its proof further implies that every $x$-vote in such a strategy is associated with at least one anchor.

Now we can show that assuming straightforwardness is not without loss of generality. ${ }^{5}$

Example 3.5. Let $|N|=3, \lambda^{0}(X)=1 / 3$, and $k=1$. Consider the following network $g$ :

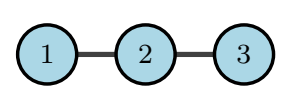

\begin{tabular}{c|cc}
$\pi^{\prime}$ & $\omega=X$ & $\omega=Y$ \\
\hline$(x, x, x)$ & 1 & 0 \\
$(y, x, x)$ & 0 & $\frac{1}{2}$ \\
$(x, x, y)$ & 0 & $\frac{1}{2}$
\end{tabular}

Let us try to construct a straightforward communication strategy $\pi$. First, note that $\lambda_{2}^{s, g}(X) \geq$ $1 / 2$ if and only if $s \in A n(\pi)$, so receiver 2 never votes for $x$ in any non-anchor signal. This is the case since for any $s \in S^{\pi}, s_{2}(g)=s$, that is, receiver 2 always observes the whole signal as he has access to the messages of everyone in the network. Therefore, for any straightforward communication strategy $\pi \in \Pi$ it must hold that: (i) $t_{2}=y$ for all $t \notin A n(\pi)$ and (ii) $s_{2}=x$ for all $s \in A n(\pi)$. However, since for any such $s$ and $t$ it holds that $s_{2} \neq t_{2}$, we have $\lambda_{1}^{t, g}(X), \lambda_{3}^{t, g}(X)<1 / 2$, as observing 2 would induce 1 and 3 to also vote $y$ in non-anchor signals. Thus, it must also hold that: (i) $t=(y, y, y)$ for all $t \notin A n(\pi)$ and (ii) $s_{2}=x$ for all $s \in A n(\pi)$. This implies that for any possible quota $k=1,2,3, V_{k}^{\pi}(g)=V^{p}=2 / 3$, so the maximum value of a straightforward communication strategy would be equal to the value of the optimal public communication strategy.

In contrast, the non-straightforward communication strategy $\pi^{\prime}$ above can improve on this value since:

$$
\operatorname{supp}\left(\sigma^{g}\right)=\left\{\left(\frac{1}{2}, 1, \frac{1}{2}\right),\left(0,0, \frac{1}{2}\right),\left(\frac{1}{2}, 0,0\right)\right\}
$$

and $V_{1}^{\pi}(g)=1$, which is clearly optimal as it is the maximal possible value. Here it is important to note that under $\pi^{\prime}$ after observing the same message $(x)$, receiver 2 votes for $x$ in some signals and for $y$ in others. ${ }^{6}$

A naive extension of straightforwardness to the network setting would suggest that strategies send recommendations to neighborhoods rather than individual receivers, where a receiver

\footnotetext{
${ }^{5}$ This example is close in spirit to Examples 1-3 in Galperti and Perego (2020). However, they demonstrate that not every strategy can be expressed with an alternative straightforward strategy with the same value under their setup, while our example illustrates the fact that under our setup an optimal communication strategy does not always have a corresponding equal-valued straightforward communication strategy.

${ }^{6}$ Proposition 3.16 in the next section shows that in fact the structure of the communcation strategy $\pi^{\prime}$ is optimal and results in the optimal value which can be achieved in any star network.
} 
votes for $x$ if he observes only $x$ in his information neighborhood and votes for $y$ otherwise. Unfortunately, this is also not a viable option as shown in Example 3.7 below.

The process of finding the optimal communication strategy under the empty network is additionally simplified by the fact that restricting attention to anonymous communication strategies is without loss of generality (Kerman et al., 2020). For a general network structure it follows trivially that assuming anonymity is no longer without loss.

\subsubsection{Truth-Telling in the Sender's Preferred State}

In the standard single-receiver case, as well as most multiple-receiver models, telling the truth to the receiver(s) with probability 1 in the sender's preferred state $(X)$ is optimal. This common result ceases to hold in general in the current setup, and this is illustrated by Example 3.7. To help with the analysis we introduce a lemma, which establishes that the maximum number of messages a communication strategy requires to achieve the optimal value is at most equal to its number of anchors plus one.

Lemma 3.6. Let $g \in G(N)$ and $\pi \in \Pi$. There exists $\pi^{\prime} \in \Pi$ with $\left|A n\left(\pi^{\prime}\right)\right|=|A n(\pi)|$ such that for each $i \in N$ it holds that $\left|S_{i}^{\pi^{\prime}}\right| \leq\left|A n\left(\pi^{\prime}\right)\right|+1$ and $V_{k}^{\pi^{\prime}}(g)=V_{k}^{\pi}(g)$.

Example 3.7. Let $|N|=6, \lambda^{0}(X)=1 / 3$, and $k=4$. Consider the following network $g$ and the communication strategy $\pi$ which has one anchor and sends $x$ to all receivers with probability 1 in state $X$.

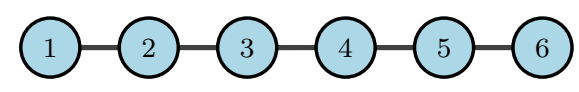

\begin{tabular}{c|cc}
$\pi$ & $\omega=X$ & $\omega=Y$ \\
\hline$(x, x, x, x, x, x)$ & 1 & 0 \\
$(y, x, x, x, x, x)$ & 0 & $\frac{1}{4}$ \\
$(x, x, x, x, x, y)$ & 0 & $\frac{1}{4}$ \\
$(y, y, y, y, y, y)$ & 0 & $\frac{1}{2}$
\end{tabular}

The value of $\pi$ is given by $V_{4}^{\pi}(g)=2 / 3$. This is equal to $V^{p}$, the value of the optimal public communication strategy.

Now consider communication strategy $\pi^{\prime}$, which has two anchor signals, where the support of the distribution of beliefs under $\pi$ is supp $\left(\sigma^{g}\right)$. The value is $V_{4}^{\pi^{\prime}}(g)=1 / 3 \cdot 1+2 / 3 \cdot 3 / 4=$ $5 / 6>2 / 3$, which shows that the sender can improve upon $\pi$ by garbling information in state $X .^{7}$

\footnotetext{
${ }^{7}$ Note that $\pi^{\prime}$ is optimal on $g$ (as implied by Proposition 3.8 in the next section). The same value could have been achieved with at most three messages (cf. Lemma 3.6) by sending a different message in the signal which does not implement $x$. However, it is possible to create this signal such that it is distinguished from both anchors by all nodes with only two messages.
} 


\begin{tabular}{c|cc}
$\pi^{\prime}$ & $\omega=X$ & $\omega=Y$ \\
\hline$(x, x, x, x, x, x)$ & $\frac{1}{2}$ & 0 \\
$(y, y, y, y, y, y)$ & $\frac{1}{2}$ & 0 \\
$(x, x, x, y, y, y)$ & 0 & $\frac{1}{4}$ \\
$(y, x, x, x, x, x)$ & 0 & $\frac{1}{4}$ \\
$(y, y, y, y, y, x)$ & 0 & $\frac{1}{4}$ \\
$(y, x, y, x, y, x)$ & 0 & $\frac{1}{4}$
\end{tabular}

$$
\begin{aligned}
\operatorname{supp}\left(\sigma^{g}\right)= & \left\{\left(\frac{1}{2}, \frac{1}{2}, \frac{1}{2}, \frac{1}{2}, \frac{1}{2}, \frac{1}{2}\right),\left(\frac{1}{2}, \frac{1}{2}, \frac{1}{2}, \frac{1}{2}, \frac{1}{2}, \frac{1}{2}\right),\right. \\
& \left(\frac{1}{2}, \frac{1}{2}, 0,0, \frac{1}{2}, \frac{1}{2}\right),\left(0,0, \frac{1}{2}, \frac{1}{2}, \frac{1}{2}, \frac{1}{2}\right), \\
& \left.\left(\frac{1}{2}, \frac{1}{2}, \frac{1}{2}, \frac{1}{2}, 0,0\right),(0,0,0,0,0,0)\right\} .
\end{aligned}
$$

It is important that $V_{4}^{\pi^{\prime}}(g)$ cannot be achieved in a strategy with only one anchor. To prove this, assume the contrary. By Lemma 3.6, there exists $\pi^{\prime \prime} \in \Pi$ such that for every $i \in N$ it holds that $\left|S_{i}^{\pi^{\prime \prime}}\right|=2$ and $V_{4}^{\pi^{\prime \prime}}(g)=V_{4}^{\pi^{\prime}}(g)=5 / 6$. Assume without loss of generality that for each $i \in N, S_{i}^{\pi^{\prime \prime}}=\{x, y\}$. As truth-telling in state $X$ requires, let $\mathbf{x}=(x, \ldots, x)$ be the anchor and let $\pi^{\prime \prime}(\mathbf{x} \mid X)=1$. Let $t \in S^{\pi^{\prime \prime}}$ with $t \neq \mathbf{x}$. If for any $i \in N$ it holds that $t_{i} \neq x$, then for any $j \in \bar{N}_{i}(g)$ we have $\alpha_{j}^{\pi^{\prime \prime}, g}\left(t_{j}(g)\right)=y$ and thus, if $i \in\{2,3,4,5\}$, then $\alpha^{\pi^{\prime \prime}, g}(t)=y$, i.e. $t$ does not implement $x$. Thus, in signals that implement $x$, only receivers 1 and 6 can observe a message different than $x$. In this case, an optimal communication strategy is $\pi$ given above, which has value $2 / 3$, contradicting that $V_{4}^{\pi^{\prime \prime}}(g)=5 / 6$. Therefore, truth-telling in state $X$ is not optimal in general.

Recall the naive straightforwardness extension in the previous section; it is not violated by $\pi$. Moreover, $\pi$ is optimal among communication strategies which satisfy this definition. Since we can improve upon $\pi$ with the "non-naively straightforward" $\pi^{\prime}$, it follows that the naive extension is not optimal either. The feature which makes $\pi^{\prime}$ better than $\pi$ is that apart from the different personal messages within signals, it uses the differences in the messages between the two anchors to create more variation in the strategy.

\subsection{General Observations}

As shown, many of the usual simplifying assumptions in the empty network Bayesian persuasion model do not generally extend to non-empty networks. Nevertheless, there are optimality results which can be recovered and they are the subject of this section.

\subsubsection{The Network Does not Benefit the Sender}

We start by showing that having a network structure cannot be strictly beneficial for the sender. More precisely, for any communication strategy the optimal value under the empty network $g_{0}$ is at least as good as the optimal value under any other network $g$ with the same number of nodes. This insight was already hinted at in Example 3.1. The simple logic behind 
this observation is that in a non-empty network the vote of every node is determined not by the individual messages of every receiver but by his whole neighborhood. However, the information a receiver gathers from a communication strategy in a non-empty network can be replicated in an empty network of the same size.

Proposition 3.8. Let $g \in G(N)$. For any $\pi \in \Pi$ it holds that $V_{k}^{\pi}(g) \leq V_{k}^{n}$.

Intuitively, the reason the empty network value weakly dominates the value of any other network with the same number of nodes is that the sender can target at least as many winning coalitions under the empty network as under any non-empty network. However, this might not hold when comparing two non-empty networks, as shown in Section 3.6.

\subsubsection{Networks with Isolated Nodes}

This section shows that with sufficiently many isolated nodes the network has no effect on what the sender can achieve. In particular, if $k \geq n / 2$ and if the number of singletons in a network is at least $k$, then the sender can achieve the empty network optimal value, irrespective of the connections between the remaining nodes.

Example 3.9. Let $|N|=9, \lambda^{0}(X)=1 / 3$, and $k=5$. Consider network $g$ below, the communication strategy $\pi$ and the support of its induced distribution. Note that $2 k=10>9=n$, so the singleton nodes are equal to $k$ and are a simple majority.
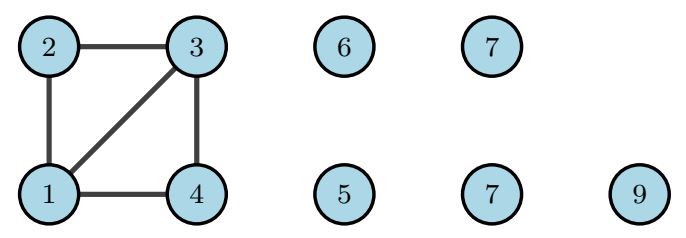

\begin{tabular}{c|cc}
$\pi$ & $\omega=X$ & $\omega=Y$ \\
\hline$(x, x, x, x, x, x, x, x, x)$ & 1 & 0 \\
$(y, y, y, y, x, x, x, x, x)$ & 0 & $\frac{4}{10}$ \\
$(x, x, x, x, x, y, y, y, y)$ & 0 & $\frac{1}{10}$ \\
$(x, x, x, x, y, x, y, y, y)$ & 0 & $\frac{1}{10}$ \\
$(x, x, x, x, y, y, x, y, y)$ & 0 & $\frac{1}{10}$ \\
$(x, x, x, x, y, y, y, x, y)$ & 0 & $\frac{1}{10}$ \\
$(x, x, x, x, y, y, y, y, x)$ & 0 & $\frac{1}{10}$ \\
$(y, y, y, y, y, y, y, y, y)$ & 0 & $\frac{1}{10}$
\end{tabular}

$$
\begin{aligned}
\operatorname{supp}\left(\sigma^{g}\right)= & \left\{\left(\frac{1}{2}, \frac{1}{2}, \frac{1}{2}, \frac{1}{2}, \frac{1}{2}, \frac{1}{2}, \frac{1}{2}, \frac{1}{2}, \frac{1}{2}\right),\left(0,0,0,0, \frac{1}{2}, \frac{1}{2}, \frac{1}{2}, \frac{1}{2}, \frac{1}{2}\right),\left(\frac{1}{2}, \frac{1}{2}, \frac{1}{2}, \frac{1}{2}, \frac{1}{2}, 0,0,0,0\right),\right. \\
& \left(\frac{1}{2}, \frac{1}{2}, \frac{1}{2}, \frac{1}{2}, 0, \frac{1}{2}, 0,0,0\right),\left(\frac{1}{2}, \frac{1}{2}, \frac{1}{2}, \frac{1}{2}, 0,0, \frac{1}{2}, 0,0\right),\left(\frac{1}{2}, \frac{1}{2}, \frac{1}{2}, \frac{1}{2}, 0,0,0, \frac{1}{2}, 0\right), \\
& \left.\left(\frac{1}{2}, \frac{1}{2}, \frac{1}{2}, \frac{1}{2}, 0,0,0,0, \frac{1}{2}\right),(0,0,0,0,0,0,0,0,0)\right\} .
\end{aligned}
$$


The value is $V_{5}^{\pi}(g)=1 / 3 \cdot 1+2 / 3 \cdot 9 / 10=14 / 15=V_{5}^{9}$, the value for persuading $k=5$ out of $n=9$ in an empty network. Observe that this value can be achieved irrespective of the connections between nodes 1-4, as these nodes are always treated identically.

Proposition 3.10. Let $g \in G(N)$ and $\left|\left\{i \in N: \delta_{i}^{g}=0\right\}\right| \geq k$. If $k \geq n / 2$, then there exists $\pi \in \Pi$ such that $V_{k}^{\pi}(g)=V_{k}^{n}$.

\subsubsection{Networks Consisting of Complete Components}

If two nodes $i$ and $j$ in a network $g$ have exactly the same neighborhood, $\bar{N}_{i}(g)=\bar{N}_{j}(g)$, then the sets of signals they consider possible under any communication strategy $\pi$ are the same, i.e. $A_{i}^{\pi}(g, s)=A_{j}^{\pi}(g, s)$. This implies that they always have the same posterior belief and vote for the same alternative, i.e. for every $s \in S^{\pi}$ it holds that $\alpha_{i}^{\pi, g}\left(s_{i}(g)\right)=\alpha_{j}^{\pi, g}\left(s_{j}(g)\right)$. Therefore, in such cases we can restrict attention to communication strategies which send the same message to $i$ and $j$. In particular, the information that the sender wants to provide to the neighbours of $i$ and $j$ by sending different messages to $i$ and $j$ can also be provided to the neighbours by sending the same message to $i$ and $j$ within the same signal. Note that in this case $i$ and $j$ can still observe different messages between signals. This logic is formalized in Proposition 3.11, which is particularly relevant for members of a complete component because they always share the same information neighorhood.

Proposition 3.11. Let $\pi \in \Pi$ and let $g \in G(N)$ and $i, j \in N$ be such that $\bar{N}_{i}(g)=\bar{N}_{j}(g)$. Then there exists $\pi^{\prime} \in \Pi$ such that for any $s \in S^{\pi^{\prime}}, s_{i}=s_{j}$ and $V_{k}^{\pi^{\prime}}(g)=V_{k}^{\pi}(g)$.

As every receiver in a complete network has the same information neighborhood, an immediate corollary to Proposition 3.11 follows.

Corollary 3.12. Let $g \in G(N)$ be a complete network. Then there exists an optimal $\pi \in \Pi$ such that $V_{k}^{\pi}(g)=V^{p}$.

So, the optimal communication strategy in a complete network is public as it is optimal for the sender to treat all receivers within a signal uniformly by sending them same message.

Proposition 3.11 naturally extends to networks of disjoint complete components. Treating all nodes within a component uniformly in every signal makes this setup similar to an empty network with fewer nodes where every node has a different number of votes.

Example 3.13. Let $|N|=9, \lambda^{0}(X)=1 / 3$, and $k=5$. Consider the following network $g$ with three complete components and the communication strategy $\pi \in \Pi$ : 

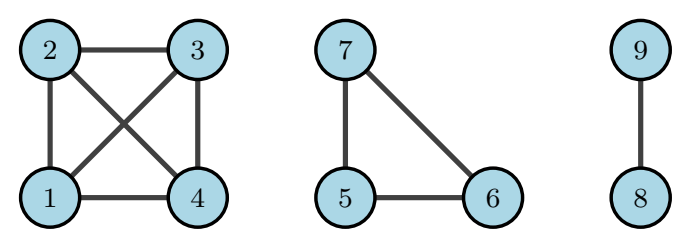

\begin{tabular}{c|cc}
$\pi$ & $\omega=X$ & $\omega=Y$ \\
\hline$(x, x, x, x, x, x, x, x, x)$ & 1 & 0 \\
$(y, y, y, y, x, x, x, x, x)$ & 0 & $\frac{1}{4}$ \\
$(x, x, x, x, y, y, y, x, x)$ & 0 & $\frac{1}{4}$ \\
$(x, x, x, x, x, x, x, y, y)$ & 0 & $\frac{1}{4}$ \\
$(y, y, y, y, y, y, y, y, y)$ & 0 & $\frac{1}{4}$
\end{tabular}

Here, selecting any component to receive a y message leaves enough nodes (5, 6, or 7) to implement $x$. This results in:

$$
\begin{aligned}
\operatorname{supp}\left(\sigma^{g}\right)= & \left\{\left(\frac{1}{2}, \frac{1}{2}, \frac{1}{2}, \frac{1}{2}, \frac{1}{2}, \frac{1}{2}, \frac{1}{2}, \frac{1}{2}, \frac{1}{2}\right),\left(0,0,0,0, \frac{1}{2}, \frac{1}{2}, \frac{1}{2}, \frac{1}{2}, \frac{1}{2}\right),\left(\frac{1}{2}, \frac{1}{2}, \frac{1}{2}, \frac{1}{2}, 0,0,0, \frac{1}{2}, \frac{1}{2}\right),\right. \\
& \left.\left(\frac{1}{2}, \frac{1}{2}, \frac{1}{2}, \frac{1}{2}, \frac{1}{2}, \frac{1}{2}, \frac{1}{2}, 0,0\right),(0,0,0,0,0,0,0,0,0)\right\} .
\end{aligned}
$$

In each signal except $\mathbf{y}, \pi$ persuades two out of three components, which suffices to implement $x$. It it straightforward to check that all signals except $\mathbf{y}$ implement $x$. The value is $V_{5}^{\pi}(g)=$ $1 / 3 \cdot 1+2 / 3 \cdot 3 / 4=5 / 6=V_{2}^{3}$, the value for persuading $k=2$ out of $n=3$ nodes in the empty network and it is clear that no higher value can be achieved.

However, this is not necessarily the case if selecting a different number of components in different signals can still fulfil the quota, as this allows for much more variation. For example, when nodes 5, 6,7 are singletons strategy $\pi^{\prime}$ can be applied.
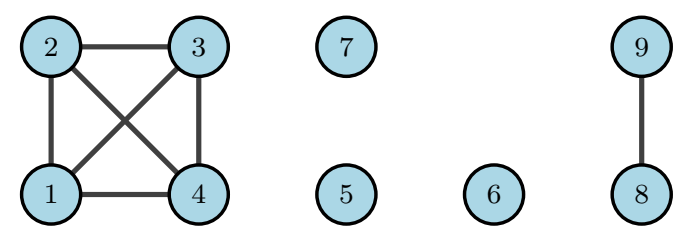

\begin{tabular}{c|cc}
$\pi^{\prime}$ & $\omega=X$ & $\omega=Y$ \\
\hline$(x, x, x, x, x, x, x, x, x)$ & 1 & 0 \\
$(y, y, y, y, x, x, x, x, x)$ & 0 & $\frac{1}{3}$ \\
$(x, x, x, x, y, x, y, y, y)$ & 0 & $\frac{1}{6}$ \\
$(x, x, x, x, x, y, y, y, y)$ & 0 & $\frac{1}{6}$ \\
$(x, x, x, x, y, y, x, y, y)$ & 0 & $\frac{1}{6}$ \\
$(y, y, y, y, y, y, y, y, y)$ & 0 & $\frac{1}{6}$
\end{tabular}

In this case the support of $\pi^{\prime}$ is:

$$
\begin{aligned}
\operatorname{supp}\left(\sigma^{g}\right)= & \left\{\left(\frac{1}{2}, \frac{1}{2}, \frac{1}{2}, \frac{1}{2}, \frac{1}{2}, \frac{1}{2}, \frac{1}{2}, \frac{3}{5}, \frac{3}{5}\right),\left(0,0,0,0, \frac{1}{2}, \frac{1}{2}, \frac{1}{2}, \frac{3}{5}, \frac{3}{5}\right),\left(\frac{1}{2}, \frac{1}{2}, \frac{1}{2}, \frac{1}{2}, 0, \frac{1}{2}, 0,0,0\right),\right. \\
& \left.\left(\frac{1}{2}, \frac{1}{2}, \frac{1}{2}, \frac{1}{2}, \frac{1}{2}, 0,0,0,0\right),\left(\frac{1}{2}, \frac{1}{2}, \frac{1}{2}, \frac{1}{2}, 0,0, \frac{1}{2}, 0,0\right),(0,0,0,0,0,0,0,0,0)\right\}
\end{aligned}
$$

The value is $V_{5}^{\pi^{\prime}}(g)=1 / 3 \cdot 1+2 / 3 \cdot 5 / 6=8 / 9>5 / 6$.

To formalize the logic of the example, given a network $g$, let $C(g)$ be the set of all components of $g$. Let $\mathfrak{C}^{q}$ be the set of all subsets of components, where each subset has cardinality $q$. That is, $\mathfrak{C}^{q}=\left\{\mathcal{C}^{\prime} \subseteq C(g):\left|\mathcal{C}^{\prime}\right|=q\right\}$.

A network $g \in G(N)$ is connected if for any $i, j \in N$ there is a path between $i$ and $j$. 
Proposition 3.14. Let $g \in G(N)$ be a network consisting of $\ell$ complete components of respective sizes $c_{1}, c_{2}, \ldots, c_{\ell} \in C(g)$. If $q \in \mathbb{N}$ is such that for each $\mathcal{C}^{\prime} \in \mathfrak{C}^{q}$ it holds that $\sum_{C \in \mathcal{C}^{\prime}}|C| \geq k$, then there exists an optimal $\pi \in \Pi$ such that $V_{k}^{\pi}(g)=V_{q}^{\ell}$.

So, if a network consists of $\ell$ complete disjoint components and combining the same number of components $q$ fulfils the quota, it is possible to construct a strategy with value equal to the optimal value of persuading $q$ out of $\ell$ agents. This follows directly from the uniform treatment of all nodes within the same component as per Proposition 3.11.

\subsection{Optimality in Specific Network Structures}

This section provides optimal strategies for networks which frequently appear in the literature in different contexts, cf. in social communication in financial markets (Bushman and Indjejikian, 1995; Colla and Mele, 2010) or in network formation models (Bala and Goyal, 2000). Every subsection deals with different network types and starts with an example conveying the intuition which drives the more general result and the main mechanisms to construct the optimal strategies. This is followed by a formal statement of the result.

\subsubsection{Star Networks}

A network $g \in G(N)$ is a star if there exists $j \in N$ such that for any $i \in N$ with $i \neq j$ it holds that $g_{i j}=1$ and for any $\ell \in N$ with $\ell \neq j$ it holds that $g_{i \ell}=0$. The star presents a situation in which the optimal value under the empty network cannot be achieved.

Before proceeding to the formal statement about star networks, consider the example below, which bears similarity to Example 3.5. The communication strategy employed keeps the center's message the same across all signals which implement $x$ while varying the periphery nodes' messages in the same way as in the empty network and thus achieves value equal to $V_{k}^{n-1}$. This is less than $V_{k}^{n}$ and so the optimal value in the empty network with private communication is not achieved in this case.

Example 3.15. Let $|N|=5, \lambda^{0}(X)=1 / 3$, and $k=3$. Consider the following network $g$, the communication strategy $\pi$, and its support.

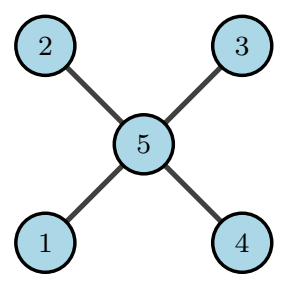

\begin{tabular}{c|cc}
$\pi$ & $\omega=X$ & $\omega=Y$ \\
\hline$(x, x, x, x, x)$ & 1 & 0 \\
$(y, x, x, x, x)$ & 0 & $\frac{1}{6}$ \\
$(x, y, x, x, x)$ & 0 & $\frac{1}{6}$ \\
$(x, x, y, x, x)$ & 0 & $\frac{1}{6}$ \\
$(x, x, x, y, x)$ & 0 & $\frac{1}{6}$ \\
$(y, y, y, y, y)$ & 0 & $\frac{1}{3}$
\end{tabular}




$$
\begin{aligned}
\operatorname{supp}\left(\sigma^{g}\right)= & \left\{\left(\frac{1}{2}, \frac{1}{2}, \frac{1}{2}, \frac{1}{2}, 1\right),\left(0, \frac{1}{2}, \frac{1}{2}, \frac{1}{2}, 0\right),\left(\frac{1}{2}, 0, \frac{1}{2}, \frac{1}{2}, 0\right),\left(\frac{1}{2}, \frac{1}{2}, 0, \frac{1}{2}, 0\right),\left(\frac{1}{2}, \frac{1}{2}, \frac{1}{2}, 0,0\right)\right. \\
& (0,0,0,0,0)\}
\end{aligned}
$$

The value is $V_{3}^{\pi}(g)=1 / 3 \cdot 1+2 / 3 \cdot 2 / 3=7 / 9=V_{3}^{4}$.

Proposition 3.16. Let $g \in G(N)$ be a star and let $k<n$. Then there exists an optimal $\pi \in \Pi$ such that $V_{k}^{\pi}(g)=V_{k}^{n-1}$.

In the optimal communication strategy specified in the proof of Proposition 3.16 the center node observes the same message in all signals which implement $x$. It is possible to extrapolate this logic to all networks which have nodes that are connected to all other nodes. In the limit case (the complete network), this corresponds to sending the same messages to all receivers within a signal for every signal, i.e. a public communication strategy. ${ }^{8}$

\subsubsection{Regular Networks}

A network $g$ is regular if for any $i, j \in N$ it holds that $\delta_{i}^{g}=\delta_{j}^{g}=\delta$. Regular networks are a natural extension of the situation described by Theorem 2.8 and Corollary 3.12, as the empty network is also regular. A regular connected network with $\delta=2$ is a circle.

Example 3.17. Let $|N|=9, \lambda^{0}(X)=1 / 3$, and $k=6$. Consider the following network $g$, the communication strategy $\pi$ and its support:

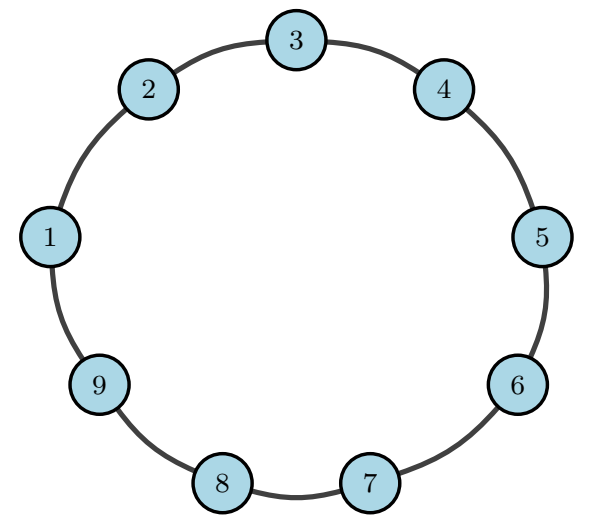

\begin{tabular}{c|cc}
$\pi$ & $\omega=X$ & $\omega=Y$ \\
\hline$(x, x, x, x, x, x, x, x, x)$ & 1 & 0 \\
$(y, x, x, x, x, x, x, x, x)$ & 0 & $\frac{1}{12}$ \\
$(x, y, x, x, x, x, x, x, x)$ & 0 & $\frac{1}{12}$ \\
$(x, x, y, x, x, x, x, x, x)$ & 0 & $\frac{1}{12}$ \\
$(x, x, x, y, x, x, x, x, x)$ & 0 & $\frac{1}{12}$ \\
$(x, x, x, x, y, x, x, x, x)$ & 0 & $\frac{1}{12}$ \\
$(x, x, x, x, x, y, x, x, x)$ & 0 & $\frac{1}{12}$ \\
$(x, x, x, x, x, x, y, x, x)$ & 0 & $\frac{1}{12}$ \\
$(x, x, x, x, x, x, x, y, x)$ & 0 & $\frac{1}{12}$ \\
$(x, x, x, x, x, x, x, x, y)$ & 0 & $\frac{1}{12}$ \\
$(y, y, y, y, y, y, y, y, y)$ & 0 & $\frac{1}{4}$
\end{tabular}

\footnotetext{
${ }^{8}$ Similarly, when $n=k$ in a star, optimal communication is public.
} 


$$
\begin{aligned}
\operatorname{supp}\left(\sigma^{g}\right)=\{ & \left(\frac{1}{2}, \frac{1}{2}, \frac{1}{2}, \frac{1}{2}, \frac{1}{2}, \frac{1}{2}, \frac{1}{2}, \frac{1}{2}, \frac{1}{2}\right),\left(0,0, \frac{1}{2}, \frac{1}{2}, \frac{1}{2}, \frac{1}{2}, \frac{1}{2}, \frac{1}{2}, 0\right), \\
& \left(0,0,0, \frac{1}{2}, \frac{1}{2}, \frac{1}{2}, \frac{1}{2}, \frac{1}{2}, \frac{1}{2}\right),\left(\frac{1}{2}, 0,0,0, \frac{1}{2}, \frac{1}{2}, \frac{1}{2}, \frac{1}{2}, \frac{1}{2}\right),\left(\frac{1}{2}, \frac{1}{2}, 0,0,0, \frac{1}{2}, \frac{1}{2}, \frac{1}{2}, \frac{1}{2}\right), \\
& \left(\frac{1}{2}, \frac{1}{2}, \frac{1}{2}, 0,0,0, \frac{1}{2}, \frac{1}{2}, \frac{1}{2}\right),\left(\frac{1}{2}, \frac{1}{2}, \frac{1}{2}, \frac{1}{2}, 0,0,0, \frac{1}{2}, \frac{1}{2}\right),\left(\frac{1}{2}, \frac{1}{2}, \frac{1}{2}, \frac{1}{2}, \frac{1}{2}, 0,0,0, \frac{1}{2}\right), \\
& \left.\left(\frac{1}{2}, \frac{1}{2}, \frac{1}{2}, \frac{1}{2}, \frac{1}{2}, \frac{1}{2}, 0,0,0\right),\left(0, \frac{1}{2}, \frac{1}{2}, \frac{1}{2}, \frac{1}{2}, \frac{1}{2}, \frac{1}{2}, 0,0\right),(0,0,0,0,0,0,0,0,0)\right\} .
\end{aligned}
$$

Each receiver observes $x$ with probability 1 in state $X$. Hence, sending $y$ to a receiver in state $Y$ implies that 3 receivers vote for $y$. That is, for any $i \in N$ and $s \in S^{\pi}$ if $s_{i}=y$ and $j \in \bar{N}_{i}(g)$, then $\lambda_{j}^{s}(X)=0$. So, in a signal implementing $x$ in state $Y$ exactly 6 receivers are persuaded and $V_{6}^{\pi}(g)=1 / 3 \cdot 1+2 / 3 \cdot 9 / 12=5 / 6=V_{6}^{9}$.

Proposition 3.18. Let $g \in G(N)$ be a circle and let $k<n-2$. Then there exists $\pi \in \Pi$ such that $V_{k}^{\pi}(g)=V_{k}^{n}$.

Proposition 3.18 formalizes the observation in Example 3.17 and establishes that if the quota is less than $n-2$, the optimal value under the empty network can be achieved on a circle.

A network which combines many properties of circle and star networks is the wheel: formally, a network $g \in G(N)$ is a wheel if there exists $j \in N$ such that for any $i \in N$ with $i \neq j$ it holds that: (i) $g_{i j}=1$, (ii) $\delta_{i}^{g}=3$, and (iii) for any $\ell \in N, \ell \neq j$, and $\ell \neq i$ there is a path between $i$ and $\ell$ which does not include $j$.

The reasoning behind sending the same message to the center node in all signals which implement $x$ used for the star also applies to the wheel. In this case, the sender can treat the network as if it is a circle to achieve the optimal value provided that the quota is less than $n-3$. This observation is stated as a corollary to Propositions 3.16 and 3.18.

Corollary 3.19. Let $g \in G(N)$ be a wheel and let $k<n-3$. Then there exists an optimal $\pi \in \Pi$ such that $V_{k}^{\pi}(g)=V_{k}^{n-1}$.

Proposition 3.20 shows that if $k=n-1-\delta$ the logic used for the circle can be applied to every regular network to obtain the optimal value.

Proposition 3.20. Let $g \in G(N)$ be a regular network with degree $\delta$ and let $k=n-1-\delta$. Then there exists $\pi \in \Pi$ such that $V_{k}^{\pi}(g)=V_{k}^{n}$.

The following example illustrates that extending the reasoning in the proposition to $k<$ $n-1-\delta$ is a non-trivial task. 
Example 3.21. Consider the following regular networks $g_{1}, g_{2}$ and $g_{3}$, all with degree 3 .

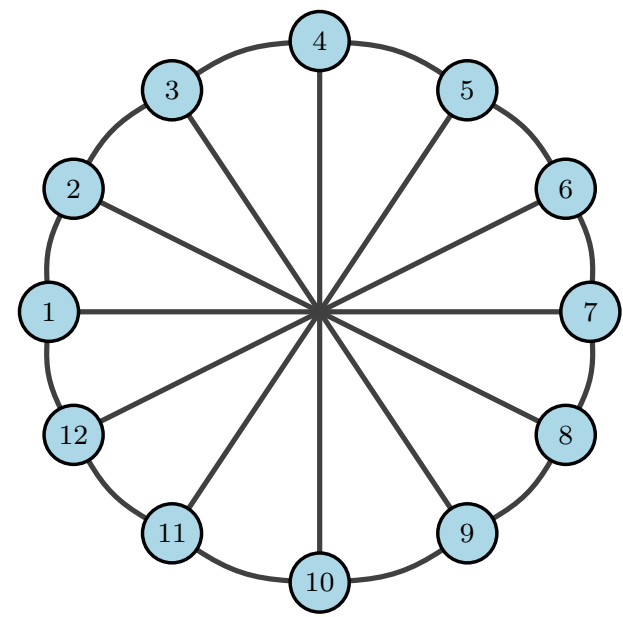

Network $g_{1}$

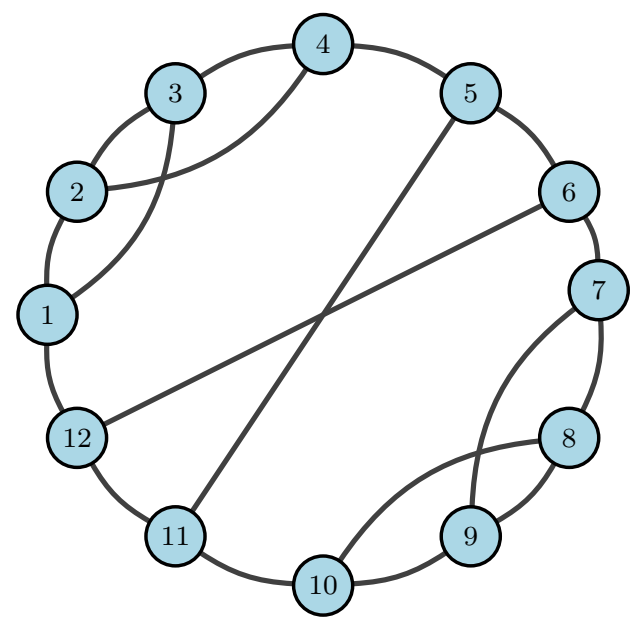

Network $g_{2}$
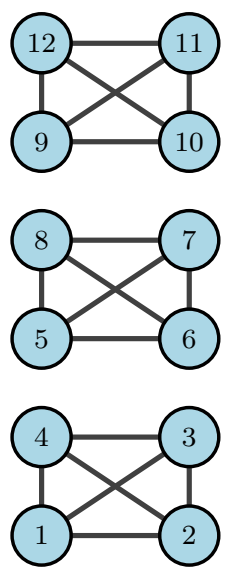

Network $g_{3}$

Let $\lambda^{0}(X)=1 / 3$. By Proposition 3.20, the optimal value under the empty network can be achieved in all three networks for $k=8$. However, if $k=6$ we can easily construct a communication strategy with value equal to $V_{6}^{12}=V_{1}^{2}=1$ for $g_{1}$ and $g_{2}$ (similar to the construction in Example 3.17), but not for $g_{3}$. Because the network is disconnected and each component is complete, persuading exactly 6 receivers is impossible due to Proposition 3.11 as receivers in a complete component all vote for the same alternative. In particular, any signal that implements $x$ has at least 8 receivers who vote for $x$, implying that the value 1 cannot be achieved.

Decreasing the quota to $k=4$, it is once again easy to construct optimal strategies for $g_{1}$ and $g_{3}$, but an optimal strategy for $g_{2}$ will clearly have a different structure.

It is important to note that the conditions in Proposition 3.18 and 3.20 are sufficient, but not necessary. The next example provides a network which satisfies neither of them but the optimal communication strategy on it still achieves the maximum value.

Example 3.22. Let $|N|=4, \lambda^{0}(X)=1 / 3$. Consider the following network $g$ and the communication strategies $\pi$ for $k=2$ and $\pi^{\prime}$ for $k=3$ with corresponding supports. 


\begin{tabular}{c|cc}
$\pi$ & $\omega=X$ & $\omega=Y$ \\
\hline$(x, x, y, y)$ & $\frac{1}{4}$ & 0 \\
$(x, y, y, x)$ & $\frac{1}{4}$ & 0 \\
$(y, y, x, x)$ & $\frac{1}{4}$ & 0 \\
$(y, x, x, y)$ & $\frac{1}{4}$ & 0 \\
$(y, y, y, x)$ & 0 & $\frac{1}{8}$ \\
$(y, y, x, y)$ & 0 & $\frac{1}{8}$ \\
$(y, x, y, y)$ & 0 & $\frac{1}{8}$ \\
$(x, y, y, y)$ & 0 & $\frac{1}{8}$ \\
$(x, x, x, y)$ & 0 & $\frac{1}{8}$ \\
$(x, x, y, x)$ & 0 & $\frac{1}{8}$ \\
$(x, y, x, x)$ & 0 & $\frac{1}{8}$ \\
$(y, x, x, x)$ & 0 & $\frac{1}{8}$
\end{tabular}

$$
\begin{array}{cc|cc}
\operatorname{supp}\left(\sigma^{g}\right)=\left\{\left(\frac{1}{2}, \frac{1}{2}, \frac{1}{2}, \frac{1}{2}\right),\right. & (y, y, x, x) & \frac{1}{6} & 0 \\
\left.\left(\frac{1}{2}, 0, \frac{1}{2}, 0\right),\left(0, \frac{1}{2}, 0, \frac{1}{2}\right)\right\} & (y, x, y, y) & \frac{1}{6} & 0 \\
& (x, y, y, x) & \frac{1}{6} & 0 \\
& (y, y, y, y) & \frac{1}{6} & 0 \\
& (x, x, x, y) & 0 & \frac{1}{12} \\
& (y, x, x, x) & 0 & \frac{1}{12} \\
& (x, x, y, x) & 0 & \frac{1}{12} \\
\operatorname{supp}\left(\sigma^{g}\right)=\left\{\left(\frac{1}{2}, \frac{1}{2}, \frac{1}{2}, \frac{1}{2}\right),\right. & (y, y, y, x) & 0 & \frac{1}{12} \\
\left(\frac{1}{2}, \frac{1}{2}, \frac{1}{2}, 0\right),\left(0, \frac{1}{2}, \frac{1}{2}, \frac{1}{2}\right), & (x, y, y, y) & 0 & \frac{1}{12} \\
\left.\left(\frac{1}{2}, \frac{1}{2}, 0, \frac{1}{2}\right),\left(\frac{1}{2}, 0, \frac{1}{2}, \frac{1}{2}\right)\right\} & (y, y, x, y) & 0 & \frac{1}{12} \\
& (y, x, y, y) & 0 & \frac{1}{12} \\
& (x, y, x, y) & 0 & \frac{1}{3}
\end{array}
$$

where in the first support the first four signals in $\pi$ produce the first posterior, the odd-numbered remaining signals have the second one and the even-numbered remaining signals have the third posterior. As all signals in $\pi$ implement $x$, the value is $V_{2}^{\pi}(g)=1=V_{2}^{4}$, while for $\pi^{\prime}$ it is $V_{3}^{\pi^{\prime}}(g)=7 / 9=V_{3}^{4}$. Following the same logic as in Example 3.7 it is possible to see that in both cases the values cannot be achieved by simpler strategies, i.e. ones with fewer total signals or fewer anchors.

As a receiver obtains information not from a message but from the difference in his neighbors' messages, the underlying structures also cannot be readily extrapolated to optimal strategies for the general case of $k=n-2$ or $k=n-1$ on a circle, as such an extrapolation would require using more than two messages.

\subsubsection{Irregular Networks}

Moving away from regular networks, it is possible to derive intuitive results for irregular networks that have some symmetry in the sense that the network can be partitioned into sets of receivers that vote for $y$ after a signal realization.

Example 3.23. Let $|N|=36, \lambda^{0}(X)=1 / 3$, and $k=27$. Consider network $g$ below. 


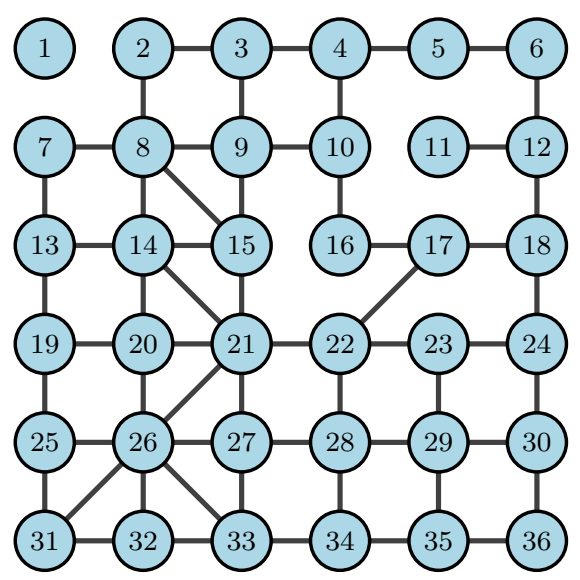

\begin{tabular}{c|cc}
$\pi$ & $\omega=X$ & $\omega=Y$ \\
\hline $\mathbf{x}$ & 1 & 0 \\
$s^{1}$ & 0 & $\frac{1}{6}$ \\
$s^{2}$ & 0 & $\frac{1}{6}$ \\
$s^{3}$ & 0 & $\frac{1}{6}$ \\
$s^{4}$ & 0 & $\frac{1}{6}$ \\
$\mathbf{y}$ & 0 & $\frac{1}{3}$
\end{tabular}

Notice that there are no links between nodes 15 and 16, and 16 and 22. Further, it is important that while the network shows some symmetry, it is not regular as there are nodes with degrees from 0 to \%. Additionally, exactly $1 / 4$ of the network can be covered (in the sense that a quarter of receivers vote for $y$ ) by sending a message $y$ to specific agents in one of the quadrants. In particular, sending y to receivers 1, 2, 7, and 8 makes receivers in the $N W$ quadrant observe at least one $y$ in their information neighborhood. Define signals:

(i) $s^{1} \in S: \forall i \in N, s_{i}^{1}=y$ if $i \in\{1,2,7,8\}$ and $s_{i}^{1}=x$ otherwise,

(ii) $s^{2} \in S: \forall i \in N, s_{i}^{2}=y$ if $i \in\{5,12,16\}$ and $s_{i}^{1}=x$ otherwise,

(iii) $s^{3} \in S: \forall i \in N, s_{i}^{3}=y$ if $i \in\{25,26\}$ and $s_{i}^{1}=x$ otherwise,

(iv) $s^{4} \in S: \forall i \in N, s_{i}^{4}=y$ if $i \in\{23,29,35\}$ and $s_{i}^{1}=x$ otherwise.

Consider communication strategy $\pi$ and note that $s^{1}, s^{2}, s^{3}$, and $s^{4}$ make receivers in the $N W$, $N E, S W$, and $S E$ quadrant respectively vote for $y . V_{27}^{\pi}(g)=V_{27}^{36}=V_{3}^{4}=7 / 9$. It is interesting to remark that despite the relatively complicated structure of the network, the communication strategy only consists of six signals.

Proposition 3.24. Let $g \in G(N)$ be a network and let $q \in \mathbb{N}$ be a common factor of $n$ and $k$. If there exist $n / q$ disjoint sets of nodes $O_{1}, \ldots, O_{n / q} \subseteq N$ such that for each $j \in\{1, \ldots, n / q\}$ it holds that $\left|O_{j}\right|=q$ and for each $O_{j} \subseteq N$ there exists $L_{j} \subseteq O_{j}$ with $\cup_{i \in L_{j}} \bar{N}_{i}(g)=O_{j}$, then there exists $\pi \in \Pi$ with $V_{k}^{\pi}(g)=V_{k}^{n}$.

It is important to note that the Proposition 3.24 presents only a sufficient condition. It generalizes the observation from Example 3.23 that if equal-sized parts of the network can observe at least one $y$ message in their information neighborhood in different signals this can be leveraged to get the optimal value, irrespective of regularity. For example, one can easily construct a simple, three-signal strategy to optimally convince precisely half of the voters in the example and achieve $V_{18}^{36}=V_{1}^{2}=1$, i.e. $100 \%$ persuasion rate. Proposition 3.24 also offers 
possibilities for practical application in real life social networks, which usually exhibit high degrees of clustering (Jackson and Rogers, 2007). Finally, Proposition prop:multiples also extends the underlying logic behind Proposition 3.18 and Proposition 3.20 to some irregular networks.

\section{Line Networks}

The line network is a notable case of a network which in some cases can be divided in the way described in Proposition 3.24.

Example 3.25. Let $|N|=9, \lambda^{0}(X)=1 / 3$, and $k=6$. Consider the following network $g$, the communication strategy $\pi \in \Pi$ and its support:

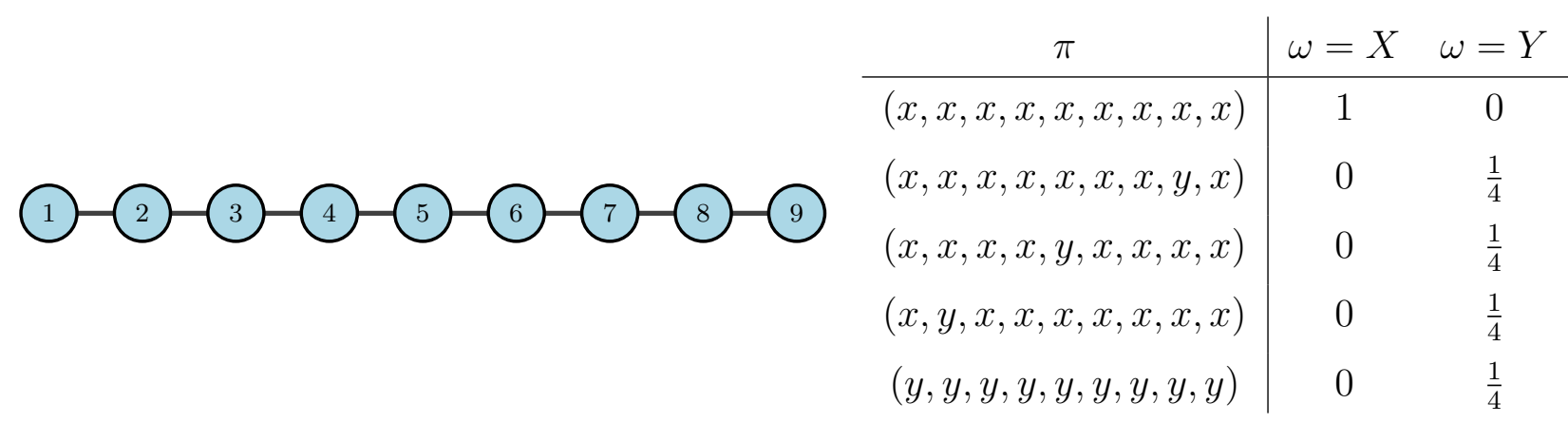

$$
\begin{aligned}
& \operatorname{supp}\left(\sigma^{g}\right)=\left\{\left(\frac{1}{2}, \frac{1}{2}, \frac{1}{2}, \frac{1}{2}, \frac{1}{2}, \frac{1}{2}, \frac{1}{2}, \frac{1}{2}, \frac{1}{2}\right),\left(\frac{1}{2}, \frac{1}{2}, \frac{1}{2}, \frac{1}{2}, \frac{1}{2}, \frac{1}{2}, 0,0,0\right),\left(\frac{1}{2}, \frac{1}{2}, \frac{1}{2}, 0,0,0, \frac{1}{2}, \frac{1}{2}, \frac{1}{2},\right)\right. \text {, } \\
& \left.\left(0,0,0, \frac{1}{2}, \frac{1}{2}, \frac{1}{2}, \frac{1}{2}, \frac{1}{2}, \frac{1}{2}\right),(0,0,0,0,0,0,0,0,0)\right\} \text {. }
\end{aligned}
$$

For any $i \in N$ and $s \in S^{\pi}$ if $s_{i}=y$ and $j \in N_{i}(g)$, then $\lambda_{i}^{s}(X)=\lambda_{j}^{s}(X)=0$. The value is $V_{6}^{\pi}(g)=1 / 3 \cdot 1+2 / 3 \cdot 3 / 4=5 / 6=V_{6}^{9}$. Interestingly, by Proposition 3.24, $\pi$ is a simpler alternative to the communication strategy in Example 3.17. It preserves the value $V_{6}^{9}$ also in the case of a circle.

The following corollary to Proposition 3.24 states that line networks with a common factor 2 or 3 for $n$ and $k$ can achieve the optimal value by building optimal strategies following the pattern of Example 3.7 or 3.25 and hence it is presented without proof.

Corollary 3.26. If $g \in G(N)$ is a line and if for $\alpha, \beta \in \mathbb{N}$ : (i) $k=3 \alpha, n=3 \beta$ or (ii) $k=$ $2 \alpha, n=2 \beta$, then there exists $\pi \in \Pi$ such that $V_{k}^{\pi}(g)=V_{k}^{n}$.

\subsection{Adding Links}

Section 3 showed that adding a link to the network while keeping the communication strategy fixed can leave the sender better or worse off. Example 3.2 demonstrates how for a subop- 
timal communication strategy adding a link benefits the sender. Additionally, Example 3.25 illustrates that making a circle from a line can preserve the optimal value.

When the communication strategy is not fixed, however, one might expect that the optimal value (weakly) decreases when a link is added. This initial intuition stems from the fact that the upper bound of the optimal value is reached in the empty network and the lower bound is reached in the complete network. ${ }^{9}$ However, surprisingly, the optimal communication strategy might in fact have a higher value after adding a link, so that the sender benefits from a denser network. This is illustrated by means of an extended example.

Example 3.27. Let $|N|=4, \lambda^{0}(X)=1 / 3$, and $k=2$. Consider the following set of networks starting with $g$, obtained by adding a single link: $g_{1}$ and $g_{2}$ from $g$; the line $g_{3}$ from either $g_{1}$ or $g_{2}$; the star $g_{4}$ from $g_{2}$.

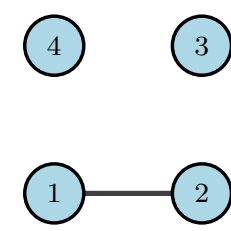

$g: V_{2}^{\pi}(g)=1$

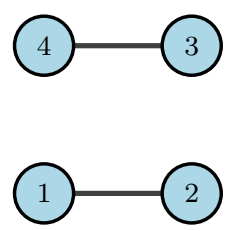

$g_{1}: V_{2}^{\pi}\left(g_{1}\right)=1$

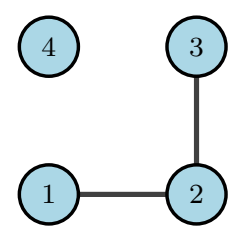

Network $g_{2}$
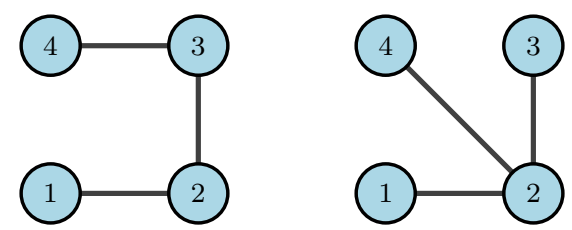

$g_{3}: V_{2}^{\pi^{\prime}}\left(g_{3}\right)=1$ $g_{4}: V_{2}^{\pi^{\prime \prime}}\left(g_{4}\right)=5 / 6$

Consider the maximum values which can be achieved in each case.

\begin{tabular}{c|cc}
$\pi$ & $\omega=X$ & $\omega=Y$ \\
\hline$(x, x, x, x)$ & 1 & 0 \\
$(y, y, x, x)$ & 0 & $\frac{1}{2}$ \\
$(x, x, y, y)$ & 0 & $\frac{1}{2}$
\end{tabular}

\begin{tabular}{c|cc}
$\pi^{\prime}$ & $\omega=X$ & $\omega=Y$ \\
\hline$(x, x, x, x)$ & 1 & 0 \\
$(y, x, x, x)$ & 0 & $\frac{1}{2}$ \\
$(x, x, x, y)$ & 0 & $\frac{1}{2}$
\end{tabular}

Using communication strategy $\pi$ for $g$ and $g_{1}$ and $\pi^{\prime}$ for $g_{3}$, produces support:

$$
\operatorname{supp}\left(\sigma^{g}\right)=\left\{\left(\frac{1}{2}, \frac{1}{2}, \frac{1}{2}, \frac{1}{2}\right),\left(\frac{1}{2}, \frac{1}{2}, 0,0\right),\left(0,0, \frac{1}{2}, \frac{1}{2}\right)\right\} .
$$

and therefore the maximum possible value of 1 in these cases. ${ }^{10}$ In the case of $g_{4}$, it follows from Proposition 3.16 that the optimal value is $V_{2}^{3}=5 / 6$. Notice that slightly adjusting the star network optimal strategy will guarantee a value of $5 / 6$ of $g_{2}$ and so for any $\bar{\pi} \in \Pi$ it holds that $V_{2}^{\bar{\pi}}\left(g_{2}\right) \geq V_{2}^{3}=5 / 6$.

Adding a link between 1 and 4 in $g_{3}$ results in the circle network (call it $g_{5}$ ) analyzed in Example 3.22 with value 1, so starting from $g_{0}$ and adding links in the following way: $g_{0} \rightarrow g \rightarrow g_{1} \rightarrow g_{3} \rightarrow g_{5}$ keeps the optimal value at 1 .

\footnotetext{
${ }^{9}$ Note that this lower bound may also be reached in any other network where the optimal public communication strategy is employed.

${ }^{10}$ Using Proposition 3.11, $\pi$ treats pairs of nodes which observe the same information within a signal (1 and 2, 3 and 4 for $g_{1} ; 1$ and 2 for $g$ ) identically. Additionally, $g_{3}$ is a network which fulfils the conditions of Proposition 3.24 as it is possible to cover precisely $k$ out of the $n$ nodes in every non-anchor signal.
} 
Considering a different sequence like $g \rightarrow g_{2} \rightarrow g_{4}$, adding a link decreases the optimal value. This is not surprising given that the value of the optimal communication strategy with private messages on the empty network dominates (weakly for $n=k$, strongly for $n>k$ ) the value of the public communication strategy (i.e. on the complete network).

What is left to see is if adding a link can strictly increase the maximum value.

Claim 3.28. For any $\hat{\pi} \in \Pi$, it holds that $V_{2}^{\hat{\pi}}\left(g_{2}\right)<1$.

Claim 3.28 implies that adding a link between $g$ and $g_{2}$ strictly decreases the value of the optimal communication strategy, but adding a link between $g_{2}$ and $g_{3}$ strictly increases it. The sequence $g \rightarrow g_{2} \rightarrow g_{3}$ shows that the sender's value may be higher in a denser network.

Given two networks, while the optimal value might be higher in the denser one, the lower bound of optimal values is achieved under the complete network (via the optimal public communication strategy). This provides the highest probability of the true state being implemented among all optimal communication strategies (for any possible network). Thus, even though the receivers in our model are not strategic, since they want the implemented outcome to match the true state, the complete network would provide the highest welfare for the receivers. Hence, if the receivers had the option to disclose their information to their neighbors (without a cost), then they would be willing to create links up to a complete network. In other words, while a denser network is not always beneficial to the receivers, the densest network is the most beneficial.

\subsection{Discussion and Conclusion}

\section{Optimal Strategies}

The implications of Example 3.27 are non-trivial. Overall, constructing a communication strategy for an arbitrary network structure cannot be readily extrapolated from the results which have been outlined and one of the reasons for that is the fact that a less dense network may require a more elaborate strategy to achieve optimal value.

Moreover, even combining identical network components does not provide an easy case. To give an example, consider a star network with $n=4$ and $k=2$. While the optimal value under this network is $V_{2}^{3}=5 / 6$ by Proposition 3.16, combining two such networks in a common network with $n=8$ and $k=4$ allows using Proposition 3.24 and achieving the optimal value $V_{4}^{8}=V_{2}^{4}=1$. However, some trends are observable. Structures with relative symmetry allow for an easier construction of strategies which are optimal (cf. Example 3.23 and 3.25). While no formal proof has been provided for this result, all optimal strategies have anchors which implement $x$. Further, having agents with a very high degree relative to the number of agents in the network (cf. Example 3.15) significantly hinders the persuasion abilities of 
the sender. Hence, from the perspective of the receivers, having many well connected agents in a connected network is a way to limit the sender's influence. This, however, might be challenging if links are costly to maintain.

Additionally, note that while the naive straightforwardness discussed at the end of Section 3.3 does not hold in general (Example 3.7), it is not violated by the optimal strategies in the formally derived propositions.

\section{Equilibrium}

It is natural to ask whether the optimal communication strategies under sincere voting form an equilibrium. As per Kerman et al. (2020), if in a network with $n$ nodes and quota $k<n$ a communication strategy achieves the optimal value as under the empty network, $V_{k}^{n}$, then the strategy does not form a Bayesian Nash equilibrium (BNE) under sincere voting. This is because in optimal strategies agents are never pivotal in state $X$ and are pivotal with positive probability in state $Y$. So, if all other agents vote sincerely, an agent has an incentive to vote against his belief whenever he considers the true state to be $X$ : as he is not pivotal in state $X$, voting against his belief does not change the outcome of the vote if the true state is indeed $X$, while if the true state is $Y$ he is pivotal and should vote against his belief. This exemplifies the swing voter's curse.

One remedy to the swing voter's curse is never making agents pivotal by having at least $k+1$ agents vote for $x$ in state $Y$ (Kerman et al., 2020). A setting where an optimal communication strategy yields a BNE under sincere voting is given in $g_{3}$ in Example 3.21. By Proposition 3.11, if $k=6$ an optimal communication strategy prescribes making all agents vote for $x$ in state $X$ and 8 (not 6 ) out of 12 agents vote for $x$ in state $Y$. Hence, no agent is ever pivotal and sincere voting is a BNE. Similarly, the optimal communication strategy in a complete network is public and yields a BNE under sincere voting.

\section{Concluding Remarks}

This paper investigates the optimal persuasion of voters who exchange private information with each other. This is modeled as a fixed network where direct neighbors can perfectly observe each other's private messages sent by a centralized body. The sender wants to implement a certain proposal and commits in advance to a communication strategy which sends correlated messages to the receivers. This presents several difficulties as most of the assumptions that hold under standard multiple-receiver Bayesian persuasion models fail. Crucially, while there are parallels to the empty network model, straightforward or anonymous strategies are not generally optimal, and neither are strategies which are truth-telling in state $X$.

The paper tests the naive intuition that more information provided to the receivers through the network would make them less manipulable. This is true in some cases. However, the presence of a network structure does not always impede the persuasion abilities of the sender. 
In fact, the value of the optimal communication strategy is not monotone in terms of network density. Several network structures are identified where the sender can achieve the optimal value with private messages as under the empty network and their corresponding optimal strategies are outlined. While many of these situations rely on some form of symmetry or regularity of the network, this can also be achieved on general network structures, e.g. in networks with sufficiently many isolated nodes.

\subsection{Appendix}

Proof of Lemma 3.4. Let $\pi \in \Pi$ be such that $V_{k}^{\pi}(g)>0$. Assume to the contrary that $A n(\pi)=\emptyset$. Then for every $s^{\prime} \in S^{\pi}$ it holds that $\pi\left(s^{\prime} \mid X\right) \lambda^{0}(X)<\pi\left(s^{\prime} \mid Y\right) \lambda^{0}(Y)$. Since $V_{k}^{\pi}(g)>0$, there exists $i \in N$ and $s \in S^{\pi}$ such that $\alpha_{i}^{\pi, g}\left(s_{i}(g)\right)=x$. That is,

$$
\lambda_{i}^{s, g}(X)=\frac{\sum_{t \in A_{i}^{\pi}(g, s)} \pi(t \mid X) \lambda^{0}(X)}{\sum_{t \in A_{i}^{\pi}(g, s)} \pi(t \mid X) \lambda^{0}(X)+\sum_{t \in A_{i}^{\pi}(g, s)} \pi(t \mid Y) \lambda^{0}(Y)} \geq \frac{1}{2} .
$$

Rearranging the terms in (3.4), we obtain:

$$
\sum_{t \in A_{i}^{\pi}(g, s)} \pi(t \mid X) \lambda^{0}(X) \geq \sum_{t \in A_{i}^{\pi}(g, s)} \pi(t \mid Y) \lambda^{0}(Y),
$$

a contradiction, since $\pi\left(s^{\prime} \mid X\right) \lambda^{0}(X)<\pi\left(s^{\prime} \mid Y\right) \lambda^{0}(Y)$ for every $s^{\prime} \in S^{\pi}$.

Notice that this observation implies that every $x$ vote under a strategy with $V_{k}^{\pi}>0$ must be associated with at least one anchor.

Proof of Lemma 3.6. Fix $i \in N$ and let $\left|S_{i}^{\pi}\right| \geq 2$. Note that if $\alpha_{i}^{\pi, g}\left(s_{i}(g)\right)=x$ for some $s \in S^{\pi}$, then $s \in \cup_{t \in A n(\pi)} A^{\pi}(g, t)$, that is, whenever $i$ votes in favor of $x$, his observation is associated with some anchor(s). Moreover, for each $t \in A n(\pi)$ and $s, s^{\prime} \in A_{i}^{\pi}(g, t)$ it holds that $s_{i}=s_{i}^{\prime}=t_{i}$. Note that if $s^{\prime \prime} \notin \cup_{t \in A n(\pi)} A_{i}^{\pi}(g, t)$, then $\alpha_{i}^{\pi, g}\left(s_{i}^{\prime \prime}(g)\right) \neq x$, that is, whenever a receiver observes a private message not associated with any anchor, he does not vote in favor of $x$ and neither do any of his neighbors. That is, for any $j \in N_{i}(g)$ it holds that $\alpha_{j}^{\pi, g}\left(s_{j}^{\prime \prime}(g)\right) \neq x$.

Define $\pi^{\prime}: \Omega \rightarrow \Delta(\Omega)^{n}$ such that in $\pi$ we replace all messages $s_{i} \in S_{i}^{\pi}$ such that $s \notin$ $\cup_{t \in A n(\pi)} A_{i}^{\pi}(g, t)$ with a message $m_{i}^{y} \in S_{i} \backslash S_{i}^{\pi}$. It follows that $A n\left(\pi^{\prime}\right)=A n(\pi)$ and $i$ observes at most $\left|A n\left(\pi^{\prime}\right)\right|+1$ messages under $\pi^{\prime}$, i.e. $\left|S_{i}^{\pi^{\prime}}\right| \leq\left|A n\left(\pi^{\prime}\right)\right|+1$. Let $s \in S^{\pi}$ be such that $s \notin \cup_{t \in A n(\pi)} A_{i}^{\pi}(g, t)$ and therefore, $\alpha_{i}^{\pi, g}\left(s_{i}(g)\right)=y$. If $s^{\prime} \in S^{\pi^{\prime}}$ is obtained from $s \in S^{\pi}$ via replacing $s_{i}$ with $m_{i}^{y}$, then $\alpha_{i}^{\pi^{\prime}, g}\left(s_{i}^{\prime}(g)\right)=x$ if and only if $\alpha_{i}^{\pi, g}\left(s_{i}(g)\right)=x$. That is, the number of $x$ votes are the same under $s$ and $s^{\prime}$. Thus, $V_{k}^{\pi^{\prime}}(g)=V_{k}^{\pi}(g)$.

Proof of Proposition 3.8. Let $\pi \in \Pi$. For each $i \in N$, assume that $\left|S_{i}^{\pi}(g)\right|=c(i)$. Let $R(i)=\left\{m_{i}^{1}, \ldots, m_{i}^{c(i)}\right\} \subseteq S_{i}$ be a set of distinct messages for $i$. Moreover for any $j \in N$, $q \in\{1, \ldots, c(i)\}$, and $q^{\prime} \in\{1, \ldots, c(j)\}$ let $m_{i}^{q} \neq m_{j}^{q^{\prime}}$. 
For each $i \in N$, let $\phi_{i}: S_{i}^{\pi}(g) \rightarrow R(i)$ be a bijection, so each information neighborhood of $i$ is mapped to a unique message in $R(i)$. For each $\omega \in \Omega$ and $s^{\prime} \in S$, define $\pi^{\prime} \in \Pi$ :

$$
\pi^{\prime}\left(s^{\prime} \mid \omega\right)= \begin{cases}\pi(s \mid \omega) & \text { if } \phi_{i}\left(s_{i}(g)\right)=s_{i}^{\prime}, \quad \forall i \in N \\ 0 & \text { otherwise. }\end{cases}
$$

Note that the definition of $\pi^{\prime}$ implies that there is a bijection $\phi: S^{\pi} \rightarrow S^{\pi^{\prime}}$ such that for each $i \in N, \phi(s)=s^{\prime}$ if and only if $\phi_{i}\left(s_{i}(g)\right)=s_{i}^{\prime}$. Hence, $\pi^{\prime}$ is a communication strategy.

We want to show that the value of $\pi^{\prime}$ under the empty network is equal to the value of $\pi$ under $g$, i.e., $V_{k}^{\pi^{\prime}}\left(g_{0}\right)=V_{k}^{\pi}(g)$. What remains to be shown is that each receiver $i$ has the same posterior belief upon observing $s_{i}(g)$ under $\pi$ and upon observing $\phi_{i}\left(s_{i}(g)\right)$ under $\pi^{\prime}$. Let $s^{\prime} \in S^{\pi^{\prime}}$ be such that $s_{i}^{\prime} \in\left\{m_{i}^{1}, \ldots, m_{i}^{c(i)}\right\}$. For any $\omega \in \Omega$, we have

$$
\begin{aligned}
\lambda_{i}^{s^{\prime}}(\omega) & =\frac{\sum_{s \in S^{\pi^{\prime}}: s_{i}=s_{i}^{\prime}} \pi^{\prime}(s \mid \omega) \lambda^{0}(\omega)}{\sum_{\omega^{\prime} \in \Omega} \sum_{s \in S^{\pi^{\prime}}: s_{i}=s_{i}^{\prime}} \pi^{\prime}\left(s \mid \omega^{\prime}\right) \lambda^{0}\left(\omega^{\prime}\right)}=\frac{\sum_{s \in S^{\pi}: s_{i}(g)=\phi^{-1}\left(s_{i}^{\prime}\right)} \pi(s \mid \omega) \lambda^{0}(\omega)}{\sum_{\omega^{\prime} \in \Omega} \sum_{s \in S^{\pi}: s_{i}(g)=\phi^{-1}\left(s_{i}^{\prime}\right)} \pi^{\prime}\left(s \mid \omega^{\prime}\right) \lambda^{0}\left(\omega^{\prime}\right)} \\
& =\frac{\sum_{s \in A_{i}^{\pi}\left(g, \phi^{-1}\left(s^{\prime}\right)\right)} \pi(s \mid \omega) \lambda^{0}(\omega)}{\sum_{\omega^{\prime} \in \Omega} \sum_{s \in A_{i}^{\pi}\left(g, \phi^{-1}\left(s^{\prime}\right)\right)} \pi\left(s \mid \omega^{\prime}\right) \lambda^{0}\left(\omega^{\prime}\right)}=\lambda_{i}^{\phi^{-1}\left(s^{\prime}\right), g}(\omega) .
\end{aligned}
$$

Thus, for each $s \in S^{\pi}$ it holds that $\alpha^{\pi, g}(s)=\alpha^{\pi^{\prime}, g_{0}}(\phi(s))$. Hence, $V_{k}^{\pi^{\prime}}\left(g_{0}\right)=V_{k}^{\pi}(g)$. Since any $\pi \in \Pi$ on some network $g$ can be replicated on the empty network, $V_{k}^{n} \geq V_{k}^{\pi}(g)$.

Proof of Proposition 3.10. Assume that $\lambda^{0}(Y) / \lambda^{0}(X)=\ell,\left|\left\{i \in N: \delta_{i}^{g}=0\right\}\right|=q \geq k$ and $2 k \geq n$. So, there are $q$ singleton receivers and $n-q$ connected receivers. Denote the set of singleton receivers by $N^{q}$ and the set of connected receivers by $N^{c}$.

Let $S^{\prime}=\{x, y\}^{n}$. Define:

$$
R=\left\{s \in S^{\prime}: \forall i \in N^{c}, s_{i}=x \text { and }\left|\left\{j \in N^{q}: s_{j}=x\right\}\right|=k-(n-q)\right\} .
$$

In words, $R$ is the set of signals in which all connected receivers and $k-n+q$ of the singleton receivers observe $x$. Note that $k-(n-q)$ is the required amount of $x$ votes to fulfil the quota given that all connected receivers vote for $x$. Moreover, $|R|=\left(\begin{array}{c}q \\ k-n+q\end{array}\right)$.

Finally, define:

$$
T=\left\{t \in S^{\prime}: \forall i \in N^{c}, t_{i}=y \text { and }\left|\left\{j \in N^{q}: t_{j}=x\right\}\right|=k\right\} .
$$

So, $T$ is the set of signals in which all $n-q$ connected receivers and $q-k$ singleton receivers observe $y$, while $k$ singleton receivers observe $x$. Here $|T|=\left(\begin{array}{l}q \\ k\end{array}\right)$.

Let $\bar{x}$ be such that $\bar{x}_{i}=x$ for all $i \in N$ and define $\bar{y}$ analogously. Define $\pi$ as follows:

$$
\pi(s \mid \omega)= \begin{cases}1 & \text { if } s=\bar{x} \text { and } \omega=X, \\
1-\frac{n}{k \ell} & \text { if } s=\bar{y} \text { and } \omega=Y, \\
\frac{n-k}{q \ell}\left(\begin{array}{c}
q-1 \\
k-1
\end{array}\right)^{-1} & \text { if } s \in T \text { and } \omega=Y, \\
\frac{1}{\left(\begin{array}{c}
q \\
k-n+q
\end{array}\right) \ell} & \text { if } s \in R \text { and } \omega=Y .\end{cases}
$$


It can be easily checked that $\pi$ is a communication strategy:

$$
\begin{aligned}
\sum_{s \in S^{\pi}} \pi(s \mid Y) & =1-\frac{n}{k \ell}+\left(\begin{array}{l}
q \\
k
\end{array}\right) \frac{n-k}{q \ell}\left(\begin{array}{l}
q-1 \\
k-1
\end{array}\right)^{-1}+\left(\begin{array}{c}
q \\
k-n+q
\end{array}\right) \frac{1}{\left(\begin{array}{c}
q \\
k-n+q
\end{array}\right) \ell} \\
& =1-\frac{n}{k \ell}+\frac{n-k}{q \ell} \frac{q}{k}+\frac{1}{\ell}=1-\frac{n}{k \ell}+\frac{n}{k \ell}=1 .
\end{aligned}
$$

We will show that $V_{k}^{\pi}(g)=V_{k}^{n}=\lambda^{0}(X)(n+k) / k$. Under $\pi$, the connected agents always observe the same message. For any $s \in S^{\pi}$ with $s_{i}=x$ for all $i \in N^{c}$, we denote the information neighborhood $s_{i}(g)$ of a connected receiver by $\tilde{x}(i)$. Note that for any $i \in N^{c}$, we have $\pi_{i}(\tilde{x}(i) \mid Y)=\frac{\left(\begin{array}{c}q \\ k-n+q\end{array}\right)}{\left(\begin{array}{c}q \\ k-n+q\end{array}\right) \ell}=1 / \ell$. Hence, for any $i \in N^{c}$ and $s \in S^{\pi}$ with $s_{i}(g)=\tilde{x}(i)$ it holds that:

$$
\lambda_{i}^{s, g}(X)=\frac{\pi_{i}(\tilde{x}(i) \mid X) \lambda^{0}(X)}{\pi_{i}(\tilde{x}(i) \mid X) \lambda^{0}(X)+\pi_{i}(\tilde{x}(i) \mid Y) \lambda^{0}(Y)}=\frac{\lambda^{0}(X)}{\lambda^{0}(X)+\frac{1}{\ell} \lambda^{0}(Y)}=\frac{1}{2} .
$$

Thus, a connected receiver $i$ votes in favor of $x$ upon observing $\tilde{x}(i)$.

Now, let $i \in N^{q}$. The probability of $i$ observing $x$ in state $Y$ is given by:

$$
\begin{aligned}
\pi_{i}(x \mid Y) & =\sum_{s \in S^{\pi}: s_{i}=x} \pi(s \mid Y)=\sum_{s \in R: s_{i}=x} \pi(s \mid Y)+\sum_{t \in T: t_{i}=x} \pi(t \mid Y) \\
& =\frac{\left(\begin{array}{c}
q-1 \\
k-n+q-1
\end{array}\right)}{\left(\begin{array}{c}
q \\
k-n+q
\end{array}\right) \ell}+\left(\begin{array}{l}
q-1 \\
k-1
\end{array}\right) \frac{n-k}{q \ell}\left(\begin{array}{c}
q-1 \\
k-1
\end{array}\right)^{-1}=\frac{k-n+q}{q \ell}+\frac{n-k}{q \ell}=\frac{1}{\ell} .
\end{aligned}
$$

Similar calculations as in the connected receiver case follow and thus, each singleton receiver has posterior $1 / 2$ that the state is $X$ upon observing $x$. The value of $\pi$ is then:

$$
\begin{aligned}
V_{k}^{\pi}(g) & =\lambda^{0}(X) \cdot 1+\lambda^{0}(Y)\left(\frac{n-k}{k \ell}+\frac{1}{\ell}\right)=\lambda^{0}(X)+\lambda^{0}(Y) \frac{n}{k \ell} \\
& =\lambda^{0}(X)+\lambda^{0}(Y) \frac{n}{k} \frac{\lambda^{0}(X)}{\lambda^{0}(Y)}=\frac{n+k}{k} \lambda^{0}(X)=V_{k}^{n} .
\end{aligned}
$$

Proof of Proposition 3.11. First, note that since $\bar{N}_{i}(g)=\bar{N}_{j}(g)$, we have $A_{i}^{\pi}(g, s)=$ $A_{j}^{\pi}(g, s)$. Hence, $i$ and $j$ have the same posterior belief, i.e. for any $\omega \in \Omega$ and any $s \in S^{\pi}$ :

$$
\lambda_{i}^{s, g}(\omega)=\frac{\sum_{t \in A_{i}^{\pi}(g, s)} \pi(t \mid \omega) \lambda^{0}(\omega)}{\sum_{\omega^{\prime} \in \Omega} \sum_{t \in A_{i}^{\pi}(g, s)} \pi\left(t \mid \omega^{\prime}\right) \lambda^{0}\left(\omega^{\prime}\right)}=\frac{\sum_{t \in A_{j}^{\pi}(g, s)} \pi(t \mid \omega) \lambda^{0}(\omega)}{\sum_{\omega^{\prime} \in \Omega} \sum_{t \in A_{j}^{\pi}(g, s)} \pi\left(t \mid \omega^{\prime}\right) \lambda^{0}\left(\omega^{\prime}\right)}=\lambda_{j}^{s, g}(\omega) .
$$

Let $\left|S_{i}^{\pi} \times S_{j}^{\pi}\right|=c$. Let $R=\left\{m^{1}, \ldots, m^{c}\right\}$ be a set of distinct messages. Define a bijection $\phi$ : $S_{i}^{\pi} \times S_{j}^{\pi} \rightarrow R$. That is, for any tuple $\left(s_{i}, s_{j}\right),\left(t_{i}, t_{j}\right) \in S_{i}^{\pi} \times S_{j}^{\pi}$ it holds that $\phi\left(s_{i}, s_{j}\right)=\phi\left(t_{i}, t_{j}\right)$ if and only if $\left(s_{i}, s_{j}\right)=\left(t_{i}, t_{j}\right)$, so that each distinct combination of messages of $i$ and $j$ (and not every distinct neighborhood) is mapped to a distinct message in $R$.

Define $S^{\prime}=\left\{s^{\prime} \in S \mid s \in S^{\pi}, s_{-i j}^{\prime}=s_{-i j}\right.$ and $\left.\phi\left(s_{i}, s_{j}\right)=s_{i}^{\prime}=s_{j}^{\prime} \in R\right\}$. In words, $S^{\prime}$ consists of signals obtained by replacing the messages of $i$ and $j$ with distinct messages in $R$ (for each distinct message combination) and leaving the other receivers' messages unchanged, in each signal in $S^{\pi}$. Let $\tau: S^{\pi} \rightarrow S^{\prime}$ be a bijection such that for any $s \in S^{\pi}$ we have $\tau(s)=s^{\prime}$ if $\tau\left(s_{i}, s_{j}\right)=s_{i}^{\prime}=s_{j}^{\prime}$ and $s_{-i j}^{\prime}=s_{-i j}$. 
For every $s \in S^{\pi}$ and $\omega \in \Omega$, define $\pi^{\prime}(\tau(s) \mid \omega)=\pi(s \mid \omega)$. It is clear that $\pi^{\prime}$ is a communication strategy. Note that since the probability weights are the same under $\pi$ and $\pi^{\prime}$, receivers $i$ and $j$ still have the same posterior belief under $\pi^{\prime}$, i.e. for any $\omega \in \Omega$ and $s \in S^{\pi^{\prime}}$ it holds that $\lambda_{i}^{s, g}(\omega)=\lambda_{j}^{s, g}(\omega)$.

Next, we show that for any $r \in \bar{N}_{i}(g), \omega \in \Omega$, and $s \in S^{\pi}$ we have $\lambda_{r}^{s, g}(\omega)=\lambda_{r}^{\tau(s), g}(\omega)$.

$$
\begin{aligned}
\lambda_{r}^{s, g}(\omega) & =\frac{\sum_{t \in A_{r}^{\pi}(g, s)} \pi(t \mid \omega) \lambda^{0}(\omega)}{\sum_{\omega^{\prime} \in \Omega} \sum_{t \in A_{r}^{\pi}(g, s)} \pi\left(t \mid \omega^{\prime}\right) \lambda^{0}\left(\omega^{\prime}\right)}=\frac{\sum_{t \in A_{r}^{\pi}(g, s)} \pi^{\prime}(\tau(t) \mid \omega) \lambda^{0}(\omega)}{\sum_{\omega^{\prime} \in \Omega} \sum_{t \in A_{r}^{\pi}(g, s)} \pi^{\prime}\left(\tau(t) \mid \omega^{\prime}\right) \lambda^{0}\left(\omega^{\prime}\right)} \\
& =\frac{\sum_{t^{\prime} \in A_{r}^{\pi^{\prime}}(g, \tau(s))} \pi^{\prime}\left(t^{\prime} \mid \omega\right) \lambda^{0}(\omega)}{\sum_{\omega^{\prime} \in \Omega} \sum_{t^{\prime} \in A_{r}^{\pi^{\prime}}(g, \tau(s))} \pi^{\prime}\left(t^{\prime} \mid \omega^{\prime}\right) \lambda^{0}\left(\omega^{\prime}\right)}=\lambda_{r}^{\tau(s), g}(\omega) .
\end{aligned}
$$

Finally, any $r \notin \bar{N}_{i}(g)$ has the same posterior belief under $\pi$ and $\pi^{\prime}$, as it is not affected by the transformation. Hence, $V_{k}^{\pi^{\prime}}(g)=V_{k}^{\pi}(g)$.

Proof of Proposition 3.14. As all components are complete, all of their elements can be sent the same private message within every signal by Proposition 3.11. Let $q \in \mathbb{N}$ be such that for each $\mathcal{C}^{\prime} \in \mathfrak{C}^{q}$ it holds that $\sum_{C \in \mathcal{C}^{\prime}}|C| \geq k$. Note that there are $\left(\begin{array}{l}\ell \\ q\end{array}\right)$ many ways to choose $q$ components such that the total number of receivers in the components is at least $k$. Then, by Theorem 2.8 it follows that there exists $\pi \in \Pi$ such that $V_{k}^{\pi}(g)=V_{q}^{\ell}$.

Proof of Proposition 3.16. First, Lemma 3.29 shows that without loss of generality the center node is not pivotal whenever it votes for $x$. Denote the center node in $g$ by $c \in N$.

Lemma 3.29. Let $g \in G(N)$ be a star, $k<n$, and let $\pi \in \Pi$ be a communication strategy such that there exists $s \in S^{\pi}$ with $\alpha_{c}^{\pi, g}(s)=x$ and $c$ is pivotal in $s$. Then there exists $\pi^{\prime} \in \Pi$ such that for any $s^{\prime} \in S^{\pi^{\prime}}$ with $\alpha_{c}^{\pi^{\prime}, g}\left(s^{\prime}\right)=x$, the center node is not pivotal in $s^{\prime}$ and $V_{k}^{\pi^{\prime}}(g)=V_{k}^{\pi}(g)$.

Proof of Lemma 3.29. Note that $t_{c}(g)=t$ for all $t \in S^{\pi}$ (the information neighbourhood of $c$ is the whole $g$ ). Therefore, $\lambda_{c}^{s, g}(X) \geq 1 / 2$ if and only if $s$ is an anchor, so if $\alpha_{c}^{\pi, g}(s)=x$ for some $s \in S^{\pi}$, it follows that $s \in A n(\pi)$. Moreover, if $c$ is pivotal in this $s$ and $k<n$, there exists a peripheral $i \in N$ (i.e. $i \neq c$ ) such that $\alpha_{i}^{\pi, g}\left(s_{i}(g)\right)=y$. Furthermore, $i$ votes for $y$ in any other signal he associates with $s$, i.e. for any $t \in A_{i}^{\pi}(g, s)$ it holds that $\alpha_{i}^{\pi, g}\left(t_{i}(g)\right)=y$. Thus, replacing $i$ 's message in the anchor $s$ with a unique message would enable $i$ to uniquely identify the anchor and hence reverse $i$ 's vote from $y$ to $x$ in $s$ (see the remark after Definition 3.3). Since $c$ votes for $x$ if and only if the signal is an anchor, $c$ 's vote would not change if the probabilities of the communication strategy do not change. It would also keep everyone else's vote the same, as $i$ is only observed by $c$.

To this end, let $S^{\prime} \subseteq S^{\pi}$ such that $S^{\prime}=\left\{s \in S^{\pi} \mid \alpha_{c}^{\pi, g}(s)=x\right.$ and $c$ is pivotal in $\left.s\right\}$. In particular, let $S^{\prime}=\left\{s^{1}, \ldots, s^{r}\right\}$. Fix $i \in N$ such that for some $t \in S^{\prime}$ it holds that $\alpha_{i}^{\pi, g}(t)=y$ and notice that such $i$ exists as per the discussion above. Let $R=\left\{m^{1}, \ldots, m^{r}\right\}$ be a set of distinct messages such that for any $j \in\{1, \ldots, r\}, m^{j} \notin S_{i}^{\pi}$. Let $S^{\prime \prime} \subseteq S$ and define a bijection 
$\phi: S^{\prime} \rightarrow S^{\prime \prime}$ such that for every $j \in\{1, \ldots, r\}$ and $s^{j} \in S^{\prime}$ it holds that $\phi_{i}\left(s_{i}^{j}\right)=m^{j}$ and $\phi_{-i}\left(s_{-i}^{j}\right)=s_{-i}^{j}$.

Now, for any $\omega \in \Omega$ and any $s^{\prime} \in\left(S^{\pi} \backslash S^{\prime}\right) \cup S^{\prime \prime}$, let $\pi^{\prime} \in \Pi$ be defined by:

$$
\pi^{\prime}\left(s^{\prime} \mid \omega\right)= \begin{cases}\pi\left(\phi^{-1}\left(s^{\prime}\right) \mid \omega\right) & \text { if } s^{\prime} \in S^{\prime \prime}, \\ \pi\left(s^{\prime} \mid \omega\right) & \text { if } s^{\prime} \in S^{\pi} \backslash S^{\prime} .\end{cases}
$$

That is, $S^{\pi^{\prime}}=\left(S^{\pi} \backslash S^{\prime}\right) \cup S^{\prime \prime}$. By the definition of an anchor, for any $t \in S^{\prime \prime}$, we have $\alpha_{i}^{\pi^{\prime}, g}\left(t_{i}(g)\right)=x$, since by construction $i$ observes the unique message only in this anchor $t$. Therefore, there are $k+1$ receivers voting for $x$ in $t$, which implies that the center node is no longer pivotal. Since $\pi^{\prime}$ preserves all probability weights it is true that if $s \in S^{\prime \prime}$, then $s \in A n\left(\pi^{\prime}\right)$.

Moreover, $i$ 's votes in signals that are not in $S^{\prime \prime}$ are unchanged, i.e. for any $t \in S^{\pi^{\prime}} \backslash S^{\prime \prime}$, $\alpha_{i}^{\pi, g}\left(t_{i}(g)\right)=\alpha_{i}^{\pi^{\prime}, g}\left(t_{i}(g)\right)$. This holds because if $s \in S^{\prime}$ and $t \in A_{i}^{\pi}(g, s)$, then it holds that $\alpha_{i}^{\pi, g}\left(t_{i}(g)\right)=y$ by the definition of $S^{\prime}$ and the selection of $i$. The transformation removes the anchors in $S^{\prime \prime}$ from the association set of every signal $t \in S^{\pi^{\prime}} \backslash S^{\prime \prime}$, so if $s \in S^{\prime \prime}$ then for every $t \in S^{\pi^{\prime}} \backslash S^{\prime \prime}$ it is true that $t \notin A_{i}^{\pi^{\prime}}(g, s)$. This makes it even less likely that $i$ would vote for $x$ in such signals, preserving its $y$ votes in them between $\pi$ and $\pi^{\prime}$. The transformation does not affect any other receivers' votes, hence $V_{k}^{\pi^{\prime}}(g)=V_{k}^{\pi}(g)$.

By Lemma 3.29, assume without loss of generality that under $\pi$, the center node is not pivotal in signals in which he votes for $x$.

For all nodes $i \in N$ and all $t \notin A n(\pi)$, if $t_{c} \neq s_{c}$ for all $s \in A n(\pi)$ then $\lambda_{i}^{t, g}(X)<1 / 2$. So, if in a certain signal the center of a star network receives a message different from all anchors, all receivers would vote $y$ in this signal.

Note that for two anchors $s, t \in A n(\pi)$ with $s_{c} \neq t_{c}$, it holds that $A^{\pi}(g, s) \cap A^{\pi}(g, t)=\emptyset$. Define a bijection $\phi: S^{\pi} \rightarrow S^{\prime}$ such that $\phi(s)=s^{\prime}$ if $s_{c}^{\prime}=x$ and for every $j \in N \backslash\{c\}$, $s_{j}^{\prime}=\left(s_{j}, s_{c}\right)$. That is, in signals in $S^{\prime}$ the center node always observes $x$ and the periphery nodes' messages are modified so that they contain the information previously provided by the center in signal $s$. In other words, the information that the center reveals to the periphery nodes is shifted to them while the center observes the same message $x$ in every signal.

For every $s^{\prime} \in S^{\prime}$ such that $\phi(s)=s^{\prime}$ and $\omega \in \Omega$, let $\pi^{\prime} \in \Pi$ be defined by $\pi^{\prime}\left(s^{\prime} \mid \omega\right)=$ $\pi\left(\phi^{-1}\left(s^{\prime}\right) \mid \omega\right)$. As the probabilities of corresponding signals are the same under $\pi^{\prime}$ as under $\pi$ and the center's information under $\pi$ is shifted to the periphery nodes under $\pi^{\prime}$ (which are observed by the center), the center node's vote does not change. Moreover, the votes of the periphery nodes do not change either. To see this, note that for any $t^{\prime} \in A_{i}^{\pi^{\prime}}\left(g, s^{\prime}\right)$ there exists $t \in A_{i}^{\pi}(g, s)$ such that $\phi(t)=t^{\prime}$. This, together with the definition of $\phi$ implies that $\sum_{t^{\prime} \in A_{i}^{\pi^{\prime}\left(g, s^{\prime}\right)}} \pi^{\prime}\left(t^{\prime} \mid \omega\right)=\sum_{t \in A_{i}^{\pi}(g, s)} \pi(t \mid \omega)$. Thus, each periphery node has the same posterior belief upon observing $s \in S^{\pi}$ and $\phi(s) \in S^{\pi^{\prime}}$. Hence, $V_{k}^{\pi^{\prime}}(g)=V_{k}^{\pi}(g)$. 
As the center node always observes the same message under $\pi^{\prime}$, it has no effect on the voting decisions of the other receivers. Moreover, the center node is never pivotal in signals in which he votes for $x$. Observe that under $\pi^{\prime}$, it is as if the center is always voting for $y$, since all of his $y$ votes are preserved in $\pi^{\prime}$ and none of his $x$ votes have an impact on whether a signal implements $x$ or not. Thus, the setup is equivalent to having an empty network with $n-1$ nodes. Hence, we can assume without loss of generality that there exists a communication strategy $\pi^{\prime \prime} \in \Pi$ with $\left|S_{i}^{\pi^{\prime \prime}}\right|=2$ for any $i \in N$ such that $V_{k}^{n-1}=V_{k}^{\pi^{\prime \prime}}(g) \geq V_{k}^{\pi^{\prime}}(g)$.

Proof of Proposition 3.18. Take the following communication strategy $\pi$ :

\begin{tabular}{|c|c|c|}
\hline$\pi$ & $\omega=X$ & $\omega=Y$ \\
\hline$(x, x, x, x, \ldots, x, x, x, x)$ & 1 & 0 \\
\hline$(\underbrace{y, \ldots, y}, x, x, x, \ldots, x, x)$ & 0 & $\frac{w_{1}}{n}$ \\
\hline$(x, \underbrace{y, \ldots, y}_{a}, x, x, \ldots, x, x)$ & 0 & $\frac{w_{1}}{n}$ \\
\hline$(x, x, \ldots, x, x, \underbrace{y, \ldots, y}_{a}, x)$ & 0 & $\frac{w_{1}}{n}$ \\
\hline$(x, x, \ldots, x, x, x, \underbrace{y, \ldots, y}_{a})$ & 0 & $\frac{w_{1}}{n}$ \\
\hline$(\underbrace{y, \ldots, y}_{a-1}, x, x, \ldots, x, x, y)$ & 0 & $\frac{w_{1}}{n}$ \\
\hline$(y, y, y, y, \ldots, y, y, y, y)$ & 0 & $w_{2}$ \\
\hline
\end{tabular}

with $w_{1}=r_{k}^{*}, w_{2}=r_{0}^{*}$ from Theorem 2.8 and $a=n-2-k$. This makes a total of $n+2$ signals. Every node observes a message $y$ in their information neighborhood in exactly $a+3$ signals. This leaves $n-1-a$ signals in which $i$ and all neighbors of $i$ observe $x$. Given $s^{\prime} \in S^{\pi}$, denote the information neighborhood $s_{i}^{\prime}(g)$ of $i \in N$ by $\tilde{x}(i)$ if for all $j \in \bar{N}_{i}(g)$ it holds that $s_{j}^{\prime}=x$. Let $i \in N$ and $s \in S^{\pi}$ be such that $s_{i}(g)=\tilde{x}(i)$. It holds that:

$$
\lambda_{i}^{s, g}(X)=\frac{\sum_{t \in A_{i}^{\pi}(g, s)} \pi(t \mid X) \lambda^{0}(X)}{\sum_{t \in A_{i}^{\pi}(g, s)} \pi(t \mid X) \lambda^{0}(X)+\sum_{t \in A_{i}^{\pi}(g, s)} \pi(t \mid Y) \lambda^{0}(Y)}=\frac{\lambda^{0}(X)}{\lambda^{0}(X)+\frac{(n-2-a) w_{1}}{n} \lambda^{0}(Y)} .
$$

Therefore,

$$
\lambda_{i}^{s, g}(X)= \begin{cases}\frac{\lambda^{0}(X)}{1 \lambda^{0}(X)+\frac{(n-2-a)}{n} \frac{\lambda^{0}(X) n}{\lambda^{0}(Y) k} \lambda^{0}(Y)}=\frac{1}{1+\frac{n-2-a}{k}}=1 / 2 & \text { if } \lambda^{0}(X)<\frac{k}{k+n}, \\ \frac{\lambda^{0}(X)}{\lambda^{0}(X)+(n-2-a) \lambda^{0}(Y) / n} \geq 1 / 2 & \text { if } \lambda^{0}(X) \geq \frac{k}{k+n},\end{cases}
$$

as the second condition always holds for $\lambda^{0}(X) \geq \frac{k}{k+n}$. 
In each signal $s \in S^{\pi}$ such that there exists $i \in N$ with $s_{i}=y$, there are $n-2-a$ many receivers $j \in N$ such that $s_{j}(g)=\tilde{x}(j)$. Therefore, all these signals persuade at least $k$ agents, i.e. for such $s \in S^{\pi}$ we have $\left|\left\{i \in N: \alpha_{i}^{\pi, g}\left(s_{i}(g)\right)=x\right\}\right| \geq k$. The value is equal to the empty network value, i.e. $V_{k}^{\pi}(g)=\lambda^{0}(X) \cdot 1+\lambda^{0}(Y) w_{1}=\min \left\{\frac{n+k}{k}, 1\right\}=V_{k}^{n}$.

Proof of Proposition 3.20. Let $S^{\prime}=\{x, y\}^{n}$. Let $T$ be the set of signals in $S^{\prime}$ in which exactly one receiver observes $y$, i.e. $T=\left\{s \in S^{\prime} \mid \exists i \in N: s_{i}=y\right.$ and $\left.s_{-i}=x\right\}$. Let $\bar{x} \in S$ be such that $\bar{x}_{i}=x$ for all $i \in N$ and define $\bar{y}$ analogously. Define $\pi \in \Pi$ :

$$
\pi(s \mid \omega)= \begin{cases}1 & \text { if } s=\bar{x} \text { and } \omega=X \\ \frac{w_{1}}{n} & \text { if } s \in T \text { and } \omega=Y \\ w_{2} & \text { if } s=\bar{y} \text { and } \omega=Y \\ 0 & \text { otherwise }\end{cases}
$$

with $w_{1}=r_{k}^{*}, w_{2}=r_{0}^{*}$ from Theorem 2.8. This makes a total of $n+2$ signals. Since the network is regular, every node observes message $y$ in their information neighborhood in exactly $\delta+2$ signals. This leaves $n-\delta$ signals where an agent $i \in N$ observes $\tilde{x}(i)$ (in particular, there are $n-\delta-1$ such signals in state $Y)$. Let $i \in N$ and $s \in S^{\pi}$ be such that $s_{i}(g)=\tilde{x}(i)$. It holds that:

$$
\lambda_{i}^{s, g}(X)=\frac{\sum_{t \in A_{i}^{\pi}(g, s)} \pi(t \mid X) \lambda^{0}(X)}{\sum_{t \in A_{i}^{\pi}(g, s)} \pi(t \mid X) \lambda^{0}(X)+\sum_{t \in A_{i}^{\pi}(g, s)} \pi(t \mid Y) \lambda^{0}(Y)}=\frac{\lambda^{0}(X)}{\lambda^{0}(X)+\frac{(n-\delta-1) w_{1}}{n} \lambda^{0}(Y)} .
$$

Therefore,

$$
\lambda_{i}^{s, g}(X)= \begin{cases}\frac{1 \lambda^{0}(X)}{1 \lambda^{0}(X)+\frac{(n-\delta-1)}{n} \frac{\lambda^{0}(X) n}{\lambda^{0}(Y) k} \lambda^{0}(Y)}=\frac{1}{1+\frac{n-\delta-1}{k}}=\frac{1}{2} & \text { if } \lambda^{0}(X)<\frac{k}{k+n} \\ \frac{\lambda^{0}(X)}{\lambda^{0}(X)+\frac{k}{n} \lambda^{0}(Y)} \geq 1 / 2 & \text { if } \lambda^{0}(X) \geq \frac{k}{k+n}\end{cases}
$$

as the second condition always holds for for $\lambda^{0}(X) \geq \frac{k}{k+n}$.

In each signal $s \in S^{\pi}$ such that there exists $i \in N$ with $s_{i}=y$, there are $n-1-\delta=k$ many receivers $j \in N$ such that $s_{j}(g)=\tilde{x}(j)$. Therefore all these signals persuade exactly $k$ agents, i.e., for such $s \in S^{\pi}$ we have $\left|\left\{i \in N: \alpha_{i}^{\pi, g}\left(s_{i}(g)\right)=x\right\}\right| \geq k$ The value is equal to the empty network value, $V_{k}^{\pi}(g)=\lambda^{0}(X) \cdot 1+\lambda^{0}(Y) w_{1}=\min \left\{\frac{n+k}{k}, 1\right\}=V_{k}^{n}$.

Proof of Proposition 3.24. In the empty network the value corresponding to $k=q \alpha, n=$ $q \beta$ is the same as the value for $k^{\prime}=\alpha, n^{\prime}=\beta$, since $V_{k}^{n}\left(\lambda^{0}\right)=\min \left\{\frac{n+k}{k} \lambda^{0}(X), 1\right\}$ (Theorem 2.8) and $\frac{n+k}{k} \lambda^{0}(X)=\frac{q \alpha+q \beta}{q \beta} \lambda^{0}(X)=\frac{\alpha+\beta}{\beta} \lambda^{0}(X)$. Therefore, if the network allows uniform treatment of parts with the minimal necessary size $(q)$ so that an equal number of nodes in every part has a neighborhood with at least one $y$ message in it, the setup becomes equivalent to the empty network and allows obtaining the optimal value with private communication, so that if $V_{k}^{n}=V_{q \alpha}^{q \beta}$, then $V_{k}^{n}=V_{\alpha}^{\beta}$. 
Proof of Claim 3.28. Assume the opposite, i.e. $V_{2}^{\hat{\pi}}\left(g_{2}\right)=1$. Therefore:

- $\hat{\pi}$ is optimal on $g_{2}$ and for every $t \in S^{\hat{\pi}}, t \in Z_{x}^{g_{2}}(\hat{\pi})$, so all signals in $\hat{\pi}$ implement $x$.

- For every $t \in Z_{x}^{g_{2}}(\hat{\pi})$, there exists $s \in A n(\hat{\pi})$ such that $s_{2}=t_{2}$. If $t_{2} \neq s_{2}^{\prime}$ for all $s^{\prime} \in A n(\hat{\pi})$, then agents 1,2 and 3 would vote for $y$ and the signal would not implement $x$.

- Node 4 never receives any information from any other node, so changing anything in the other nodes' messages would not change the vote of 4 .

Observe that if player 2 is never pivotal, then any transformation of the communication strategy which preserves the other nodes' votes does not change the value of the strategy.

Claim 3.30. For any $\hat{\pi} \in \Pi$ with $V_{2}^{\hat{\pi}}\left(g_{2}\right)=1$ there exists $\hat{\pi}^{\prime} \in \Pi$ such that: (i) 2 is never pivotal, (ii) $\left|S_{2}^{\hat{\pi}^{\prime}}\right|=1$ and (iii) $V_{2}^{\hat{\pi}^{\prime}}\left(g_{2}\right)=V_{2}^{\hat{\pi}}\left(g_{2}\right)$.

If the claim holds, then node 2 never reveals or receives any consequential information (in terms of value). That is, the situation would be equivalent to an empty network with $n=3$ and $k=2$ where $V_{2}^{\hat{\pi}^{\prime}}\left(g_{2}\right) \leq V_{2}^{3}=5 / 6<1$, which would contradict the initial assumption.

Proof of Claim 3.30. (i) It is clear that for $s \in S^{\hat{\pi}}$ with $\alpha_{2}^{\hat{\pi}, g_{2}}\left(s_{2}\left(g_{2}\right)\right)=y$, node 2 is not pivotal since all signals implement $x$.

Suppose that there is a signal $t \in S^{\hat{\pi}}$ in which node 2 votes for $x$. Hence, there is at least one anchor $s \in A n(\hat{\pi})$ with $\left(s_{1}, s_{2}, s_{3}\right)=\left(t_{1}, t_{2}, t_{3}\right)$ and for every $r \in S^{\hat{\pi}}$ such that $\left(r_{1}, r_{2}, r_{3}\right)=\left(s_{1}, s_{2}, s_{3}\right)$, node 2 also votes for $x$. The possible voting patterns of nodes 1,2 and 3 in such signals are: $(a)(x, x, x) ;(b)(y, x, x) ;(c)(x, x, y) ;(d)(y, x, y)$.

In the case $(a), 2$ is not pivotal. Consider case $(b)$. It must be true that node 1 votes for $y$ because it associates $t$ with more signals than 2. In other words, in all signals $r \in S^{\hat{\pi}}$ where $\left(r_{1}, r_{2}\right)=\left(t_{1}, t_{2}\right)$ node 1 votes for $y$ and this includes the signals in which 2 does not vote for $x$. (This also includes the associated anchors.) Thus, $A_{2}^{\pi}\left(g_{2}, t\right) \subsetneq A_{1}^{\pi}\left(g_{2}, t\right)$.

Notice the trivial fact that for every $s, t \in S^{\hat{\pi}}$ with $s_{2} \neq t_{2}$ and $i \in\{1,2,3\}$, it holds that $A_{i}^{\pi}\left(g_{2}, s\right) \cap A_{i}^{\pi}\left(g_{2}, t\right)=\emptyset$, so that whenever node 2 receives a different message in different signals, these signals belong to disjoint association sets (for node 2) and the same observation holds for its neighbors, 1 and 2 .

Let $S_{2}^{\hat{\pi}}=\left\{m^{1}, \ldots, m^{\ell}\right\}$ and define the set of signals in which receiver 1 votes for $y$ and receiver 2 votes for $x$ as $T=\left\{t \in S^{\hat{\pi}} \mid \alpha_{1}^{\hat{\pi}, g_{2}}\left(t_{1}\left(g_{2}\right)\right)=y\right.$ and $\left.\alpha_{2}^{\hat{\pi}, g_{2}}\left(t_{2}\left(g_{2}\right)\right)=x\right\}$.

Define a bijection such that in signals in $\hat{\pi}$ in which 1 votes for $y$ and 2 votes for $x$, we change the message of 1 to a unique message that is specific to each distinct message of 2 and keep all other messages the same. Formally, let $T^{\prime} \subsetneq S$ and define $\phi: T \rightarrow T^{\prime}$ such that for any $t \in T$ it holds that $\phi(t)=t^{\prime}$ if $t_{1}^{\prime}=\left(t_{1}, t_{2}\right) \in S_{1}^{\prime} \backslash S_{1}^{\hat{\pi}}$ and $t_{-1}^{\prime}=t_{-1}$. 
Now for any $\omega \in \Omega$ define a new strategy $\hat{\pi}^{\prime} \in \Pi$, which transforms the signals in $T$ according to $\phi$, keeps all other signals the same while preserving the probability weights:

$$
\hat{\pi}^{\prime}\left(s^{\prime} \mid \omega\right)= \begin{cases}\hat{\pi}\left(s^{\prime} \mid \omega\right) & \text { if } s^{\prime} \in S^{\hat{\pi}} \backslash T, \\ \hat{\pi}\left(\phi^{-1}\left(s^{\prime}\right) \mid \omega\right) & \text { if } s^{\prime} \in T .\end{cases}
$$

Let $s^{\prime} \in S^{\hat{\pi}^{\prime}}$ be such that $\phi(s)=s^{\prime}$ for some $s \in T$. Then,

$$
\begin{aligned}
& \lambda_{1}^{s^{\prime}, g_{2}}(X)=\frac{\sum_{t^{\prime} \in A_{1}^{\hat{\pi}^{\prime}}\left(g_{2}, s^{\prime}\right)} \hat{\pi}^{\prime}\left(t^{\prime} \mid X\right) \lambda^{0}(X)}{\sum_{\omega \in \Omega} \sum_{t^{\prime} \in A_{1}^{\hat{\pi}^{\prime}}\left(g_{2}, s^{\prime}\right)} \hat{\pi}^{\prime}\left(t^{\prime} \mid \omega\right) \lambda^{0}(\omega)}=\frac{\sum_{t^{\prime} \in A_{1}^{\hat{\pi}^{\prime}}\left(g_{2}, s^{\prime}\right)} \hat{\pi}\left(\phi^{-1}\left(t^{\prime}\right) \mid X\right) \lambda^{0}(X)}{\sum_{\omega \in \Omega} \sum_{t^{\prime} \in A_{1}^{\hat{\pi}^{\prime}}\left(g_{2}, s^{\prime}\right)} \hat{\pi}\left(\phi^{-1}\left(t^{\prime}\right) \mid \omega\right) \lambda^{0}(\omega)} \\
& =\frac{\sum_{t \in A_{1}^{\hat{\pi}}\left(g_{2}, s\right) \cap A_{2}^{\hat{\pi}}\left(g_{2}, s\right)} \hat{\pi}(t \mid X) \lambda^{0}(X)}{\sum_{\omega \in \Omega} \sum_{t \in A_{1}^{\hat{\pi}}\left(g_{2}, s\right) \cap A_{2}^{\hat{\pi}}\left(g_{2}, s\right)} \hat{\pi}(t \mid \omega) \lambda^{0}(\omega)}=\frac{\sum_{t \in A_{2}^{\hat{\pi}}\left(g_{2}, s\right) \subseteq T} \hat{\pi}(t \mid X) \lambda^{0}(X)}{\sum_{\omega \in \Omega} \sum_{t \in A_{2}^{\hat{\pi}}\left(g_{2}, s\right) \subseteq T} \hat{\pi}(t \mid \omega) \lambda^{0}(\omega)} \geq \frac{1}{2},
\end{aligned}
$$

where $\phi(t)=t^{\prime}$ and the third equality follows from the definition of $\phi ; A_{1}^{\hat{\pi}}\left(g_{2}, s\right) \cap A_{2}^{\hat{\pi}}\left(g_{2}, s\right)=$ $A_{2}^{\hat{\pi}}\left(g_{2}, s\right) \subseteq T$ follows from $A_{2}^{\pi}\left(g_{2}, t\right) \subsetneq A_{1}^{\pi}\left(g_{2}, t\right)$ and the inequality follows from the definition of case $(b)$. Similarly, it holds that $\lambda_{2}^{s^{\prime}, g_{2}}(X) \geq 1 / 2$. This implies that in $\hat{\pi}^{\prime}$ node 1 will vote for $x$ whenever 2 votes for $x$ in $\hat{\pi}^{\prime}$. Additionally, node 2 will keep its vote for $x$ in the corresponding signals in $\hat{\pi}$ and $\hat{\pi}^{\prime}$. Thus, the transformation does not change the vote of 2 in any signals. It only increases the number of $x$ votes in signals which already implement $x$ (since all signals must do under $\hat{\pi}$ ). Observe that for $s \in A_{1}^{\pi}\left(g_{2}, t\right) \backslash A_{2}^{\pi}\left(g_{2}, t\right)$ such that $t \in T$, it holds that $\alpha_{1}^{\hat{\pi}, g_{2}}\left(t_{1}\left(g_{2}\right)\right)=y$ and the transformation will not decrease the value, as in such $s$ nodes 1 and 2 must already be voting for $y$. Hence, $V_{2}^{\hat{\pi}^{\prime}}\left(g_{2}\right)=V_{2}^{\hat{\pi}}\left(g_{2}\right)$. A similar transformation can be applied in cases $(c)$ and $(d)$.

Therefore, for any communication strategy $\hat{\pi}$ with $V_{k}^{\hat{\pi}}\left(g_{2}\right)=1$, there exists $\hat{\pi}^{\prime} \in \Pi$ such that in every signal in which 2 votes for $x$ in $\hat{\pi}$, nodes 1,2 and 3 vote for $x$ in $\hat{\pi}^{\prime}$ such that $V_{2}^{\hat{\pi}^{\prime}}\left(g_{2}\right)=V_{2}^{\hat{\pi}}\left(g_{2}\right)$. Thus, 2 is never pivotal in $\hat{\pi}^{\prime}$.

(ii) Keeping the message of node 4 the same as in $\hat{\pi}$ (and in $\hat{\pi}^{\prime}$ ), from here onwards, the transformation is the same as in the star network (see proof of Proposition 3.16). Note that for two anchors $s, t \in S^{\hat{\pi}}$ with $s_{2} \neq t_{2}$, it holds that $A_{2}^{\pi}\left(g_{2}, s\right) \cap A_{2}^{\pi}\left(g_{2}, t\right)=\emptyset$. Let $S^{\prime} \subseteq S$. Define a bijection $\tau: S^{\hat{\pi}^{\prime}} \rightarrow S^{\prime}$ such that $\tau(s)=s^{\prime}$ if $s_{2}^{\prime}=x$, for $j \in\{1,3\}, s_{j}^{\prime}=\left(s_{j}, s_{2}\right)$, and $s_{4}^{\prime}=s_{4}$. That is, in signals in $S^{\prime}$ node 2 always observes $x$ and the messages of node 1 and 3 are modified so that they contain the information previously provided by node 2 in signal $s$. In other words, the information that node 2 reveals to node 1 and 3 is shifted to them while node 2 observes the same message in every signal.

For any $s^{\prime} \in S^{\prime}$ such that $\tau(s)=s^{\prime}$ and $\omega \in \Omega$, let $\hat{\pi}^{\prime \prime} \in \Pi$ be defined by $\hat{\pi}^{\prime \prime}\left(s^{\prime} \mid \omega\right)=$ $\hat{\pi}^{\prime}\left(\tau^{-1}\left(s^{\prime}\right) \mid \omega\right)$. As the probabilities of corresponding signals are the same under $\hat{\pi}^{\prime \prime}$ as under $\hat{\pi}^{\prime}$ and node 2's information under $\hat{\pi}^{\prime}$ is shifted to nodes 1 and 3 under $\hat{\pi}^{\prime \prime}$ (which are observed by node 2), node 2's vote does not change. Moreover, the votes of nodes 1,3 , and 4 do not change either. To see this, note that for any $i \in\{1,3,4\}$ and $t^{\prime} \in A_{i}^{\hat{\pi}^{\prime \prime}}\left(g, s^{\prime}\right)$ there 
exists $t \in A_{i}^{\hat{\pi}^{\prime}}(g, s)$ such that $\tau(t)=t^{\prime}$. This, together with the definition of $\tau$ implies that $\sum_{t^{\prime} \in A_{i}^{\hat{\pi}^{\prime \prime}}\left(g, s^{\prime}\right)} \hat{\pi}^{\prime \prime}\left(t^{\prime} \mid \omega\right)=\sum_{t \in A_{i}^{\hat{\pi}^{\prime}(g, s)}} \hat{\pi}^{\prime}(t \mid \omega)$. Thus, every node has the same posterior belief upon observing $s \in S^{\hat{\pi}^{\prime}}$ and $\tau(s) \in S^{\hat{\pi}^{\prime \prime}}$.

(iii) Parts $(i)$ and $(i i)$ imply that the value is preserved, i.e. $V_{2}^{\hat{\pi}^{\prime \prime}}\left(g_{2}\right)=V_{2}^{\hat{\pi}^{\prime}}\left(g_{2}\right)=V_{2}^{\hat{\pi}}\left(g_{2}\right)$.

Hence, for $k=2$ a communication strategy $\hat{\pi}$ with $V_{2}^{\hat{\pi}}\left(g_{2}\right)=1$ can be transformed into a strategy such that: node 2 is never pivotal, it always receives the same message and the strategy preserves the value of the initial strategy. This proves Claim 3.30.

As Claim 3.30 holds, we have $V_{2}^{\hat{\pi}}\left(g_{2}\right) \leq 5 / 6$, which contradicts the initial assumption that $V_{2}^{\hat{\pi}}\left(g_{2}\right)=1$. Therefore, $V_{2}^{\hat{\pi}}\left(g_{2}\right)<1$, which proves Claim 3.28. 


\section{Chapter 4}

\section{Belief Inducibility and Informativeness}

"Belief can be manipulated. Only knowledge is dangerous."

- Frank Herbert

This chapter is adapted from Herings, Karos, and Kerman (2020). 
In this chapter, we consider a group of receivers who share a common prior on a finite state space and who observe private correlated signals that are contingent on the true state of the world. We show that, while necessary, Bayes plausibility is not sufficient for a distribution over posterior belief vectors to be inducible, and we provide a characterization of inducible distributions. We classify communication strategies as minimal, direct, and language independent, and we show that any inducible distribution can be induced by a language independent communication strategy (LICS). We investigate the role of the different classes of communication strategies for the amount of higher order information that is revealed to receivers. We show that the least informative communication strategy which induces a fixed distribution over posterior belief vectors lies in the relative interior of the set of all language independent communication strategies which induce that distribution.

\subsection{Introduction}

In any economic model which involves a group of agents and has a payoff structure that depends on the posterior beliefs of the agents, one of the essential questions is "Which distributions over posterior beliefs of agents can be induced?" In their seminal paper, Kamenica and Gentzkow (2011) consider communication between a sender and a receiver who share a common prior and show that the only restriction on the set of inducible distributions over posterior belief vectors is Bayes plausibility: the expected posterior belief is equal to the prior. ${ }^{1}$ It follows from their insight that Bayes plausibility and identical beliefs is necessary and sufficient in the case of multiple receivers and public communication, that is, when messages are perfectly correlated. Yet, in this case the set of inducible distributions over posteriors is very limited since all receivers have the same ex-post belief. In the present paper we are interested in private communication, which, in contrast, enables the sender to achieve a richer belief space; however, Bayes plausibility is no longer sufficient for inducibility. In other words, there are distributions which are Bayes plausible for every agent, but are not induced by any communication strategy. The first question we answer is: what additional conditions are required for the existence of such a communication strategy?

Another aspect which is important for both the sender and receivers is the informativeness of a communication strategy. In the original information design setup introduced by Aumann, Machler, and Stearns (1995), the authors were interested in communication that reveals as little private information as possible. In our paper, a signal realization does not only reveal information about the true state of the world: as there are multiple receivers who each obtain a private message, it also induces information partitions that determine what any receiver knows about another receiver's knowledge of the true state and the signal realization. Thus,

\footnotetext{
${ }^{1}$ This is also known as the martingale property.
} 
we compare the informativeness of communication strategies in terms of "knowledge" in the sense of Hintikka (1962). Finer information partitions correspond to more informative communication strategies. The second main question we answer is: what types of communication strategies are the least informative? That is, we first find which distributions of posterior beliefs are feasible for the sender, and then provide a characterization for least informative communication strategies that induce a posterior distribution. Our approach towards informativeness is different from the one of Blackwell (1953) and does not depend on conditional probability distributions over states for instance.

We consider a sender who commits to a communication strategy that sends private correlated messages to the receivers. Receivers know the joint the distribution of signals, that is vectors of messages, but they only observe their own private message from the signal realization. We first show that there are posterior belief vectors, which the sender cannot achieve with positive probability. More precisely, for a given posterior belief vector, there exists a communication strategy that induces a distribution which puts positive weight on it if and only if there exists a state which is deemed possible by all receivers according to this belief vector. As an example, consider an operative who follows Machiavelli's advice divide et impera and, thus, wants to create political unrest in a foreign country by implementing a very heterogeneous belief vector. Suppose that there are only two states, say blue and red. Then it is impossible for the operative to implement a distribution that puts positive weight on a posterior belief vector in which one receiver believes the state is blue with probability 1 and another receiver beliefs that the state is red with probability 1. At the same time, a posterior belief vector in which the first receiver's belief that the state is blue is equal to 1 , and the second receiver's belief is arbitrarily close to 0 can be achieved with positive probability.

We next define particular classes of communication strategies. We first consider minimal communication strategies under which distinct signals lead to distinct posterior belief vectors. While this ensures that no two signals implement the same posterior belief vector, there might still be individual receivers for whom different messages lead to the same posterior. If for each receiver every posterior is induced by a unique message, the communication strategy is called direct. If, additionally, the sent messages are themselves posteriors such that each message induces itself, we call the communication strategies language independent (LICS). Here, a sender simply tells the receivers what belief they should have, and the messages are sent with probabilities such that receivers will believe the message. We show that restricting attention to language independent communication strategies is without loss of generality, that is, if a posterior distribution can be induced, it can be induced by an LICS.

As mentioned before, in the presence of multiple receivers Bayes plausibility is necessary but not sufficient for a distribution to be inducible. We characterize the set of inducible distributions of posteriors by showing that a Bayes plausible distribution is inducible if and only if there exists a non-negative matrix $p$ with dimensions equal to the number of states 
and the number of posterior belief vectors, respectively, which satisfies a particular system of linear equations. In particular, the set of matrices that satisfy these equations is a convex polytope, which implies that the set of language independent communication strategies that induce a given distribution over posterior belief vectors is a convex polytope as well.

We next explore the informativeness of different communication strategies which induce the same distribution of posterior beliefs: the message a receiver obtains reveals not only information about the true state of the world, but also about the information that other receivers have. Let's return to our operative who wants to create chaos in a foreign country. If one receiver knew (i.e., believes with probability 1) that another receiver knew whether the true state is red or blue, he might decide not to engage in an argument at all. Thus, our operative might want to reveal as little information as possible to any receiver about what other receivers know. As an example suppose that before the operative engages, two receivers believe that either state might be true with probability $1 / 2$. Suppose the operative engages in private communication with both and sends signals as follows.

\begin{tabular}{c|ccc}
$\pi^{\prime}$ & $(m, r)$ & $(m, b)$ & $(x, x)$ \\
\hline Red & $\frac{1}{2}$ & 0 & $\frac{1}{2}$ \\
Blue & 0 & $\frac{1}{2}$ & $\frac{1}{2}$
\end{tabular}

In this case receiver 2 knows that the true state is red if he observes $r$, he knows the true state is blue if he observes $b$, and he learns nothing if he observes $x$. Agent 1 never learns anything about the true state. If he observes $m$, however, he knows that receiver 2 knows the true state. If the sender would replace $m$ by $x$, receiver 1 would not learn anything at all.

This example illustrates that a receiver's knowledge about the true state and the signal realization can differ among communication strategies, even if the latter induce identical distributions over posterior belief vectors. In particular, a receiver may have different knowledge about another receiver's knowledge about the true state and the signal realization. It is then natural to ask what types of communication strategies that induce the same distribution restrict this knowledge the most. In the example above, different messages might lead to the same posterior belief but to different higher order knowledge. By employing direct or even language independent communication strategies we could avoid this issue. But even then: not all language independent communication strategies reveal the same amount of information. To make this more precise, we define information correspondences that describe what receivers know about the true state and the true posterior belief vector (instead of the signal realization), where we call a tuple of a state and a posterior belief vector a posterior history. A communication strategy is more informative than another if for every receiver, every state, and every signal that can occur in this state, the set of posterior histories that the receiver deems possible is smaller under the former than under the latter. We prove that for any inducible distribution over posterior belief vectors the least informative communication strategies that 
induce it lie in the relative interior of the set of all language independent communication strategies that induce it.

The rest of the paper is organized as follows. In Section 4.2 we discuss related literature. In Section 4.3 we provide preliminary definitions and results. We then characterize sets of belief vectors that can be a subset of the support of an inducible distribution over posterior belief vectors in Section 4.4. In Section 4.5 we introduce minimal and direct communication strategies, and in Section 4.6 we turn to language independent communication strategies. In Section 4.7 we characterize inducible distributions of posteriors and provide several implications. Section 4.8 introduces information and posterior correspondences. In Section 4.9 we explore the informativeness of communication strategies and we conclude in Section 4.10. All proofs can be found in Appendix 4.11.

\subsection{Related Literature}

Regarding the part of the paper where we focus on inducible distributions of posteriors, one close study to ours is Arieli et al. (2020). They consider multiple receivers who share a common prior belief on a binary state space and study joint posterior belief distributions. They first show that for the case of two receivers a quantitative version of the Agreement Theorem of $\mathrm{Au}-$ mann (1976) holds; beliefs of receivers are approximately equal when they are approximately common knowledge. For more than two receivers, they relate the feasibility condition to the No Trade Theorem of Milgrom and Stokey (1982) and provide a characterization of feasible joint posteriors. These characterizations are then applied to study independent joint posterior belief distributions. While we pose the same question as Arieli et al. (2020), we obtain a completely different characterization while allowing for an arbitrary finite state space. Another related paper is Ziegler (2019), which follows a similar approach to Arieli et al. (2020). While the author also provides a characterization of feasible joint posteriors, Arieli et al. (2020) show that the necessary and sufficient condition provided by Ziegler (2019) becomes insufficient if the support of the marginal distributions contains more than two points.

Like Arieli et al. (2020) and Ziegler (2019) we provide a characterization of inducible distributions over posterior belief vectors. Mathevet et al. (2020) focus instead on inducible distributions over belief hierarchies. Their characterization requires Bayes plausibility at the level of the sender and formulates two equations to obtain the correct belief hierarchies of the receivers. A central concept in their characterization is sender's belief about the state given the entire hierarchy profile. Our central tool is in terms of a matrix with dimensions given by the number of states and the number of posterior belief vectors.

While we focus on inducible distributions of posterior belief vectors, Bergemann and Morris (2016a) consider a game-theoretic set-up and study the distributions of receivers' actions that 
sender can induce, more precisely they characterize the set of Bayes correlated equilibria of the game. An advantage of their approach is that there is no need to make explicit use of information structures. They also develop an extension of the classic sufficiency condition of Blackwell (1953) for the multi-player set-up and show that more information according to that criterion results in a smaller set of Bayes correlated equilibria. A similar set-up is studied by Taneva (2019), who derives sender's optimal information structure.

In the single receiver case, introducing heterogeneity may render Bayes plausibility insufficient for a distribution to be inducible. Alonso and Camara (2016) consider a single receiver who does not share a common prior with the sender and show that an additional condition is required on top of Bayes plausibility. Beauchêne et al. (2019) also consider a single receiver, who is ambiguity averse, and a sender who may use an ambiguous communication device. In that case they are able to show that a modified version of Bayes plausibility holds. When there are multiple receivers, if information is perfectly correlated, then Bayes plausibility is still the only condition for inducibility since in this case all receivers have the same ex-post belief. The first part of Wang (2013) and Alonso and Câmara (2016) both consider public communication and are examples of such a situation.

There is a wide literature that focuses on informativeness in the sense of Blackwell (1953). ${ }^{2}$ Rick (2013) considers an informed sender and an uninformed receiver and shows that miscommunication expands the set of distributions of beliefs the sender expects to induce. Gentzkow and Kamenica (2016) consider multiple senders and a single receiver and show that the amount of revealed information increases with the number of senders. Ichihashi (2019) considers a model of a single sender and receiver in which a designer can restrict the most informative signal that the sender can generate, and he characterizes the information restriction that maximizes the receiver's payoff. While these papers compare the informativeness of different information structures by investigating the induced distributions of posteriors, we analyze informativeness according to the higher order knowledge a receiver has about the posterior history.

\subsection{Preliminaries and Notation}

Let $N=\{1, \ldots, n\}$ be the set of receivers and $\Omega$ be a finite set of states of the world. For any set $X$ denote by $\Delta(X)$ the set of probability distributions over $X$ with finite support. For finite $X$ denote by $\Delta^{\circ}(X)$ the set of probability distribution over $X$ with full support. We assume that sender and receivers share a common prior belief $\lambda^{0} \in \Delta^{\circ}(\Omega)$.

Let $S_{i}$ be a non-empty set of messages sender can send to receiver $i \in N$, and let $S=$

\footnotetext{
${ }^{2} \mathrm{Li}(2017)$ considers a different criterion and measures informativeness in the sense of Ganuza and Penalva
} (2010), where more informative signals lead to greater variability of conditional expectations. 
$\prod_{i \in N} S_{i}$. The elements of $S$ are called signals. A communication strategy is a function $\pi: \Omega \rightarrow \Delta(S)$ that maps each $\omega \in \Omega$ to a finite probability distribution over $S$. The set of possible signal realizations is denoted by $S^{\pi}=\{s \in S \mid \exists \omega \in \Omega: \pi(s \mid \omega)>0\}$. Note that receiver $i \in N$ knows the joint distributions $\pi(\cdot \mid \omega)$ for all $\omega \in \Omega$, but only observes his private message $s_{i}$ when signal $s$ realizes. Denote the set of all communication strategies by $\Pi$. For each $\pi \in \Pi, s_{i} \in S_{i}$, and $\omega \in \Omega$, let

$$
\pi_{i}\left(s_{i} \mid \omega\right)=\sum_{t \in S: t_{i}=s_{i}} \pi(t \mid \omega)
$$

which is the probability that receiver $i \in N$ observes $s_{i}$ given that the true state is $\omega$. For each $i \in N$, define $S_{i}^{\pi}=\left\{s_{i} \in S_{i} \mid \exists \omega \in \Omega: \pi_{i}\left(s_{i} \mid \omega\right)>0\right\}$, which is the set of messages receiver $i$ observes with positive probability under $\pi$.

Given a communication strategy $\pi \in \Pi$, a signal $s \in S^{\pi}$ generates the posterior belief vector $\lambda^{s} \in \Delta(\Omega)^{n}$ defined by

$$
\lambda_{i}^{s}(\omega)=\frac{\pi_{i}\left(s_{i} \mid \omega\right) \lambda^{0}(\omega)}{\sum_{\omega^{\prime} \in \Omega} \pi_{i}\left(s_{i} \mid \omega^{\prime}\right) \lambda^{0}\left(\omega^{\prime}\right)}, \quad i \in N, \omega \in \Omega .
$$

So, $\lambda_{i}^{s}(\omega)$ is $i$ 's posterior belief that the true state is $\omega$ upon receiving message $s_{i}$.

A communication strategy $\pi \in \Pi$ induces the distribution $\sigma \in \Delta\left(\Delta(\Omega)^{n}\right)$ over posterior belief vectors if for all $\lambda \in \Delta(\Omega)^{n}$ it holds that

$$
\sigma(\lambda)=\sum_{s \in S^{\pi}: \lambda^{s}=\lambda} \sum_{\omega \in \Omega} \pi(s \mid \omega) \lambda^{0}(\omega) .
$$

In words, $\sigma(\lambda)$ is the probability of posterior belief vector $\lambda$. The distribution over posterior belief vectors induced by $\pi$ is denoted by $\sigma^{\pi}$. We define the set of inducible distributions over posterior belief vectors by

$$
\Sigma=\left\{\sigma \in \Delta\left(\Delta(\Omega)^{n}\right) \mid \exists \pi \in \Pi \text { such that } \sigma^{\pi}=\sigma\right\}
$$

Observe that $\Sigma$ depends on the set $S$ of signals that the sender can use: a distribution $\sigma$ might only be inducible if $S$ is sufficiently rich. This becomes relevant in situations where the sender's signal space is a priori limited, be it in case of schools who are bound to reveal information about students' qualities within a grading framework (Boleslavsky and Cotton, 2015), or in case of a regulator who can reveal information about a bank's financial situation only by a simple pass/fail stress test (Inostrozosa and Pavan, 2020). Thus, we will provide necessary and sufficient conditions on the size of $S$ whenever appropriate.

We denote the support of $\sigma \in \Delta\left(\Delta(\Omega)^{n}\right)$ by $R(\sigma)$. By our assumptions made so far, the support of $\sigma$ is a finite set. For each $i \in N$ and $\lambda_{i} \in \Delta(\Omega)$, define

$$
\sigma_{i}\left(\lambda_{i}\right)=\sum_{\lambda^{\prime} \in R(\sigma): \lambda_{i}^{\prime}=\lambda_{i}} \sigma\left(\lambda^{\prime}\right) .
$$


That is, $\sigma_{i}\left(\lambda_{i}\right)$ is the probability that receiver $i$ has posterior belief $\lambda_{i}$. We denote the support of $\sigma_{i}$ by $R\left(\sigma_{i}\right)$.

Let $\sigma, \sigma^{\prime} \in \Delta\left(\Delta(\Omega)^{n}\right)$ be two distributions over posterior belief vectors and let $\alpha \in[0,1]$. The convex combination $\hat{\sigma}=\alpha \sigma+(1-\alpha) \sigma^{\prime}$ is defined by

$$
\hat{\sigma}(\lambda)=\alpha \sigma(\lambda)+(1-\alpha) \sigma^{\prime}(\lambda), \quad \lambda \in \Delta(\Omega)^{n}
$$

Even in the case with a single receiver, $\Sigma$ need not be convex. For instance, if $S$ consists of two messages, then it is possible to induce $\sigma, \sigma^{\prime} \in \Sigma$ with disjoint supports of cardinality 2 . If $\hat{\sigma}$ is a strict convex combination of $\sigma$ and $\sigma^{\prime}$, then $|\hat{\sigma}|=4$, so that $\hat{\sigma}$ cannot be induced with two messages only. The next result shows that $\Sigma$ is convex when the signal space is sufficiently rich.

Proposition 4.1. Let $\sigma, \sigma^{\prime} \in \Sigma$ and $\alpha \in(0,1)$. Then $\alpha \sigma+(1-\alpha) \sigma^{\prime} \in \Sigma$ if and only if $\left|S_{i}\right| \geq\left|R\left(\sigma_{i}\right) \cup R\left(\sigma_{i}^{\prime}\right)\right|$ for all $i \in N$.

Most of the literature considers $S_{i}$ an arbitrary set that contains all messages that are necessary. The previous proposition implies that in this case the set of inducible posteriors is convex.

A distribution over posterior belief vectors $\sigma \in \Delta\left(\Delta(\Omega)^{n}\right)$ is Bayes plausible if

$$
\sum_{\lambda_{i} \in R\left(\sigma_{i}\right)} \lambda_{i}(\omega) \sigma_{i}\left(\lambda_{i}\right)=\lambda^{0}(\omega), \quad i \in N, \omega \in \Omega
$$

That is, for each receiver the expected posterior belief equals his prior belief. Kamenica and Gentzkow (2011) show that $\Sigma$ is the set of Bayes plausible posterior distributions in the single receiver case, given that $S$ is sufficiently rich. It now follows for the multiple receiver case that every $\sigma \in \Sigma$ satisfies Bayes plausibility. We therefore obtain the following result, which is stated for later reference and without proof.

Proposition 4.2. Every $\sigma \in \Sigma$ is Bayes plausible.

\subsection{Implementing Belief Vectors}

When a sender is interacting with a single receiver who has no private information, Bayes plausibility of a distribution $\sigma \in \Delta\left(\Delta(\Omega)^{n}\right)$ is necessary and sufficient for $\sigma$ to belong to $\Sigma$ when $S$ is sufficiently rich. In particular, for any $\lambda \in \Delta(\Omega)$ there is $\sigma \in \Sigma$ such that $\sigma(\lambda)>0$. In contrast, in the multiple receiver case it is not true that any single posterior belief vector $\lambda \in \Delta(\Omega)^{n}$ can occur with positive probability for a suitably chosen communication strategy. Our first proposition shows that $\lambda \in \Delta(\Omega)^{n}$ can belong to the support of some $\sigma \in \Sigma$ if and only if there is at least one state which, according to $\lambda$, is deemed possible by all receivers. 
Proposition 4.3. For every $i \in N$, let $S_{i}$ contain at least two messages. Let $\lambda \in \Delta(\Omega)^{n}$. There exists $\sigma \in \Sigma$ with $\sigma(\lambda)>0$ if and only if there is $\omega \in \Omega$ such that $\prod_{i \in N} \lambda_{i}(\omega)>0$.

Let there be two receivers and a binary state space, say $\Omega=\{X, Y\}$, as in our example in the introduction. It follows from Proposition 4.3 that a posterior belief vector $\lambda$ with $\lambda(X)=(0,1)$ cannot result with positive probability under any communication protocol since $\lambda_{1}(X) \lambda_{2}(X)=0$ and $\lambda_{1}(Y) \lambda_{2}(Y)=0$. At the same time, for each $\varepsilon>0$, the posterior belief vector $\lambda$ with $\lambda(X)=(\varepsilon, 1)$ can be obtained with positive probability.

We now generalize Proposition 4.3 from a single posterior belief vector to finite sets of posterior belief vectors.

Proposition 4.4. Let $R \subseteq \Delta(\Omega)^{n}$ be finite. For every $i \in N$, let $S_{i}$ contain at least $\left|R_{i}\right|+1$ messages, where $R_{i}=\left\{\lambda_{i} \in \Delta(\Omega) \mid \lambda \in R\right\}$. There exists $\sigma \in \Sigma$ with $R \subseteq R(\sigma)$ if and only if for each $\lambda \in R$ there exists $\omega \in \Omega$ such that $\prod_{i \in N} \lambda_{i}(\omega)>0$.

At this point we have identified sets that can be subsets of the support of an inducible distribution over posterior belief vectors. In Section 4.7 we characterize all inducible distributions over posterior belief vectors and the sets that can be the support of such distributions.

\subsection{Minimal and Direct Communication Strategies}

A large part of the literature is interested in "straightforward" communication strategies (Kamenica and Gentzkow, 2011) that send recommendations to receivers about what action to take. In the present paper, we do not specify sets of feasible actions for receivers, so that sending recommendations has no meaning. Nevertheless, some communication strategies are easier to handle than others and this and the next section will introduce some important classes.

Given a communication strategy $\pi \in \Pi$ and $s, s^{\prime} \in S^{\pi}$ with $s \neq s^{\prime}$, it is possible that $\lambda^{s}=\lambda^{s^{\prime}}$. That is, two distinct signals can generate the same posterior belief vector. This motivates the following definition.

Definition 4.5. A communication strategy $\pi \in \Pi$ is minimal if $\left|S^{\pi}\right|=\left|R\left(\sigma^{\pi}\right)\right|$. The set of minimal communication strategies is denoted by $\Pi^{\mathrm{m}}$.

Under a minimal communication strategy, different signals lead to different posterior belief vectors. We give an illustration of a minimal communication strategy in the following example.

Example 4.6. Let $N=\{1,2\}, \Omega=\{X, Y\}, S_{1}=\{v, w\}$, and $S_{2}=\{w, x, y\}$. Assume that agents have a common prior $\lambda^{0}(X)=1 / 2$. Let $\pi$ be given as follows: 


\begin{tabular}{c|ccc}
$\pi$ & $(v, x)$ & $(v, y)$ & $(w, w)$ \\
\hline$X$ & $\frac{1}{2}$ & 0 & $\frac{1}{2}$ \\
$Y$ & 0 & $\frac{1}{2}$ & $\frac{1}{2}$
\end{tabular}.

We have $S^{\pi}=\{(v, x),(v, y),(w, w)\}$. Irrespective of the signal received, receiver 1 gathers no information about the state: he has posterior beliefs $\lambda_{1}^{(v, x)}(X)=\lambda_{1}^{(v, y)}(X)=\lambda_{1}^{(w, w)}(X)=1 / 2$. For receiver 2, we have $\lambda_{2}^{(v, x)}(X)=1, \lambda_{2}^{(v, y)}(X)=0$, and $\lambda_{2}^{(w, w)}(X)=1 / 2$. It follows that

$$
R\left(\sigma^{\pi}\right)=\{((1 / 2,1 / 2),(1,0)),((1 / 2,1 / 2),(0,1)),((1 / 2,1 / 2),(1 / 2,1 / 2))\} .
$$

Since $\left|S^{\pi}\right|=\left|R\left(\sigma^{\pi}\right)\right|, \pi$ is minimal.

In case of a single receiver, it is sufficient to have a bijection between $S^{\pi}$ and $R(\sigma)$ to ensure that each message leads to a different posterior, that is, to ensure that the communication strategy employs a minimal number of messages. If there are multiple receivers, however, the existence of such a bijection does not guarantee that the number of messages for each receiver is indeed minimal. For instance, the two messages $v, w$ in Example 4.5 both lead to the posterior belief $\lambda_{1}(X)=1 / 2$ for receiver 1 .

Definition 4.7. A communication strategy $\pi \in \Pi$ is direct if for all $i \in N$ it holds that $\left|S_{i}^{\pi}\right|=\left|R\left(\sigma_{i}^{\pi}\right)\right|$. The set of direct communication strategies is denoted by $\Pi^{\mathrm{d}}$.

Under a direct communication strategy any two different messages must lead to two different posterior beliefs. Hence, the number of different posterior beliefs a receiver can have equals the cardinality of $S_{i}^{\pi}$.

Example 4.8. Recall the minimal communication strategy $\pi$ in Example 4.6. Receiver 1 has the same posterior belief after observing $v$ and observing $w$, i.e., $\lambda_{1}^{(v, x)}(X)=\lambda_{1}^{(w, w)}(X)$. Thus, $\pi$ is not direct. Consider the communication strategy $\pi^{\prime}$ defined by:

\begin{tabular}{c|ccc}
$\pi^{\prime}$ & $(w, x)$ & $(w, y)$ & $(w, w)$ \\
\hline$X$ & $\frac{1}{2}$ & 0 & $\frac{1}{2}$ \\
$Y$ & 0 & $\frac{1}{2}$ & $\frac{1}{2}$
\end{tabular}.

We have $S^{\pi^{\prime}}=\{(w, x),(w, y),(w, w)\}$ and accordingly we can write the support of $\sigma^{\pi^{\prime}}$ as

$$
R\left(\sigma^{\pi^{\prime}}\right)=\{((1 / 2,1 / 2),(1,0)),((1 / 2,1 / 2),(0,1)),((1 / 2,1 / 2),(1 / 2,1 / 2))\} .
$$

Note that $R\left(\sigma^{\pi}\right)=R\left(\sigma^{\pi^{\prime}}\right)$. Since for all $s, t \in S^{\pi^{\prime}}$ and each $i \in N$ we have $\lambda_{i}^{\prime s}=\lambda_{i}^{\prime t}$ if and only if $s_{i}=t_{i}, \pi^{\prime}$ is direct.

For any communication strategy $\pi \in \Pi,\left|S_{i}^{\pi}\right|=\left|R\left(\sigma_{i}^{\pi}\right)\right|$ guarantees that a minimal number of messages is employed and implies that the number of employed signals is minimal as well. Thus, the following lemma does not come as a surprise. 
Lemma 4.9. It holds that $\Pi^{\mathrm{d}} \subseteq \Pi^{\mathrm{m}}$.

We close this section by claiming that any distribution in $\Sigma$ can be induced by a direct communication strategy. In Appendix 4.11, we provide the proofs of some of the results that appear later than Theorem 4.10 in the text before the proof of Theorem 4.10, as it will then follow easily.

Theorem 4.10. If $\sigma \in \Sigma$, then there exists $\pi \in \Pi^{\mathrm{d}}$ such that $\sigma^{\pi}=\sigma$.

\subsection{Language Independent Communication Strategies}

The same distribution over posterior belief vectors can be induced by various communication strategies with potentially disjoint signal spaces. We now proceed to show that there is a canonical way to describe communication strategies. The principal idea is that the sender sends to each receiver the belief that he should have after observing the message.

Definition 4.11. A communication strategy $\pi \in \Pi$ is a language independent communication strategy (LICS) if $S^{\pi} \subseteq \Delta(\Omega)^{n}$ and, for all $s \in S^{\pi}, \lambda^{s}=s$. The set of language independent communication strategies is denoted by $\Pi^{\ell}$.

Example 4.12. Let $N=\{1,2\}, \Omega=\{X, Y\}$, and $\lambda^{0}(X)=1 / 3$. The communication strategy $\pi \in \Pi$ is defined as follows:

\begin{tabular}{c|cccc}
$\pi$ & $(x, x)$ & $(x, y)$ & $(y, x)$ & $(y, y)$ \\
\hline$X$ & $\frac{1}{4}$ & $\frac{1}{4}$ & $\frac{1}{4}$ & $\frac{1}{4}$ \\
$Y$ & $\frac{1}{8}$ & $\frac{1}{8}$ & $\frac{1}{8}$ & $\frac{5}{8}$
\end{tabular}.

For any $i \in N$, we have $\lambda_{i}^{(x, x)}(X)=1 / 2$ and $\lambda_{i}^{(y, y)}(X)=1 / 4$. Hence, $\pi$ is in fact direct. The support of $\sigma^{\pi}$ is equal to

$$
\begin{aligned}
R\left(\sigma^{\pi}\right) & =\left\{\lambda^{(x, x)}, \lambda^{(x, y)}, \lambda^{(y, x)}, \lambda^{(y, y)}\right\} \\
& =\left\{\left(\left(\frac{1}{2}, \frac{1}{2}\right),\left(\frac{1}{2}, \frac{1}{2}\right)\right)\left(\left(\frac{1}{2}, \frac{1}{2}\right),\left(\frac{1}{4}, \frac{3}{4}\right)\right),\left(\left(\frac{1}{4}, \frac{3}{4}\right),\left(\frac{1}{2}, \frac{1}{2}\right)\right),\left(\left(\frac{1}{4}, \frac{3}{4}\right),\left(\frac{1}{4}, \frac{3}{4}\right)\right)\right\} .
\end{aligned}
$$

It holds that $\sigma^{\pi}\left(\lambda^{(x, x)}\right)=\sigma^{\pi}\left(\lambda^{(x, y)}\right)=\sigma^{\pi}\left(\lambda^{(y, x)}\right)=1 / 6$ and $\sigma^{\pi}\left(\lambda^{(y, y)}\right)=1 / 2$.

The communication strategy $\pi^{\prime} \in \Pi$ is obtained by switching messages $x$ and $y$, so

\begin{tabular}{c|cccc}
$\pi^{\prime}$ & $(x, x)$ & $(x, y)$ & $(y, x)$ & $(y, y)$ \\
\hline$X$ & $\frac{1}{4}$ & $\frac{1}{4}$ & $\frac{1}{4}$ & $\frac{1}{4}$ \\
$Y$ & $\frac{5}{8}$ & $\frac{1}{8}$ & $\frac{1}{8}$ & $\frac{1}{8}$
\end{tabular}.

It is immediate that $\sigma^{\pi}=\sigma^{\pi^{\prime}}$.

Next, consider the communication strategy $\hat{\pi}$ that corresponds to the convex combination of $\pi$ and $\pi^{\prime}$ with equal weights: $\hat{\pi}=1 / 2 \pi+1 / 2 \pi^{\prime}$. We have that 


\begin{tabular}{c|cccc}
$\hat{\pi}$ & $(x, x)$ & $(x, y)$ & $(y, x)$ & $(y, y)$ \\
\hline$X$ & $\frac{1}{4}$ & $\frac{1}{4}$ & $\frac{1}{4}$ & $\frac{1}{4}$ \\
$Y$ & $\frac{3}{8}$ & $\frac{1}{8}$ & $\frac{1}{8}$ & $\frac{3}{8}$
\end{tabular}.

Perhaps surprisingly, it holds that $\sigma^{\hat{\pi}} \neq \sigma^{\pi}=\sigma^{\pi^{\prime}}{ }^{3}$ It is easily verified that $\sigma^{\hat{\pi}}$ is the distribution that assigns probability 1 to the posterior belief vector $\left(\lambda^{0}, \lambda^{0}\right)$. It follows that the set of communication strategies which induce a particular distribution is not convex. Observe that $\hat{\pi}$ is not direct, which implies that $\Pi^{\mathrm{d}}$ is also not convex.

The communication strategies $\pi^{\ell}, \pi^{\prime \ell}$, and $\hat{\pi}^{\ell}$ are obtained by relabeling the signals sent by $\pi, \pi^{\prime}$, and $\hat{\pi}$, respectively, with the posterior belief vectors they lead to. We have that $\pi^{\ell}=\pi^{\prime \ell}$. Both are equal to

\begin{tabular}{c|cccc}
$\pi^{\ell}, \pi^{\prime \ell}$ & $\left(\left(\frac{1}{2}, \frac{1}{2}\right),\left(\frac{1}{2}, \frac{1}{2}\right)\right)$ & $\left(\left(\frac{1}{2}, \frac{1}{2}\right),\left(\frac{1}{4}, \frac{3}{4}\right)\right)$ & $\left(\left(\frac{1}{4}, \frac{3}{4}\right),\left(\frac{1}{2}, \frac{1}{2}\right)\right)$ & $\left(\left(\frac{1}{4}, \frac{3}{4}\right),\left(\frac{1}{4}, \frac{3}{4}\right)\right)$ \\
\hline$X$ & $\frac{1}{4}$ & $\frac{1}{4}$ & $\frac{1}{4}$ & $\frac{1}{4}$ \\
$Y$ & $\frac{1}{8}$ & $\frac{1}{8}$ & $\frac{1}{8}$ & $\frac{5}{8}$
\end{tabular}.

Each receiver has posterior belief $(1 / 2,1 / 2)$ upon observing message $(1 / 2,1 / 2)$ and has posterior belief $(1 / 4,3 / 4)$ upon observing message $(1 / 4,3 / 4)$. Thus, $\pi^{\ell}$ and $\pi^{\prime \ell}$ are language independent communication strategies.

Finally, $\hat{\pi}^{\ell}$ sends $\lambda^{0}$ to both players with probability 1. In particular, $\hat{\pi}^{\ell}$ is not a convex combination of $\pi^{\ell}$ and $\pi^{\prime} \ell$.

The next result states that an LICS is direct.

Lemma 4.13. It holds that $\Pi^{\ell} \subseteq \Pi^{\mathrm{d}}$.

By Lemma 4.13 we know that an LICS is direct and by Lemma 4.9 directness implies minimality. Thus, there is a chain of inclusions between $\Pi^{\ell}, \Pi^{\mathrm{d}}$, and $\Pi^{\mathrm{m}}$.

Corollary 4.14. It holds that $\Pi^{\ell} \subseteq \Pi^{\mathrm{d}} \subseteq \Pi^{\mathrm{m}} \subseteq \Pi$.

Since we can transform any given direct communication strategy into an LICS by relabeling each message with the posterior belief that message leads to, an immediate consequence of Theorem 4.10 is that any element of $\Sigma$ can be induced by an LICS if $\Delta(\Omega)^{n} \subseteq S$, a result also obtained by Arieli et al. (2020) for a binary state space. One advantage of language independent communication strategies is that for each $\sigma \in \Sigma$ the set of all language independent communication strategies that induce $\sigma$, denoted by $\Pi^{\ell}(\sigma)$, is convex. The proof of this statement, however, is postponed as it follows easily from later results.

\footnotetext{
${ }^{3}$ Observe that this is no contradiction to the proof of Proposition 4.1: there we used that any fixed message induces under ever communication strategy where it is sent with positive probability the same posterior. Here, message $x$ induces posterior $(1 / 2,1 / 2)$ under $\pi$ but $(1 / 4,3 / 4)$ under $\pi^{\prime}$.
} 
Proposition 4.15. Let $\Delta(\Omega)^{n} \subseteq S$ and $\sigma \in \Sigma$. Then $\Pi^{\ell}(\sigma)$ is non-empty and convex.

Proposition 4.15 contrasts Example 4.12 where we showed that both the set of all communication strategies and the set of all direct communication strategies that induce a given $\sigma$ are typically not convex. This makes language independent communication strategies particularly attractive.

Recall that given a direct communication strategy, we can obtain an LICS by simply replacing messages with the posterior beliefs they lead to. More generally, given a communication strategy $\pi \in \Pi$, one can define $\pi^{\prime} \in \Pi$ by a one-to-one change in the names of messages in $S_{i}^{\pi}$ for each $i \in N$. In this case, we typically have $S^{\pi^{\prime}} \neq S^{\pi}$, though we intuitively think of both communication strategies as equivalent. More formally, we have the following definition.

Definition 4.16. Two communication strategies $\pi: \Omega \rightarrow \Delta(S)$ and $\hat{\pi}: \Omega \rightarrow \Delta(\hat{S})$ are equivalent $(\pi \sim \hat{\pi})$ if for every $i \in N$ there is a bijection $\psi_{i}: S_{i}^{\pi} \rightarrow \hat{S}_{i}^{\hat{\pi}}$ such that, for every $\omega \in \Omega$, for every $s \in S^{\pi}, \hat{\pi}(\psi(s) \mid \omega)=\pi(s \mid \omega)$.

We can interpret equivalent communication strategies as providing the same information in different languages. Indeed, let $s_{i} \in S_{i}^{\pi}$ and $\hat{s}_{i} \in \hat{S}_{i}^{\hat{\pi}}$ be such that $\psi_{i}\left(s_{i}\right)=\hat{s}_{i}$. It holds that

$$
\pi_{i}\left(s_{i} \mid \omega\right)=\sum_{t \in S^{\pi}: t_{i}=s_{i}} \pi(t \mid \omega)=\sum_{t \in S^{\pi}: t_{i}=s_{i}} \hat{\pi}(\psi(t) \mid \omega)=\sum_{\hat{t} \in \hat{S}^{\hat{\pi}}: \hat{t}_{i}=\hat{s}_{i}} \hat{\pi}(\hat{t} \mid \omega)=\hat{\pi}_{i}\left(\hat{s}_{i} \mid \omega\right), \quad \omega \in \Omega .
$$

Now consider $s \in S^{\pi}$ and $\hat{s} \in \hat{S}^{\hat{\pi}}$ such that $\hat{s}=\psi(s)$. For every $i \in N$, we have that

$$
\lambda_{i}^{s}(\omega)=\frac{\pi_{i}\left(s_{i} \mid \omega\right) \lambda^{0}(\omega)}{\sum_{\omega^{\prime} \in \Omega} \pi_{i}\left(s_{i} \mid \omega^{\prime}\right) \lambda^{0}\left(\omega^{\prime}\right)}=\frac{\hat{\pi}_{i}\left(\hat{s}_{i} \mid \omega\right) \lambda^{0}(\omega)}{\sum_{\omega^{\prime} \in \Omega} \hat{\pi}_{i}\left(\hat{s}_{i} \mid \omega^{\prime}\right) \lambda^{0}\left(\omega^{\prime}\right)}=\hat{\lambda}_{i}^{\hat{s}}(\omega)
$$

It follows from (4.5) that sending signal $s$ under communication strategy $\pi$ and sending signal $\hat{s}$ under communication strategy $\hat{\pi}$ results in the same posterior belief vector. It is also immediate from Definition 4.16 that $\hat{S}^{\hat{\pi}}=\psi\left(S^{\pi}\right)$.

The next proposition, stating that equivalent communication strategies induce the same distribution over posterior belief vectors, now follows easily.

Proposition 4.17. Let $\pi: \Omega \rightarrow \Delta(S)$ and $\hat{\pi}: \Omega \rightarrow \Delta(\hat{S})$ be such that $\pi \sim \hat{\pi}$. It holds that $\sigma^{\pi}=\sigma^{\hat{\pi}}$.

Note that the converse of Proposition 4.17 is not true: as we will see in Example 4.26 there are communication strategies that induce the same distribution over posterior belief vectors but that are not equivalent.

The next proposition makes clear that each set of equivalent communication strategies contains at most one LICS.

Proposition 4.18. Let $\pi, \pi^{\prime} \in \Pi^{\ell}$ with $\pi \sim \pi^{\prime}$. It holds that $\pi=\pi^{\prime}$. 
Observe that a communication strategy that is not direct cannot be equivalent to an LICS as the required bijection between message spaces cannot exist. Nevertheless for every communication strategy there is a canonical way to find an LICS that induces the same posterior. The construction heavily lies on the following lemma, which is straightforward and therefore stated without proof. ${ }^{4}$

Lemma 4.19. Let $\pi \in \Pi$ be a communication strategy. It holds that

$$
\frac{\sum_{s_{i} \in S_{i}^{\pi}: \lambda_{i}^{s}=\lambda_{i}} \pi_{i}\left(s_{i} \mid \omega\right) \lambda^{0}(\omega)}{\sum_{\omega^{\prime} \in \Omega} \sum_{s_{i} \in S_{i}^{\pi}: \lambda_{i}^{s}=\lambda_{i}} \pi_{i}\left(s_{i} \mid \omega^{\prime}\right) \lambda^{0}\left(\omega^{\prime}\right)}=\lambda_{i}(\omega), \quad \omega \in \Omega, i \in N, \lambda_{i} \in R\left(\sigma_{i}^{\pi}\right) .
$$

Lemma 4.19 extends the formula for Bayesian updating and applies it to all messages simultaneously that lead to a particular posterior belief. According to the lemma, distinct messages that lead to the same posterior can be replaced by the same message. Thus, the following result is immediate and we present it without proof.

Theorem 4.20. Let $\Delta(\Omega)^{n} \subseteq S$. For $\pi \in \Pi$ define $\pi^{\ell}: \Omega \rightarrow \Delta(S)$ as

$$
\pi^{\ell}(\lambda \mid \omega)=\sum_{s \in S^{\pi}: \lambda^{s}=\lambda} \pi(s \mid \omega), \quad \omega \in \Omega, \lambda \in R\left(\sigma^{\pi}\right) .
$$

Then $\sigma^{\pi^{\ell}}=\sigma^{\pi}$. Moreover, if $\pi \in \Pi^{d}$ then $\pi^{\ell}$ is equivalent to $\pi$.

\subsection{Inducible Distributions}

Unlike the single receiver case, when dealing with multiple receivers Bayes plausibility alone is not sufficient to ensure that a distribution over posterior belief vectors belongs to $\Sigma$.

Example 4.21. Let $N=\{1,2,3\}, \Omega=\{X, Y\}$, and $S=\Delta(\Omega)^{3}$. Assume the agents have common prior $\lambda^{0}(X)=1 / 6$. Let $\lambda^{1}(X)=(1 / 2,1 / 2,0), \lambda^{2}(X)=(1 / 2,0,1 / 2), \lambda^{3}(X)=$ $(0,1 / 2,1 / 2)$, and $\lambda^{4}(X)=(0,0,0)$ and let $\sigma \in \Delta\left(\Delta(\Omega)^{3}\right)$ be given by $\sigma\left(\lambda^{1}\right)=\sigma\left(\lambda^{2}\right)=$ $\sigma\left(\lambda^{3}\right)=1 / 6$ and $\sigma\left(\lambda^{4}\right)=1 / 2$. Then, for each $i \in N$, we have $\sigma_{i}(1 / 2,1 / 2)=1 / 3$ and $\sigma_{i}(0,1)=2 / 3$.

First note that $\sigma$ is Bayes plausible:

$$
\sum_{\lambda_{i} \in R\left(\sigma_{i}\right)} \lambda_{i}(X) \sigma_{i}\left(\lambda_{i}\right)=\frac{1}{2} \cdot \sigma_{i}(1 / 2,1 / 2)+0 \cdot \sigma_{i}(0,1)=\frac{1}{2} \cdot \frac{1}{3}=\frac{1}{6}=\lambda^{0}(X), \quad i \in N .
$$

Suppose that communication strategy $\pi \in \Pi$ induces $\sigma$. By Corollary 4.20 it is without loss of generality to assume that $\pi \in \Pi^{\ell}$. In this case, for any receiver, observing $(1 / 2,1 / 2)$ leads to posterior belief $(1 / 2,1 / 2)$, and observing $(0,1)$ leads to posterior belief $(0,1)$. This implies that receivers cannot observe $(0,1)$ in state $X$, i.e., $\pi_{i}((0,1) \mid X)=0$ for all $i \in N$. It follows that $\pi\left(\lambda^{1} \mid X\right)=\pi\left(\lambda^{2} \mid X\right)=\pi\left(\lambda^{3} \mid X\right)=\pi\left(\lambda^{4} \mid X\right)=0$, which obviously leads to a contradiction. $\triangle$

\footnotetext{
${ }^{4}$ It is implied by the proof of Lemma 3.4 in Kerman et al. (2020).
} 
To guarantee that a distribution over posterior belief vectors belongs to $\Sigma$, additional conditions need to be imposed on top of Bayes plausibility. In Theorem 4.22, we provide necessary and sufficient conditions for a distribution over posterior belief vectors to belong to $\Sigma$.

Theorem 4.22. Let $\sigma \in \Delta\left(\Delta(\Omega)^{n}\right)$ be such that, for every $i \in N,\left|S_{i}\right| \geq\left|R\left(\sigma_{i}\right)\right|$. Then $\sigma \in \Sigma$ if and only if $\sigma$ is Bayes plausible and there exists $p \in \mathbb{R}_{+}^{\Omega \times R(\sigma)}$ such that

(i) $\quad \sum_{\omega \in \Omega} p(\omega, \lambda)=\sigma(\lambda), \quad \lambda \in R(\sigma)$

(ii) $\sum_{\lambda^{\prime} \in R(\sigma): \lambda_{i}^{\prime}=\lambda_{i}} p\left(\omega, \lambda^{\prime}\right)=\lambda_{i}(\omega) \sigma_{i}\left(\lambda_{i}\right), \omega \in \Omega, i \in N, \lambda_{i} \in R\left(\sigma_{i}\right)$.

If $\sigma \in \Sigma$, then the communication strategy $\pi: \Omega \rightarrow \Delta\left(\Delta(\Omega)^{n}\right)$ defined by

$$
\pi(\lambda \mid \omega)=\frac{p(\omega, \lambda)}{\lambda^{0}(\omega)}, \quad \omega \in \Omega, \lambda \in R(\sigma),
$$

is an LICS such that $\sigma^{\pi}=\sigma$.

Theorem 4.22 makes explicit what is needed in addition to Bayes plausibility to ensure that a distribution over posterior belief vectors belongs to $\Sigma$. Observe that any $p \in \mathbb{R}_{+}^{\Omega \times R(\sigma)}$ which satisfies Condition $(i)$ is a finite probability distribution, that is, $p \in \Delta(\Omega \times R(\sigma))$.

Condition $(i)$ can be interpreted as "posterior marginality" as it states that the probability of a posterior belief vector $\lambda$ is the marginal of $p(\omega, \lambda)$. The right-hand side of condition (ii) is the probability that $\omega$ is the true state according to $i$ 's belief $\lambda_{i}$ multiplied with the probability that $i$ has belief $\lambda_{i}$. Thus, the sum on the left-hand side is the probability that $i$ has belief $\lambda_{i}$ and $\omega$ is the true state.

While Theorem 4.22 is useful in determining whether a distribution of beliefs is inducible, it also provides an LICS that induces the desired distribution. In Example 4.24, we first use Theorem 4.22 to show that a given distribution of beliefs is not inducible. Then, in Example 4.26 , we provide two communication strategies that induce the same distribution via distinct solutions to conditions $(i)$ and $(i i)$.

For any $\sigma \in \Sigma$, define

$$
P(\sigma)=\left\{p \in \mathbb{R}_{+}^{\Omega \times R(\sigma)} \mid p \text { satisfies (i) and (ii) of Theorem } 4.22\right\} .
$$

As $P(\sigma)$ is defined as the set of non-negative matrix solutions to a system of linear equalities, where the system is such that the components of any solution matrix sum up to one, we immediately have the following result.

Corollary 4.23. For every $\sigma \in \Sigma, P(\sigma)$ is a non-empty, compact, and convex polytope.

In the next example, we use Theorem 4.22 to determine whether a given distribution over posterior belief vectors belongs to $\Sigma$. 
Example 4.24. Recall the distribution over posterior belief vectors $\sigma$ in Example 4.21 with

$$
\begin{aligned}
R(\sigma) & =\left\{\lambda^{1}, \lambda^{2}, \lambda^{3}, \lambda^{4}\right\} \\
& =\left\{\left(\left(\frac{1}{2}, \frac{1}{2}\right),\left(\frac{1}{2}, \frac{1}{2}\right),(0,1)\right),\left(\left(\frac{1}{2}, \frac{1}{2}\right),(0,1),\left(\frac{1}{2}, \frac{1}{2}\right)\right),\left((0,1),\left(\frac{1}{2}, \frac{1}{2}\right),\left(\frac{1}{2}, \frac{1}{2}\right)\right),((0,1),(0,1),(0,1))\right\} .
\end{aligned}
$$

Moreover, we have $\sigma\left(\lambda^{1}\right)=\sigma\left(\lambda^{2}\right)=\sigma\left(\lambda^{3}\right)=1 / 6$ and $\sigma\left(\lambda^{4}\right)=1 / 2$.

Suppose $\sigma \in \Sigma$. Then, by Theorem 4.22 there exists $p \in P(\sigma)$ such that

$$
\begin{aligned}
& p\left(X, \lambda^{1}\right)+p\left(X, \lambda^{2}\right)=p\left(X, \lambda^{1}\right)+p\left(X, \lambda^{3}\right)=p\left(X, \lambda^{2}\right)+p\left(X, \lambda^{3}\right)=\frac{1}{6} \\
& p\left(X, \lambda^{1}\right)+p\left(X, \lambda^{4}\right)=p\left(X, \lambda^{2}\right)+p\left(X, \lambda^{4}\right)=p\left(X, \lambda^{3}\right)+p\left(X, \lambda^{4}\right)=0,
\end{aligned}
$$

where we make use of Condition (ii) for $\omega=X$. From the first line we obtain $p\left(X, \lambda^{1}\right)=$ $p\left(X, \lambda^{2}\right)=p\left(X, \lambda^{3}\right)=1 / 12$. Combining this with the second, we find $p\left(X, \lambda^{4}\right)=-1 / 12$. Thus, $p$ fails to be non-negative and $\sigma \notin \Sigma$.

Proposition 4.4 gives a necessary and sufficient condition for a finite set $R \subseteq \Delta(\Omega)^{n}$ to be a subset of $R(\sigma)$ for some $\sigma \in \Sigma$. We will now provide a necessary and sufficient condition for the opposite inclusion, i.e., we characterize those sets $R \subseteq \Delta(\Omega)^{n}$ such that there is some inducible $\sigma \in \Sigma$ whose support is restricted to $R$. We also characterize those sets $R$ such that $R=R(\sigma)$ for some $\sigma \in \Sigma$.

Proposition 4.25. Let the non-empty and finite $R \subseteq \Delta(\Omega)^{n}$ be such that, for every $i \in N$, $\left|S_{i}\right| \geq\left|R_{i}\right|$. There exists $\sigma \in \Sigma$ with $R(\sigma) \subseteq R$ if and only if there is $p \in \mathbb{R}_{+}^{\Omega \times R}$ such that

$$
\sum_{\lambda \in R} p(\omega, \lambda)=\lambda^{0}(\omega), \quad \omega \in \Omega,
$$

(ii) $\sum_{\lambda^{\prime} \in R: \lambda_{i}^{\prime}=\lambda_{i}} p\left(\omega, \lambda^{\prime}\right)=\lambda_{i}(\omega) \sum_{\omega^{\prime} \in \Omega} \sum_{\lambda^{\prime} \in R: \lambda_{i}^{\prime}=\lambda_{i}} p\left(\omega^{\prime}, \lambda^{\prime}\right), \omega \in \Omega, i \in N, \lambda_{i} \in R_{i}$.

If such $p$ exists, then the communication strategy $\pi: \Omega \rightarrow \Delta(R)$ defined by

$$
\pi(\lambda \mid \omega)=\frac{p(\omega, \lambda)}{\lambda^{0}(\omega)}, \quad \omega \in \Omega, \lambda \in R
$$

is an LICS such that $R\left(\sigma^{\pi}\right) \subseteq R$. Moreover, if $p$ is such that, for all $\lambda \in R, \sum_{\omega \in \Omega} p(\lambda, \omega)>0$, then $R\left(\sigma^{\pi}\right)=R$.

As $\pi$ is defined by (4.8), $(i)$ ensures that $\pi(\cdot \mid \omega) \in \Delta(\Omega)^{n}$ for all $\omega \in \Omega$ and $\pi$ is, hence, a communication strategy. Condition $(i i)$ ensures correct belief updating: as before, the lefthand side is the probability that $i$ has belief $\lambda_{i}$ and the true state is $\omega$; the right-hand side is the product of the probability that the state is $\omega$ conditional on $i$ 's having belief $\lambda_{i}$ and the probability that $i$ has belief $\lambda_{i}$.

In our discussion of Proposition 4.17, stating that equivalent communication strategies induce the same distribution, we announced that the converse be not true. We can now easily provide the required counterexample. 
Example 4.26. Let $N=\{1,2\}, \Omega=\{X, Y\}, \lambda^{0}(X)=1 / 3$, and $S=\Delta(\Omega)^{n}$. Consider the distribution $\sigma$ defined by

$$
\begin{aligned}
R\left(\sigma^{\pi}\right) & =\left\{\lambda^{1}, \lambda^{2}, \lambda^{3}, \lambda^{4}\right\} \\
& =\left\{\left(\left(\frac{1}{2}, \frac{1}{2}\right),\left(\frac{1}{2}, \frac{1}{2}\right)\right)\left(\left(\frac{1}{2}, \frac{1}{2}\right),\left(\frac{1}{4}, \frac{3}{4}\right)\right),\left(\left(\frac{1}{4}, \frac{3}{4}\right),\left(\frac{1}{2}, \frac{1}{2}\right)\right),\left(\left(\frac{1}{4}, \frac{3}{4}\right),\left(\frac{1}{4}, \frac{3}{4}\right)\right)\right\},
\end{aligned}
$$

$\sigma\left(\lambda^{1}\right)=\sigma\left(\lambda^{2}\right)=\sigma\left(\lambda^{3}\right)=1 / 6$ and $\sigma\left(\lambda^{4}\right)=1 / 2$. One can easily verify that $p, p^{\prime} \in \mathbb{R}_{+}^{\Omega \times R(\sigma)}$

\begin{tabular}{|c|c|c|c|c|c|c|c|c|c|}
\hline$p(\omega, \lambda)$ & $\lambda^{1}$ & $\lambda^{2}$ & $\lambda^{3}$ & $\lambda^{4}$ & $p^{\prime}(\omega, \lambda)$ & $\lambda^{1}$ & $\lambda^{2}$ & $\lambda^{3}$ & $\lambda^{4}$ \\
\hline$X$ & $\frac{1}{12}$ & $\frac{1}{12}$ & $\frac{1}{12}$ & $\frac{1}{12}$ & $X$ & $\frac{1}{6}$ & 0 & 0 & $\frac{1}{6}$ \\
\hline$Y$ & $\frac{1}{12}$ & $\frac{1}{12}$ & $\frac{1}{12}$ & $\frac{5}{12}$ & $Y$ & 0 & $\frac{1}{6}$ & $\frac{1}{6}$ & $\frac{1}{3}$ \\
\hline
\end{tabular}
defined by

are both solutions to the system of equations in Theorem 4.22. We define $\pi, \pi^{\prime} \in \Pi^{\ell}$ by applying (4.7) to $p$ and $p^{\prime}$, respectively, that is,

\begin{tabular}{c|cccc}
$\pi(\lambda \mid \omega)$ & $\lambda^{1}$ & $\lambda^{2}$ & $\lambda^{3}$ & $\lambda^{4}$ \\
\hline$X$ & $\frac{1}{4}$ & $\frac{1}{4}$ & $\frac{1}{4}$ & $\frac{1}{4}$ \\
$Y$ & $\frac{1}{8}$ & $\frac{1}{8}$ & $\frac{1}{8}$ & $\frac{5}{8}$
\end{tabular}

\begin{tabular}{c|cccc}
$\pi^{\prime}(\lambda \mid \omega)$ & $\lambda^{1}$ & $\lambda^{2}$ & $\lambda^{3}$ & $\lambda^{4}$ \\
\hline$X$ & $\frac{1}{2}$ & 0 & 0 & $\frac{1}{2}$ \\
$Y$ & 0 & $\frac{1}{4}$ & $\frac{1}{4}$ & $\frac{1}{2}$
\end{tabular}

Both $\pi$ and $\pi^{\prime}$ induce $\sigma$. Yet, as $\pi \neq \pi^{\prime}$, Proposition 4.18 implies that $\pi$ and $\pi^{\prime}$ are not equivalent.

\subsection{The Information and Posterior Correspondences}

Our objective in this section is to provide a framework in which we can analyze what receivers know about each other's messages, so that we can later answer the question of how a sender can make sure that receivers know "as little as possible". We follow the standard approach as based on information correspondences, see for instance Osborne and Rubinstein (1994). This approach differs from informativeness in terms of Blackwell (1953) and does not rely on probabilities.

Given a communication strategy $\pi \in \Pi$, we refer to an element $(\omega, s) \in \Omega \times S^{\pi}$ such that $\pi(s \mid \omega)>0$ as a history and to an element $(\omega, \lambda) \in \Omega \times R\left(\sigma^{\pi}\right)$ such that there exists $s \in S^{\pi}$ with $\pi(s \mid \omega)>0$ and $\lambda^{s}=\lambda$ as a posterior history. We denote the sets of histories and posterior histories, respectively, by

$$
\begin{aligned}
H^{\pi} & =\left\{(\omega, s) \in \Omega \times S^{\pi} \mid \pi(s \mid \omega)>0\right\}, \\
\Lambda^{\pi} & =\left\{(\omega, \lambda) \in \Omega \times \Delta(\Omega)^{n} \mid \exists s \in S^{\pi} \text { such that } \pi(s \mid \omega)>0 \text { and } \lambda^{s}=\lambda\right\} .
\end{aligned}
$$

Note that if $\pi \in \Pi^{\ell}$, then $H^{\pi}=\Lambda^{\pi}$. 
Example 4.27. Recall $\pi$ and $\pi^{\prime}$ from Example 4.26. The sets of possible histories are:

$$
\begin{aligned}
H^{\pi} & =\left\{\left(X, \lambda^{1}\right),\left(X, \lambda^{2}\right),\left(X, \lambda^{3}\right),\left(X, \lambda^{4}\right),\left(Y, \lambda^{1}\right),\left(Y, \lambda^{2}\right),\left(Y, \lambda^{3}\right),\left(Y, \lambda^{4}\right)\right\} \\
H^{\pi^{\prime}} & =\left\{\left(X, \lambda^{1}\right),\left(X, \lambda^{4}\right),\left(Y, \lambda^{2}\right),\left(Y, \lambda^{3}\right),\left(Y, \lambda^{4}\right)\right\} .
\end{aligned}
$$

As both communication strategies are language independent, we have $\Lambda^{\pi}=H^{\pi}$ and $\Lambda^{\pi^{\prime}}=H^{\pi^{\prime}}$.

We next introduce the standard notion of an information correspondence.

Definition 4.28. Let $\pi \in \Pi$. The information correspondence $P_{i}^{\pi}: H^{\pi} \rightrightarrows H^{\pi}$ of $i \in N$ is defined as

$$
P_{i}^{\pi}(\omega, s)=\left\{\left(\omega^{\prime}, s^{\prime}\right) \in H^{\pi} \mid s_{i}^{\prime}=s_{i}\right\}, \quad(\omega, s) \in H^{\pi} .
$$

That is, $P_{i}^{\pi}(\omega, s)$ is the set of histories receiver $i$ considers possible when the true history is $(\omega, s)$. As we call $P_{i}^{\pi}$ an information correspondence, it seems appropriate to briefly show that this name is deserved, i.e., consistent with the common definition of an information correspondence.

Lemma 4.29. Let $\pi \in \Pi$ and $i \in N$. The information correspondence $P_{i}^{\pi}$ satisfies the following two conditions:

C1 For all $(\omega, s) \in H^{\pi},(\omega, s) \in P_{i}^{\pi}(\omega, s)$.

C2 If $\left(\omega^{\prime}, s^{\prime}\right) \in P_{i}^{\pi}(\omega, s)$, then $P_{i}^{\pi}\left(\omega^{\prime}, s^{\prime}\right)=P_{i}^{\pi}(\omega, s)$.

Information correspondences have the property that they partition sets of histories into information sets. In our case we can use $P_{i}^{\pi}$ to define a partition of the set $H^{\pi}$ as

$$
\mathcal{P}_{i}^{\pi}=\left\{P_{i}^{\pi}(\omega, s) \mid(\omega, s) \in H^{\pi}\right\}
$$

This partition reflects $i$ 's knowledge about the true history: whenever the true history is $(\omega, s)$, $i$ knows that the true history lies in $P_{i}^{\pi}(\omega, s)$.

Example 4.30. Recall $\pi$ in Example 4.6. The information correspondence partitions the set of histories as follows:

$$
\begin{aligned}
& P_{1}^{\pi}(X,(v, x))=P_{1}^{\pi}(Y,(v, y))=\{(X,(v, x)),(Y,(v, y))\}, \\
& P_{1}^{\pi}(X,(w, w))=P_{1}^{\pi}(Y,(w, w))=\{(X,(w, w)),(Y,(w, w))\}, \\
& P_{2}^{\pi}(X,(v, x))=\{(X,(v, x)\}, \\
& P_{2}^{\pi}(Y,(v, y))=\{(Y,(v, y))\}, \\
& P_{2}^{\pi}(X,(w, w))=P_{2}^{\pi}(Y,(w, w))=\{(X,(w, w)),(Y,(w, w))\} .
\end{aligned}
$$


Now consider $\pi^{\prime}$ in Example 4.8. The information correspondence partitions the set of histories as follows:

$$
\begin{aligned}
P_{1}^{\pi^{\prime}}(X,(w, x)) & =P_{1}^{\pi^{\prime}}(Y,(w, y))=P_{1}^{\pi^{\prime}}(X,(w, w))=P_{1}^{\pi^{\prime}}(Y,(w, w)) \\
& =\{(X,(w, x)),(Y,(w, y)),(X,(w, w)),(Y,(w, w))\}, \\
P_{2}^{\pi^{\prime}}(X,(w, x)) & =\{(X,(w, x)\}, \\
P_{2}^{\pi^{\prime}}(Y,(w, y)) & =\{(Y,(w, y))\}, \\
P_{2}^{\pi^{\prime}}(X,(w, w))=P_{2}^{\pi^{\prime}}(Y,(w, w)) & =\{(X,(w, w)),(Y,(w, w))\} .
\end{aligned}
$$

It is easy to verify that both $C 1$ and $C 2$ are satisfied. In particular, the information partitions of $\mathcal{P}_{i}^{\pi}$ and, respectively, $\mathcal{P}_{i}^{\pi^{\prime}}$ are given by

$$
\begin{aligned}
& \mathcal{P}_{1}^{\pi}=\{\{(X,(v, x)),(Y,(v, y))\},\{(X,(w, w)),(Y,(w, w))\}\}, \\
& \mathcal{P}_{2}^{\pi}=\{\{(X,(v, x))\},\{(Y,(v, y))\},\{(X,(w, w)),(Y,(w, w))\}\}, \\
& \mathcal{P}_{1}^{\pi^{\prime}}=\{\{(X,(w, x)),(Y,(w, y)),(X,(w, w)),(Y,(w, w))\}\}, \\
& \mathcal{P}_{2}^{\pi^{\prime}}=\{\{(X,(w, x))\},\{(Y,(w, y))\},\{(X,(w, w)),(Y,(w, w))\}\} .
\end{aligned}
$$

Even though $\pi$ and $\pi^{\prime}$ in Example 4.30 induce the same distribution, it is not possible to compare their information partitions since they employ different messages and thus have distinct sets of histories. Still, we can compare such communication strategies via the sets of possible posterior histories of receivers.

Definition 4.31. Let $\pi \in \Pi$. The posterior correspondence $Q_{i}^{\pi}: H^{\pi} \rightrightarrows \Lambda^{\pi}$ of $i \in N$ is defined as

$$
Q_{i}^{\pi}(\omega, s)=\left\{\left(\omega^{\prime}, \lambda^{s^{\prime}}\right) \in \Lambda^{\pi} \mid\left(\omega^{\prime}, s^{\prime}\right) \in P_{i}^{\pi}(\omega, s)\right\}, \quad(\omega, s) \in H^{\pi} .
$$

The set $Q_{i}^{\pi}(\omega, s)$ contains all posterior histories $i$ deems possible if the true history is $(\omega, s)$.

Example 4.32. Recall the information correspondences in Example 4.30. The posterior correspondences related to $\pi$ are as follows.

$$
\begin{aligned}
Q_{1}^{\pi}(X,(t, x)) & =\left\{\left(X,\left(\frac{1}{2}, 1\right)\right),\left(Y,\left(\frac{1}{2}, 0\right)\right)\right\}, \\
Q_{1}^{\pi}(X,(w, w)) & =\left\{\left(X,\left(\frac{1}{2}, \frac{1}{2}\right)\right),\left(Y,\left(\frac{1}{2}, \frac{1}{2}\right)\right)\right\}, \\
Q_{2}^{\pi}(X,(t, x)) & =\left\{\left(X,\left(\frac{1}{2}, 1\right)\right)\right\}, \\
Q_{2}^{\pi}(Y,(t, y)) & =\left\{\left(Y,\left(\frac{1}{2}, 0\right)\right)\right\}, \\
Q_{2}^{\pi}(X,(w, w)) & =\left\{\left(X,\left(\frac{1}{2}, \frac{1}{2}\right)\right),\left(Y,\left(\frac{1}{2}, \frac{1}{2}\right)\right)\right\} .
\end{aligned}
$$


The posterior correspondences related to $\pi^{\prime}$ are as follows.

$$
\begin{aligned}
Q_{1}^{\pi^{\prime}}(X,(w, x)) & =\left\{\left(X,\left(\frac{1}{2}, 1\right)\right),\left(Y,\left(\frac{1}{2}, 0\right)\right),\left(X,\left(\frac{1}{2}, \frac{1}{2}\right)\right),\left(Y,\left(\frac{1}{2}, \frac{1}{2}\right)\right)\right\}, \\
Q_{2}^{\pi^{\prime}}(X,(w, x)) & =\left\{\left(X,\left(\frac{1}{2}, 1\right)\right)\right\}, \\
Q_{2}^{\pi^{\prime}}(Y,(w, y)) & =\left\{\left(Y,\left(\frac{1}{2}, 0\right)\right)\right\}, \\
Q_{2}^{\pi^{\prime}}(X,(w, w)) & =\left\{\left(X,\left(\frac{1}{2}, \frac{1}{2}\right)\right),\left(Y,\left(\frac{1}{2}, \frac{1}{2}\right)\right)\right\} .
\end{aligned}
$$

One can easily see that there is a bijection between the set of histories and the set of posterior histories for both $\pi$ and $\pi^{\prime}$.

For $\pi \in \Pi$ and $i \in N$, define $\mathcal{Q}_{i}^{\pi}=\left\{Q_{i}^{\pi}(\omega, s) \mid(\omega, s) \in H^{\pi}\right\}$. Note that in Example 4.32 both $\mathcal{Q}_{i}^{\pi}$ and $\mathcal{Q}_{i}^{\pi^{\prime}}$ are partitions for any $i \in N$. However, this is not always true.

Example 4.33. Let $N=\{1,2\}, \Omega=\{X, Y\}$, and $\lambda^{0}(X)=1 / 3$. Let communication strategy $\pi \in \Pi$ be given as follows:

\begin{tabular}{c|cccccccc}
$\pi$ & $(x, x)$ & $(x, y)$ & $(y, x)$ & $(y, y)$ & $(a, a)$ & $(a, b)$ & $(b, a)$ & $(b, b)$ \\
\hline$X$ & $\frac{1}{6}$ & 0 & 0 & $\frac{1}{6}$ & $\frac{1}{6}$ & $\frac{1}{6}$ & $\frac{1}{6}$ & $\frac{1}{6}$ \\
$Y$ & 0 & $\frac{1}{12}$ & $\frac{1}{12}$ & $\frac{1}{6}$ & $\frac{1}{12}$ & $\frac{1}{12}$ & $\frac{1}{12}$ & $\frac{5}{12}$
\end{tabular}

For the posterior correspondence we find

$$
\begin{aligned}
& Q_{1}^{\pi}(X,(x, x))=\left\{\left(X,\left(\frac{1}{2}, \frac{1}{2}\right)\right),\left(Y,\left(\frac{1}{2}, \frac{1}{4}\right)\right)\right\}, \\
& Q_{1}^{\pi}(X,(a, a))=\left\{\left(X,\left(\frac{1}{2}, \frac{1}{2}\right)\right),\left(X,\left(\frac{1}{2}, \frac{1}{4}\right)\right),\left(Y,\left(\frac{1}{2}, \frac{1}{2}\right)\right),\left(Y,\left(\frac{1}{2}, \frac{1}{4}\right)\right)\right\} .
\end{aligned}
$$

Since $Q_{1}^{\pi}(X,(x, x)) \neq Q_{1}^{\pi}(X,(a, a))$ and $(X,(1 / 2,1 / 2)) \in Q_{1}^{\pi}(X,(x, x)) \cap Q_{1}^{\pi}(X,(a, a)), \mathcal{Q}_{1}^{\pi}$ is not a partition.

The reason why $Q_{1}^{\pi}$ in Example 4.33 is not a partition is that signals $(x, x)$ and $(a, a)$ lead to the same posterior belief vector, yet $(x, x)$ realizes only in state $X$ whereas $(a, a)$ realizes in both states. This situation, of course, can happen only as long as the communication strategy is not minimal. Thus, $\pi \in \Pi^{\mathrm{m}}$ is sufficient for $\mathcal{Q}_{i}^{\pi}$ to be a partition for all $i \in N$. In order to prove this we define, for $\pi \in \Pi$, the function $\phi: H^{\pi} \rightarrow \Lambda^{\pi}$ by

$$
\phi(\omega, s)=\left(\omega, \lambda^{s}\right), \quad(\omega, s) \in H^{\pi} .
$$

Proposition 4.34. Let $\pi \in \Pi^{\mathrm{m}}$. Then $\phi$ is a bijection and, for every $(\omega, s),\left(\omega^{\prime}, s^{\prime}\right) \in H^{\pi}$ and every $i \in N$, it holds that $(\omega, s) \in P_{i}^{\pi}\left(\omega^{\prime}, s^{\prime}\right)$ if and only if $\phi(\omega, s) \in Q_{i}^{\pi}\left(\omega^{\prime}, s^{\prime}\right)$. In particular, $\mathcal{Q}_{i}^{\pi}$ is a partition.

The converse of Proposition 4.34 is not true. That is, even if the map $\phi$ in (4.9) is a bijection with the required properties, it is still possible that $\pi$ is not minimal. 
Example 4.35. Let $N=\{1,2\}, \Omega=\{X, Y\}$, and $\lambda^{0}(X)=1 / 3$. Let the communication strategy $\pi \in \Pi$ be defined by

\begin{tabular}{c|ccccccc}
$\pi$ & $(a, a)$ & $(b, b)$ & $(a, c)$ & $(c, a)$ & $(b, d)$ & $(d, b)$ & $(e, e)$ \\
\hline$X$ & $\frac{1}{6}$ & 0 & 0 & 0 & $\frac{1}{4}$ & $\frac{1}{6}$ & $\frac{5}{12}$ \\
$Y$ & 0 & $\frac{1}{4}$ & $\frac{1}{6}$ & $\frac{1}{4}$ & 0 & 0 & $\frac{1}{3}$
\end{tabular}

Then, for receiver 1 we have $\lambda_{1}^{(a, a)}(X)=\lambda_{1}^{(b, b)}(X)=1 / 3, \lambda_{1}^{(c, a)}(X)=0, \lambda_{1}^{(d, b)}(X)=1$, and $\lambda_{1}^{(e, e)}(X)=5 / 13$. For receiver 2 we have $\lambda_{2}^{(a, a)}(X)=\lambda_{2}^{(b, b)}(X)=1 / 4, \lambda_{2}^{(a, c)}(X)=0$, $\lambda_{2}^{(b, d)}(X)=1$, and $\lambda_{2}^{(e, e)}(X)=5 / 13$. Note that signals $(a, a)$ and $(b, b)$ lead to the same posterior belief vector, $(1 / 3,1 / 4)$. Thus, $\pi$ is not minimal. For the support of the induced distribution $\sigma$ we find

$$
R(\sigma)=\left\{\left(\frac{1}{3}, \frac{1}{4}\right),\left(\frac{1}{3}, 0\right),\left(0, \frac{1}{4}\right),\left(\frac{1}{3}, 1\right),\left(1, \frac{1}{4}\right),\left(\frac{5}{13}, \frac{5}{13}\right)\right\}
$$

The sets $\mathcal{P}_{1}^{\pi}$ and $\mathcal{Q}_{1}^{\pi}$ defined by the information and posterior correspondences of receiver 1 are as follows:

$$
\begin{aligned}
& \mathcal{P}_{1}^{\pi}=\{(X,(a, a)),(Y,(a, c))\},\{(Y,(c, a))\},\{(X,(b, d)),(Y,(b, b))\},\{(X,(d, b))\}, \\
&\{(X,(e, e)),(Y,(e, e))\}\}, \\
& \mathcal{Q}_{1}^{\pi}=\left\{\left\{\left(X,\left(\frac{1}{3}, \frac{1}{4}\right)\right),\left(Y,\left(\frac{1}{3}, 0\right)\right)\right\},\left\{\left(Y,\left(0, \frac{1}{4}\right)\right)\right\},\left\{\left(X,\left(\frac{1}{3}, 1\right)\right),\left(Y,\left(\frac{1}{3}, \frac{1}{4}\right)\right)\right\},\left\{\left(X,\left(1, \frac{1}{4}\right)\right)\right\},\right. \\
&\left.\left\{\left(X,\left(\frac{5}{13}, \frac{5}{13}\right)\right),\left(Y,\left(\frac{5}{13}, \frac{5}{13}\right)\right)\right\}\right\} .
\end{aligned}
$$

Similar calculations can be made for receiver 2. It is easily checked that not only are $\mathcal{Q}_{1}^{\pi}$ and $\mathcal{Q}_{2}^{\pi}$ partitions, but $\phi$ is a bijection as well. The reason $\mathcal{Q}_{1}^{\pi}$ and $\mathcal{Q}_{2}^{\pi}$ are partitions, even though $\pi \notin \Pi^{\mathrm{m}}$, is that the signals which lead to the same posterior, $(a, a)$ and $(b, b)$, never realize in the same state.

Observe that if $\pi \in \Pi^{\ell}$, then $\phi$ is the identity. Hence, the proposition implies that the partitions $\mathcal{P}_{i}^{\pi}$ and $\mathcal{Q}_{i}^{\pi}$ are identical. For all $\pi \in \Pi^{\mathrm{d}}$, let $\pi^{\ell} \in \Pi^{\ell}$ be defined as in (4.6), i.e., $\pi^{\ell}$ denotes the LICS obtained by replacing the messages of $\pi$ by the posteriors they lead to. Then the posterior history partition of $\pi$ is equal to the history partition of $\pi^{\ell}$. Thus, we have the following corollary which is depicted in the diagram in Figure 4.1.

Corollary 4.36. Let $\pi \in \Pi^{\mathrm{d}}$ and $\pi^{\ell} \in \Pi^{\ell}$ be defined as in (4.6). Then, for all $i \in N$, $\mathcal{Q}_{i}^{\pi}=\mathcal{Q}_{i}^{\pi^{\ell}}=\mathcal{P}_{i}^{\pi^{\ell}}$. 


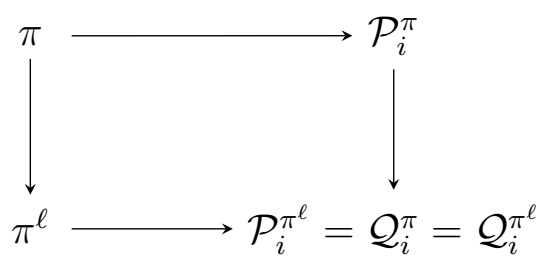

Figure 4.1: Commuting Diagram for $\pi \in \Pi^{\mathrm{d}}$, see Corollary 4.36.

\subsection{Informativeness of Communication Strategies}

Example 4.32 derives the posterior correspondences of the receivers under $\pi$ and $\pi^{\prime}$ from Examples 4.6 and 4.8. Observe that receiver 1 has more precise information about receiver 2's knowledge of the true state under $\pi$ : while he only observes $w$ under $\pi^{\prime}$ and, thus, never learns what message receiver 2 has observed, under $\pi$ upon observing $v$ he knows that receiver 2 knows the true state. In this sense $\pi$ is "more informative": a notion that depends on the posterior correspondence and which we will make more formal soon. Beforehand, we make the brief observation that the posterior correspondence itself is invariant under equivalence or, put differently, that the posterior correspondence is language independent.

Lemma 4.37. Let $\pi, \pi^{\prime} \in \Pi$ with $\pi \sim \pi^{\prime}$. Then, for every $i \in N, \mathcal{Q}_{i}^{\pi}=\mathcal{Q}_{i}^{\pi^{\prime}}$.

We argued in Example 4.32 that the communication strategy $\pi$ is "more informative" for receiver 1 than communication strategy $\pi^{\prime}$. We now give a precise definition of being more informative.

Definition 4.38. Let $\sigma \in \Sigma$ and $\pi, \pi^{\prime} \in \Pi(\sigma)$. The communication strategy $\pi^{\prime}$ is at least as informative as $\pi$ if for all $i \in N$ it holds that

(i) for all $Q^{\prime} \in \mathcal{Q}_{i}^{\pi^{\prime}}$ there exists $Q \in \mathcal{Q}_{i}^{\pi}$ such that $Q^{\prime} \subseteq Q$,

(ii) for all $Q \in \mathcal{Q}_{i}^{\pi}, Q^{\prime} \in \mathcal{Q}_{i}^{\pi^{\prime}}$ with $Q \cap Q^{\prime} \neq \emptyset$ it holds that $Q^{\prime} \subseteq Q$.

Moreover, $\pi$ and $\pi^{\prime}$ are equally informative if $\pi$ is at least as informative as $\pi^{\prime}$ and vice versa; $\pi^{\prime}$ is more informative than $\pi$ if $\pi^{\prime}$ is at least as informative as $\pi$ and not equally informative.

It is easily verified that the notion of being at least as informative is transitive. Our second observation serves as a sanity check: two communication strategies should be equally informative if and only if they induce the same posterior history. And this is true.

Lemma 4.39. Let $\sigma \in \Sigma$ and $\pi, \pi^{\prime} \in \Pi(\sigma)$. Then $\pi$ and $\pi^{\prime}$ are equally informative if and only if $\mathcal{Q}_{i}^{\pi}=\mathcal{Q}_{i}^{\pi^{\prime}}$. 
Two further observations on informativeness are worth mentioning here. First, if $\pi^{\prime}$ is at least as informative as $\pi$, then $\Lambda^{\pi^{\prime}} \subseteq \Lambda^{\pi}$. Second, and an immediate consequence of Lemmas 4.37 and 4.39, equivalent communication strategies are equally informative. This is in line with our interpretation of equivalent communication strategies as using different languages: if the same messages were conveyed in different languages, one would not expect them to become more or less informative.

Example 4.40. Recall the communication strategies $\pi$ and $\pi^{\prime}$ from Examples 4.6 and 4.8. The posterior history correspondences of $\pi$ and $\pi^{\prime}$ were derived in Example 4.32. Note that $\Lambda^{\pi}=\Lambda^{\pi^{\prime}}$ and that $\pi, \pi^{\prime} \in \Pi^{\mathrm{m}}$. Thus, Proposition 4.34 implies that, for every $i \in N, \mathcal{Q}_{i}^{\pi}$ and $\mathcal{Q}_{i}^{\pi^{\prime}}$ are partitions of the same set. More precisely, they are given as

$$
\begin{aligned}
& \mathcal{Q}_{1}^{\pi}=\left\{\left\{\left(X,\left(\frac{1}{2}, 1\right)\right),\left(Y,\left(\frac{1}{2}, 0\right)\right)\right\},\left\{\left(X,\left(\frac{1}{2}, \frac{1}{2}\right)\right),\left(Y,\left(\frac{1}{2}, \frac{1}{2}\right)\right)\right\}\right\}, \\
& \mathcal{Q}_{2}^{\pi}=\left\{\left\{\left(X,\left(\frac{1}{2}, 1\right)\right)\right\},\left\{\left(Y,\left(\frac{1}{2}, 0\right)\right)\right\},\left\{\left(X,\left(\frac{1}{2}, \frac{1}{2}\right)\right),\left(Y,\left(\frac{1}{2}, \frac{1}{2}\right)\right)\right\}\right\}, \\
& \mathcal{Q}_{1}^{\pi^{\prime}}=\left\{\left\{\left(X,\left(\frac{1}{2}, 1\right)\right),\left(Y,\left(\frac{1}{2}, 0\right)\right),\left(X,\left(\frac{1}{2}, \frac{1}{2}\right)\right),\left(Y,\left(\frac{1}{2}, \frac{1}{2}\right)\right)\right\}\right\}, \\
& \mathcal{Q}_{2}^{\pi^{\prime}}=\left\{\left\{\left(X,\left(\frac{1}{2}, 1\right)\right)\right\},\left\{\left(Y,\left(\frac{1}{2}, 0\right)\right)\right\},\left\{\left(X,\left(\frac{1}{2}, \frac{1}{2}\right)\right),\left(Y,\left(\frac{1}{2}, \frac{1}{2}\right)\right)\right\}\right\} .
\end{aligned}
$$

It holds that $\mathcal{Q}_{1}^{\pi}$ is a finer partition than $\mathcal{Q}_{1}^{\pi^{\prime}}$ and that $\mathcal{Q}_{2}^{\pi}=\mathcal{Q}_{2}^{\pi^{\prime}}$. Thus, $\pi$ is more informative than $\pi^{\prime}$.

Note that we do not require $\mathcal{Q}_{i}^{\pi}$ and $\mathcal{Q}_{i}^{\pi^{\prime}}$ to be partitions in order to compare $\pi$ and $\pi^{\prime}$. Nevertheless, if they are partitions, then $\pi^{\prime}$ is more informative than $\pi$ if the restriction of $\mathcal{Q}_{i}^{\pi}$ to $\Lambda^{\pi^{\prime}}$ is coarser than $\mathcal{Q}_{i}^{\pi^{\prime}}$.

Proposition 4.41. Let $\sigma \in \Sigma, \pi, \pi^{\prime} \in \Pi(\sigma)$, and $\Lambda^{\pi^{\prime}} \subseteq \Lambda^{\pi}$. If $\pi \in \Pi^{\mathrm{d}}$, then $\pi^{\prime}$ is at least as informative as $\pi$.

Proposition 4.41 reveals that among those communication strategies that induce the same distribution over posterior belief vectors, those that are direct and have the largest number of posterior histories are the least informative. We can interpret the condition $\Lambda^{\pi^{\prime}} \subseteq \Lambda^{\pi}$ as $\pi^{\prime}$ providing additional information about what posterior histories are impossible. It is worth mentioning that this condition together with the directness of $\pi$ implies that $\mathcal{Q}_{i}^{\pi^{\prime}}$ contains at least the same number of elements as $\mathcal{Q}_{i}^{\pi}$ and that these elements are smaller in the sense of set inclusion.

Consider $\pi, \pi^{\prime} \in \Pi^{\mathrm{d}}$ that satisfy the conditions of Proposition 4.41. In this case $\Lambda^{\pi^{\prime}} \subsetneq \Lambda^{\pi}$ would prevent $\pi$ from being at least as informative as $\pi^{\prime}$. Thus the following corollary is immediate.

Corollary 4.42. Let $\sigma \in \Sigma$ and $\pi, \pi^{\prime} \in \Pi^{\mathrm{d}}(\sigma)$. If $\Lambda^{\pi^{\prime}}=\Lambda^{\pi}$, then $\pi$ and $\pi^{\prime}$ are equally informative. If $\Lambda^{\pi^{\prime}} \subsetneq \Lambda^{\pi}$, then $\pi^{\prime}$ is more informative than $\pi$. 
In Corollary 4.20 a communication strategy is transformed into an LICS that induces the same distribution over posterior vectors. Although they are not equivalent if $\pi$ is not direct, they have the same set of posterior histories as the next lemma shows.

Lemma 4.43. Let $\Delta(\Omega)^{n} \subseteq S$ and $\pi \in \Pi$. For $\pi^{\ell}$ as defined in (4.6) it holds that $\Lambda^{\pi^{\ell}}=\Lambda^{\pi}$.

Proposition 4.41 and Lemma 4.43 immediately imply the following result.

Corollary 4.44. Let $\Delta(\Omega)^{n} \subseteq S, \pi \in \Pi$, and $\pi^{\ell} \in \Pi^{\ell}$ as defined in (4.6). Then $\pi$ is at least as informative as $\pi^{\ell}$.

Corollary 4.44 suggests that using language independent communication strategies reveals as little information as possible. The following example demonstrates that this is, in general, not true.

Example 4.45. Recall $\pi$ and $\pi^{\prime}$ from Example 4.26. Both communication strategies are language independent and, hence, direct. However, as shown in Example 4.27, $\Lambda^{\pi^{\prime}}=H^{\pi^{\prime}} \subsetneq$ $H^{\pi}=\Lambda^{\pi}$. Thus, by Proposition 4.41, $\pi^{\prime}$ is more informative than $\pi$. Observe that it is not relevant that $\pi$ is an LICS: when translating each message sent under $\pi$ in two different languages and sending both with equal probability, we obtain a communication strategy that is not even minimal, but equally informative as $\pi$.

Our final result identifies those communication strategies that are least informative. Let $\sigma \in \Sigma$ and recall that the set $P(\sigma)$ is convex. The relative interior of $P(\sigma)$ is defined as

$$
\operatorname{relint}(P(\sigma))=\left\{p \in P(\sigma) \mid \forall p^{\prime} \in P(\sigma), \exists \alpha>1, \alpha p+(1-\alpha) p^{\prime} \in P(\sigma)\right\}
$$

Proposition 4.46. Let $\Delta(\Omega)^{n} \subseteq S, \sigma \in \Sigma$, and $\pi \in \Pi(\sigma)$. For every $p \in P(\sigma)$, define the communication strategy $\pi^{p} \in \Pi^{\ell}$ by

$$
\pi^{p}(\lambda \mid \omega)=\frac{p(\omega, \lambda)}{\lambda^{0}(\omega)}, \quad \omega \in \Omega, \lambda \in R(\sigma) .
$$

If $p \in \operatorname{relint}(P(\sigma))$, then $\pi$ is at least as informative as $\pi(p)$.

In other words, given a distribution $\sigma \in \Sigma$, if $p$ is in the relative interior of $P(\sigma)$, then $\pi(p)$ is a least informative communication strategy. The proof consists of two steps. First, $\pi$ is at least as informative as the communication strategy $\pi^{\ell}$ that relates to $\pi$ as described in (4.6). It follows from Corollary 4.42 that for any $p^{\prime} \in P(\sigma), \pi\left(p^{\prime}\right)$ is at least as informative as $\pi(p)$, so in particular $\pi^{\ell}$ is at least as informative as $\pi(p)$.

Recall communication strategies $\pi$ and $\pi^{\prime}$ from Example 4.26. We concluded in Example 4.45 that $\pi^{\prime}$ is more informative than $\pi$. The result also follows from Proposition 4.46 since it implies that $\pi$ is a least informative communication strategy as we have $p \in \operatorname{relint}(P(\sigma))$. 


\subsection{Conclusion}

This chapter considers an information design framework with multiple receivers and investigates $(i)$ the inducible distributions of posterior belief vectors and $(i i)$ informativeness of communication strategies. The sender can restrict attention to particular classes of communication strategies without loss of generality. In particular, any distribution over posterior belief vectors can be induced by a language independent communication strategy. Moreover, any direct communication strategy can be transformed into an equivalent LICS.

Extending Kamenica and Gentzkow (2011) by assuming multiple receivers and private communication imposes further constraints on inducible distributions over posterior belief vectors, so that Bayes plausibility is no longer a sufficient condition. We formulate the additional conditions in the form of a linear system of equations that needs to have a non-negative solution. These conditions, together with Bayes plausibility, are necessary and sufficient.

We define informativeness in terms of knowledge about the true posterior history. For every communication strategy there is language independent strategy that is not more informative. Any element in the relative interior of the set of all language independent communication strategies which induce a particular distribution belongs to the set of least informative communication strategies.

\subsection{Appendix}

Proof of Proposition 4.1. Let $\hat{\sigma}=\alpha \sigma+(1-\alpha) \sigma^{\prime}$.

If there is $i \in N$ such that $\left|S_{i}\right|<\left|R\left(\sigma_{i}\right) \cup R\left(\sigma_{i}^{\prime}\right)\right|$, then there are not sufficient messages to implement all of $i$ 's possible beliefs in $R\left(\hat{\sigma}_{i}\right)$.

For the other direction, let $\left|S_{i}\right| \geq\left|R\left(\sigma_{i}\right) \cup R\left(\sigma_{i}^{\prime}\right)\right|$ for all $i \in N$. Let $\pi, \pi^{\prime} \in \Pi$ be such that $\sigma^{\pi}=\sigma$ and $\sigma^{\pi^{\prime}}=\sigma^{\prime}$. Since $\left|S_{i}\right| \geq\left|R\left(\sigma_{i}\right) \cup R\left(\sigma_{i}^{\prime}\right)\right|$, we can assume without loss of generality that there is $s \in S$ with $s_{i} \in S_{i}^{\pi} \cap S_{i}^{\pi^{\prime}}$ if and only if there are $\lambda \in R(\sigma)$ and $\lambda^{\prime} \in R\left(\sigma^{\prime}\right)$ such that $\lambda_{i}=\lambda_{i}^{\prime}=\lambda_{i}^{s}$.

Let $\hat{\pi}=\alpha \pi+(1-\alpha) \pi^{\prime}$. Let $s \in S^{\hat{\pi}}$ and $i \in N$. Without loss of generality let $s_{i} \in S_{i}^{\pi}$. Assume first that $s_{i} \notin S_{i}^{\pi^{\prime}}$. It holds that, for every $\omega \in \Omega$,

$$
\hat{\lambda}_{i}^{s}(\omega)=\frac{\hat{\pi}_{i}\left(s_{i} \mid \omega\right) \lambda^{0}(\omega)}{\sum_{\omega^{\prime} \in \Omega} \hat{\pi}_{i}\left(s_{i} \mid \omega^{\prime}\right) \lambda^{0}\left(\omega^{\prime}\right)}=\frac{\alpha \pi_{i}\left(s_{i} \mid \omega\right) \lambda^{0}(\omega)}{\alpha \sum_{\omega^{\prime} \in \Omega} \pi_{i}\left(s_{i} \mid \omega^{\prime}\right) \lambda^{0}\left(\omega^{\prime}\right)}=\lambda_{i}^{s}(\omega) .
$$

Assume next that $s_{i} \in S_{i}^{\pi^{\prime}}$ and observe that in this case

$$
\frac{\pi_{i}\left(s_{i} \mid \omega\right) \lambda^{0}(\omega)}{\sum_{\omega^{\prime} \in \Omega} \pi_{i}\left(s_{i} \mid \omega^{\prime}\right) \lambda^{0}\left(\omega^{\prime}\right)}=\frac{\pi_{i}^{\prime}\left(s_{i} \mid \omega\right) \lambda^{0}(\omega)}{\sum_{\omega^{\prime} \in \Omega} \pi_{i}^{\prime}\left(s_{i} \mid \omega^{\prime}\right) \lambda^{0}\left(\omega^{\prime}\right)} .
$$

Thus,

$$
\hat{\lambda}_{i}^{s}(\omega)=\frac{\alpha \pi_{i}\left(s_{i} \mid \omega\right) \lambda^{0}(\omega)+(1-\alpha) \pi_{i}^{\prime}\left(s_{i} \mid \omega\right) \lambda^{0}(\omega)}{\alpha \sum_{\omega^{\prime} \in \Omega} \pi_{i}\left(s_{i} \mid \omega^{\prime}\right) \lambda^{0}\left(\omega^{\prime}\right)+(1-\alpha) \sum_{\omega^{\prime} \in \Omega} \pi_{i}^{\prime}\left(s_{i} \mid \omega^{\prime}\right) \lambda^{0}\left(\omega^{\prime}\right)}=\lambda_{i}^{s}(\omega) .
$$


We have shown that $R(\hat{\sigma})=R(\sigma) \cup R\left(\sigma^{\prime}\right)$. We now have, for every $\lambda \in \Delta(\Omega)^{n}$,

$$
\begin{aligned}
\hat{\sigma}(\lambda) & =\sum_{s \in S^{\hat{\pi}}: \hat{\lambda}^{s}=\lambda} \sum_{\omega \in \Omega} \hat{\pi}(s \mid \omega) \lambda^{0}(\omega) \\
& =\alpha \sum_{s \in S^{\pi}: \lambda^{s}=\lambda} \sum_{\omega \in \Omega} \pi(s \mid \omega) \lambda^{0}(\omega)+(1-\alpha) \sum_{s \in S^{\pi^{\prime}}: \lambda^{\prime s}=\lambda} \sum_{\omega \in \Omega} \pi^{\prime}(s \mid \omega) \lambda^{0}(\omega) \\
& =\alpha \sigma(\lambda)+(1-\alpha) \sigma^{\prime}(\lambda) .
\end{aligned}
$$

Hence, $\hat{\pi}$ induces $\hat{\sigma}$.

Proof of Proposition 4.3. Assume $\pi \in \Pi$ is such that $\sigma^{\pi}=\sigma$ with $\sigma(\lambda)>0$. Suppose that $\prod_{i \in N} \lambda_{i}(\omega)=0$ for all $\omega \in \Omega$, that is, for all $\omega \in \Omega$ there exists $i_{\omega} \in N$ such that $\lambda_{i_{\omega}}(\omega)=0$. Let $s \in S^{\pi}$ be such that $\lambda^{s}=\lambda$. Then it holds that, for all $\omega \in \Omega, \pi(s \mid \omega) \leq \pi_{i_{\omega}}\left(s_{i_{\omega}} \mid \omega\right)=0$. We find by (4.2) that $\sigma(\lambda)=0$, leading to a contradiction. Consequently, there exists $\omega \in \Omega$ such that $\prod_{i \in N} \lambda_{i}(\omega)>0$.

For the converse, assume there exists $\omega \in \Omega$ such that $\prod_{i \in N} \lambda_{i}(\omega)>0$. Let $i \in N$ and $\beta_{i}=\max _{\omega \in \Omega}\left(\lambda_{i}(\omega) / \lambda^{0}(\omega)\right)$ be the highest ratio across states of posterior belief to prior belief for receiver $i$. Let $x_{i}, y_{i} \in S_{i}$ be two distinct messages. We define, for every $\omega \in \Omega$,

$$
\begin{array}{rlrl}
\rho_{i}\left(x_{i} \mid \omega\right) & =\frac{1}{\beta_{i}} \frac{\lambda_{i}(\omega)}{\lambda^{0}(\omega)}, & \\
\rho_{i}\left(y_{i} \mid \omega\right) & =1-\rho_{i}\left(x_{i} \mid \omega\right), & \\
\rho_{i}\left(s_{i} \mid \omega\right) & =0, & & s_{i} \in S_{i} \backslash\left\{x_{i}, y_{i}\right\} .
\end{array}
$$

Notice that $\rho_{i}\left(x_{i} \mid \omega\right) \leq 1$. We define $\pi: \Omega \rightarrow \Delta(S)$ by

$$
\pi(s \mid \omega)=\prod_{i \in N} \rho_{i}\left(s_{i} \mid \omega\right), \quad s \in S, \omega \in \Omega
$$

It holds that $\pi$ is a communication strategy with $\pi_{i}\left(s_{i} \mid \omega\right)=\rho_{i}\left(s_{i} \mid \omega\right)$ for every receiver $i \in N$.

Let $i \in N$. For every $s \in S^{\pi}$ with $s_{i}=x_{i}$ it holds that

$$
\lambda_{i}^{s}(\omega)=\frac{\pi_{i}\left(x_{i} \mid \omega\right) \lambda^{0}(\omega)}{\sum_{\omega^{\prime} \in \Omega} \pi_{i}\left(x_{i} \mid \omega^{\prime}\right) \lambda^{0}\left(\omega^{\prime}\right)}=\frac{\frac{1}{\beta_{i}} \frac{\lambda_{i}(\omega)}{\lambda^{0}(\omega)} \lambda^{0}(\omega)}{\frac{1}{\beta_{i}} \sum_{\omega^{\prime} \in \Omega} \frac{\lambda_{i}\left(\omega^{\prime}\right)}{\lambda^{0}\left(\omega^{\prime}\right)} \lambda^{0}\left(\omega^{\prime}\right)}=\frac{\lambda_{i}(\omega)}{\sum_{\omega^{\prime} \in \Omega} \lambda_{i}\left(\omega^{\prime}\right)}=\lambda_{i}(\omega), \quad \omega \in \Omega
$$

We have that $\lambda^{\bar{x}}=\lambda$, where $\bar{x}=\left(x_{1}, \ldots, x_{n}\right)$.

Let $\omega \in \Omega$ be such that $\lambda_{i}(\omega)>0$. Then

$$
\sigma(\lambda) \geq \pi(\bar{x} \mid \omega) \lambda^{0}(\omega)=\lambda^{0}(\omega) \prod_{i \in N} \rho_{i}\left(x_{i} \mid \omega\right)>0
$$

which implies that $\lambda \in R\left(\sigma^{\pi}\right)$. 
Proof of Proposition 4.4. Proposition 4.3 implies necessity. For the other direction, let $R_{i}=\left\{\lambda_{i}^{1}, \ldots, \lambda_{i}^{m_{i}}\right\}$, let $\left\{x_{i}^{1}, \ldots, x_{i}^{m_{i}}, y_{i}\right\} \subseteq S_{i}$ be such that $x_{i}^{k} \neq x_{i}^{\ell}, y_{i}$ for all $k \neq \ell$ and all $i \in N$. Let $R=\left\{\lambda^{1}, \ldots, \lambda^{m}\right\}$ and define $\pi^{1}, \ldots, \pi^{m}$ as in the proof of Proposition 4.3, where, for all $i \in N$ and all $k=1, \ldots, m$ one has $\lambda^{k} \in R\left(\sigma^{\pi^{k}}\right)$ and $S_{i}^{\pi^{k}} \subseteq\left\{x_{i}^{k}, y_{i}\right\}$. Let $\alpha^{1}, \ldots, \alpha^{m}>0$ with $\sum_{k=1}^{m} \alpha^{k}=1$, and let $\sigma=\sum_{k=1}^{m} \alpha^{k} \sigma^{\pi^{k}}$. Since $\left|S_{i}\right| \geq m_{i}+1=\left|\bigcup_{k=1}^{m} R\left(\sigma_{i}^{\pi^{k}}\right)\right|$, iterative application of Proposition 4.1 implies that $\sigma \in \Sigma$. Moreover, by construction, $\sigma^{\pi}\left(\lambda^{k}\right)=$ $\alpha^{k} \sigma^{\pi^{k}}\left(\lambda^{k}\right)>0$.

Proof of Lemma 4.9. Let $\pi \in \Pi^{\mathrm{d}}$. For each $i \in N$ there exists a bijection $\phi_{i}: S_{i}^{\pi} \rightarrow R\left(\sigma_{i}^{\pi}\right)$ since $\pi$ is direct. In particular, for every $s \in S^{\pi}$, we have $\lambda^{s}=\left(\phi_{i}\left(s_{i}\right)\right)_{i \in N}$ so that there is a bijection between $S^{\pi}$ and $R\left(\sigma^{\pi}\right)$. Hence, $\left|S^{\pi}\right|=\left|R\left(\sigma^{\pi}\right)\right|$, that is, $\pi$ is minimal.

Proof of Lemma 4.13. Let $\pi \in \Pi^{\ell}, s \in S^{\pi}$, and $i \in N$. It holds that $\lambda_{i}^{s}=s_{i}$ by definition of an LICS. This defines an identity between $S_{i}^{\pi}$ and $R\left(\sigma_{i}^{\pi}\right)$. It follows that $\left|S_{i}^{\pi}\right|=\left|R\left(\sigma_{i}^{\pi}\right)\right|$.

Proof of Proposition 4.17. For every $i \in N$ there is a bijection $\psi_{i}: S_{i}^{\pi} \rightarrow \hat{S}_{i}^{\hat{\pi}}$ such that, for every $\omega \in \Omega$, for every $s \in S^{\pi}, \hat{\pi}(\psi(s) \mid \omega)=\pi(s \mid \omega)$. Let $s \in S^{\pi}$ and $\hat{s} \in \hat{S}^{\hat{\pi}}$ be such that $\psi(s)=\hat{s}$. It follows from (4.5) that $\lambda^{s}=\hat{\lambda}^{\hat{s}}$. Since $\hat{S}^{\hat{\pi}}=\psi\left(S^{\pi}\right)$, we have that $R\left(\sigma^{\hat{\pi}}\right)=R\left(\sigma^{\pi}\right)$. Moreover, it holds that, for every $\lambda \in R\left(\sigma^{\pi}\right)$,

$$
\begin{aligned}
\sigma^{\pi}(\lambda) & =\sum_{s \in S^{\pi}: \lambda^{s}=\lambda} \sum_{\omega \in \Omega} \pi(s \mid \omega) \lambda^{0}(\omega)=\sum_{s \in S^{\pi}: \lambda^{s}=\lambda} \sum_{\omega \in \Omega} \hat{\pi}(\psi(s) \mid \omega) \lambda^{0}(\omega) \\
& =\sum_{\hat{s} \in \hat{S}^{\hat{\pi}}: \hat{\lambda}^{\hat{s}}=\lambda} \sum_{\omega \in \Omega} \hat{\pi}(\hat{s} \mid \omega) \lambda^{0}(\omega)=\sigma^{\hat{\pi}}(\lambda) .
\end{aligned}
$$

Proof of Proposition 4.18. By Proposition 4.17 it holds that $\sigma^{\pi}=\sigma^{\pi^{\prime}}$, so $S^{\pi}=R\left(\sigma^{\pi}\right)=$ $R\left(\sigma^{\pi^{\prime}}\right)=S^{\pi^{\prime}}$. As $\pi \sim \pi^{\prime}$, for every $i \in N$ there is a bijection $\psi_{i}: S_{i}^{\pi} \rightarrow S_{i}^{\pi^{\prime}}$ such that, for every $\omega \in \Omega$, for every $s \in S^{\pi}, \pi^{\prime}(\psi(s) \mid \omega)=\pi(s \mid \omega)$. In particular, since $\pi, \pi^{\prime} \in \Pi^{\ell}$, we have, for every $i \in N$, for every $\lambda \in S^{\pi}$,

$$
\psi_{i}\left(\lambda_{i}\right)(\omega)=\frac{\pi_{i}^{\prime}\left(\psi_{i}\left(\lambda_{i}\right) \mid \omega\right) \lambda^{0}(\omega)}{\sum_{\omega^{\prime} \in \Omega} \pi_{i}^{\prime}\left(\psi_{i}\left(\lambda_{i}\right) \mid \omega^{\prime}\right) \lambda^{0}\left(\omega^{\prime}\right)}=\frac{\pi_{i}\left(\lambda_{i} \mid \omega\right) \lambda^{0}(\omega)}{\sum_{\omega^{\prime} \in \Omega} \pi_{i}\left(\lambda_{i} \mid \omega^{\prime}\right) \lambda^{0}\left(\omega^{\prime}\right)}=\lambda_{i}(\omega), \quad \omega \in \Omega,
$$

where the first and third equality follow since $\pi, \pi^{\prime} \in \Pi^{\ell}$, and the second equality uses (4.5). It follows that $\pi=\pi^{\prime}$.

Proof of Theorem 4.22. Assume that $\sigma$ is Bayes plausible and there exists $p \in \mathbb{R}_{+}^{\Omega \times R(\sigma)}$ such that $(i)$ and $(i i)$ are satisfied. Let $\pi$ be defined as in (4.7). We first show that $\pi$ is a communication strategy. 
Let $\omega \in \Omega$. Obviously, it holds that, for every $\lambda \in \Delta(\Omega)^{n}, \pi(\lambda \mid \omega) \geq 0$. In formula (4.11) that follows next, $i \in N$ is an arbitrarily chosen receiver. It holds that

$$
\sum_{\lambda \in S^{\pi}} p(\omega, \lambda)=\sum_{\lambda_{i} \in R_{i}\left(\sigma_{i}\right)} \sum_{\lambda^{\prime} \in R(\sigma): \lambda_{i}^{\prime}=\lambda_{i}} p\left(\omega, \lambda^{\prime}\right) \stackrel{(i i)}{=} \sum_{\lambda_{i} \in R_{i}\left(\sigma_{i}\right)} \lambda_{i}(\omega) \sigma_{i}\left(\lambda_{i}\right)=\lambda^{0}(\omega),
$$

where the last equality is true as $\sigma$ is Bayes plausible. We find that

$$
\sum_{\lambda \in S^{\pi}} \pi(\lambda \mid \omega)=\sum_{\lambda \in S^{\pi}} \frac{p(\omega, \lambda)}{\lambda^{0}(\omega)} \stackrel{(4.11)}{=} \frac{\lambda^{0}(\omega)}{\lambda^{0}(\omega)}=1,
$$

which proves that $\pi$ is a communication strategy.

Next, we show that $\pi$ is an LICS. Let $\omega \in \Omega, i \in N$, and $\lambda_{i} \in R\left(\sigma_{i}\right)$. It holds that

$$
\begin{aligned}
\frac{\pi_{i}\left(\lambda_{i} \mid \omega\right) \lambda^{0}(\omega)}{\sum_{\omega^{\prime} \in \Omega} \pi_{i}\left(\lambda_{i} \mid \omega^{\prime}\right) \lambda^{0}\left(\omega^{\prime}\right)} & =\frac{\sum_{\lambda^{\prime} \in R(\sigma): \lambda_{i}^{\prime}=\lambda_{i}} \pi\left(\lambda^{\prime} \mid \omega\right) \lambda^{0}(\omega)}{\sum_{\omega^{\prime} \in \Omega} \sum_{\lambda^{\prime} \in R(\sigma): \lambda_{i}^{\prime}=\lambda_{i}} \pi\left(\lambda^{\prime} \mid \omega^{\prime}\right) \lambda^{0}\left(\omega^{\prime}\right)} \\
& \stackrel{(4.7)}{=} \frac{\sum_{\lambda^{\prime} \in R(\sigma): \lambda_{i}^{\prime}=\lambda_{i}} \frac{p\left(\omega, \lambda^{\prime}\right)}{\lambda^{0}(\omega)} \lambda^{0}(\omega)}{\sum_{\omega^{\prime} \in \Omega} \sum_{\lambda^{\prime} \in R(\sigma): \lambda_{i}^{\prime}=\lambda_{i}} \frac{p\left(\omega^{\prime}, \lambda^{\prime}\right)}{\lambda^{0}\left(\omega^{\prime}\right)} \lambda^{0}\left(\omega^{\prime}\right)} \\
& =\frac{\sum_{\lambda^{\prime} \in R(\sigma): \lambda_{i}^{\prime}=\lambda_{i}} p\left(\omega, \lambda^{\prime}\right)}{\sum_{\omega^{\prime} \in \Omega} \sum_{\lambda^{\prime} \in R(\sigma): \lambda_{i}^{\prime}=\lambda_{i}} p\left(\omega^{\prime}, \lambda^{\prime}\right)} \\
& \stackrel{(i i)}{=} \frac{\lambda_{i}(\omega) \sigma_{i}\left(\lambda_{i}\right)}{\sum_{\lambda^{\prime} \in R(\sigma): \lambda_{i}^{\prime}=\lambda_{i}} \sum_{\omega^{\prime} \in \Omega} p\left(\omega^{\prime}, \lambda^{\prime}\right)} \\
& \stackrel{(i)}{=} \frac{\lambda_{i}(\omega) \sigma_{i}\left(\lambda_{i}\right)}{\sum_{\lambda^{\prime} \in R(\sigma): \lambda_{i}^{\prime}=\lambda_{i}} \sigma\left(\lambda^{\prime}\right)} \\
& =\frac{\lambda_{i}(\omega) \sigma_{i}\left(\lambda_{i}\right)}{\sigma_{i}\left(\lambda_{i}\right)} \\
& =\lambda_{i}(\omega) .
\end{aligned}
$$

As message $\lambda_{i}$ leads to posterior $\lambda_{i}, \pi$ is an LICS.

We show next that $\sigma^{\pi}=\sigma$. Let $\lambda \in R(\sigma)$. It holds that

$$
\sigma^{\pi}(\lambda)=\sum_{\omega \in \Omega} \pi(\lambda \mid \omega) \lambda^{0}(\omega)=\sum_{\omega \in \Omega} \frac{p(\omega, \lambda)}{\lambda^{0}(\omega)} \lambda^{0}(\omega)=\sum_{\omega \in \Omega} p(\omega, \lambda) \stackrel{(i)}{=} \sigma(\lambda) .
$$

At this point we have shown that $\sigma$ is inducible if $R\left(\sigma_{i}\right) \subseteq S_{i}$. Recall that $\left|S_{i}\right| \geq R\left(\sigma_{i}\right)$. For every $i \in N$, let $T_{i}$ be a subset of $S_{i}$ with cardinality equal to $\left|R\left(\sigma_{i}\right)\right|$ and take a bijection $\psi_{i}: R\left(\sigma_{i}\right) \rightarrow T_{i}$. Define the communication strategy $\pi^{\prime}: \Omega \rightarrow \Delta(S)$ by

$$
\pi^{\prime}(\psi(\lambda) \mid \omega)=\pi(\lambda \mid \omega), \quad \omega \in \Omega, \lambda \in R(\sigma) .
$$

Then $\pi \sim \pi^{\prime}$, so by Proposition 4.17 we have that $\sigma^{\pi^{\prime}}=\sigma^{\pi}=\sigma$. It follows that $\sigma \in \Sigma$.

Now assume that $\sigma \in \Sigma$. It follows from Proposition 4.2 that $\sigma$ is Bayes plausible. Let $\pi \in \Pi$ be such that $\sigma^{\pi}=\sigma$. For every $\omega \in \Omega$, for every $\lambda \in R(\sigma)$, define

$$
p(\omega, \lambda)=\sum_{s \in S^{\pi}: \lambda^{s}=\lambda} \pi(s \mid \omega) \lambda^{0}(\omega) .
$$


We first show that $(i)$ holds. We have that

$$
\sigma(\lambda)=\sum_{s \in S^{\pi}: \lambda^{s}=\lambda} \sum_{\omega \in \Omega} \pi(s \mid \omega) \lambda^{0}(\omega) \stackrel{(4.12)}{=} \sum_{\omega \in \Omega} p(\omega, \lambda), \quad \lambda \in R(\sigma) .
$$

Next, we show (ii) holds. Let $\omega \in \Omega, i \in N$, and $\lambda_{i} \in R\left(\sigma_{i}\right)$. We have that

$$
\begin{aligned}
\lambda_{i}(\omega) \sigma_{i}\left(\lambda_{i}\right) & =\frac{\sum_{s_{i} \in S_{i}^{\pi}: \lambda_{i}^{s}=\lambda_{i}} \pi_{i}\left(s_{i} \mid \omega\right) \lambda^{0}(\omega)}{\sum_{\omega^{\prime} \in \Omega} \sum_{s_{i} \in S_{i}^{\pi}: \lambda_{i}^{s}=\lambda_{i}} \pi_{i}\left(s_{i} \mid \omega^{\prime}\right) \lambda^{0}\left(\omega^{\prime}\right)} \sum_{\lambda^{\prime} \in R(\sigma): \lambda_{i}^{\prime}=\lambda_{i}} \sigma\left(\lambda^{\prime}\right) \\
& =\frac{\sum_{s_{i} \in S_{i}^{\pi}: \lambda_{i}^{s}=\lambda_{i}} \pi_{i}\left(s_{i} \mid \omega\right) \lambda^{0}(\omega)}{\sum_{\omega^{\prime} \in \Omega} \sum_{s_{i} \in S_{i}^{\pi}: \lambda_{i}^{s}=\lambda_{i}} \pi_{i}\left(s_{i} \mid \omega^{\prime}\right) \lambda^{0}\left(\omega^{\prime}\right)} \sum_{\lambda^{\prime} \in R(\sigma): \lambda_{i}^{\prime}=\lambda_{i}} \sum_{s \in S^{\pi}: \lambda^{s}=\lambda^{\prime}} \sum_{\omega^{\prime} \in \Omega} \pi\left(s \mid \omega^{\prime}\right) \lambda^{0}\left(\omega^{\prime}\right) \\
& =\frac{\sum_{s_{i} \in S_{i}^{\pi}: \lambda_{i}^{s}=\lambda_{i}} \pi_{i}\left(s_{i} \mid \omega\right) \lambda^{0}(\omega)}{\sum_{\omega^{\prime} \in \Omega} \sum_{s_{i} \in S_{i}^{\pi}: \lambda_{i}^{s}=\lambda_{i}} \pi_{i}\left(s_{i} \mid \omega^{\prime}\right) \lambda^{0}\left(\omega^{\prime}\right)} \sum_{\omega^{\prime} \in \Omega} \sum_{s_{i} \in S_{i}^{\pi}: \lambda_{i}^{s}=\lambda_{i}} \pi_{i}\left(s_{i} \mid \omega^{\prime}\right) \lambda^{0}\left(\omega^{\prime}\right) \\
& =\sum_{s_{i} \in S_{i}^{\pi}: \lambda_{i}^{s}=\lambda_{i}} \pi_{i}\left(s_{i} \mid \omega\right) \lambda^{0}(\omega) \\
& =\sum_{\lambda^{\prime} \in R(\sigma): \lambda_{i}^{\prime}=\lambda_{i} s \in S^{\pi}: \lambda^{s}=\lambda^{\prime}} \pi(s \mid \omega) \lambda^{0}(\omega) \\
& =\sum_{\lambda^{\prime} \in R(\sigma): \lambda_{i}^{\prime}=\lambda_{i}} p\left(\omega, \lambda^{\prime}\right),
\end{aligned}
$$

where the first equality follows from Lemma 4.19.

We are now ready to provide the proofs of Theorem 4.10 and Proposition 4.15.

Proof of Theorem 4.10. Let $\sigma \in \Sigma$. Then it holds that, for every $i \in N,\left|S_{i}\right| \geq R\left(\sigma_{i}\right)$. Theorem 4.22 implies that there is an LICS $\pi: \Omega \rightarrow \Delta\left(\Delta(\Omega)^{n}\right)$ which induces $\sigma$. For every $i \in N$, let $T_{i}$ be a subset of $S_{i}$ with cardinality equal to $\left|R\left(\sigma_{i}\right)\right|$ and take a bijection $\psi_{i}$ : $R\left(\sigma_{i}\right) \rightarrow T_{i}$. Let the communication strategy $\pi^{\prime}: \Omega \rightarrow \Delta(S)$ be defined by

$$
\pi^{\prime}(\psi(\lambda) \mid \omega)=\pi(\lambda \mid \omega), \quad \omega \in \Omega, \lambda \in R(\sigma)
$$

Then $\pi \sim \pi^{\prime}$, so by Proposition 4.17 we have that $\sigma^{\pi^{\prime}}=\sigma^{\pi}=\sigma$. As the LICS $\pi$ is direct, it follows that $\pi^{\prime} \in \Pi^{\mathrm{d}}$.

Proof of Proposition 4.15. As $P(\sigma)$ is a non-empty, compact, and convex polytope by Corollary 4.23 and $\Pi^{\ell}(\sigma)$ is a linear transformation of $P(\sigma)$ by $(4.7), \Pi^{\ell}(\sigma)$ is a non-empty, compact, and convex polytope as well.

Proof of Proposition 4.25. Assume that there is $p \in \mathbb{R}_{+}^{\Omega \times R}$ such $(i)$ and $(i i)$ hold. Let $\pi: \Omega \rightarrow \Delta(R)$ be as defined in (4.8). We have that

$$
\sum_{\lambda^{\prime} \in R} \pi\left(\lambda^{\prime} \mid \omega\right) \stackrel{(4.8)}{=} \sum_{\lambda^{\prime} \in R} \frac{p\left(\omega, \lambda^{\prime}\right)}{\lambda^{0}(\omega)} \stackrel{(i)}{=} \frac{\lambda^{0}(\omega)}{\lambda^{0}(\omega)}=1, \quad \omega \in \Omega .
$$


Moreover, for every $\omega \in \Omega, i \in N$, and $\lambda_{i} \in S_{i}^{\pi}$, it holds that

$$
\begin{aligned}
\frac{\sum_{\lambda^{\prime} \in R: \lambda_{i}^{\prime}=\lambda_{i}} \pi\left(\lambda^{\prime} \mid \omega\right) \lambda^{0}(\omega)}{\sum_{\omega^{\prime} \in \Omega} \sum_{\lambda^{\prime} \in R: \lambda_{i}^{\prime}=\lambda_{i}} \pi\left(\lambda^{\prime} \mid \omega^{\prime}\right) \lambda^{0}\left(\omega^{\prime}\right)} \stackrel{\stackrel{(4.8)}{=} \frac{\sum_{\lambda^{\prime} \in R: \lambda_{i}^{\prime}=\lambda_{i}} p\left(\omega, \lambda^{\prime}\right)}{\sum_{\omega^{\prime} \in \Omega} \sum_{\lambda^{\prime} \in R: \lambda_{i}^{\prime}=\lambda_{i}} p\left(\omega^{\prime}, \lambda^{\prime}\right)}}{ } \\
\stackrel{(i i)}{=} \frac{\lambda_{i}(\omega) \sum_{\omega^{\prime} \in \Omega} \sum_{\lambda^{\prime} \in R: \lambda_{i}^{\prime}=\lambda_{i}} p\left(\omega^{\prime}, \lambda^{\prime}\right)}{\sum_{\omega^{\prime} \in \Omega} \sum_{\lambda^{\prime} \in R: \lambda_{i}^{\prime}=\lambda_{i}} p\left(\omega^{\prime}, \lambda^{\prime}\right)}=\lambda_{i}(\omega) .
\end{aligned}
$$

Thus, $\pi$ is an LICS and $R\left(\sigma^{\pi}\right)=S^{\pi} \subseteq R$.

In order to account for message sets $S_{i}$ that do not allow for language independent messages, note that, for all $i \in N,\left|R_{i}\left(\sigma_{i}^{\pi}\right)\right| \leq\left|R_{i}\right| \leq\left|S_{i}\right|$. For every $i \in N$ let $T_{i}$ be a subset of $S_{i}$ with $\left|T_{i}\right|=\left|R_{i}\left(\sigma^{\pi_{i}}\right)\right|$ and take a bijection $\psi_{i}: R_{i}\left(\sigma_{i}^{\pi}\right) \rightarrow T_{i}$. Let the communication strategy $\pi^{\prime}: \Omega \rightarrow \Delta(S)$ be defined by

$$
\pi^{\prime}(\psi(\lambda) \mid \omega)=\pi(\lambda \mid \omega), \quad \omega \in \Omega, \lambda \in R\left(\sigma^{\pi}\right) .
$$

It holds that $\pi \sim \pi^{\prime}$, so by Proposition 4.17 we have that $\sigma^{\pi^{\prime}}=\sigma^{\pi}$ and $R\left(\sigma^{\pi^{\prime}}\right)=R\left(\sigma^{\pi}\right) \subseteq R$.

Now assume that $\sigma \in \Sigma$ is such that $R(\sigma) \subseteq R$. Then, by Theorem 4.22, there is an LICS $\pi: \Omega \rightarrow \Delta(R)$ that induces $\sigma$. Let

$$
p(\omega, \lambda)=\pi(\lambda \mid \omega) \lambda^{0}(\omega), \quad \omega \in \Omega, \lambda \in R
$$

By construction, $S^{\pi}=R(\sigma) \subseteq R$ and $p(\omega, \lambda)=0$ for all $\lambda \in R \backslash S^{\pi}$ and all $\omega \in \Omega$. So, $(i)$ is satisfied since

$$
\sum_{\lambda^{\prime} \in R} p\left(\omega, \lambda^{\prime}\right) \stackrel{(4.13)}{=} \sum_{\lambda^{\prime} \in R} \pi\left(\lambda^{\prime} \mid \omega\right) \lambda^{0}(\omega)=\lambda^{0}(\omega) \sum_{\lambda^{\prime} \in S^{\pi}} \pi\left(\lambda^{\prime} \mid \omega\right)=\lambda^{0}(\omega), \quad \omega \in \Omega .
$$

Further, for every $\omega \in \Omega, i \in N$, and $\lambda_{i} \in R_{i}$, it holds that

$$
\begin{aligned}
\sum_{\lambda^{\prime} \in R: \lambda_{i}^{\prime}=\lambda_{i}} p\left(\omega, \lambda^{\prime}\right) & \stackrel{(4.13)}{=} \sum_{\lambda^{\prime} \in R: \lambda_{i}^{\prime}=\lambda_{i}} \pi\left(\lambda^{\prime} \mid \omega\right) \lambda^{0}(\omega)=\pi_{i}\left(\lambda_{i} \mid \omega\right) \lambda^{0}(\omega) \\
& \stackrel{(4.1)}{=} \lambda_{i}(\omega) \sum_{\omega^{\prime} \in \Omega} \pi_{i}\left(\lambda_{i} \mid \omega^{\prime}\right) \lambda^{0}\left(\omega^{\prime}\right)=\lambda_{i}(\omega) \sum_{\omega^{\prime} \in \Omega} \sum_{\lambda^{\prime} \in R: \lambda_{i}^{\prime}=\lambda_{i}} \pi\left(\lambda^{\prime} \mid \omega^{\prime}\right) \lambda^{0}\left(\omega^{\prime}\right) \\
& \stackrel{(4.13)}{=} \lambda_{i}(\omega) \sum_{\omega^{\prime} \in \Omega} \sum_{\lambda^{\prime} \in R: \lambda_{i}^{\prime}=\lambda_{i}} p\left(\omega^{\prime}, \lambda^{\prime}\right) .
\end{aligned}
$$

Hence, (ii) is satisfied.

Lastly, let $p$ be such that, for all $\lambda \in R, \sum_{\omega \in \Omega} p(\lambda, \omega)>0$. Then for each $\lambda \in R$, there is $\omega \in \Omega$ such that $\pi(\lambda \mid \omega)>0$. Thus, $R\left(\sigma^{\pi}\right)=S^{\pi}=R$.

Proof of Lemma 4.29. Let $(\omega, s) \in H^{\pi}$. Suppose $(\omega, s) \notin P_{i}^{\pi}(\omega, s)$. Then, $s_{i} \neq s_{i}$, a contradiction. Thus, $\mathrm{C} 1$ is satisfied.

Next, let $\left(\omega^{\prime}, s^{\prime}\right) \in P_{i}^{\pi}(\omega, s)$ and $\left(\omega^{\prime \prime}, s^{\prime \prime}\right) \in P_{i}^{\pi}\left(\omega^{\prime}, s^{\prime}\right)$. Then, $s_{i}^{\prime \prime}=s_{i}^{\prime}=s_{i}$, so $\left(\omega^{\prime \prime}, s^{\prime \prime}\right) \in$ $P_{i}^{\pi}(\omega, s)$, and consequently, $P_{i}^{\pi}\left(\omega^{\prime}, s^{\prime}\right) \subseteq P_{i}^{\pi}(\omega, s)$. Since $s_{i}^{\prime}=s_{i}$, it holds that $(\omega, s) \in$ $P_{i}^{\pi}\left(\omega^{\prime}, s^{\prime}\right)$ as well, and the same arguments imply $P_{i}^{\pi}(\omega, s) \subseteq P_{i}^{\pi}\left(\omega^{\prime}, s^{\prime}\right)$. So, C2 is satisfied. 
Proof of Proposition 4.34. First note that since $\pi \in \Pi^{\mathrm{m}}$, for any $(\omega, s),\left(\omega^{\prime}, s^{\prime}\right) \in H^{\pi}$ with $s \neq s^{\prime}$, it holds that $\left(\omega, \lambda^{s}\right) \neq\left(\omega^{\prime}, \lambda^{s^{\prime}}\right)$. That is, no two distinct histories are mapped to the same posterior history. Thus, $\phi$ is a bijection.

Let $(\omega, s),\left(\omega^{\prime}, s^{\prime}\right) \in H^{\pi}$ and $i \in N$. If $(\omega, s) \in P_{i}^{\pi}\left(\omega^{\prime}, s^{\prime}\right)$, then $\phi(\omega, s)=\left(\omega, \lambda^{s}\right) \in$ $Q_{i}^{\pi}\left(\omega^{\prime}, s^{\prime}\right)$ by the definition of $Q_{i}^{\pi}\left(\omega^{\prime}, s^{\prime}\right)$. If $\left(\omega, \lambda^{s}\right)=\phi(\omega, s) \in Q_{i}^{\pi}\left(\omega^{\prime}, s^{\prime}\right)$, then $(\omega, s) \in$ $P_{i}^{\pi}\left(\omega^{\prime}, s^{\prime}\right)$. Therefore, $(\omega, s) \in P_{i}^{\pi}\left(\omega^{\prime}, s^{\prime}\right)$ if and only if $\phi(\omega, s) \in Q_{i}^{\pi}\left(\omega^{\prime}, s^{\prime}\right)$.

Suppose $Q_{i}^{\pi}(\omega, s) \cap Q_{i}^{\pi}\left(\omega^{\prime}, s^{\prime}\right) \neq \emptyset$. It follows that $P_{i}^{\pi}(\omega, s) \cap P_{i}^{\pi}\left(\omega^{\prime}, s^{\prime}\right) \neq \emptyset$, so $P_{i}^{\pi}(\omega, s)=$ $P_{i}^{\pi}\left(\omega^{\prime}, s^{\prime}\right)$. Therefore, $Q_{i}^{\pi}(\omega, s)=\phi\left(P_{i}^{\pi}(\omega, s)\right)=\phi\left(P_{i}^{\pi}\left(\omega^{\prime}, s^{\prime}\right)\right)=Q_{i}^{\pi}\left(\omega^{\prime}, s^{\prime}\right)$, so $\mathcal{Q}_{i}^{\pi}$ is a partition.

Proof of Lemma 4.37. Since $\pi \sim \pi^{\prime}$, for every $i \in N$ there is a bijection $\psi_{i}: S_{i}^{\pi} \rightarrow S_{i}^{\pi^{\prime}}$ such that, for every $\omega \in \Omega$, for every $s \in S^{\pi}, \pi^{\prime}(\psi(s) \mid \omega)=\pi(s \mid \omega)$.

Let $(\omega, s) \in H^{\pi}$ and $i \in N$.

We have that $\left(\omega^{\prime}, s^{\prime}\right) \in P_{i}^{\pi}(\omega, s)$ if and only if $\left(\omega^{\prime}, s^{\prime}\right) \in H^{\pi}$ and $s_{i}^{\prime}=s_{i}$ if and only if $\left(\omega^{\prime}, \psi\left(s^{\prime}\right)\right) \in H^{\pi^{\prime}}$ and $\psi_{i}\left(s_{i}^{\prime}\right)=\psi_{i}\left(s_{i}\right)$ if and only if $\left(\omega^{\prime}, \psi\left(s^{\prime}\right)\right) \in P_{i}^{\pi^{\prime}}(\omega, \psi(s))$.

Let $\left(\omega^{\prime}, \lambda^{\prime}\right) \in Q_{i}^{\pi^{\prime}}(\omega, \psi(s))$. Then, by the definition of $Q_{i}^{\pi^{\prime}}$, there is $\left(\omega^{\prime}, \psi\left(s^{\prime}\right)\right) \in P_{i}^{\pi^{\prime}}(\omega, \psi(s))$ with $\lambda^{\psi^{\left(s^{\prime}\right)}}=\lambda^{\prime}$. As shown in the previous paragraph, this implies $\left(\omega^{\prime}, s^{\prime}\right) \in P_{i}^{\pi}(\omega, s)$. Since by construction $\lambda^{s^{\prime}}=\lambda^{\prime^{\psi\left(s^{\prime}\right)}}=\lambda^{\prime}$, it follows that $\left(\omega^{\prime}, \lambda^{\prime}\right) \in Q^{\pi}(\omega, s)$ and therefore $Q_{i}^{\pi^{\prime}}(\omega, \psi(s)) \subseteq Q_{i}^{\pi}(\omega, s)$

Since $\sim$ is reflexive, we also have that $Q_{i}^{\pi}(\omega, s) \subseteq Q_{i}^{\pi^{\prime}}(\omega, \psi(s))$.

Proof of Lemma 4.39. Clearly, if $\mathcal{Q}_{i}^{\pi}=\mathcal{Q}_{i}^{\pi^{\prime}}$ then $\pi$ and $\pi^{\prime}$ are equally informative. For the other direction, assume that $\pi$ and $\pi^{\prime}$ are equally informative. As $\pi^{\prime}$ is as informative as $\pi$, for all $Q^{\prime} \in \mathcal{Q}_{i}^{\pi^{\prime}}$ there is $Q \in \mathcal{Q}_{i}^{\pi}$ such that $Q^{\prime} \subseteq Q$. As $Q^{\prime} \cap Q \neq \emptyset$ and as $\pi$ is as informative as $\pi^{\prime}$, it must hold that $Q \subseteq Q^{\prime}$, i.e., $Q^{\prime}=Q$. Thus, $\mathcal{Q}_{i}^{\pi^{\prime}} \subseteq \mathcal{Q}_{i}^{\pi}$. Using the same arguments one finds $\mathcal{Q}_{i}^{\pi} \subseteq \mathcal{Q}_{i}^{\pi^{\prime}}$.

Proof of Proposition 4.41. By Corollary 4.36 and Lemma 4.37 we can assume without loss of generality that $\pi \in \Pi^{\ell}$, so that $\mathcal{Q}_{i}^{\pi}=\mathcal{P}_{i}^{\pi}$ for all $i \in N$.

Let $i \in N$. Assume $Q \in \mathcal{Q}_{i}^{\pi}$ and $Q^{\prime} \in \mathcal{Q}_{i}^{\pi^{\prime}}$ are such that $Q \cap Q^{\prime} \neq \emptyset$. We first show Condition (ii) of Definition 4.38, i.e., $Q^{\prime} \subseteq Q$. Let $\left(\omega^{*}, \lambda^{*}\right) \in Q \cap Q^{\prime}$. There is $(\omega, \lambda) \in H^{\pi}$ such that $Q=Q_{i}^{\pi}(\omega, \lambda)=P_{i}^{\pi}(\omega, \lambda)$. Thus, by Lemma 4.29 , we have that $Q=P_{i}^{\pi}\left(\omega^{*}, \lambda^{*}\right)$. Consider $(\bar{\omega}, \bar{\lambda}) \in Q^{\prime}$. There is $\left(\omega^{\prime}, s^{\prime}\right) \in H^{\pi^{\prime}}$ such that $Q^{\prime}=Q_{i}^{\pi^{\prime}}\left(\omega^{\prime}, s^{\prime}\right)$ and there is $\left(\omega^{\prime \prime}, s^{\prime \prime}\right) \in P_{i}^{\pi^{\prime}}\left(\omega^{\prime}, s^{\prime}\right)$ with $\lambda^{s^{\prime \prime}}=\bar{\lambda}$. In particular, since $s_{i}^{\prime \prime}=s_{i}^{\prime}$, we have $\bar{\lambda}_{i}=\lambda_{i}^{\prime^{s^{\prime \prime}}}=\lambda_{i}^{s^{\prime}}=\lambda_{i}^{*}$. Since $\Lambda^{\pi^{\prime}} \subseteq \Lambda^{\pi}$, we have $(\bar{\omega}, \bar{\lambda}) \in \Lambda^{\pi}$, and since $\bar{\lambda}_{i}=\lambda_{i}^{*}$, we have $(\bar{\omega}, \bar{\lambda}) \in P_{i}^{\pi}\left(\omega^{*}, \lambda^{*}\right)=Q$. We have shown that $Q^{\prime} \subseteq Q$.

In order to prove Condition (i) of Definition 4.38 it is now sufficient to show that for each $Q^{\prime} \in \mathcal{Q}_{i}^{\pi^{\prime}}$ there is $Q \in \mathcal{Q}_{i}^{\pi}$ with $Q \cap Q^{\prime} \neq \emptyset$. Let $\left(\omega^{\prime}, s^{\prime}\right) \in H^{\pi^{\prime}}$ be such that $Q^{\prime}=Q_{i}^{\pi^{\prime}}\left(\omega^{\prime}, s^{\prime}\right)$. It holds that $\left(\omega^{\prime}, \lambda^{s^{\prime}}\right) \in Q^{\prime} \subseteq \Lambda^{\pi^{\prime}} \subseteq \Lambda^{\pi}$. Thus, there is $Q \in \mathcal{Q}_{i}^{\pi}$ with $\left(\omega^{\prime}, \lambda^{s^{\prime}}\right) \in Q$. 
Proof of Lemma 4.43. Observe that $(\omega, \lambda) \in \Lambda^{\pi}$ if and only if there is $s \in S^{\pi}$ such that $\lambda=\lambda^{s}$ and $\pi(s \mid \omega)>0$. This, however, is equivalent to $\pi^{\ell}(\lambda \mid \omega)=\sum_{s \in S^{\pi}: \lambda^{s}=\lambda} \pi(s \mid \omega)>0$, which holds if and only if $(\omega, \lambda) \in H^{\pi^{\ell}}=\Lambda^{\pi^{\ell}}$.

Proof of Proposition 4.46. First observe that for every $p \in \operatorname{relint}(P(\sigma))$ it holds that $p(\omega, \lambda)>0$ whenever there is $p^{\prime} \in P(\sigma)$ with $p^{\prime}(\omega, \lambda)>0$. Thus, for any such $p, p^{\prime}$ it holds that

$$
\Lambda^{\pi^{p^{\prime}}}=\left\{(\omega, \lambda) \in \Omega \times R(\sigma) \mid p^{\prime}(\omega, \lambda)>0\right\} \subseteq\{(\omega, \lambda) \in \Omega \times R(\sigma) \mid p(\omega, \lambda)>0\}=\Lambda^{\pi^{p}}
$$

So, by Corollary 4.42, it holds that $\pi\left(p^{\prime}\right)$ is at least as informative as $\pi(p)$.

Let $\pi^{\ell} \in \Pi^{\ell}$ be as defined in (4.6) and define $p^{\prime} \in P(\sigma)$ by

$$
p^{\prime}(\omega, \lambda)=\lambda^{0}(\omega) \pi^{\ell}(\lambda \mid \omega), \quad \omega \in \Omega, \lambda \in R(\sigma) .
$$

Then $\pi^{\ell}=\pi\left(p^{\prime}\right)$. Thus, as seen before, $\pi^{\ell}$ is at least as informative as $\pi^{p}$. Moreover, by Corollary 4.44, $\pi$ is at least as information as $\pi^{\ell}$. Hence, $\pi$ is at least as informative as $\pi^{p}$. 



\section{Bibliography}

Alonso, R. and O. Camara (2016). Bayesian persuasion with heterogeneous priors. Journal of Economic Theory 165, 672-706.

Alonso, R. and O. Câmara (2016). Persuading voters. American Economic Review 106(11), 3590-3605.

Arieli, I. and Y. Babichenko (2019). Private Bayesian persuasion. Journal of Economic Theory 182, 185-217.

Arieli, I., Y. Babichenko, F. Sandomirskiy, and O. Tamuz (2020). Feasible joint posterior beliefs.

Aumann, R. J. (1976). Agreeing to disagree. The annals of statistics, 1236-1239.

Aumann, R. J., M. B. Machler, and R. E. Stearns (Eds.) (1995). Repeated Games with Incomplete Information. MIT Press.

Austen-Smith, D. (1992). Explaining the vote: constituency constrains on sophisticated voting. American Journal of Political Science, 68-95.

Bala, V. and S. Goyal (2000). A noncooperative model of network formation. Econometrica $68(5), 1181-1229$.

Banks, J. S. and J. Duggan (2000). A bargaining model of collective choice. American Political Science Review 94(1), 73-88.

Bardhi, A. and Y. Guo (2018). Modes of persuasion toward unanimous consent. Theoretical Economics 13(3), 1111-1149.

Battaglini, M., R. B. Morton, and T. R. Palfrey (2010). The swing voter's curse in the laboratory. The Review of Economic Studies 77(1), 61-89.

Beauchêne, D., J. Li, and M. Li (2019). Ambiguous persuasion. Journal of Economic Theory $179,312-365$. 
Bergemann, D. and S. Morris (2016a). Bayes correlated equilibrium and the comparison of information structures in games. Theoretical Economics 11(2), 487-522.

Bergemann, D. and S. Morris (2016b). Information design, bayesian persuasion, and bayes correlated equilibrium. American Economic Review 106(5), 586-91.

Best, J. and D. Quigley (2016). Honestly dishonest: A solution to the commitment problem in bayesian persuasion. Technical report, Mimeo.

Bizzotto, J. and A. Vigier (2020). Can a better informed listener be easier to persuade? Economic Theory, 1-17.

Blackwell, D. (1953). Equivalent comparisons of experiments. The annals of mathematical statistics, 265-272.

Boleslavsky, R. and C. Cotton (2015). Grading standards and education quality. American Economic Journals: Microeconomics 7, 248-179.

Buechel, B. and L. Mechtenberg (2019). The swing voter's curse in social networks. Games and Economic Behavior 118, 241-268.

Bushman, R. M. and R. J. Indjejikian (1995). Voluntary disclosures and the trading behavior of corporate insiders. Journal of Accounting Research 33(2), 293-316.

Candogan, O. (2019). Persuasion in networks: Public signals and k-cores. Working paper.

Candogan, O. and K. Drakopoulos (2020). Optimal signaling of content accuracy: Engagement vs. misinformation. Operations Research 68(2), 497-515.

Chan, J., S. Gupta, F. Li, and Y. Wang (2019). Pivotal persuasion. Journal of Economic Theory 180, 178-202.

Colla, P. and A. Mele (2010). Information linkages and correlated trading. The Review of Financial Studies 23(1), 203-246.

Crawford, V. P. and J. Sobel (1982). Strategic information transmission. Econometrica: Journal of the Econometric Society, 1431-1451.

Degan, A. and A. Merlo (2007). Do voters vote sincerely? Technical report, National Bureau of Economic Research.

Denzau, A., W. Riker, and K. Shepsle (1985). Farquharson and fenno: Sophisticated voting and home style. American Political Science Review 79(4), 1117-1134.

Egorov, G. and K. Sonin (2019). Persuasion on networks. Working paper. 
Feddersen, T. J. and W. Pesendorfer (1996). The swing voter's curse. The American Economic Review 86, 408-424.

Felsenthal, D. S. and A. Brichta (1985). Sincere and strategic voters: An israeli study. Political Behavior 7(4), 311-324.

Fréchette, G. R., A. Lizzeri, and J. Perego (2019). Rules and commitment in communication: An experimental analysis. Technical report, National Bureau of Economic Research.

Galperti, S. and J. Perego (2020). Information systems. Working Paper.

Ganuza, J.-J. and J. S. Penalva (2010). Signal orderings based on dispersion and the supply of private information in auctions. Econometrica 78(3), 1007-1030.

Gentzkow, M. and E. Kamenica (2016). Competition in persuasion. The Review of Economic Studies 84(1), 300-322.

Groseclose, T. and J. Milyo (2010). Sincere versus sophisticated voting in congress: theory and evidence. The Journal of Politics 72(1), 60-73.

Grosser, J. and M. Seebauer (2016). The curse of uninformed voting: An experimental study. Games and Economic Behavior 97, 205-226.

Hedlund, J. (2017). Bayesian persuasion by a privately informed sender. Journal of Economic Theory 167, 229-268.

Heese, C. and S. Lauermann (2017). Robust voter persuasion. Working Paper.

Herings, P. J. J., D. Karos, and T. Kerman (2020). Belief inducibility and informativeness. Working Paper Available at SSRN: https://ssrn.com/abstract=3722145 or http://dx.doi.org/10.2139/ssrn.3722145.

Hintikka, J. (1962). Knowledge and belief: an introduction to the logic of the two notions.

$\mathrm{Hu}$, J. and X. Weng (2020). Robust persuasion of a privately informed receiver. Economic Theory, 1-45.

Ichihashi, S. (2019). Limiting sender's information in bayesian persuasion. Games and Economic Behavior 117, 276-288.

Inostrozosa, N. and A. Pavan (2020). Persuasion in global games with application to stress testing. Working Paper.

Jackson, M. O. and B. W. Rogers (2007). Meeting strangers and friends of friends: How random are social networks? The American Economic Review 97(3), 890-915. 
Jiménez-Martínez, A. (2015). A model of belief influence in large social networks. Economic Theory 59(1), 21-59.

Kamenica, E. and M. Gentzkow (2011). Bayesian persuasion. American Economic Review 101, 2590-2615.

Kerman, T., P. J. J. Herings, and D. Karos (2020). Persuading sincere voters. Working Paper available at SSRN: https://ssrn.com/abstract=3550071 or http://dx.doi.org/10.2139/ssrn.3550071.

Kerman, T. and A. P. Tenev (2021). Persuading communicating voters. Working Paper available at SSRN:.

Kleiner, A. and B. Moldovanu (2017). Content-based agendas and qualified majorities in sequential voting. American Economic Review 107(6), 1477-1506.

Kolotilin, A., T. Mylovanov, A. Zapechelnyuk, and M. Li (2017). Persuasion of a privately informed receiver. Econometrica 85(6), 1949-1964.

Kosenko, A. (2018). Bayesian persuasion with private information. Working Paper.

Kosterina, S. (2018). Persuasion with unknown beliefs. Work. Pap., Princeton Univ., Princeton, NJ.

Levy, G. (2004). A model of political parties. Journal of Economic theory 115(2), 250-277.

Li, C. (2017). A model of bayesian persuasion with transfers. Economics Letters 161, 93-95.

Lipnowski, E., D. Ravid, and D. Shishkin (2019). Persuasion via weak institutions. Available at SSRN 3168103.

Mathevet, L., J. Perego, and I. Taneva (2020). On information design in games. Journal of Political Economy 128(4), 1370-1404.

Milgrom, P. and N. Stokey (1982). Information, trade and common knowledge. Journal of economic theory 26(1), 17-27.

Min, D. (2017). Bayesian persuasion under partial commitment. Work. Pap., Univ. Ariz., Tucson.

Osborne, M. J. and A. Rubinstein (1994). A course in game theory. MIT press.

Osborne, M. J. and A. Slivinski (1996). A model of political competition with citizencandidates. The Quarterly Journal of Economics 111(1), 65-96. 
Poole, K. T. and H. Rosenthal (2000). Congress: A political-economic history of roll call voting. Oxford University Press on Demand.

Rick, A. (2013). The benefits of miscommunication.

Schnakenberg, K. E. (2015). Expert advice to a voting body. Journal of Economic Theory 160, $102-113$.

Taneva, I. (2019). Information design. American Economic Journal: Microeconomics 11(4), $151-85$.

Tsakas, E. and N. Tsakas (2017). Noisy persuasion. Working Paper.

Tsakas, E., N. Tsakas, and D. Xefteris (2021). Resisting persuasion. Economic Theory, 1-20.

Wang, Y. (2013). Bayesian persuasion with multiple receivers. Available at SSRN 2625399.

Ziegler, G. (2019). Adversarial bilateral information design. Technical report, mimeo, Northwestern University. 


\section{General Discussion}

Bayesian persuasion provides us with a framework to study social interactions in which one (or more) party is trying to persuade possibly multiple parties to take a certain action. The research in this area is divided into two main branches; the first one is interested in extending the basic model of Kamenica and Gentzkow (2011) in different ways, and the second one focuses on analyzing real world institutions and possibly improving them. This dissertation touches both branches by extending the basic model by considering multiple receivers, and by applying Bayesian persuasion in a voting context.

For the most part, we are interested in an information designer whose concern is not to improve social welfare, but to implement her desired outcome regardless of what the true state of the world might be. In other words, we focus on the sender's perspective, rather than the receivers'. Examples for such senders and situations can be a government that wishes to pass a bill by holding a referendum, a political party that wants to pass a motion in the U.S. Senate, or a board member of a non-profit organization that offers to take some action by putting a statement to an internal vote. This, however, does not necessarily mean that in this context social welfare is never improved; in many cases it is possible that the sender's preferred outcome actually provides a welfare improvement. Nevertheless, our concern is to determine optimal communication protocols for the sender that maximize the probability of her preferred outcome being implemented.

In Chapter 2, our focus lies on private communication, which means we focus on a sender who sends custom-tailored messages to the voters. It should be noted that we are considering a limit case: the sender is able to reach all voters and communicate with them privately. Although this is perhaps possible up to some degree via social media, with the current technology, implementing private communication in a way such that it covers all voters would be difficult.

We assume that voters vote sincerely, that is, they only take their own belief into account when they vote. Taking sincere voting as given, however, our initial findings tell us that if the sender is communicating in an optimal way, then sincere voting is not in any voter's best interest. In particular, we observe the swing voter's curse, which represents a situation where it is optimal for a receiver to vote against his belief. Thus, sincere voting does not constitute 
an equilibrium.

The more conventional approach would be to assume that voters vote strategically and to determine the Bayesian Nash equilibrium (BNE) strategies. We consider a different approach and instead of finding optimal strategies that arise in a BNE, we employ sincere voting as the equilibrium refinement. That is, we characterize an optimal communication protocol under which sincere voting $i s$ the optimal action for a voter. In other words, strategic voting coincides with sincere voting in equilibrium. This equilibrium selection, no doubt, would make Eugene V. Debs (whose quote I provided in the beginning of Chapter 2) very happy. ${ }^{5}$ According to Debs, one should vote for what she believes in, even if not doing so would provide a better outcome; we can interpret this as him being against strategic voting. While I do not share this idea with Debs, I am inclined to think that we would perhaps have a more "truthful" version of democracy if everyone voted only according to their true preferences.

In Chapter 3, we address an assumption that is frequently made and is often subject to criticism in Bayesian persuasion models: receivers do not communicate among themselves prior to taking their action. This is also the case in our model in CHAPTER 2; the voters only observe their own private message from the sender and update their beliefs. This criticism is right on the spot, since in reality people almost always communicate with a friend, family member, or colleague regarding an important decision. This is, of course, also the case for voting situations; it is common to deliberate with people you know before voting. Thus, incorporating this exchange of information between the voters seems like a very natural extension to such models. Technically, we assume that the voters are in a fixed and commonly known network and that they can see their neighbors' private messages.

To focus on the possible effects of different types of networks, we simply assume that each agent observes these additional messages from his neighbors with probability 1 and without a cost. A more realistic choice of modelling would be to make this decision of observing additional messages endogenous, or assuming that there is some cost (time, money) for acquiring this information. The introduction of the network, however, complicates the problem majorly as we cannot simplify the problem as we do in standard Bayesian persuasion models. Because of this reason, we try to keep the model as simple as possible to ensure some tractability.

It does not come as a surprise that the best situation for the sender is to face voters who do not communicate at all. In this case, the sender's power to manipulate is the greatest as voters do not share the obtained information. It is, however, surprising that the sender's capabilities do not decrease monotonically as the network gets denser. In other words, given two nonempty networks, it is possible that the sender would implement her preferred outcome with a higher probability in the denser network. While this seems counterintuitive at first glance, it

\footnotetext{
${ }^{5}$ Eugene V. Debs was a well known American socialist and political activist who lived in the late 19th and early 20 th century.
} 
is possible that a sender, knowing that the voters have access to more information, would be able to exploit these connections. The technical reason for this is that while the sender can replicate the information voters possess in any given network in the empty network, it might not be possible to replicate this information in a less dense network that is not empty.

In CHAPter 4, we deal with another important problem in information design: determining which beliefs of voters the sender can achieve. In other words, we do not consider a voting application; we are not interested in a sender that wishes to implement some outcome, but in beliefs of voters that are inducible (or feasible) for the sender. The importance of inducible beliefs stems from the fact that in Bayesian persuasion problems, the sender's belief almost always depends only on the beliefs of the receivers. We first show that there is a limit to the extent the sender can polarize the beliefs in a given population (with homogeneous beliefs). If the sender is communicating with two receivers in order to create disagreement among them, it is not possible to make one receiver believe a state is the true state of the world with probability 1, while making the other receiver believe this with probability 0. Given that we start with receivers that have the same prior beliefs, it is an intuitive result as in reality there is often a limit to the disagreement one can create among people.

Determining inducible distributions of beliefs, however, is a more complicated problem as it requires pinning down all the distributions that the sender can achieve. This characterization, in the single-receiver case, is very simple and can be done with only one condition: Bayes plausibility. Extending the model to multiple receivers, this simple characterization becomes insufficient since the sender is communicating possibly correlated signals to the receivers. The characterization we provide for inducible distributions of beliefs cover a more general case than in CHAPTER 2 or CHAPTER 3, since we assume there is an arbitrary finite state space as opposed to a binary one.

The notion of informativeness we introduce in this chapter differs from the informativeness notion considered in Bayesian persuasion models, which is according to Blackwell order (i.e. according to first-order beliefs). We, on the other hand, are interested in the informativeness of communication protocols that induce the same distribution of beliefs, but differ on the higher order information provided to the receivers. The motivation for focusing on such communication protocols is that given an inducible distribution of beliefs (which the sender wants to implement), we want to find the least informative way of implementing such beliefs, in the sense that receivers know as little as possible about each others' beliefs of the true state. This is particularly important for a sender who wishes to create disagreement among people. If one receiver knows that another receiver actually knows what the true state is, then there wouldn't be a disagreement between them as the choice for action would be left to the receiver who knows the truth.

Thus, we first classify communication protocols as minimal, direct, and language independent, depending on the types of messages they employ, and compare their informativeness 
according to this new definition. We provide a characterization for a least informative communication protocol that induces a given distribution and finally show that it lies in the relative interior of the set of all language independent communication strategies which induce that distribution. If a communication protocol is in the relative interior of this set, it follows that all signals realize with positive probability in all states. Thus, a receiver deems all states possible, so it is harder for a receiver to infer what another receiver knows about the true state. 


\section{Summary}

\section{Chapter 2 - Bayesian Persuasion in Elections with Sincere Voting}

In this chapter, we focus on targeted messages in elections. We employ a Bayesian persuasion framework to analyze a situation, where a sender (e.g. an incumbent party) wishes to pass a proposal and is communicating with multiple (and identical) receivers (e.g. an electorate) prior to the vote. While the sender only wants to implement the proposal, each receiver wants the proposal to pass only if it is "good" for society.

We observe that if the sender communicates publicly, then she can increase the probability of implementing the proposal drastically. However, we find that there is a better way for the sender to communicate, which is by allowing for private and correlated messages. Under sincere voting, private communication improves upon public communication considerably. But it is not without a caveat: it is not in the voters' best interest to vote sincerely under the optimal communication strategy. More precisely, the optimal communication strategy leads to the swing voter's curse, that is, receivers are better off voting against their beliefs. Hence, sincere voting is not an equilibrium.

As assuming sincere voting makes the model tractable and since it has also empirically been shown to be not uncommon, we employ sincere voting as the equilibrium selection. In particular, we characterize an optimal communication strategy that leads to a Bayesian Nash equilibrium (BNE) under sincere voting. We find that while this optimal communication strategy also improves upon public communication, it is not without cost to the sender to implement sincere voting as a BNE: the probability of implementing her preferred outcome is lower than the case where voters are not strategic. 


\section{Chapter 3 - Bayesian Persuasion in Communication Networks}

The motivation for this chapter stems from the fact that in Bayesian persuasion problems with multiple receivers, it is often assumed that the receivers do not communicate with each other prior to taking their action. However, in reality, people usually deliberate before voting or ask the opinion of a friend simply before buying a product. We incorporate this exchange of information between receivers in a Bayesian persuasion framework by assuming that (identical) receivers are connected by an exogenously given and commonly known network. The sender wants to implement a proposal and commits to a communication strategy that sends private and correlated messages to the receivers. On top of their own private realizations, receivers can also observe messages of their neighbors in the network, and after updating their beliefs, vote sincerely on the proposal. We examine how networks of shared information affect the sender's gain from persuasion.

We first show that introducing a network structure (such that receivers also observe their neighbors' messages) leads to the failure of several assumptions that can be made without loss of generality in standard Bayesian persuasion models (e.g. anonymity, straighforwardness, truth-telling in the sender's preferred state of the world). This complicates the problem of finding an optimal communication strategy for any given network. However, we are still able to determine sufficient conditions for optimality given some specific types of networks.

In exploring optimality, we start by showing that the sender's gain from persuasion is highest under the empty network. There are, however, non-empty networks under which the sender can do as good as under the empty network. We provide such sufficiency conditions for also the star, circle, and line networks, together with some general results on regular and irregular networks. Finally, perhaps surprisingly, we show that it is possible for the sender to fare better under a denser network. One would expect that the sender's gain from persuasion is monotonically decreasing with the number of links in a network, however, there exist situations where the sender can exploit the connections between receivers and achieve a higher probability of implementing her preferred outcome. 


\section{CHAPTER 4 - Belief Inducibility and Informativeness}

The subjects of this chapter are inducible distributions of posterior beliefs and the informativeness of different classes of communication strategies that induce a distribution. Since the sender's payoff is often only determined by the beliefs of receivers, determining feasible distributions of posterior beliefs is an important problem in information design. When there is a single receiver, the only restriction on the inducibility of a distribution of beliefs is Bayes plausibility, that is, the mean of the posteriors is equal to the prior. When we extend the model to multiple receivers, however, Bayes plausibility is no longer sufficient for inducibility. Hence, we first characterize, under a finite state space, the additional conditions required to ensure that a distribution is feasible for the sender. Further, we define three classes of communication strategies; minimal, direct, and language independent, and show that any distribution of posterior beliefs can be induced by a direct, and also by a language independent communication strategy (LICS).

Given that a distribution is inducible, there are often multiple communication strategies that induce it. While obviously all such communication strategies induce the same first order beliefs, they may differ in the higher order information they provide. In situations where the sender wishes to restrict this higher order information receivers have about each others' information about the state, she faces the problem of choosing a communication strategy that provides the least amount of such information.

For this purpose, we introduce a new definition for informativeness of communication strategies that induce a distribution. That is, we define informativeness according to the higher order information communication strategies induce. In particular, one communication strategy is more informative than the other if, after each agent receives a message, the set of the pairs of states and other agents' messages (or posteriors) he deems possible is smaller under the former than the latter. We then show that the "least informative" communication strategy belongs to the class of LICS's, and provide a sufficient condition for an LICS to be least informative. 


\section{Impact}

"Always pass on what you have learned."

- Yoda, Star Wars: Return of the Jedi

One of the last things Yoda advised Luke to do before passing away was to teach others, new Jedi apprentices, what he had learned about the force. Luke, at that point in the story, was the last remaining Jedi. So, it was crucial for the survival of the Jedi Order that Luke passed on what he had learned from Yoda and from his own research of the force. Fans of Star Wars would agree that passing these teachings on to the next generation had an immense impact on the Star Wars universe (most would argue that it is a positive one). In our ordinary, mundane universe, unfortunately, passing on new findings from one's research might not have such a drastic effect. Nevertheless, I would argue that social sciences are cumulative and that even small contributions help paving the way to a better understanding of human behavior.

In the broadest of terms, the research in this dissertation can be classified as microeconomics. To be more specific, it is a game theoretical analysis of voting situations in an information design (Bayesian persuasion) framework. Persuasion can be defined as the act of providing information to someone in order to influence their behavior (via influencing their beliefs). In Bayesian persuasion, agents react to the information they receive in a Bayesian (or in other words, in a rational) way. The research provided in this dissertation mainly focuses on $(i)$ the theoretical analysis of targeted communication in elections, where a sender (e.g. an incumbent party) is trying to persuade receivers (e.g. an electorate) to vote in favor of a proposal (that is either "good" or "bad" for the society), and (ii) the analysis of the sender's ability to manipulate receivers' beliefs and the informativeness of communication protocols that lead to those beliefs.

The literature on information design bas been booming since Kamenica and Gentzkow (2011) introduced Bayesian persuasion, and it has two main branches of research. While the first one is interested in extending the standard Bayesian persuasion model (single sender, single receiver) and focuses on the designer's optimization problem, the other branch deals with applications of Bayesian persuasion into different contexts (e.g. financing, insurance, medical research). The dissertation aims to contribute to both strands of research; to the 
former by extending the standard model to multiple receivers and focusing on the sender's problem under private communication, and to the latter by considering a voting application.

When Yoda was passing on what he knew about the force to Luke, he was actually passing on a cumulative knowledge that many other Jedis that came before him contributed to. Similar to Yoda adding to this cumulative knowledge of the force, this dissertation contributes to an immense body of work on Bayesian persuasion, and (hopefully) contributes to painting a clearer picture in voting applications.

In problems that involve collective decision making, there are two main ways to model voter behavior: $(i)$ sincere voting or $($ ii $)$ strategic voting. While under sincere voting an agent takes into account only his own belief when choosing his action, under strategic voting, an agent also considers the actions of other agents. In CHAPTER 2, we assume that voters vote sincerely; however, we observe that under the optimal communication structure, sincere voting is not in any voters best interest. This phenomenon is known as the swing voter's curse in the voting literature and refers to situations where a voter is better off voting against their belief. In our model, this stems from the fact that upon observing their message, agents are pivotal only in one state of the world (when the proposal is "bad" for them).

The contribution of CHAPTER 2 primarily lies in employing sincere voting as the equilibrium selection, as opposed to finding Bayes Nash equilibrium strategies under strategic voting. In other words, we wish to circumvent the swing voter's curse and want sincere voting to be the optimal action for a voter. We find that ensuring sincere voting is an equilibrium depends crucially on receivers not being pivotal (in any state). Implementing sincere voting as an equilibrium is not without a cost though; the sender has a lower probability of implementing the proposal.

In most voting applications of Bayesian persuasion, it is often assumed that voters do not communicate with each other prior to taking their action. In reality, however, we know that people often deliberate before making a final decision, which could be on voting for a political candidate or simply on buying a product. CHAPter 3 builds on CHAPTER 2 and incorporates communicating voters in attempting to contribute to filling this gap in the literature. There are many modelling choices when incorporating exchange of information between receivers. We assume that voters are in an exogenously given and commonly known network, and that they can observe their neighbors' private messages. This can be thought of as the sender communicating with the receivers via an online platform and receivers being able to observe messages of their "friends" on the platform.

This seemingly simple modification, however, complicates the problem of finding the optimal way to communicate for the sender greatly: many simplifications that we can make without loss of generality under the standard Bayesian persuasion model fail. Still, even in the absence of these simplifications, we are able to provide optimal communication structures for some specific types of networks. Perhaps the most surprising result in ChaPter 3 
(maybe not for experts on networks) is a counterintuitive one: it is possible for the sender to fare better in a network with more connections, or in other words, in a denser network. Initially, one is inclined to think that if there are more possibilities for communication in the network, then this does not bode well for the sender as her messages, in a sense, become less private. However, it turns out that while the sender prefers the empty network to any other, given two non-empty networks, it is possible that the sender implements the proposal with a higher probability under the denser one. That is, there exist situations in which the sender can manipulate the receivers more when there are more links in the network.

In information design, but also in many other sender-receiver models, the sender's payoff is often determined only by the receiver's belief about the true state of the world. This makes determining what is "feasible" in terms of beliefs for the sender an important task in Bayesian persuasion problems. In CHAPTER 4, we refrain from considering a voting application; in particular, we do not assume any particular underlying game and focus on the problem of inducible (feasible) distributions of beliefs. When there is a single receiver, inducible beliefs can be characterized by one simple condition, Bayes plausibility, which simply means that the mean of the posterior beliefs is equal to the prior belief. This follows easily in the single receiver case, as the receiver is updating his belief via Bayes rule. But when the model is extended to multiple receivers, Bayes plausibility alone is not sufficient for inducibility. In contrast to Chapter 2 and Chapter 3 in which we assume there are only two states of the world, we allow for an arbitrary finite state space and we provide a characterization of inducible distributions of beliefs by identifying the additional conditions required for a distribution to be inducible.

While Chapter 4 is on information design, it also contributes to the literature on knowledge. In particular, we define a new notion of informativeness for communication protocols, which depends on the higher order information of receivers. In the Bayesian persuasion literature, informativeness is considered in terms of Blackwell (1953), that is, according to first-order beliefs. We, on the other hand, focus on communication structures that induce the same first-order beliefs and distinguish their informativeness by the information they have about other receivers' information about the true state. We then employ this definition to provide a characterization of the set of least informative communication protocols.

This definition comes in handy when the sender wishes to induce a particular distribution of beliefs but also wishes to restrict the information receivers have about each others' beliefs. If you are someone who wants to polarize a group, you may wish to restrict what one subgroup knows about the other subgroup's knowledge of the state, as it would increase the probability of disagreement within the group. When Emperor Palpatine (a.k.a. Darth Sidious) plants the seeds of betrayal into Anakin Skywalker's mind, he always restricts some information about what the Jedi Order knows about the force, which consequently leads to the ultimate polarization between Anakin and the Jedi Order when Anakin becomes (spoiler alert) Darth 
Vader.

The results in this dissertation will probably be interesting for other academics who are interested in information design, but I also hope it will be an interesting topic for a more general audience who are interested in other branches of game theory as well. It attempts to fill in some gaps in the literature and help paint a more complete picture, and my hope is that it will be useful for researchers that will work in this area in the future. All chapters in the dissertation are submitted to various journals as individual papers and are currently under review. I have presented these works in numerous conferences so far, and I hope that it will reach more researchers in the future after being published.

As much as theorists like to talk about the scientific impact of their research, for some mysterious reason, they generally seem to be uncomfortable when discussing its immediate social impact. While I do not see a problem with "art for art's sake" (I do not in any way mean this dissertation is a work of art), I also do not agree that there aren't any social implications of even a very abstract, theoretical study. In the case of this dissertation, we can infer from Chapter 2 that it is costly for the sender to ensure sincere voting and further, the sender should target a slightly larger portion of the electorate than is required to pass a proposal if she wishes to achieve this. Incorporating exchange of information between receivers, CHAPTER 3 tells us that more communication may not always be bad for the sender. Given some types of connections between receivers, the sender may even not be affected at all (relative to the empty-network) by communicating voters. CHAPTER 4, on the other hand, tells us that there is a limit to the degree of polarization the sender can achieve between the receivers and the higher order information of receivers plays an important role in achieving this.

In any case, the immediate social impact of this dissertation will not be as powerful as the social impact of an empirical case study. Nevertheless, something that seems relatively less useful at one time might very well prove to be effective in another.

"Difficult to see. Always in motion is the future."

- Yoda, Star Wars: The Empire Strikes Back 


\section{Biography}

Toygar T. Kerman was born on 3rd of April 1987 in Fairfax/Virginia, USA. In 2009, he received his B.Sc. diploma in Mathematics from Bilkent University in Turkey. He then obtained an M.Sc. in Actuarial Sciences from Middle East Technical University (M.E.T.U.) in 2012. After completing the courses and while writing his thesis at M.E.T.U., he started his masters in Actuarial Sciences and Mathematical Finance at the University of Amsterdam in 2011, and

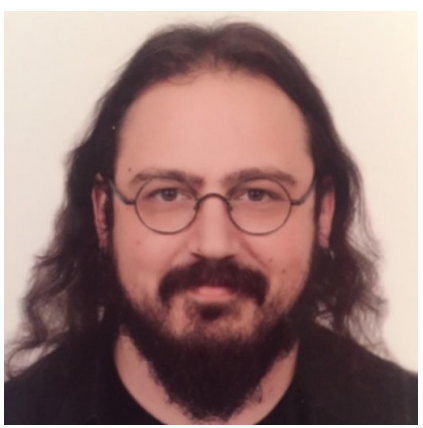
obtained an M.Sc. in 2013. He then returned to Turkey and started a Ph.D. in Economics at Bilkent University in 2014. After spending 3 years at Bilkent and on track to completing his Ph.D., he decided to leave Bilkent and obtained an M.A. in Economics from Bilkent University in 2017.

He was a Ph.D. candidate in the Department of Microeconomics and Public Economics at Maastricht University between 2017 and 2021, under the supervision of P. Jean-Jacques Herings and Dominik Karos. The results of his research at Maastricht University are collected in this dissertation. He presented his work in several international conferences such as the Stony Brook International Conference on Game Theory and the European Meeting on Game Theory (SING). His paper Persuading Communicating Voters (joint work with Anastas P. Tenev) received the best paper award at the GamesNet Young Scholars' Competition, which is the subject of CHAPTER 3 of this dissertation. 